\title{
REVISITING THE CHOLINERGIC HYPOTHESIS IN THE DEVELOPMENT OF ALZHEIMER'S DISEASE
}

\author{
LAURA A. CRAIG \\ Bachelor of Science, University of Calgary, 2001 \\ Master of Science, McGill University, 2004
}

A Thesis

$\therefore$ Submitted to the School of Graduate Studies

of the University of Lethbridge

in Partial Fulfillment of the

Requirements for the Degree

DOCTOR OF PHILOSOPHY

\author{
Department of Neuroscience \\ University of Lethbridge \\ LETHBRIDGE, ALBERTA, CANADA
}

(C) Laura A. Craig, 2008 


\begin{abstract}
Alzheimer's disease $(\mathrm{AD})$ is the most common form of dementia affecting the elderly population today; however, there is currently no accurate description of the etiology of this devastating disorder. No single factor theory has been demonstrated as being causative; however, an alternative theory suggests that the interaction of multiple risk factors is responsible for $\mathrm{AD}$. In this thesis I present data suggesting a neuroprotective role for acetylcholine during aging. Using a rat model of cholinergic depletion of the medial septum, I explored the effects of four common risk factors for $\mathrm{AD}$ (stress, seizures, stroke and circadian dysfunction) targeted at the hippocampus and examined the effects on measures of hippocampal dependent (water maze) and hippocampal independent (fear conditioning) memory. Here, I propose a role for acetylcholinemediated compensatory mechanisms in the functional recovery observed following subthreshold insults similar to those commonly observed in the elderly.
\end{abstract}




\section{Acknowledgements}

First and foremost I would like to thank my supervisor, Dr. Robert McDonald. Thank you for your support and encouragement, and for allowing me the independence to pursue my own projects and to learn from my experiences. I would also like to thank Nhung Hong for doing the majority of the cholinergic depletion and stroke surgeries used in this thesis and for teaching me the techniques. I would like to give a huge thank you to Joelle Kopp who was an invaluable asset who assisted in histology and spent many hours on the microscope counting cholinergic neurons. I would also like to thank Dr. Christine Werk for patiently reading and re-reading early drafts of my papers and this thesis and Dr. Hugo Lehmann for all his help in running statistics and interpreting my data. In addition, many thanks go out to Courtney Lamb, Robert Court and Arthur Verhoef for helping me with histology and behavioural testing, to Keri Colwell for running the corticosterone assays, the Metz lab for kindly allowing me the use of their microscope and to Karen Dow-Cazel and the rest of the animal care staff for taking care of my rats and helping me set up the circadian experiements. 


\section{Table of Contents}

Title page

Page

Signature page

Abstract $\quad$ iii

Acknowledgements $\quad$ iv

Table of contents $\quad \mathrm{V}$

List of tables $\quad x$

List of figures $\quad$ xi

List of abbreviations $\quad$ xiv

Chapter 1: General introduction 1

What is Alzheimer's disease? 3

$\begin{array}{ll}\text { Models of Alzheimer's disease } & 6\end{array}$

The cholinergic hypothesis of Alzheimer's disease $\quad 6$

$\begin{array}{lr}\text { The basal forebrain cholinergic system } & 8\end{array}$

The role of the basal forebrain cholinergic system in learning and memory $\quad 10$

The current state of the cholinergic hypothesis of Alzhimer's disease 15

$\begin{array}{ll}\text { Risk factors for Alzheimer's disease } & 16\end{array}$

Multiple combinations of co-factors theory of Alzheimer's disease 22

The role of the hippocampus in learning and memory 24

$\begin{array}{ll}\text { Objective of the present thesis } & 25\end{array}$

Chapter 2: A series of pilot studies to determine the threshold for behaviourally subthreshold stroke, seizures and stress

Abstract 
Experiment 1a: The effect of a chronic variable stress schedule on performance of the water maze task

Materials and Methods

Results

Experiment 1b: The effects of a variable restraint stress procedure on levels of blood borne CORT

Materials and Methods

Results

Discussion

Experiment 2: The effects of various non-convulsive doses of kainic acid on water maze performance

Materials and Methods

Results

Discussion

Experiment 3: The effects of a focal sub-threshold dose of Endothelin-1 on water maze performance

Materials and Methods

Results

Discussion

Conclusion

Chapter 3: Chronic circadian disruption impairs hippocampal memory in the rat

Abstract

Introduction

Materials and Methods 
Discussion

Conclusion

Chapter 4: Reduced cholinergic status in hippocampus produces spatial memory deficits when combined with kainic acid induced seizures

Abstract

Introduction

Materials and Methods

Results

Discussion

Conclusion

Chapter 5: Selective lesion of medial septal cholinergic neurons followed by a mini-stroke impairs spatial learning in rats

Abstract

Introduction

Materials and Methods

Results

Discussion

Conclusion

Chapter 6: Emergence of spatial impairment in rats foltowing specific cholinergic depletion of the medial septum combined with chronic stress

Abstract

Introduction

Experiment 1: The effects of cholinergic depletion and chronic stress on levels of blood borne CORT 
Experiment 2: The effects of cholinergic depletion and chronic stress on the performance of a hippocampal dependent task

Materials and Methods

Results

Experiment 3: The effects of cholinergic depletion and chronic stress on the performance of a hippocampal independent task

Results

Discussion

Conclusion

Chapter 7: Circadian disruption in the cholinergic depleted rat does not affect circadian rhythmicity or learning and memory

Abstract

Introduction

Experiment 1: The effects of cholinergic depletion of the medial septum on measures of circadian rhythmicity and their response to phase shifting

Materials and Methods

Results

Experiment 2: The effects of cholinergic depletion of the medial septum followed by phase shifting on measures of learning and memory

Materials and Methods

Results

Discussion 
Conclusion

Chapter 8: General discussion

Sub-threshold models of risk factors for Alzheimer's disease

Cholinergic depletion combined with an active risk factor

Cholinergic depletion combined with a passive risk factor

The cholinergic hypothesis of Alzheimer's disease

A neuroprotective or compensatory role for acetylcholine in response to injury

A look at my results using the 'new' cholinergic hypothesis

Multiple combinations of co-factors model of Alzheimer's disease

Treatment implications

Future studies

Conclusions 


\section{List of Tables}

Page

Table 1.1 Risk factors for Alzheimer's disease $\quad 17$

Table 1.2 Passive and active risk factors for Alzheimer's disease $\quad 24$

Table 3.1 Light/Dark schedule used to induce acute and chronic phase shifting $\quad 70$

Table 4.1 Cholinergic depletion combined with kainic acid: ChAT positive cell counts

Table 5.1 Cholinergic depletion combined with stroke: ChAT positive cell counts

Table 6.1 Cholinergic depletion combined with stress: ChAT positive cell counts

Table 7.1 Cholinergic depletion combined with phase shifting: Light/Dark schedule used to induce phase shifting

Table 7.2 Cholinergic depletion combined with phase shifting: Measures of circadian rhythmicity

Table 7.3 Cholinergic depletion combined with phase shifting: ChAT positive cell counts

Table 8.1 Summary of results 


\section{List of Figures}

Figure 1.1 Synthesis of acetylcholine

Figure 2.1 Platform location for Rapid Acquisition Task

Figure 2.2 Chronic stress- Water maze performance

Figure 2.3 Chronic stress- Corticosterone levels

Figure 2.4 Sub-convulsive seizures- Water maze performance

Figure 2.5 Sub-convulsive seizures- Seizure severity

Figure 2.6 Sub-threshold stroke- Water maze performance

Figure 3.1 Circadian disruption- Representative actograms

Figure 3.2 Circadian disruption- Measures of circadian rhythmicity

Figure 3.3 Circadian disruption- Water maze performance

Figure 3.4 Circadian disruption- Platform preference

Figure 3.5 Circadian disruption- Performance on the fear conditioning tasks

Figure 4.1 Cholinergic depletion combined with kainic acid: Seizure severity

Figure 4.2 Cholinergic depletion combined with kainic acid: Water maze performance

Figure 4.3 Cholinergic depletion combined with kainic acid: Platform preference

Figure 4.4 Cholinergic depletion combined with kainic acid: Visible platform training

Figure 4.5 Cholinergic depletion combined with kainic acid: Coronal sections of medial septum labeled with $\alpha$-ChAT and $\alpha$-parvalbumin, and hippocampus stained with $\mathrm{AChE}$

Figure 4.6 Cholinergic depletion combined with kainic acid: Extent of the cholinergic lesion

Figure 4.7 Cholinergic depletion combined with kainic acid: Coronal section of cresyl violet stained hippocampus 
Figure 5.1 Cholinergic depletion combined with stroke- Water maze performance

Figure 5.2 Cholinergic depletion combined with stroke- Visible platform training

Figure 5.3 Cholinergic depletion combined with stroke- Coronal sections of medial septum labeled with $\alpha$-ChAT and $\alpha$-parvalbumin, and hippocampus stained with AChE

Figure 5.4 Cholinergic depletion combined with stroke- Extent of the cholinergic lesion

Figure 5.5 Cholinergic depletion combined with stroke- Volumetric analysis of hippocampal damage

Figure 5.6 Cholinergic depletion combined with stroke- Coronal section of cresyl violet stained hippocampus

Figure 6.1 Cholinergic depletion combined with stress- Corticosterone levels

Figure 6.2 Cholinergic depletion combined with stress- Water maze performance

Figure 6.3 Cholinergic depletion combined with stress- Platform preference

Figure 6.4 Cholinergic depletion combined with stress- Aided placements

Figure 6.5 Cholinergic depletion combined with stress- Performance on the fear conditioning tasks

Figure 6.6 Cholinergic depletion combined with stress- Coronal sections of medial septum labeled with $\alpha$-ChAT and $\alpha$-parvalbumin, and hippocampus stained with $\mathrm{AChE}$

Figure 6.7 Cholinergic depletion combined with stress- Extent of the cholinergic lesion

Figure 6.8 Cholinergic depletion combined with stress- Coronal section of cresyl violet stained hippocampus

Figure 7.1 Cholinergic depletion combined with phase shifting- Representative actograms

Figure 7.2 Cholinergic depletion combined with phase shifting- Water maze performance

Figure 7.3 Cholinergic depletion combined with phase shifting- Performance on the 
Figure 7.4 Cholinergic depletion combined with phase shifting- Coronal sections of medial septum labeled with $\alpha$-ChAT and $\alpha$-parvalbumin, and hippocampus stained with $\mathrm{AChE}$

Figure 7.5 Cholinergic depletion combined with phase shifting- Extent of the cholinergic lesion

Figure 7.6 Cholinergic depletion combined with phase shifting- Coronal section of cresyl violet stained hippocampus 


\section{List of Abbreviations}

AMPA- $\alpha$-amino-3-hydroxy-5-methyl-4-isoxazole propionic acid

$A \beta$ - amyloid beta fragment

$A \beta_{40^{-}}$amyloid beta fragment ( $40 \mathrm{bp}$ )

$\mathrm{A} \beta_{42}$ - amyloid beta fragment (42 bp)

ACh- acetylcholine

AChE- acetylcholinesterase

AChEI- acetylcholinesterase inhibitor

AD- Alzheimer's disease

ANOVA- analysis of variance

APOE $\varepsilon 4-$ apolipoprotein E $\varepsilon 4$

APP- amyloid precursor protein

$\beta$-APP- soluable beta amyloid fragment

BACE1/2- Beta-site APP cleaving enzyme $1 / 2$

BDNF- brain derived neurotrophic factor

CBF- complete basal forebrain

ChAT- choline acetyltransferase

CORT- corticosterone

CT- computerized tomography

DAB- diaminobenzidine

EDTA- ethylenediaminetetraacetic acid

EGF- epidermal growth factor

ET-1- Endothelin-1

FGF- fibroblast growth factor

FTDP-17- fronto-temporal dementia with Parkinsonism linked to chromosome 17

GABA- $\gamma$-aminobutyric acid

HDB- horizontal limb of the diagnol band of Broca

IgG-SAP- 192 IgG Saporin

IP- interpeduncular nucleus

LD- light/dark

LTP- long term potentiation

MRI- magnetic resonance imaging

MS/VDM- medial septum and vertical limb of the diagonal band of Broca

NGF- nerve growth factor

NMDA- N-methyl-D-aspartate

OB- olfactory bulbs

P75 NTR- $\mathrm{p} 75$ neurotrophin receptor

PBS- phosphate buffered saline

PFA- paraformaldehyde

PFC- prefrontal cortex

PS1/2- presinilins 1 and 2

IgG-SAP- 192 IgG Saporin

LTP- long term potentiation

$\mathrm{mAChR}$ - muscarinic acetylcholine receptor

MS- medial septum 
MS/VDM- medial septum and vertical limb of the diagonal band of Broca nAChR- nicotinic acetylcholine receptor

PBS- phosphate buffered saline

PFA- paraformaldehyde

SCN-suprachiasmatic nucleus

VIP- vasoactive intestinal peptide

VP- vasopressin 


\section{Chapter 1}

General Introduction 
Alzheimer's disease (AD) is a devastating neurodegenerative disorder that currently affects approximately 300,000 individuals in Canada $(\sim 7 \%$ of the population over 65) and according to the Alzheimer's Society of Canada, this number is expected to increase to 750,000 by 2031 . The financial cost of caring for these patients, who often spend 3-4 years in an institution, was estimated at $\$ 3.9$ billion annually in 1994, and is thought to be closer to $\$ 5.5$ billion today (Ostbye and Crosse, 1994; Alzheimer's Society of Canada). Apart from the financial costs, the emotional cost to caregivers, family and friends of the patient is immeasurable. Patients suffering from $\mathrm{AD}$ exhibit a severe loss of memory, coupled with dramatic mood swings, personality changes and sleep disorders that influence all aspects of their daily living.

Memory loss is the most noticeable characteristic of $\mathrm{AD}$. The inital impairment reported by $A D$ patients and/or their caregivers can be described as mild forgetfulness for recent experiences, but eventually patients begin to experience difficulty recollecting more distant memories (Carlesimo and Oscar-Berman, 1992; Fleischman and Gabrieli, 1999). As AD progresses, patients' memory becomes severely affected leading to an incapacitating memory loss (Carlesimo and Oscar-Berman, 1992). This severe, progressive loss of memory often leads to a diagnosis of "Alzheimer's-like" dementia by the $6^{\text {th }}$ or $7^{\text {th }}$ decade of life and patients are given a life expectancy of 8-12 years.

A diagnosis of $\mathrm{AD}$ can only be made conclusively by a post-mortem autopsy that reveals the presence of senile plaques and neurofibrillary tangles (the characteristic neuropathology of $\mathrm{AD}$ ). Unfortunately, there are currently no treatments that are able to completely stop or slow the progression of this disorder, though some patients do obtain temporary relief of symptoms from approved drugs targeting the cholinergic or 
glutamatergic neurotransmitter systems. One of the major deterrents to progress in this field is a lack of understanding as to what precisely causes $\mathrm{AD}$. There have been a number of single factor theories proposed to explain the etiology of $\mathrm{AD}$, but to date, no one theory can adequately explain all aspects of this disorder. Determining the cause of a neurodengerative disorder such as $\mathrm{AD}$ is essential to the development of effective treatments and the discovery of an eventual cure for these patients.

\section{What is Alzheimer's disease?}

$\mathrm{AD}$ is a neurodegenerative disorder that is characterized by a severe and progressive loss of memory, combined with characteristic neuropathology such as amyloid plaques, neurofibrillary tangles and alterations in neurotransmitter levels, the most prominent of which is a loss of acetylcholine (ACh) in the cortex and hippocampus (Mesulam, 2004). This disorder was first presented as a single case study of a woman who suffered from early onset dementia with "unusual neuropathology" in 1907 by Alois Alzheimer, but it was not until 1911 when more patients had been described that the disorder became know as "Alzheimer's disease" (Alzheimer, 1907, 1911). The original definition of $\mathrm{AD}$ was one of early onset senile dementia combined with neurofibrillary tangles and neuritic plaques. It is important to note that while tangles were first described by Alzheimer, both dementia and the occurrence of plaques in the aged brain had been previously described (as reviewed in Goedert et al., 2006). The note-worthy aspect of these cases was their early onset in middle age. Since the original description of $A D$, this definition has changed to encompass both presenile ( $<65$ years of age at onset) and senile ( $>65$ years of age at onset) forms of dementia. Presenile dementia is characterized by its early onset, rapid progression, and its hereditary nature, while senile dementia occurs 
later in life with a slower progression and has no known cause (although numerous risk factors have been associated with this form).

Classically, the first symptom of $\mathrm{AD}$ is memory loss; specifically, impairments in epsiodic memory (memory for personal events and experiences) are the first to be reported by $\mathrm{AD}$ patients, and/or their caregivers (Carlesimo and Oscar-Berman, 1992). This deficit initially can be described as forgetfulness for recent experiences, but eventually patients begin to experience difficulty recollecting more distant memories (Carlesimo and Oscar-Berman, 1992; Tulving and Markowitsch, 1998). Semantic memory (knowledge of objects, facts and concepts) is also negatively affected at early stages of $\mathrm{AD}$ (Hodges and Patterson, 1994). The processing of both episodic and semantic memory is dependent on medial temporal lobe structures that begin to show signs of damage in the early stages of $\mathrm{AD}$, suggesting a correlation between structure and function in the progression of dementia (Hodges and Patterson, 1994). Other forms of memory such as implicit memory (procedural or habit learning) remain relatively intact until the latest stages of $\mathrm{AD}$ (Elridge et al., 2002; Fleischman et al., 2005).

Senile plaques are one of the two defining features of $\mathrm{AD}$ and were noted by Alzheimer in his original description (Alzheimer, 1907). These plaques have since been characterized as being made up of deposits of the protein amyloid (Glenner et al., 1984; Masters et al., 1985). The amyloid precursor protein (APP) is normally (nonamyloidogenic pathway) cleaved by $\alpha$-secretase to produce a soluble non-toxic amyloid protein, and by $\gamma$-secretase to produce two non-amyloidogenic fragments. A second (amyloidogenic) pathway produces the soluble $\beta$-amyloid protein ( $\beta$-APPs), when $\beta$ secretase cleaves APP at an alternative site, and one of two plaque forming $\beta$-amyloid 
fragments $\left(A \beta_{40}\right.$ or $\left.A \beta_{42}\right)$ following cleavage by $\gamma$-secretase. Both $A \beta$ fragments and $\beta$ APPs are thought to be neurotoxic as reported in both in vitro and in vivo studies (Lue et al., 1999; Verdile et al., 2004), but others have found no toxicity associated with either $\mathrm{A} \beta$ or $\beta$-APPs (Robinson and Bishop, 2002; Verdile et al., 2004). The $\mathrm{A} \beta_{40 / 42}$ fragments are deposited as diffuse plaques, which aggregate into mature plaques in the cortex and spread from there into subcortical areas. While amyloid plaques are a characteristic sign of $\mathrm{AD}$, it is important to note that these plaques are often seen in the brains of cognitively intact individuals. Moreover, a correlation has been reported between the number of plaques and the severity of dementia in $\mathrm{AD}$ patients (Naslund et al., 2000), but this relationship is not as strong as that seen with neurofibrillary tangles (Arragada et al., 1992; Guillozet et al., 2003; Barnes et al., 2006).

The second defining neuropathology of $\mathrm{AD}$ is the presence of neurofibrillary tangles which are made up of the protein tau (a cytoskeletal protein that normally binds to microtubules and regulates polymerization in healthy neurons; Goedert et al., 1988). Tau proteins become pathological when they undergo hyperphosphorylation causing it to selfaggregate and form tangles. These tangles interfere with axonal transport leading to atrophy of axons and dendrites and the eventual death of the neuron such as that observed in $\mathrm{AD}$ brains (Goedert et al., 2006). While specific point mutations in the gene coding for tau have been identified and are thought to cause the abnormal phosphorylation, this process is not fully understood. Tangles originate in subcortical areas (predominantly the hippocampus) and spread out to cortical areas and show a high correlation with cognitive deficits in AD (Arragada et al., 1992; Tiraboschi et al., 2004). 


\section{Models of Alzheimer's disease}

Severe progressive memory loss accompanied by plaques and tangles found in the brain at autopsy are essential to a diagnosis of $\mathrm{AD}$. Any animal model proposed to study this disorder should be able to reproduce at least one (and preferably more) of these characteristic changes in cognitive ability and/or neuropathology. The most common models for $\mathrm{AD}$ research use transgenic mice that have been developed to overexpress mutated human genes implicated in AD (e.g., APP, PS1/2, APOE ع4, FTDP-17). As they age, these mice develop plaques and/or tangles, cognitive impairment and reductions in cholinergic activity (Morley, 2002; Oddo et al., 2003; Oddo et al., 2003b; Belluci et al., 2006). Studies in transgenic mice have resulted in an enormous amount of information describing the role of genetic factors to the development of $A D$. Other models of $A D$ explore the effects of non-genetic risk factors for $\mathrm{AD}$ (e.g., stress, ischemia, excitotoxicity), on measures of learning and memory and/or changes in APP or tau neuropathology (Nabeshima and Nitta, 1994; Panegyres, 1998; Bennett et al., 2000; Han et al., 2008).

\section{The cholinergic hypothesis of Alzheimer's disease}

The cholinergic hypothesis was the first theory proposed to explain $\mathrm{AD}$ and has since led to the development of the only drugs currently approved to treat mild to moderate $\mathrm{AD}$ (Bartus et al., 1982; Bartus, 2000). This theory was based on the finding that a loss of cholinergic activity in the brains of $\mathrm{AD}$ patients (Davies and Maloney, 1976; Perry et al., 1981) coupled with experimental studies in humans and non-human primates. These studies reported that by blocking central cholinergic activity with scopolamine young subjects would demonstrate memory deficits similar to those seen in 
aged individuals. These impairments could be reversed by treatment with the cholinergic agonist physostigmine (Drachman and Leavitt, 1974; Drachman, 1977; Bartus, 1978). This theory led to early clinical studies utilizing another type of cholinergic agonist, acetylcholinesterase inhibitors (AChEIs) that initially showed promise in reversing the memory impairment in $\mathrm{AD}$ patients.

There are currently three AChEIs (donepezil, rivastigmine and galantamine) that have been approved by the Food and Drug Administration for the treatment of mild to moderate $\mathrm{AD}$ in Canada (Alzheimer's Society of Canada). All of these drugs have been reported to have similar effectiveness; however, donepezil is the most widely prescribed. Clinical trials have reported small improvements in cognition and global functioning by treating patients with donepezil compared to placebos, but these effects were not permanent and patients still demonstrated a decline in cognitive functioning over time (Doody et al., 2001). Courtney et al. (2004) have reported similar small improvements on measures of cognition and functionality, but after two years, there were no differences in the overall progress of disability or rate of institutionalization in donepezil treated patients compared to placebo-treated controls. Taken together these studies show that AChEIs can provide small amounts of symptomatic improvement but no long term cure for this disorder. Moreover, not all $\mathrm{AD}$ patients are responsive to the effects of AChEIs and to date the differences between 'responders' and 'non-responders' remains unclear (Connelly et al., 2005; Lemstra et al., 2007). Connelly et al. (2005) have reported that $\mathrm{AD}$ patients with high levels of medial temporal lobe atrophy are less likely to respond to donepezil and other studies have suggested that patients carrying the APOE $\varepsilon 4$ allele are 
more likely to benefit from donepezil than non- APOE $\varepsilon 4$ carriers (Bizzarro et al., 2005; Choi et al., 2008).

The failure of $\mathrm{AChEIs}$ to cure $\mathrm{AD}$ was seen by some researchers as disproving the cholinergic hypothesis and the focus of $\mathrm{AD}$ research shifted away from acetylcholine (ACh) and towards $A \beta$ and mutations in APP processing genes as more likely causative factors. However, others have continued to explore the role of this neurotransmitter in the development of $\mathrm{AD}$ in hopes of explaining the consistency of this depletion in the brains of AD patients (Bartus, 2000; Mesulam, 2004).

\section{The basal forebrain cholinergic system}

The basal forebrain is a subcortical structure made up of a heterogeneous population of neurons that is important for many aspects of cognitive function. Of this neuronal population (made up of cholinergic, $\gamma$-aminobutyric acid (GABA)-ergic, and peptidergic neurons) the cholinergic neurons have been extensively studied, due to their involvement in $\mathrm{AD}$. Cholinergic neurons release the excitatory neurotransmitter $\mathrm{ACh}$ that is important for alertness and attention and can also play a role in both cortical and hippocampal plasticity. These neurons have high affinity choline transporters that internalize choline which is essential for the production of ACh (Karczmar et al., 2007). Figure 1.1 shows the complete reaction for ACh that is catalyzed by choline acetyltransferase (ChAT; a commonly used marker for cholinergic neurons) and broken down by acetylcholinesterase (AChE; commonly used as a measure of cholinergic activity) 


\section{$\mathrm{ChAT} \rightarrow$ \\ Acetyl Coenzyme A + Choline $\rightarrow$ ACh + Coenzyme A \\ $\leftarrow \mathrm{AChE}$}

Figure 1.1: Reaction for the synthesis of acetylcholine

There are two types of cholinergic receptors: nicotinic receptors (nAChR) and muscarinic receptors (mAChR). The mAChRs are seven transmembrane spanning Gprotein metabotropic receptors. Apart from ACh, these receptors can also be activated by carbochol and pilocarpine, and antagonized by scopolamine and atropine. All of the $\mathrm{mAChR}$ subtypes can be found distributed throughout the brain, especially in the cortex and hippocampus (Karczmar et al., 2007). The nAChRs are ligand gated ion channels that can be activated by nicotine, and antagonized by mecamylamine. These receptors are also distributed throughout the cortex and hippocampus (Karczmar et al., 2007).

The cholinergic system has widespread projections throughout the brain. The basal forebrain of both human and non-human animals can be divided into four distinct regions based on connectivity and localization. Progressing in a rostral-caudal direction, the first region is the medial septum (MS) which projects through the fimbria-fornix to the hippocampus. The second region is the vertical limb of the diagonal band of Broca (VDB) which also projects to the hippocampus, but has additional projections to the cingulate cortex and hypothalamus. The third region is the horizontal limb of the diagonal band of Broca (HDB) that projects to the olfactory bulbs and entorhinal cortex. The final and largest region of the basal forebrain is the nucleus basalis (NB) that projects to the neocortex, amygdala and hypothalamus (Woolf and Butcher, 1982; Mesulam et al., 1983). Although not the focus of this thesis, it is worthwhile to note two additional 
cholinergic projections that arise from the pedunculopontine nucleus of the pontomesencephalic reticular formation and from the laterodorsal tegmental gray of the periventricular area of the brain stem that provide the majority of cholinergic input to the thalamus and hypothalamus, with some indirect connections to the cortex (Mesulam et al., 1983). This pattern of connectivity is remarkably similar in rats, monkeys and humans (Mesulam et al., 1983) making the rat an ideal subject to study the function of the cholinergic system in an animal model.

\section{The role of the basal forebrain cholinergic system in learning and memory}

The role of the basal forebrain cholinergic system has been extensively explored through a number of different methodologies. The first studies used centrally acting cholinergic agonists and antagonists in humans and non-human animals to enhance and impair learning and memory respectively. These studies were followed by animal studies using excitotoxins to damage cholinergic nuclei in the basal forebrain. The most recent studies have used a specific immunotoxin to damage cholinergic neurons of the basal forebrain and this technique has resulted in the most valid data to date describing the role of ACh in learning and memory.

\section{Drug studies}

Cholinergic agonists and antagonists have been long recognized as being capable of modulating learning and memory. Early studies in young adult human and non-human primates found that treatment with scopolamine could impair memory to levels comparable to aged subjects. It was then shown that cholinergic agonists such as nicotine and pilocarpine, as well as physostigmine (an $\mathrm{AChEI}$ ) not only were able to promote learning and memory and improve performance on their own, but could reverse the 
scopolamine induced deficit suggesting an important role for the cholinergic system in learning and memory (Drachman, 1977; Bartus, 1978). Studies in rats have found a similar pattern of results as scopolamine, or other cholinergic antagonists can impair both spatial and non-spatial learning, and memory, and these impairments can be minimized or reversed by treatment with cholinergic agonists (reviewed in McGaughy et al., 2000).

\section{Excitotoxic lesions}

A second method for determining the role of cholinergic projections in learning memory involves irreversibly damaging the basal forebrain neurons. These initial experiments utilized mechanical or electrolytic lesions to disconnect the basal forebrain neurons from the hippocampus, amygdala and cortical areas; however, this method damaged both axons of passage and non-cholinergic neurons, which did not allow for an accurate interpretation of the specific role of cholinergic neurons in memory (Schliebs, 1998; McGaughy et al., 2000). The next evolution in lesion technique used excitotoxins that were able to damage cell bodies while leaving projections intact by binding to glutamate receptors on the cell surface and through an over release of $\mathrm{Ca}^{2+}$ destroyed the cell. By using the excitotoxin ibotenic acid to damage the basal forebrain, many independent groups were able to mimic the previously observed scopolamine related deficits on a wide variety of tasks. Apart from the behavioural impairment, ibotenic lesions reduced cortical and hippocampal ACh levels by 30-50\% (Murrey and Fibiger, 1985; Dunnett et al., 1987; Ridley et al., 1988; Page et al., 1991; Waite and Thal, 1996). These findings played a significant role in providing support for the cholinergic hypothesis of $\mathrm{AD}$ and the ibotenic lesion of the basal forebrain was proposed as a useful animal model for $\mathrm{AD}$. 
The ibotenic acid model was short lived due to the discovery of two novel excitotoxins: $\alpha$-amino-3-hydroxy-5-methyl-4-isoxazole propionic acid (AMPA) and quisqualic acid. The use of these drugs to damage the basal forebrain produced a significantly higher cholinergic depletion and less non-specific damage to surrounding areas. Surprisingly, this also resulted in a milder behavioural impairment than expected and no effect at all on some tasks. These findings contributed to a re-examination of the role of ACh in learning and memory. Although the overall cognitive deficits were not as global as previously reported (using ibotenic acid), animals with AMPA- or quisqualateinduced lesions were impaired on the 5-choice serial attention task (Muir et al., 1994), passive avoidance (Dunnett et al., 1987; Page et al., 1991) and conditional discrimination (Marston et al., 1994). Some of these deficits could be reversed by treatment with cholinergic agonists (Murrey and Fibiger, 1985; Ridely et al., 1988) or by transplanted grafts from basal forebrain cholinergic neurons (Muir et al., 1994). Taken together these results still pointed to a role for ACh in learning and memory, but it was not as essential a role as once thought.

192-IgG Saporin lesions

While these more specific and thorough lesions were beginning to clarify the role of ACh in learning and memory, there were still a number of problems with the model. The discovery of the specific immunotoxin 192 IgG Săporin (IgG SAP) was met with great enthusiasm as this toxin was able to damage cholinergic neurons with minimal to no non-specific damage (Wiley et al., 1991). The $192 \mathrm{IgG}$ antibody is raised against the p75 neurotrophin receptor ( $\mathrm{p} 75 \mathrm{NTR}$ ) and conjugated to the ribosome-inactivating protein saporin to form the immunotoxin IgG SAP. When IgG SAP binds to the p75 NTR it is 
internalized where the saporin inhibits protein synthesis and destroys the cell (Wiley et al., 1991). The p75 NTR is found on basal forebrain cholinergic neurons of the MS, $\mathrm{VDB}, \mathrm{HDB}$ and $\mathrm{NB}$ making this a useful tool to explore the role of $\mathrm{ACh}$ in learning and memory. Using this immunotoxin, it has since been demonstrated that collective damage to both the cholinergic and the non-cholinergic neurons of the basal forebrain were responsible for the impairments observed following excitotoxic lesions (Pang et al., 2001).

The original experiments that utilized IgG SAP infused the drug into the lateral ventricles and found impairment in spatial memory (Berger-Sweeney et al., 1994; Waite et al., 1995, 1999). This method produces a reliable lesion of basal forebrain neurons, but also damages Purkinje cells in the cerebellum that express the p75 NTR. Motor impairment due to this non-specific damage is thought to account for the spatial memory deficit in these early studies (Waite et al., 1995, 1999; Parent and Baxter, 2004). More recent studies have infused IgG SAP directly into the MS/VDB or the NB and have not reported impaired learning and memory on spatial learning tasks. Several groups have reported that discrete lesions of either the MS/VDB or the NB, as well as a complete lesion of the entire basal forebrain (CBF) did not impair spatial working memory as tested in standard versions of the water maze and radial arm maze tasks (Baxter et al., 1995; Pizzo et al., 2002; Vuckovich et al., 2004; Frielingsdorf et al., 2006). However, other groups using more challenging, or more sensitive tasks of spatial memory have reported deficits following MS/VDB lesions (Baxter et al., 1995; Bannon et al., 1996; Chang and Gold, 2004), NB lesions (Baxter et al., 1995) or CBF lesions (Pizzo et al., 2002; Frick et al., 2004). A prediction arising from these studies was that the cholinergic 
system may play a role in strategy selection, rather than spatial memory per se. Janis et al. (1998) reported a preference for a cue-based strategy following CBF lesions in the cue-place version of the water maze task. However, this study was carried out 48 hours post surgery at a time when the cholinergic lesion is not complete. More recent studies have found an increased preference for a spatial strategy in rats with MS/VDB lesions (Bizon et al., 2003; Jonasson et al., 2004).

The effects of IgG SAP lesions on non-spatial memory tasks have not been as extensively studied, but a few studies have reported no effect on freezing behaviour during fear conditioning to tone or to context following a selective MS/VDB lesion (Craig et al., 2008) or a complete CBF lesion (Frick et al., 2004). Attentional processes are also affected following a NB lesion as tested on the 5-choice serial reaction task (Lehmann et al., 2003; Harati et al., 2008). While MS/VDB lesions do not affect 5choice serial reaction task performance (Lehmann et al., 2003), this lesion does impair latent inhibition, which is a specific form of attentional processing (Baxter et al., 1997). Berger-Sweeney et al. (2000) has also found impaired social transmission of food preference in rats with either a MS/VDB or a NB lesion. Overall, it appears that cholinergic projections to the hippocampus and cortex do not play a major role in spatial learning and memory as previously suggested by early drug and excitotoxin studies. The most consistent findings following cholinergic depletion have been impaired attention tested directly using the 5-choice serial reaction task, or indirectly when using more challenging behavioural tasks which may also have an attentional or decision-making component. 


\section{The current state of the cholinergic hypothesis of Alzheimer's disease}

The results of the IgG SAP lesion studies, combined with unsuccessful clinical trials have clearly demonstrated that a loss of $\mathrm{ACh}$ is not the sole cause of $\mathrm{AD}$. However, the consistency of this finding in $\mathrm{AD}$ patients (Davies and Maloney, 1976; Perry et al., 1981; Rossor et al., 1984; Quirion, 1993; Mesulam, 2004) suggests that, while not causal, the cholinergic depletion plays an important role in the development of $\mathrm{AD}$. One such role may be involved in directly contributing to $\mathrm{AD}$ neuropathology.

Clinical studies have reported a positive correlation between the extent of the cholinergic depletion with the number of diffuse plaques in the non-demented elderly (Beach et al., 1997) and with the number of tangles in the brains of AD patients (Arendt et al., 1999). In vitro studies carried out in cultured cells, or in cortical and hippocampal slices have demonstrated a role for mAChRs in the processing of APP. Treatment with muscarinic agonists promoted the non-amyloidogenic pathway and increased the secretion of soluble APP while decreasing the production of the plaque forming A $\beta$. This effect could be blocked with muscarinic antagonists (Nitsch et al., 1992; Pittel et al., 1996; Qiu et al., 2003). Moreover, studies in vivo have shown that cholinergic depletion of the basal forebrain can increase overall levels of APP in the hippocampus and cortex of rats (Leanza, 1998; Lin et al., 1999; Aztiria et al., 2008). This increase can be reversed in part by transplanting cholinergic cell grafts (Aztiria,et al., 2008) or by treatment with a muscarinic agonist (Lin et al., 1999). The interaction between the cholinergic system and the development of neurofibrillary tangles is not as well described. In vitro studies have found muscarinic agonists are able to decrease tau phosphorylation, while nicotinic agonists tend to increase tau phosphorylation (Kar et al., 2002). Additional studies are 
required to accurately describe the direct effects of ACh on tau phosphorylation.

Alternatively, cholinergic depletion may have an indirect effect on tau phosphorylation as increased $\mathrm{A} \beta$ has been found to increase tau and the development of tangles (Rubio et al., 2006). A second possibility that will be explored further in this thesis is that ACh plays a protective role in the brain, and in its absence, individuals are more susceptible to additional risk factors associated with $\mathrm{AD}$.

\section{Risk factors for Alzheimer's disease}

The most prominent and best studied risk factors for $\mathrm{AD}$ are amyloid plaques, neurofibrillary tangles and the cholinergic depletion, but there are many other factors that have been correlated with $\mathrm{AD}$, especially the senile form of the disorder. Several single

factor theories of causation have been proposed to explain $\mathrm{AD}$ (Bartus et al., 1982; Hardy and Allsop, 1991; Arriagada et al., 1992; Munoz and Feldman, 2000; de la Torre, 2002), but it is most likely that a combination of multiple risk factors act synergistically to produces different variations of this disorder (Mesulam, 1999; McDonald, 2002; Driscoll et al., 2007; Craig et al., 2008; Kivipelto et al., 2008; McDonald et al., 2008). The risk factors that have been proposed as causing or putting an individual at risk for $\mathrm{AD}$ can be loosely divided into three categories: genetic, environmental and physical (Table 1.1). Most of these risk factors have not be proposed as causal factors, but correlative evidence, and their effects on learning and memory and $\mathrm{AD}$ neuropathology suggest that they may play a role in the development of this disorder. Some of the more common risk factors will be discussed in more detail. 
Table 1.1: A partial list of risk factors associated with the development of Alzheimer's disease

\begin{tabular}{l|l|l}
\hline \multicolumn{1}{c|}{ Genetic } & \multicolumn{1}{|c}{ Environmental } & \multicolumn{1}{c}{ Physical } \\
\hline APP & Circadian disruption & Stroke \\
PS1 & Stress & Seizures \\
PS2 & Aluminum & Loss of neurotransmitters \\
APOE $\varepsilon 4$ & Environmental toxins & Apoptosis \\
BACE1 & Prions & Glucose metabolism \\
BACE2 & Dietary factors & Head Trauma \\
FTDP-17 & Estrogen & Senile plaques \\
& Low education level & Neurofibrillary tangles \\
& & Oxidative stress \\
& & High blood pressure \\
& & Vascular disease \\
\hline
\end{tabular}

\section{Genetic risk factors}

Genetic risk factors include mutations in genes, or changes in allele distribution that are most often associated with the presenile form of $\mathrm{AD}$. There are a number of well characterized mutations found in genes involved in the processing and expression of APP and $\mathrm{A} \beta$ that have been shown to cause $\mathrm{AD}$ (Selkoe and Podlisny, 2002). The most common mutations arise in the presenilin 1 gene (PS1) that increases production of the more toxic $A \beta_{42}$ fragment either through alterations in $\gamma$-secretase activity, or possibly, by shifting the binding site on APP to promote cleavage of $A \beta_{42}$. Mutations in the APP gene itself are rare, but these mutations also enhance levels of $A \beta_{42}$ leading to increased plaque formation (Verdile et al., 2007). These mutations have been used to develop transgenic mice that develop plaques and/or tangles as they age, accompanied by cognitive deficits 
and cholinergic depletion (reviewed in Games et al., 2006). One of these models, the $3 \times \mathrm{Tg}-\mathrm{AD}$ mouse developed by Oddo et al. $(2003,2003 \mathrm{~b})$ is promising as these mice express mutated APP, PS1 and tau genes simultaneously, and develop both plaques and tangles in a pattern similar to that seen in $\mathrm{AD}$ patients, making this an exciting new model to presenile familial $\mathrm{AD}$. While there have been no mutations in the FTDP-17 gene that codes for tau detected in $\mathrm{AD}$ patients to date, over 30 mutations in this gene have been identified and linked to other dementias that present with neurofibrillary tangles, suggesting that a similar mutation may still be found in $\mathrm{AD}$ patients (Goedert $e t$ al., 2006).

There is one genetic risk factor that has been associated with senile $\mathrm{AD}$ and that is the presence of the apolipoprotein E $\varepsilon 4$ (APOE $\varepsilon 4$ ) allele. Individuals with two copies of this allele are at a significantly greater risk of developing $\mathrm{AD}$ than those with a single copy, or none at all (Corder et al., 1993). Interestingly the presence of APOE $\varepsilon 4$ has been correlated with increased deposits of $A \beta_{40 / 42}$ regardless of whether the individual develops $\mathrm{AD}$ or not (Berr et al., 1994) suggesting that this is one of many risk factors that is not causal on its own, but may interact with other factors to cause this disorder. It is thought that the APOE $\varepsilon 4$ allele decreases the clearance of $A \beta_{40 / 42}$ and/or enhances the aggregation of $A \beta_{40 / 42}$ fragments into senile plaques (Selkoe and Podlisny, 2002). APOE $\varepsilon 4$ has also been associated with decreases in cholinergic activity and decreased plasticity suggesting two more ways that this allele could lead to $\mathrm{AD}$ (Arendt et al., 1997)

\section{Environmental risk factors}

Environmental risk factors include exposure to specific external toxins, dietary factors or a lifestyle that have been associated with the development of $\mathrm{AD}$. 


\section{Chronic stress}

Chronic stress has been shown to be a risk factor for $\mathrm{AD}$ and Wilson et al. (2006) found that individuals who were prone to stress had a higher risk of developing AD than individuals who were not prone to stress. Moreover, elevated levels of glucocorticoids [the stress hormone: cortisol in human and corticosterone (CORT) in rats] have been correlated with poor cognitive aging in rats and humans (Lupien et al., 1998; Montaron et al., 2006). In patients diagnosed with $\mathrm{AD}$, Davis et al. (1986) found increases in blood cortisol levels compared to non-AD controls. In addition, increased cortisol appears to be linked to hippocampal atrophy due to prolonged increases in glucocorticoid levels resulting in the over stimulation of their specific receptors, most of which are located in the hippocampus (Sapolsky, 1999).

Chronic stress and glucocorticoids have also been found to increase both plaques and tangles in transgenic mouse models of $\mathrm{AD}$ (Green et al., 2006; Jeong et al., 2006; Rissman et al., 2007). Green et al. (2006) reported naturally elevated levels of CORT in the $3 \times \mathrm{Tg}-\mathrm{AD}$ mouse. Following chronic treatment with dexamethasone (to further elevate CORT levels) they found that $A \beta$ and tangles were apparent at a much earlier age in these mice compared to untreated controls, suggesting an acceleration of the progression of this disorder as a result of stress (Green et al., 2006). Jeong et al. (2006) found a similar pattern of neuropathology combined with a deficit in passive avoidance learning following stress in a mouse model of $\mathrm{AD}$. Taken together, these studies provide support for a role for chronic stress in the development (or acceleration) of $\mathrm{AD}$ neuropathology. 


\section{Circadian disruption}

Circadian disruption is one of the most common complaints of $\mathrm{AD}$ patients and is the number one reason for institutionalization of $\mathrm{AD}$ patients (Pollack and Perlick, 1991; Hatfield et al., 2004). These disruptions appear in the form of increased night time awakenings and day time napping, as well as the characteristic "sundowning" behaviour (increased agitation and activity at dusk) and are accompanied by decreases in the hormone melatonin. These changes are apparent in both the pre-clinical and early clinical stages of $\mathrm{AD}$, making them potential early markers for this disorder (Wu et al., 2003). Interestingly, Wu et al. (2003) have also shown that treatment with melatonin reduced neuropathology and increased survival in a mouse model of $\mathrm{AD}$. In addition, there appears to be a stronger link between circadian dysfunction and the resultant cognitive impairment, than age alone. Hamsters with disordered circadian rhythms have greater difficulty learning a conditional place preference than age matched control hamsters with regular circadian rhythms (Antoniades et al., 1999). However, there have been no direct reports of phase shifting or circadian disruption causing the neuropathological signs of $\mathrm{AD}$.

Physical risk factors

Physical risk factors are factors that have been shown to directly damage the brain as the result of some trauma or event that leads to neuronal death (e.g., stroke) or due to changes in neurotransmitter levels (e.g., ACh).

\section{$\underline{\text { Seizure activity }}$}

Seizures and epilepsy become more common as an individual ages and seizure activity has been shown to result in cell death, particularly in the hippocampus, making it 
a potential risk factor for the development of $\mathrm{AD}$. The development of late onset seizures or epilepsy is a common occurrence in AD patients (Hauser et al., 1986; Romanelli et al., 1990; Hesdorffer et al., 1996) and some studies have even suggested that individuals suffering from epilepsy may be at risk of developing $\mathrm{AD}$ (Breteler et al., 1995;

Thompson and Duncan, 2005). Studies in both humans and animals have shown subtle cognitive deficits following seizures, particularly in tasks requiring hippocampal involvement (Cavazos et al., 1994; Gayoso et al., 1994; Breteler et al., 1995; Gilbert et al., 1996; Thompson and Duncan, 2005; Marques et al., 2007). Seizure induced and other forms of excitotoxicity (e.g., increases in reactive oxygen species) appear to be linked to neurodegenerative diseases (Reiter, 1995). A few studies have examined the effects of seizures on the development of AD neuropathology. Increases in specific APP transcripts are found following kainic acid induced seizures (Panegyres, 1998) and these transcripts have been found to be upregulated in $\mathrm{AD}$ brains (Johnston et al., 1988; Tanaka et al., 1992) suggesting a possible relationship between the two. Moreover, Mohajeri et al. (2002) found increased susceptibility to seizures and increased $A B$ in a transgenic mouse model of $\mathrm{AD}$ subjected to pilocarpine seizures. The effects of seizure activity on tangle formation have not been explored, but kainic acid treatment does causes tau hyperphosphorylation in neurons prior to undergoing apoptotic cell death (Crespo-Biel et al., 2006).

\section{$\underline{\text { Stroke }}$}

Individuals who have suffered a stroke or transient ischemic attack are at a significantly greater risk of developing $\mathrm{AD}$ than the rest of the population. Indeed, the most common form of dementia found in a community based study was 'mixed 
dementia' where patients showed signs of both vascular pathology, as well as the traditional plaques and tangles (Snowden et al., 1997; Schneider et al., 2007). This finding has revived interest in Alzheimer's (1907) original case study in which he noted vascular abnormalities in the brain of his patient and has led to a vascular origins theory of $\mathrm{AD}$ (de la Torre, 2002). Moreover, subcortical infarcts are commonly found in the brains of $\mathrm{AD}$ patients and their presence increases the severity of the dementia (Snowden et al., 1997; Song et al., 2007). It is interesting to note that there is significant overlap in the risk factors for vascular disease and for $A D$, as factors such as hypertension, high cholesterol, the APOE $\varepsilon 4$ allele and $A \beta$ deposits in blood vessels have been associated with both stroke and $\mathrm{AD}$ (de la Torre, 2002; Luchsinger et al., 2005). Recent studies have found $A \beta$ deposits and tau phosphorylation in the aged rat brain following either chronic hypoperfusion or stroke (Bennett et al., 2000; Han et al., 2008) and Sun et al. (2006) found increased levels of $\beta$-secretase and $A \beta$ following stroke in a transgenic mouse model of $\mathrm{AD}$. Taken together these studies provide strong evidence for stroke as a risk factor for $\mathrm{AD}$.

\section{Multiple combinations of co-factors theory of Alzheimer's disease}

As an alternative to single factor theories of $\mathrm{AD}$ that do not adequately account for both the clinical and neuropathological symptoms of AD, McDonald (2002) proposed a co-factors model of aging in an attempt to explain the-etiology of $\mathrm{AD}$. This model predicts that different combinations of multiple risk factors for $\mathrm{AD}$ interact to produce variants of this disorder. One of the strengths of this model is that it can explain the individual differences observed between $\mathrm{AD}$ patients, and the differential response to treatment of similarly demented individuals. The underlying theory of the model is that 
AD is fundamentally a hippocampal dementia (Ball et al., 1984; Hyman et al., 1984) and that the risk factors associated with $\mathrm{AD}$ all have converging effects on this structure leading to neuronal damage and death accompanied by progressive cognitive decline.

Risk factors for $\mathrm{AD}$ have previously been classified as genetic, environmental or physical, but McDonald (2002) has proposed an alternative classification that can narrow this to two groups; passive factors (stress, circadian disruption, cholinergic depletion, amyloid plaques), which can cause damage to, or increase the vulnerability of hippocampal neurons, and active factors (stroke, seizures, neurofibrillary tangles, head trauma) that have the ability to directly disrupt learning and memory, through the death or dysfunction of hippocampal neurons. These passive factors do not noticeably affect learning and memory, although if more passive factors are present prior to the occurrence of an active factor it should increase the severity of the dementia. It is important to note that many risk factors can be classified as either passive or active factors depending on their severity. For example, severe and prolonged stress can damage neurons and produce cognitive impairment (acting as an active factor), but mild to moderate stress has no obvious effect on either measure. Nonetheless, mild to moderate stress can increase the susceptibility of the hippocampus to subsequent damage (acting as a passive factor). Table 1.2 lists some of these risk factors and their usual classification; although depending on the severity, each of these factors has the potential to change from passive to active and vice versa. 
Table 1.2: Risk factors for $\mathrm{AD}$ can be classified as passive or active factors

\begin{tabular}{l|l}
\hline \multicolumn{1}{c|}{ Passive Factors } & \multicolumn{1}{c}{ Active Factors } \\
\hline Stress & Stroke \\
Cholinergic depletion & Seizures \\
Circadian disruption & Head trauma \\
Amyloid plaques & Neurofibrillary tangles \\
\hline
\end{tabular}

\section{The role of the hippocampus in learning and memory}

The classification of $\mathrm{AD}$ as a hippocampal dementia is supported by the important role of the hippocampus in memory, and the awareness that the earliest forms of memory lost in $\mathrm{AD}$ are hippocampal dependent. The importance of the hippocampus for memory was most dramatically illustrated by the now famous case of H.M. and other patients that underwent bilateral medial temporal lobe resection in an attempt to treat epilepsy or schizophrenia (Scoville and Milner, 1957). These patients suffered retrograde amnesia for recent events and exhibited severe antereograde amnesia. This inability to form new memories and difficulty remembering past events suggested a role for the hippocampus in encoding new memories, storage and retrieval. Since then, several studies in rats and humans have confirmed the importance of the hippocampus in memory, particularly in the areas of spatial navigation, episodic memory, and memory consolidation (Morris et al., 1982; Sutherland et al., 1983; Squire, 1992; Nadel and Moscovitch, 1997; VarghaKhadem et al., 1997; Tulving and Markowitsch, 1998; Ferbinteanu et al., 2003; Sutherland, 2005).

The theory of multiple memory systems states that there are different memory systems in the brain that simultaneously process information, but that certain systems are 
optimized for specific types of memory (McDonald et al., 2004). For example, McDonald and White (1993) were able to clearly dissociate three of these systems: the hippocampus was optimized for spatial memory, the amygdala for classical conditioning involving primitive reward circuits and the dorsal striatum was important for stimulusresponse learning (habit formation). Animals with hippocampal lesions were found to be specifically impaired in the spatial memory task, but were able to learn the cue-response task, and conditioned place preference. In contrast, animals with amygdala damage were able to learn cue-response and spatial tasks, but not the conditioned place preference. I took advantage of this dissociation when designing my experiments. To show that the risk factors for $\mathrm{AD}$ were selectively targeting the hippocampus, and affecting this memory system, I used a spatial version of the water maze task to examine hippocampal dependent memory, and one of two hippocampal independent tasks (fear conditioning dependent on the amygdala, or the visible platform version of the water maze task which involves the dorsal striatum) to examine hippocampal independent learning.

\section{Objective of the present thesis}

The series of experiments presented here will begin to validate the multiple combinations of co-factors theory of $\mathrm{AD}$, specifically focusing on the role of the cholinergic depletion as an early event in the aging process that predisposes the brain to additional insults resulting in hippocampal dependent memory loss.

\section{Sub-threshold models of risk factors for $A D$}

The first two studies were conducted to develop sub-threshold models of risk factors for use in validating McDonald's (2002) co-factor theory of $\mathrm{AD}$. To clearly show that the interaction between two risk factors has an effect on learning and memory, it is 
important that neither factor on its own can produce a significant impairment. While there are a number of models that describe the effects of stress, stroke, seizures and circadian disruption on measures of learning and memory, there are no well defined models of these risk factors at a level that does not affect cognitive processes.

The first experiment is a series of pilot studies that establishes the parameters for a sub-threshold model of stress, seizures and stroke. The second experiment examines a novel schedule for repeated phase shifts and recovery sessions that demonstrates hippocampal impairment as the result of chronic phase shifting. This study provides further evidence for circadian disruption as a risk factor for cognitive decline possibly leading to $\mathrm{AD}$. The acute phase shifting schedule presented in this study was validated as a sub-threshold risk factor and used in combination with cholinergic depletion in an additional study.

Cholinergic depletion combined with a secondary risk factor

The next four studies examine the effects of cholinergic depletion of the medial septum combined with a secondary risk factor (either active or passive) on measures of learning and memory. The third experiment shows increased seizure severity and impairment on the spatial version of the water maze task in cholinergic depleted rats given a non-convulsive dose of kainic acid. The fourth experiment found a spatial learning impairment in cholinergic depleted rats subjected to focal ischemia directed at the hippocampus. Both of these groups (cholinergic depletion and seizures and cholinergic depletion and stroke) were able to successfully learn the visible platform version of the water maze. The fifth experiment found impairment in spatial learning and memory that appears in cholinergic depleted rats following chronic stress. This 
impairment appears to be specific to hippocampal functioning as performance on a hippocampal independent task (fear conditioning) remains intact. The sixth experiment shows that the previous findings are not simply the result of multiple sub-threshold insults on the hippocampus, as cholinergic depleted rats subjected to phase shifting are able to re-entrain following the phase shift and learn both water maze and fear conditioning tasks at control levels.

Taken together these experiments describe a role for the septal-hippocampal pathway in the development of $\mathrm{AD}$; possibly by increasing the vulnerability of hippocampal neurons to future insults, or by impairing the capacity of the hippocampus for functional recovery following injury. 


\section{Chapter 2}

A series of pilot studies to determine the threshold for behaviourally sub-threshold stress, seizures and stroke 


\begin{abstract}
A proposed etiological model for Alzheimer's disease $(\mathrm{AD})$ describes the contribution of multiple combinations of risk factors that can lead to variants of age related cognitive decline and/or dementia. Many of these risk factors are present at levels that do not affect learning and memory, but still have the potential to cause neuronal loss or damage. As I was most interested in hippocampal function, I chose to use intact performance on the spatial water maze as a behavioural definition of a "sub-threshold" risk factor. Here I describe protocols for inducing sub-threshold levels of stress, seizures and stroke in the rat. First, I compared different schedules of restraint stress designed to raise corticosterone levels in the rat prior to water maze training and found that a variable restraint schedule was able to elevate corticosterone levels, without affecting the ability of the rat to learn the location of a hidden platform in the water maze. Next, I compared the effects of different non-convulsive doses of kainic acid injected prior to testing on the spatial water maze. No differences were found between groups on water maze performance; however, the highest dosage used $(6 \mathrm{mg} / \mathrm{kg}$ ) was the only group that consistently exhibited non-convulsive seizures as defined by freezing and wet dog shakes. I chose this dose as a reliable way to induce non-convulsive seizures in the absence of behavioural impairment. Thirdly, I compared the effects of infusing different concentrations of Endothelin-1 directly into the hippocampus to induce focal stroke. The lowest dosage used (7.5 pmol) did not impair acquisition of the task and was deemed to be sub-threshold. The importance of using well defined sub-threshold risk factors in a cofactors animal model of $\mathrm{AD}$ reduces the risk of a ceiling effect when combining multiple factors, and allows for the appearance of impairment where there was none previously.
\end{abstract}




\section{Introduction}

The quality of the aging process can be negatively affected by a number of seemingly innocuous, or sub-threshold insults that become more common with age, such as stress, seizures or stroke. These are just three of the many factors that have been identified as putting one at risk for poor aging, dementia and/or Alzheimer's disease (AD) (Breteler et al., 1995; Snowden et al., 1997; McDonald, 2002; Wilson et al., 2006). The cause of $\mathrm{AD}$ is as yet unknown, but one theory suggests that it develops as the result of multiple risk factors that target the hippocampus (Hyman et al., 1982; Ball et al., 1985; McDonald, 2002). The hippocampus is especially vulnerable to ischemia, elevated glucocorticoids, excitotoxicity and oxidative stress; all of which tend to increase in frequency and severity with age (Auer et al., 1989; Reiter, 1995; Sapolsky, 1999; Back et al., 2004). Moreover, the initial memory impairments observed in $\mathrm{AD}$ are those thought to be mediated by the hippocampus (Fleischmann and Gabrieli, 1999) and studies have suggested that early $\mathrm{AD}$ pathology (plaques, tangles and cholinergic depletion) may originate from this region (Ball et al., 1985).

McDonald's (2002) co-factor theory of aging and $\mathrm{AD}$ loosely divides risk factors contributing to $\mathrm{AD}$ into two categories: passive factors and active factors. Regardless of their primary classification (e.g., stress is a passive factor; stroke is an active factor), all of these risk factors can be potentially viewed as sub-tbreshold factors that when presented at a low level have little or no effect on cognitive processes, but in combination, or at a sufficient level can lead to neuronal damage and cognitive impairment. Here, I have used a behavioural definition of sub-threshold that is most applicable to elderly humans. During the normal course of aging, undetected neuronal 
dysfunction as a result of stress, seizure activity or 'silent' stroke can build up; however, unless cognition is impaired, the individual would remain unaware of this damage. Recent imaging studies have detected a significant amount of neuronal damage in the brains of non-demented elderly suggesting there is a threshold at which this undetected brain damage can be expressed as a cognitive impairment (Vermeer et al., 2007).

Understanding the contribution of sub-threshold risk factors to the development of cognitive decline, and specifically $\mathrm{AD}$ is important, as an understanding of the etiology of a disorder is vital to developing effective treatments. The cause of $\mathrm{AD}$ is most likely due to the synergistic effect of two or more sub-threshold risk factors that have little effect on their own. In order to better appreciate the role of these factors in cognitive decline, well defined parameters are required for each factor in an animal model that can be used to exhaustively test the possible interactions, their effects on learning and memory and the potential mechanisms mediating these effects.

Here I present three models of common sub-threshold risk factors: chronic stress, seizure activity and 'silent' stroke. In the first experiment I describe a variable restraint stress procedure that can elevate corticosterone levels in the rat, yet has no effect on spatial memory. In the second experiment, I describe the effects of a non-convulsive dose of kainic acid that does not cause convulsive seizures, or impair water maze performance, and in the third experiment I describe a model of focal ischemia using Endothelin-1 infused into the hippocampus that does not impair spatial memory. 
Experiment 1a: The effect of a chronic variable stress schedule on performance of the water maze task

Exposure to stress stimulates the increase in glucocorticoids [stress hormones; cortisol in humans and corticosterone (CORT) in rodents] which bind to low affinity mineralcorticoid receptors and to the high affinity glucocorticoid receptors found throughout the brain, but most prominently in the hippocampus (Sapolsky, 1999). The number of glucocorticoid receptors in this region makes the hippocampus particularly sensitive to the effects of chronic stress (Sapolsky, 1999). Indeed, chronic restraint stress in rats has been found to induce dendritic atrophy of the CA3 neurons and eventual cell death (Watanabe et al., 1992; Vyas et al., 2002; Sapolsky, 2000). Because of the wellknown role of the hippocampus in learning and memory and correlations between the level of CORT and the quality of cognitive aging in rodents and humans (Lupien et al., 1998; Montaron et al., 2006) many research groups have begun to explore the effects of chronic stress on learning and memory in young rats.

There are many stress procedures currently used to explore the effects of chronic exposure to glucocorticoids on hippocampal integrity and learning and memory. Some of these include restraint stress, chronic mild stress, foot or tail shock, daily injections of CORT or exposure to a predator. Of these, restraint stress is one of the most widely used in behavioural research (Glavin et al., 1994). The duration of the stress period and the length of time rats are restrained vary widely between studies. This variability makes it difficult to accurately assess the effects of stress on learning and memory as different groups have found enhancement (Luine et al., 1994), impairment (Conrad et al., 1996; Luine et al., 1996; Abidin et al., 2004; McLaughlin et al., 2007; Radecki et al., 2007) or 
no effect (Luine et al., 1996; McLaughlin et al., 2007) on spatial memory following various chronic restraint stress procedures. For my experiments I wanted to ensure I had a reliable restraint stress procedure that could elevate CORT levels, but did not impair learning and memory as tested on the spatial version of the water maze.

This experiment was a pilot study designed to examine the effects of various mild chronic restraint stress schedules on water maze performance, and particularly to determine the effect of a novel variable restraint stress paradigm. I stressed rats daily over a two week period for 10 minutes, one hour, or variable lengths of time each day. I predicted that the one hour and variable time schedules would impair learning and memory while the 10 minute schedule would have no effect on water maze performance.

\section{1a. Materials and Methods}

\section{Animals}

Twelve male Long Evans hooded rats (300-400g; CCBN breeding colony) were used for this experiment. They were divided into four groups. Three groups were stressed for 14 days and a fourth group of controls were handled daily. Animals were pair housed with food and water ad libitum, on a 12 hour light/dark cycle with lights off at 19:30. All animals were handled in accordance to guidelines set out by the Canadian Council for Animal Care.

\section{Restraint stress}

To induce chronic stress, rats were subjected to one of three restraint stress procedures. Animals were taken in their home cages to a testing room between 09:0011:00 each day and restrained in Plexiglas cylinders (diameter $6.5 \mathrm{~cm}$, length $15 \mathrm{~cm}$ ) for the prescribed amount of time each day. One group (10 min; $n=3)$ was stressed for 10 
minutes a day for two weeks, the second group ( 1 hour; $n=3$ ) was stressed for one hour each day for two weeks, the third group (Variable; $n=3$ ) was stressed for a different length of time each day $(20,5,60,15,10,30$ or 40 minutes; this cycle was repeated twice for a total of two weeks) and the fourth group (Control; $n=3$ ) was handled briefly each day.

\section{Behavioural testing}

Behavioural testing began 24 hours following the final day of stress.

Water maze: Rapid Acquisition Task

I used a three stage variant of the spatial version of the Morris water task (McDonald et al., 2005). A white plastic circular pool $1.4 \mathrm{~m}$ in diameter and $40 \mathrm{~cm}$ deep was filled to within $20 \mathrm{~cm}$ of the top of the wall with water $\left(20-22^{\circ} \mathrm{C}\right)$ made opaque by adding skim milk powder. The invisible platform was $12 \mathrm{~cm}$ in diameter and made of white Plexiglas with holes drilled into the top of it to provide grip for the animals. During training the platform was submerged $2 \mathrm{~cm}$ under water. Extra-maze cues in the training room included five posters of different sizes and orientations mounted on three of the four walls, a computer rack, a door, an animal rack and the experimenter. Four different start points were used [N, S, E, W (not true compass headings)], equally spaced around the pool. The order was randomly selected for each day and each start point was used twice for day one to four and day six. On day five only the three start points farthest from the platform were used (N, E, S) with each start point repeated five times and the initial start point repeated at the end for a total of 16 trials.

In the first stage (Original location training) rats were trained over four days for eight trials a day, to swim to a fixed, spatial location. The second stage (New location 
training) began twenty four hours after completion of stage one. This stage consisted of new spatial location training as animals were re-trained to swim to the new platform location over sixteen trials within two hours on a single day. Stage two allowed me to ask if rats could learn to go to a new location, during a single intensive training session. The third stage (Re-training to original location) occurred twenty four hours after stage two with the platform returned to its original (stage one) location and each animal was given eight trials. This stage allowed me to ask how the massed new location training during stage two affected re-learning to the original location. The platform location for each stage of training is depicted in Figure 2.1.

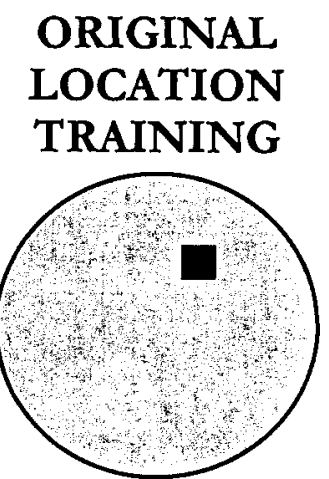

DAY 1-4:

8 TRIALS PER DAY

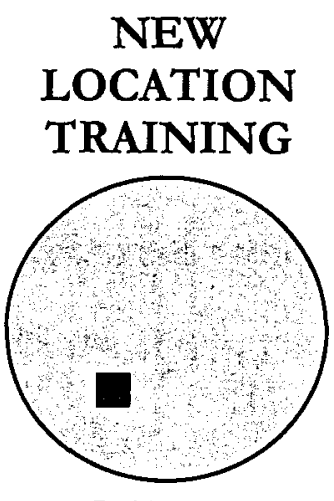

DAY 5:

16 TRIALS
RE-TRAIN TO

ORIGINAL LOCATION

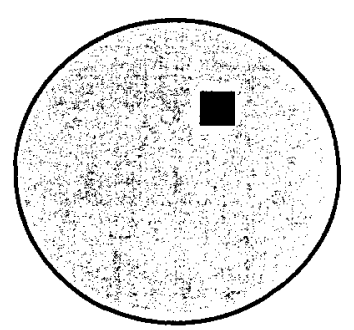

DAY 6:

8 TRIALS

Figure 2.1: Setup for the three stage version of the spatial water maze

This version of the water maze was selected due to its sensitivity to hippocampal damage. The first stage allowed me to identify impairment in acquisition and short term memory of an invariant location. If no differences were apparent, the second stage would test the ability of the hippocampus to rapidly form a novel representation in a familiar context. The third stage measured the rats' ability to re-learn the original location. 
Data collection: A computer based rat tracker (VP118, HVS Image) was used to collect and analyze data obtained from an overhead video camera. For all stages the measure of performance was swim time, defined as the latency between release and escape onto the platform and swim speed. If a rat did not reach the platform 60 seconds after release, a latency of 60 seconds was assigned, and the rat was guided manually to the platform and allowed to remain on the platform for 10 seconds before being removed to a holding cage until the next trial.

\section{1a. Results}

\section{Water maze: Rapid Acquisition Task}

One rat assigned to the 1 hour group died unexpectedly; the following analysis includes data from two rats in this group.

\section{Original location training}

There were no differences between groups in the rats' ability to learn the platform location during original location training (Figure 2.2A). The latency (in seconds) to find the platform was recorded and averaged over the eight daily trials to obtain a single daily latency score for each animal. All groups showed a decrease in latency to find the platform from day one $(23.03+/-2.92$ seconds $)$ to day four $(4.85+/-0.33$ seconds $)$. The daily scores for all four days of acquisition were subjected to a two-way mixed analysis of variance (ANOVA) with Group (10 min versus 1 hour versus Variable versus Control) as the between subjects variable, and Training Day (four levels) as a repeated measure that revealed a significant effect of day $[F(3,21)=35.98, p<0.001]$, but no effect of group $[F(3,7)=1.95, p=0.21]$ or interaction $[F(9,21)=1.43, p=0.24]$. There were no differences in swim speed during this stage of training. A two-way mixed ANOVA with Group as the 
between subjects variable and Swim Speed (four levels) as the repeated measure revealed no effect of group $[F(3,7)=2.37, p=0.16]$, day $[F(3,21)=2.56, p=0.08]$ or interaction $[F(9,21)=1.21, p=0.34]$.

$\boldsymbol{A}$

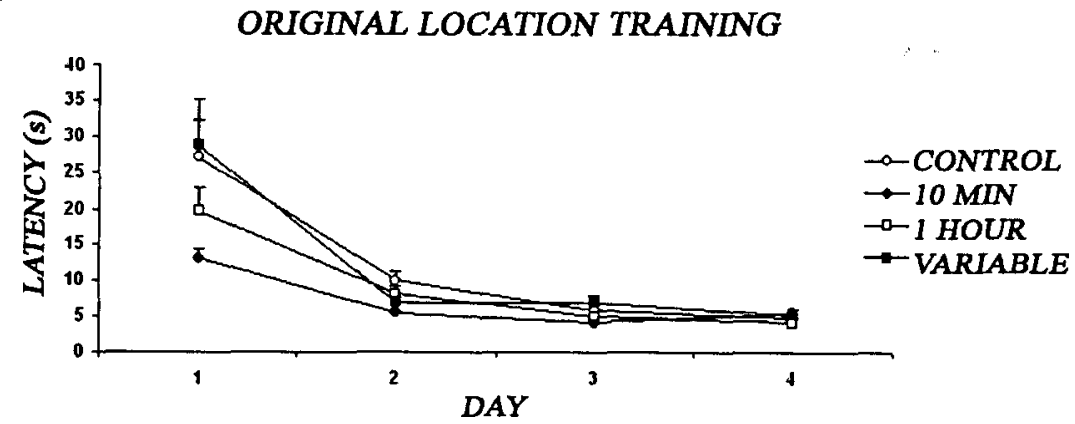

$B$

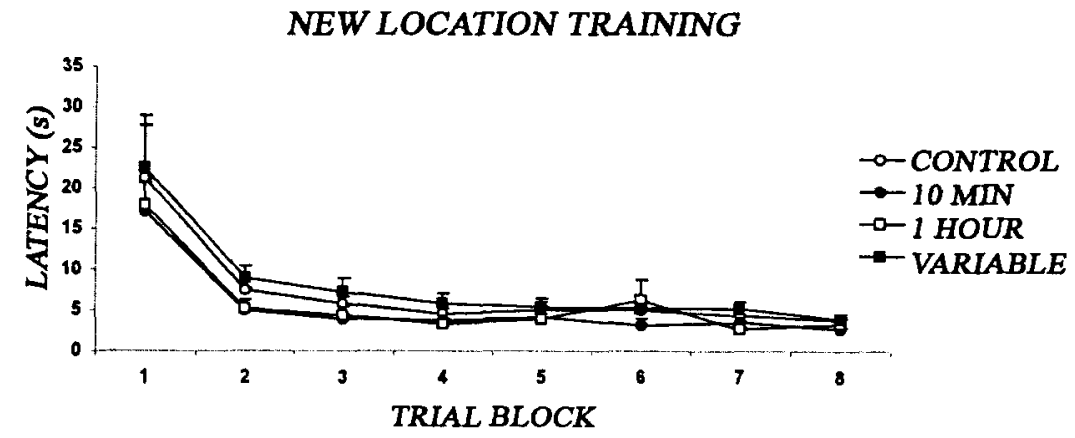

C

RE-TRAIN TO ORIGINAL LOCATION

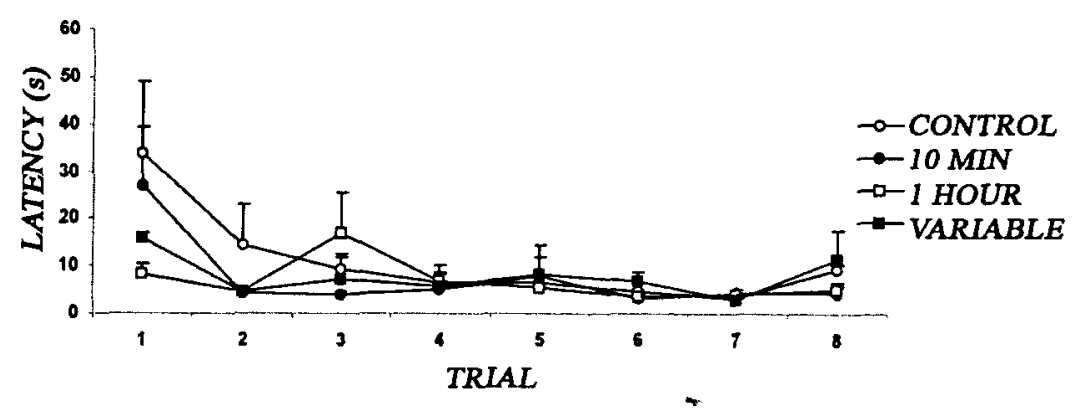

Figure 2.2: Three different restraint stress procedures have no effect on performance in the spatial version of the water maze. All groups were able to learn the location of a hidden platform over four days of training (A), learn a novel location in a single day of massed training $(B)$ and re-learn the original location on day six $(C)$. Data are given as means $+/$ - SEM. 
New location training

The platform was moved to the opposite quadrant of the pool and the latency (in seconds) to find the new platform location was recorded and averaged into eight discrete trial blocks (two trials per block). There were no differences between groups in their ability to rapidly acquire a new platform location on this stage of this task (Figure 2.2B). All groups showed a significant decrease in latency to find the platform from trial block one $(19.93+/-2.41$ seconds) to trial block eight $(3.52+/-0.27$ seconds). A two-way mixed ANOVA with Group as a between subject variable and Trial Block (eight levels) as a repeated measure, was used to assess the new location training and found a significant effect of trial block $[F(7,39)=24.81, p<0.001]$, but no effect of group $[F(3,7)=1.66, p=0.26]$ or interaction $[F(21,49)=0.19, p=0.99]$. There were no differences in swim speed during the new location training. A two-way mixed ANOVA with Group as the between subjects variable and Swim Speed (four levels) as the repeated measure revealed no effect of group $[F(3,7)=1.94, p=0.21]$, trial block $[F(7,49)=1.94, p=0.08]$ or interaction $[F(21,49)=1.06, p=0.41]$.

\section{Re-training to original location}

The platform was returned to the original location (from the first stage) and the latency (in seconds) to find this location was recorded over eight trials on the final day of testing. The results of the re-training trials are displayed in Figure $2.2 \mathrm{C}$ and showed that all groups were able to re-learn the original location on this stage of the water maze task. All groups showed a significant decrease in latency to find the platform from trial one $(20.72+/-5.07$ seconds $)$ to trial eight $(7.87+/-1.76$ seconds $)$. A two-way mixed ANOVA, with Group as the between subjects variable, and Trial (eight levels) as a 
repeated measure revealed a significant effect of trial $[F(7,49)=5.72, p<0.001]$, but no effect of group $[F(3,7)=0.80, p=0.53]$ or interaction $[F(21,49)=1.22, p=0.27]$. There were no differences in swim speed during re-training to original location. A two-way mixed ANOVA with Group as the between subjects variable and Training Day (four levels) as the repeated measure revealed no effect of group $[F(3,7)=1.14, p=0.40]$, trial $[F(7,49)=1.48, p=0.20]$ or interaction $[F(21,49)=0.40, p=0.99]$.

Experiment 1b: The effects of a variable restraint stress procedure on levels of blood borne CORT

I have previously shown that a chronic variable restraint stress procedure has no effect on water maze performance. It is possible that the lack of impairment was due to this procedure being insufficiently stressful and did not elevate CORT levels. This experiment was designed to ensure that this novel variable restraint procedure was able to increase circulating levels of blood borne CORT indicating its suitability as a model of chronic stress.

\section{1b. Materials and Methods}

\section{Animals}

Sixteen male Long Evans hooded rats (300-400g; CCBN breeding colony) were used for this experiment. They were divided into two groups: One group received 14 days of variable stress (Stress; $n=8$ ) and the second group was handled only (Control; $n=8$ ). Animals were housed and cared for as described in Experiment $1 \mathrm{a}$.

\section{Variable stress}

I used the variable restraint stress schedule as described in Experiment 1a. 


\section{Blood collection and analysis of CORT levels}

To assess levels of circulating CORT, rats were placed in a restraint tube and blood samples were obtained by tail nick with a scalpel blade on the final day of stress. Blood was collected from stress rats 30 minutes after being placed in the restraint tube to obtain a peak level of CORT in response to the stressor. Blood was collected from control rats within the first three minutes of being placed in the restraint tube to ensure CORT levels did not have time to significantly increase in response to the brief stress of the procedure. All blood samples $(0.2-0.5 \mathrm{ml})$ were collected into tubes containing $100 \mu \mathrm{l}$ of $10 \%(\mathrm{v} / \mathrm{v})$ ethylenediaminetetraacetic acid (EDTA), placed on ice, centrifuged (7500 rpms for 10 minutes) and the plasma was immediately frozen at $-20^{\circ} \mathrm{C}$. A commercially available ${ }^{125}$ I radioimmunoassay kit (Coat-A-Count Rat Corticosterone, DPC, California) was used for quantifying levels of plasma CORT for each animal.

\section{1b. Results}

\section{Analysis of blood borne CORT levels}

To confirm that my stress procedure produced elevated glucocorticoid levels, I took blood samples on the final day of stress and analyzed them for levels of blood borne CORT. Stressed rats showed a significant elevation in CORT levels following two weeks of variable restraint stress when compared to control rats (Figure 2.3). Data were analyzed using a t-test that found stressed rats had significantly higher levels of blood borne CORT than control rats $[t(14)=8.86, p<0.001]$ 


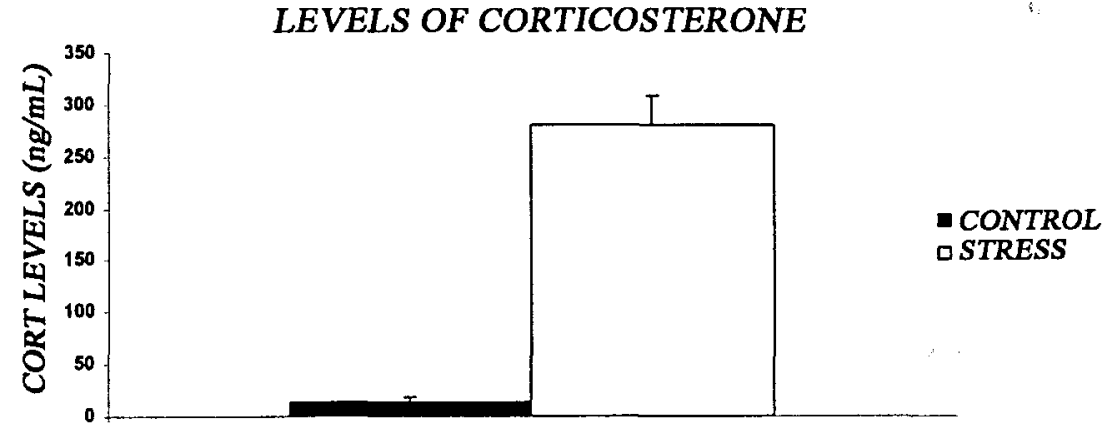

Figure 2.3: The variable restraint stress procedure reliably elevated levels of blood borne CORT measured on the final day of stress compared to non-stressed controls. Data are given as means $+/$ - SEM.

\subsection{Discussion}

Here I show the effects of three mild chronic restraint stress schedules on performance of the water maze task. None of these schedules had any effect on learning and memory as tested on a three stage spatial version of the water maze task shown to be highly sensitive to hippocampal damage in the rat (McDonald et al., 2005; McDonald et al., 2008).

Previous work exploring the effects of chronic restraint on spatial memory has been mixed. Impairment has been reported following one hour daily restraint for 21 days (Abidin et al., 2004), two hours for seven days (Radecki et al., 2005), or two hours for 21 days (Trofimiuk and Braszko, 2008) but other groups have found no effect after two hours for 10 days, two hours for 21 days, six hours for ten days (McLaughlin et al., 2007), or six hours for seven days (Luine et al., 1996). Consistent impairment has been reported following six hours of restraint for 21 days (Luine et al., 1994; Conrad et al., 1996; McLaughlin et al., 2007). The discrepancy between the effects of mild chronic stress (one to two hours for less than two weeks) may be due to differences in the type of 
restraint used (wire mesh, plastic tubes or immobilization bags), rat strain or behavioural test used (water maze, radial arm maze or Y-maze). Future studies are required to fully understand the effects of chronic restraint stress on learning and memory.

There have been no reports to my knowledge examining the effects of extremely short (10 minutes) restraint stress and memory. This length of time has been reported to elevate CORT levels in aged rats (Glavin et al., 1994), although CORT levels are found to increase further with longer durations of restraint (Aragon et al., 1990). I predicted there would be no effect on spatial memory as a result of this mild stress and my results have supported my prediction. The finding of no impairment in my one hour group was surprising as 21 days of one hour restraint is sufficient to cause impairment on water maze (Abidin et al., 2004); however, the studies by Luine et al $(1994,1996)$ have demonstrated that how many days a rat is stressed for is as important as the duration of daily restraint. They reported that seven days of stress (six hours per day) has no effect on performance and 14 days of stress can enhance performance, while 21 days of restraint impairs performance on the radial arm maze (Luine et al., 1994, 1996). The variable restraint stress schedule used here did not affect water maze performance. It is likely two weeks was not long enough, and if I had continued the cycle for an additional week, impairment may have become apparent in these rats. Alternatively, if I increased the daily duration of stress (vary between one to six hours) I may be able to observe an impairment in spatial memory. Although the number of animals used in this pilot was low, additional studies in our laboratory have consistently reported no effect of either one or two weeks of this variable restraint stress schedule on water maze performance 
providing further support for my sub-threshold stress model (Craig et al., 2008; McDonald et al., 2008).

Habituation to stressful experiences is often observed in stress research as exhibited by a decreased CORT response and enhanced return to baseline levels when compared to the CORT secretion profile obtained following acute stress (Magarinos and McEwen, 1995; Gadek-Michalska and Bugajski, 2003; Helm et al., 2004; Marin et al., 2007). In an attempt to prevent habituation, the chronic mild stress procedure (CMS) was developed. This procedure rotates animals through a series of one or two stressors a day (e.g., soiled bedding, saline injection, foot shock, crowding or isolation) that change daily. This procedure has been shown to maintain high levels of CORT throughout the duration of the experiment (Song et al., 2006) and when compared to chronic restraint stress shows significantly less habituation (Maganinos and McEwen, 1995). There have only been two studies exploring the effects of CMS on spatial memory and these follow a similar pattern to single stress procedures. After 10 days of CMS, Gouirand and Matuszewich (2005) reported enhanced water maze performance, while after five weeks of CMS, Song et al. (2006) reported impaired performance. My findings from the variable restraint stress schedule are in agreement as no impairment is observed in the water maze task after two weeks of stress. The advantage to my variable restraint procedure is that it reliably elevates CORT levels and likely minimizes habituation without introducing the variability and potential confounds of using multiple different stressors. Although I did not perform a time course analysis of CORT levels in the current study or take blood from $10 \mathrm{~min}$ or 1 hour rats, I predict that following the variable restraint procedure, rats would exhibit higher levels of blood born CORT than 
those rats exposure to 10 minutes or 1 hour of daily restraint stress. Future studies are required to fully describe the CORT profile induced by this novel stress schedule.

Although all three of my stress schedules had a similar effect on learning and memory, as tested in the water maze task, I chose to use the variable restraint procedure for all future studies. The variation in the length of time restrained each day should minimize habituation making this a useful model of behaviourally sub-threshold chronic stress in rats.

Experiment 2: The effect of various non-convulsive doses of kainic acid on water maze performance

Seizures are a common occurrence in the elderly population, especially in those diagnosed with AD (Hauser et al., 1986; Romanelli et al., 1990; Hesdorffer et al., 1996). The hippocampus is exceptionally sensitive to the excitotoxic damage caused by excessive glutamate released during seizures, due to the number of glutamate receptors found in this region (Ben-Ari, 1985). Patients suffering from epilepsy exhibit hippocampal sclerosis that may be correlated with cognitive decline in this population (Breteler et al., 1995; Thompson and Duncan, 2005; Marques et al., 2007). Animal models of epilepsy and seizures have also shown memory deficits and hippocampal damage similar to those reported in human studies (Cavazos et al., 1994; Gayoso et al., 1994; Gilbert et al., 1996).

Two of the most common animal models of epilepsy are kindling and systemic injection of kainic acid. During kindling, a specific brain region (e.g., hippocampus, amygdala) is subjected to repeated electrical stimulation that results in the development and spread of seizure activity resulting in a heightened susceptibility to seizures in the 
fully kindled animal (Goddard et al. 1969). Kainic acid is a glutamate agonist that has been commonly used as a model for excitotoxic cell death and as an animal model for epilepsy. Systemic injections of kainic acid result in a characteristic response consisting of limbic seizures and a specific pattern of neuronal cell death. The severity of these seizures is dose dependent with concentrations of $10-12 \mathrm{mg} / \mathrm{kg}$ commonly used to induce convulsions and $6 \mathrm{mg} / \mathrm{kg}$ used as a non-convulsive dose (Lothman and Collins, 1981; Gobbo and O'Mara, 2004). Seizure progression follows a reliable pattern with initial freezing and 'staring spells', followed by wet dog shakes, mild automatisms, and eventual fully generalized tonic-clonic seizures that appear within one to two hours of injection (Lothman and Collins, 1981). Interestingly, within the first two weeks after the initial seizure, kainic acid treated rats begin to exhibit spontaneous seizures that persist for months (Hellier et al., 1998, 1999). In addition systemic kainic acid results in a characteristic pattern of neuronal cell death in the hippocampus, amygdala, piriform and entorhinal cortices, (Lothman and Collins, 1981; Ben-Ari, 1985; Sperk, 1995) which is due to over stimulation of glutamate receptors in these regions (Ben-Ari, 1985).

This experiment was designed to determine the effects of various non-convulsive doses of kainic acid on seizure severity and performance on the water maze task. I predicted that none of the doses would impair water maze performance. Furthermore, I predicted that I would observe increased seizure severity with increasing dosages of kainic acid, but none would lead to generalized convulsions. This was a pilot study carried out to describe a dose of kainic acid that could reliably cause non-convulsive seizures (freezing, wet dog shakes) as a sub-threshold risk factor. 


\subsection{Materials and Methods}

\section{Animals}

Twenty four male Long Evans hooded rats (300-400g; CCBN breeding colony) were used for this experiment. They were divided into four groups. Three groups received varying doses of kainic acid intraperitoneally $[1 \mathrm{mg} / \mathrm{kg}$ i.p. $(n=6), 3 \mathrm{mg} / \mathrm{kg}$ i.p. $(n=5)$ and $6 \mathrm{mg} / \mathrm{kg}$ i.p. $(n=6)]$ and the fourth group $(n=7)$ were control animals that received a vehicle injection. Animals were pair housed with food and water ad libitum, on a 12 hour light/dark cycle with lights off at 19:30. All animals were handled in accordance to guidelines set out by the Canadian Council for Animal Care.

\section{Seizures}

All animals were injected with varying doses of kainic acid $(1 \mathrm{mg} / \mathrm{kg}$ i.p., $3 \mathrm{mg} / \mathrm{kg}$ i.p. or $6 \mathrm{mg} / \mathrm{kg}$ i.p.; Ocean Produce International, Halifax, Nova Scotia) dissolved in sterile 1x phosphate buffered saline (PBS) or PBS alone and observed in clear Plexiglas boxes. These doses are considered to be non-convulsive in normal adult rats (Mikulecka et al., 1999; Gobbo and O'Mara, 2004). Rats were observed for the first sixty minutes and scored for seizure severity using a four point scale based on Lothman and Collins (1981): 0-nothing, sleeping; 1-immobility, hunched posture, 'staring'; 2-wet dog shakes; 3-mild limbic convulsions, automatisms; 4-severe limbic convulsions, rearing, bilateral clonus, salivation, loss of postural control. A rat that display behaviours from stages 1-2 was considered to have had a non-convulsive seizure, while rats that exhibited behaviours from stages 3-4 were considered to have had a convulsive seizure.

\section{Behavioural testing}

Behavioural testing began one week following the injection of kainic acid. 


\section{Water maze task}

I used a standard spatial version of the water maze task. The same pool that was described in Experiment 1 was used. Four different start points were used [N, S, E, W (not true compass headings)], equally spaced around the pool. The order was randomly selected for each day and each start point was used twice for a total of eight trials per day.

Rats were trained over six days to swim to a fixed spatial location. On day seven the platform was removed from the pool and each animal was placed in the pool for a 60 second probe trial. On day eight the platform was placed in a new location opposite the original quadrant and rats were given eight trials to learn the new location.

Data collection: A computer based rat tracker (VP118, HVS Image) was used to collect and analyze data obtained from an overhead video camera. For the initial acquisition, and the reversal test on day eight, the measures of performance were swim time, defined as the latency between release and escape onto the platform, and swim speed. If a rat did not reach the platform 60 seconds after release, a latency of 60 seconds was assigned, and the rat was guided manually to the platform and allowed to remain on the platform for 10 seconds before being removed to a holding cage until the next trial. For the probe trial the percentage of time spent in each quadrant of the pool was calculated and used to determine whether rats had formed a memory for the platform location.

\subsection{Results}

\section{Seizures}

Following the injection of $3 \mathrm{mg} / \mathrm{kg}$ or $6 \mathrm{mg} / \mathrm{kg}$ kainic acid, most rats had mild non-convulsive (stage 2) seizures consisting of freezing, and wet dog shakes which are 
common observations during kainic acid induced seizures in rats (Lothman and Collins, 1981). One $3 \mathrm{mg} / \mathrm{kg}$ rat did not display any sign of behavioural seizures, and one $6 \mathrm{mg} / \mathrm{kg}$ only reached a stage 1 seizure, but the rest of the animals in these groups had stage 2 seizures. A few of the rats that received a $1 \mathrm{mg} / \mathrm{kg}$ dose displayed some freezing and wet dog shakes, but $50 \%$ of rats did not exhibit any observable seizure behaviour. Figure 2.4 shows the distribution of seizure severity in each of the dosage groups.

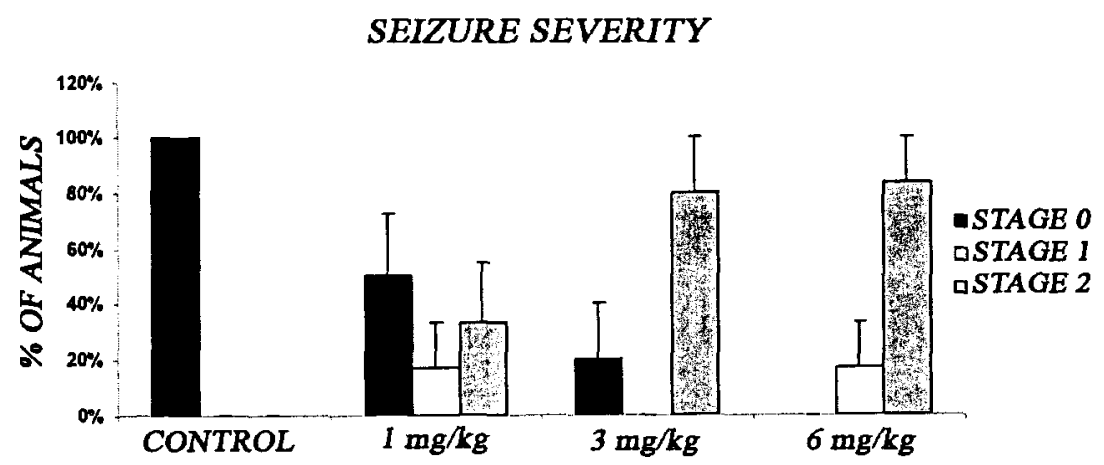

Figure 2.4: Both $3 \mathrm{mg} / \mathrm{kg}$ and $6 \mathrm{mg} / \mathrm{kg}$ doses produced reliable Stage 2 seizures, while the lowest dose $(1 \mathrm{mg} / \mathrm{kg}$ ) resulted in no seizure behaviour in the half the animals. Data are given as means $+/-$ SEM.

\section{Water maze task}

\section{Acquisition}

There were no differences between groups in the rats' ability to learn the platform location during acquisition training (Figure 2.5A). The latency (in seconds) to find the platform was recorded and averaged over the eight daily trials to obtain a single daily latency score for each animal. All groups showed a significant decrease in latency to find the platform from day one $(27.46+/-1.88$ seconds $)$ to day six $(5.64+/-0.41$ seconds). The daily scores for all six days of acquisition were subjected to a two-way mixed 
ANOVA with Group ( $1 \mathrm{mg} / \mathrm{kg}$ versus $3 \mathrm{mg} / \mathrm{kg}$ versus $6 \mathrm{mg} / \mathrm{kg}$ versus Control) as the between subjects variable, and Training Day (six levels) as a repeated measure, and revealed a significant effect of day $[F(5,100)=67.38, p<0.001]$, but no effect of group $[F(3,20)=0.86, p=0.48]$ or interaction $[F(15,100)=0.31, p=0.99]$. There were no differences in swim speed between groups during the six days of acquisition training. A two-way mixed ANOVA with Group as the between subjects variable and Training Day (six levels) as the repeated measure found no effect of group $[F(3,8)=0.20, p=0.89]$, day $[F(5,40)=1.31, p=0.28]$ or interaction $[F(15,40)=0.62, p=0.84]$.

\section{Probe trial}

The platform was removed from the pool and the percentage of time spent in each quadrant was calculated. All rats showed a significant preference for the target quadrant, spending $42.7+/-2.08 \%$ of their time in that quadrant (Figure 2.5B). A two-way ANOVA (Group x Quadrant) revealed no difference between groups on the percentage of time spent in the target quadrant compared to the averaged percentage of time spent in the other three quadrants $[F(3,20)=0.53, p=0.67]$. There were no differences between groups on swim speed during the probe trial. A one way ANOVA found no effect of group $[F(3,20)=1.45, p=0.26]$ on this stage of training. 
$\boldsymbol{A}$ ACQUISITION TRAINING
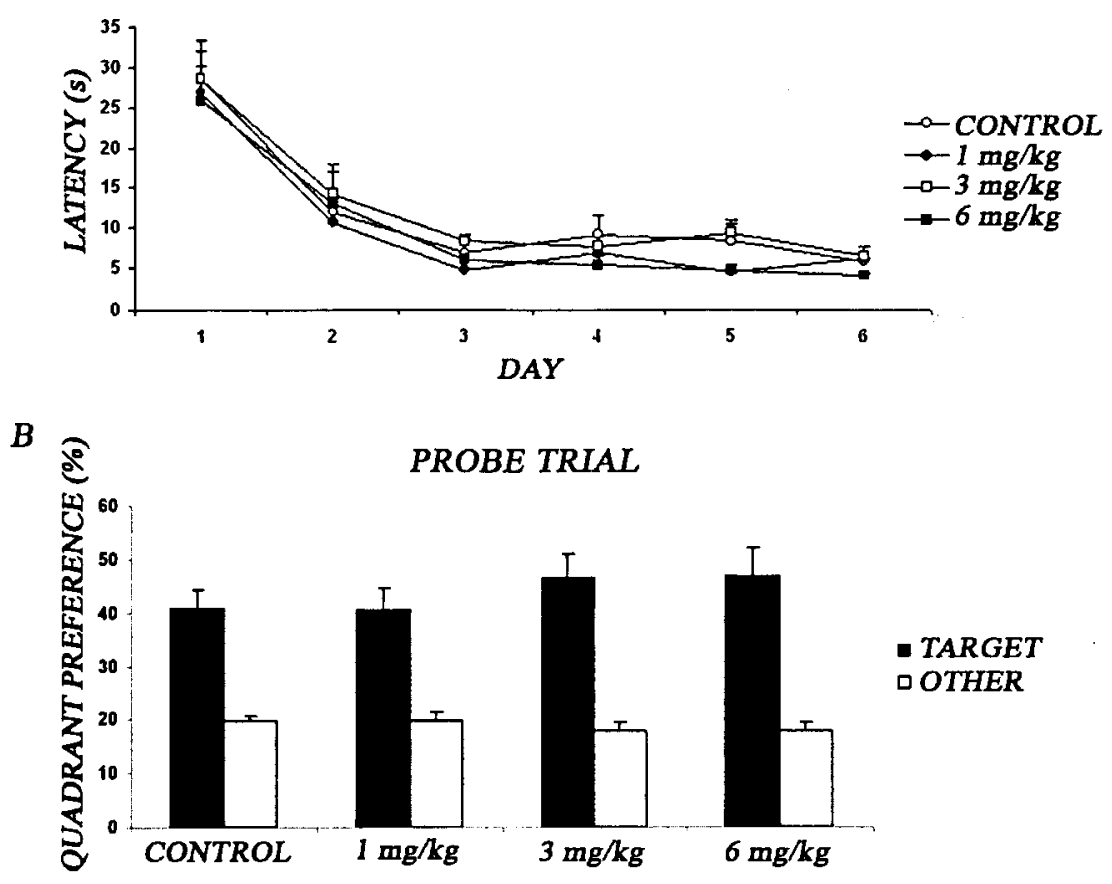

C

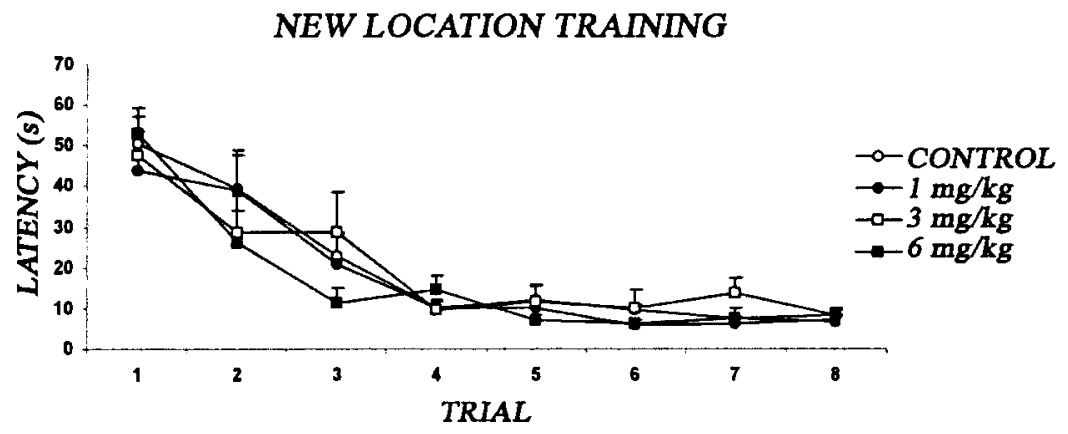

Figure 2.5: Three different non-convulsive doses of kainic acid did not affect performance in the spatial version of the water maze. All groups were able to learn the location of a hidden platform over six days of training (A) and show a preference for the target quadrant during a 60 second probe trial (B). On day eight, all groups were able to learn to find a hidden platform in a novel location (C).-Data are given as means $+/-$ SEM.

Reversal test

On day eight, the platform was returned to the pool and placed opposite the target quadrant. The latency (in seconds) to find this new location was recorded over eight trials on the final day of testing. The results of the reversal are displayed in Figure 2.5C 
showing that all groups were able to learn a new location in a single day. All groups showed a significant decrease in latency to find the platform from trial one $(48.85+/$ 3.18 seconds) to trial eight ( $7.59+/-0.55$ seconds). A two-way mixed ANOVA, with Group as the between subjects variable, and Trial (eight levels) as a repeated measure revealed a significant effect of trial $[F(7,140)=47.62, p<0.001]$, but no effect of group $[F(3,20)=0.28, p=0.84]$ or interaction $[F(21,140)=0.90, p=0.59]$. There were no differences between groups in swim speed on the reversal training day, though all groups increased swim speed over the course of the trials. A two-way mixed ANOVA with Group as the between subjects measure and Trial (eight levels) as the repeated measure and revealed a significant effect of trial $[F(7,56)=2.88, p<0.05]$, but no effect of group $[F(3,8)=0.37, p=0.78]$ or interaction $[F(21,56)=0.76, p=0.76]$. Holm-Bonferroni post hocs revealed faster swim speeds on the final three trials compared to the first two trials $(p ' s<0.05)$.

\subsection{Discussion}

Here I report the effects of three non-convulsive doses of kainic acid on seizure severity and spatial memory in the rat. None of the doses used impaired water maze performance and no convulsive seizures were observed in any of the groups. The $3 \mathrm{mg} / \mathrm{kg}$ and $6 \mathrm{mg} / \mathrm{kg}$ doses consistently induced freezing and staring spells, followed by wet dog shakes, indicative of seizures originating from a hippocampal focus (Damiano and Connor, 1984). The $1 \mathrm{mg} / \mathrm{kg}$ dose occasionally resulted in freezing and wet dog shakes, but this was not consistently observed in all animals.

My findings are consistent with previous reports of non-convulsive seizures on behaviour. Gobbo and O'Mara (2004) tested rats on the water maze two weeks after a 
non-threshold dose of kainic acid $(6 \mathrm{mg} / \mathrm{kg})$ and found no effect on spatial memory. To the best of my knowledge this was the only study exploring the effects of low doses of systemic kainic acid on spatial memory. Mickulecka et al. $(1999,2000)$ found changes in exploratory and anxiety behaviour measured in the open field and elevated plus maze immediately following non-convulsive kainic acid induced seizures; however it is likely these effects were transient and due to the effects of seizure activity in the brain caused by kainic acid. Convulsive doses of kainic acid have been consistently shown to impair spatial memory when tested 10-14 days post seizure, but these effects can be overcome through extensive training (Gayoso et al., 1994; Gobbo and O'Mara, 2004).

Reports on the behavioural effects of non-convulsive seizures are minimal, but there have been some studies examining the behavioural effects of partial kindling where similar findings have been observed. Gilbert et al. (1996) reported no effect on water maze performance in rats that had been kindled to non-convulsive seizures 24 hours prior to training, but in a later paper reported impairment in partially kindled rats that were trained and tested 25-45 minutes after kindling (Gilbert et al., 2000). Sutherland et al. (1997) reported no impairment on the standard version of the Morris water maze, following repeated afterdischarges that did not lead to seizures. Interestingly, they did find a deficit when rats were tested on a more sensitive moving platform variant of the water maze confirming the importance of taking into account the difficulty of behavioural tasks when comparing studies (Sutherland et al., 1997). Leung and Shen (1991) gave rats one week to recover after partial kindling and reported impaired retention in the radial arm maze suggesting that while rats are able to learn a new task (water maze) following partial kindling, the retention of recently learned behaviour may be disrupted. An 
alternate explanation for the impaired performance is that the working memory version of the radial arm maze places a high demand on hippocampal processing and as such is more sensitive to minor hippocampal dysfunction than the standard water maze task. Overall, it appears that 24 hours is sufficient for rats to recover from the effects of partial kindling when tested on the standard version of the water maze, which is consistent with my current finding of no impairment on the water maze task one week after kainic acid injection.

When hippocampal memory impairment is observed following seizures, it may be due to abnormal neuronal activity interfering with the formation of new memories (Buzsaki, 1989; Sutherland et al., 1997). Interictal spikes can be recorded from the hippocampus following partial kindling, but these spikes only persist for a day or two (Leung et al., 1990). A more lasting change (25 days post kindling) can be observed in the averaged evoked potentials in the CA1 which are increased in partially kindled rats (Leung and Shen, 1991). Interictal spikes are also observed following systemic injection of convulsive doses of kainic acid (Hellier et al., 1999), but to the best of my knowledge, it is not yet known whether there are long lasting changes in activity resulting from nonconvulsive doses. Rats treated with convulsive doses of kainic acid begin to exhibit spontaneous seizures that can persist for months (Hellier et al., 1998, 1999). It is possible that these electrophysiological abnormalities may underlie the learning and memory deficits following convulsive seizures in rats.

A second way that seizures can impair learning and memory is through excitotoxic damage to the hippocampus, particularly to the CA1 and CA3 regions that have been shown to be important for learning and memory. Specific lesions to either of 
these regions of the hippocampus can impair performance on the water maze task (Stubley-Weatherly et al., 1996). Convulsive seizures increase levels of excitatory neurotransmitters in the brain, particularly glutamate, which bind to N-methyl-Daspartate (NMDA) receptors in the hippocampus (Fujikawa, 2005). The CA1 and CA3 regions are the most susceptible to excitotoxic damage due to the density of glutamate receptors in these regions. CA2 neurons do not express the same amount of receptors and tend to be spared following seizure activity (Ben-Ari, 1985). Binding of glutamate causes a dramatic increase in intracellular $\mathrm{Ca}^{2+}$ leading to increased apoptotic and necrotic cell death. The non-convulsive seizures observed in the current study were likely not sufficient to damage hippocampal neurons. Though I did not conduct a histological assessment of the brains in the current study, additional experiments in our laboratory have found no evidence of gross hippocampal damage following the $6 \mathrm{mg} / \mathrm{kg}$ dose of kainic acid in the absence of convulsive seizures. This is in agreement with Lothman and Collins (1981) who did not detect hippocampal damage until convulsive seizures had been induced using doses in excess of $7 \mathrm{mg} / \mathrm{kg}$ i.p.

There were no effects on spatial memory in any of my groups receiving low doses of kainic acid; however, there were observable differences between groups in terms of seizure severity. I favored the highest dose $(6 \mathrm{mg} / \mathrm{kg}$ i.p.) for two reasons. First, I could confirm the presence of seizure activity through behavioural manifestations (wet dog shakes) and secondly, it was near to the convulsive threshold allowing for the potential appearance of impairment or neuronal damage when combined with additional factors in McDonald's co-factor model of aging (McDonald, 2002). This dose will be used in future studies as a reliable means of inducing non-convulsive seizure activity in the rat brain. 


\section{Experiment 3: The effects of focal sub-threshold dose of Endothelin-1 on water maze performance}

Ischemic stroke is the most common form of stroke and is caused by an interruption of the blood flow to the brain due to a transient or permanent blockage of the cerebral arteries. The specific brain region(s) deprived of oxygen undergo cell death resulting in an infarct (area of dead tissue); the size and location of which can be correlated with neurological deficits (Durukan and Tatlisumak, 2007). Not all strokes result in obvious symptoms and are referred to as 'silent' strokes which occur commonly in the elderly population. These asymptomatic events can be detected as small infarcts on magnetic resonance imaging (MRI) or computerized tomography (CT) scans. Approximately $20 \%$ of the healthy elderly population is found to have experienced a 'silent' stroke in their lifetime, with this prevalence increasing in patients that have suffered a stroke or dementia (Vermeer et al., 2007).

The hippocampus is especially vulnerable to the effects of ischemia and cerebral hypoperfusion (Schimdt-Kastner and Freund, 1991; Farkas and Luiten, 2001). Both global ischemia and lacunar stroke have been found to preferentially target the hippocampus resulting in increased levels of apoptosis and hippocampal atrophy (Kirino and Sano, 1984; Honkaniemi et al., 1996; Back et al., 2004; Grau-Olivares et al., 2007). Stroke can be either global or focal in nature, referring to the regions of the brain affected. Most studies are designed to detect impairment and recovery of a specific behaviour (e.g., skilled forelimb reaching, spatial memory, sensory deficits, etc) and for these studies, focal ischemia is preferred due to the specificity of the lesion. There are several models of focal ischemia currently used in animal research [middle cerebral 
artery occlusion, photothrombosis and administration of vasoconstrictive agents, such as Endothelin-1 (ET-1)], each of which has their own advantages and disadvantages. I was interested in creating a specific focal lesion of a subcortical structure (the hippocampus) that could be manipulated in a dose dependent fashion. For these reasons I chose to use the potent vasoconstrictor ET-1 in my model.

Topical, intracortical, or perivascular administration of ET-1 reduces blood flow to a specific region until it can be metabolized and allow normal blood flow to resume. The resulting infarct is highly reliable and its size is dose dependent. This technique was first developed by Fuxe et al. (1992) who infused small amounts of ET-1 into the striatum to create a focal lesion that was thought to be ischemic in nature. This lesion could be prevented by the co-administration of a vasodilator. In addition, application of ET-1 to neuronal cultures in vitro was not found to be neurotoxic, further supporting the idea that the damage resulting from ET-1 was ischemic in nature (Nikolov et al., 1993). Intracortical infusions of ET-1 have been used to damage the sensory and/or motor cortex (Adkins et al., 1993; Gilmour et al., 2004), the striatum (Fuxe et al., 1992) or a combination of motor cortex and striatum (Windle et al., 2006). The resultant lesions were found to be associated with motor deficits comparable to those observed with aspiration or excitotoxic lesions of the same region (Gilmour et al., 2004).

This experiment was designed to examine the effects of varying doses of ET-1 infused directly into the hippocampus on performance on the water maze task. I predicted a dose dependent response to ET-1 with rats receiving the largest dose being the most impaired on the water maze task and rats receiving the lowest dose having little or no impairment when compared to controls. This was a pilot study carried out to describe a 
dose of ET-1 that can induce mild focal ischemia with the purpose of developing stroke as a sub-threshold risk factor for future studies.

\subsection{Materials and Methods}

\section{Animals}

Twenty nine male Long Evans hooded rats (300-400g; CCBN breeding colony) were used for this experiment. Rats were divided into four groups: Low dose (7.5 pmol; $n=8$ ) rats were infused with $7.5 \mathrm{pmol}$ of ET-1 (human porcine, Sigma), medium dose (30 pmol; $n=6)$ rats were infused with 30 pmol of ET-1, high dose (60 pmol; $n=7)$ rats were infused with 60 pmol of ET-1 and control (Control; $n=8)$ rats were infused with saline. Animals were pair housed with food and water ad libitum, on a 12 hour light/dark cycle with lights off at 19:30. All animals were handled in accordance to guidelines set out by the Canadian Council for Animal Care.

\section{Surgery}

Surgery was performed under Isoflurane anaesthesia $(4 \%$ with $2.0 \mathrm{~L} / \mathrm{min}$ of oxygen for induction and $2 \%$ after surgical plane was established) in a standard stereotaxic apparatus. An incision was made in the scalp and periosteum down the midline. The fascia was pushed to the edges of the skull with a sterile gauze swab and the skin retracted to expose the skull surface. Holes were drilled into the skull using a 1 $\mathrm{mm}$ drill bit attached to a high speed drill. The vasoconstrictor ET-1 was used to induce a focal stroke in the hippocampus. I lowered a 30 gauge cannula, through holes drilled in the skull in to the dorsal (AP -4.1; $\mathrm{ML}+/-3.0 ; \mathrm{DV}-3.7 \mathrm{~mm}$ from bregma and the skull respectively) and ventral hippocampus (AP -5.2; $\mathrm{ML}+/-5.3$; DV -7.0 $\mathrm{mm}$ from bregma and the skull respectively) and bilaterally infused ET-1 $(7.5,30$ or 60 pmol dissolved in 
sterile saline) at a rate of $0.1 \mu \mathrm{l} / \mathrm{min}$ using a Harvard mini-pump. Each animal received a total volume of $5 \mu 1$ to each site. The cannula was then left in place for 10 minutes after each infusion to ensure diffusion of the toxin. Sham operated animals underwent an identical procedure, but were infused with sterile saline instead of ET-1. Following surgery animals were given buprenorphine (Temgesic; $1 \mathrm{mg} / \mathrm{kg}$ subcutaneously) as an analgesic.

\section{Behavioural testing}

Behavioural testing began one week following the infusion of ET-1.

\section{Water maze task}

I used a standard spatial version of the water maze task. The same pool that was described in Experiment 1 was used. Four different start points were used [N, S, E, W (not true compass headings)], equally spaced around the pool. The order was randomly selected for each day and each start point was used twice for a total of eight trials per day. Rats were trained over four days to swim to a fixed spatial location.

Data collection: A computer based rat tracker (VP118, HVS Image) was used to collect and analyze data obtained from an overhead video camera. The measure of performance was swim time, defined as the latency between release and escape onto the platform and swim speed. If a rat did not reach the platform 60 seconds after release, a latency of 60 seconds was assigned, and the rat was guided manually to the platform and allowed to remain on the platform for 10 seconds before being removed to a holding cage until the next trial. 


\subsection{Results}

\section{Behavioural testing}

The three different doses of ET-1 had dramatically different effects on the ability of the rat to learn the location of a hidden platform during training. Rats receiving 30 or $60 \mathrm{pmol}$ were impaired on all days of training, while rats receiving the 7.5 pmol dose did not differ from controls (Figure 2.6). On the final day of training controls and $7.5 \mathrm{pmol}$ rats were finding the platform in $7.5+/-1.2$ seconds and $6.9+/-0.8$ seconds respectively, while 30 and 60 pmol rats were taking $16.2+/-1.4$ seconds and $25.7+/-2.5$ seconds respectively, to find the platform location. A two-way mixed ANOVA with Group (Control versus $7.5 \mathrm{pmol}$ versus $30 \mathrm{pmol}$ versus $60 \mathrm{pmol}$ ) as the between subjects variable and Training Day (four levels) as the repeated measure revealed a significant effect of group $[F(3,25)=49.69, p<0.001]$, of day $[F(3,75)=53.72, p<0.001]$ and an interaction $[F(9,75)=3.69, p<0.001]$. The interaction was further broken down using planned comparisons that found no difference between control and 7.5 pmol rats on any of the training days $(p<0.05)$. Rats receiving $30 \mathrm{pmol}$ took longer than $7.5 \mathrm{pmol}$ rats on days three and four $(p<0.05)$, while $60 \mathrm{pmol}$ rats took significantly longer than all other groups on all days $(p<0.05)$. There were no differences in swim speed between the groups. A two-way mixed ANOVA with Group as the between subjects variable and Training Day (four levels) as the two-way mixed found no effect of group $[F(3,25)=1.43$, $p=0.26]$, of day $[F(3,75)=0.66, p=0.58]$ or interaction $[F(9,75)=1.15, p=0.34]$. 


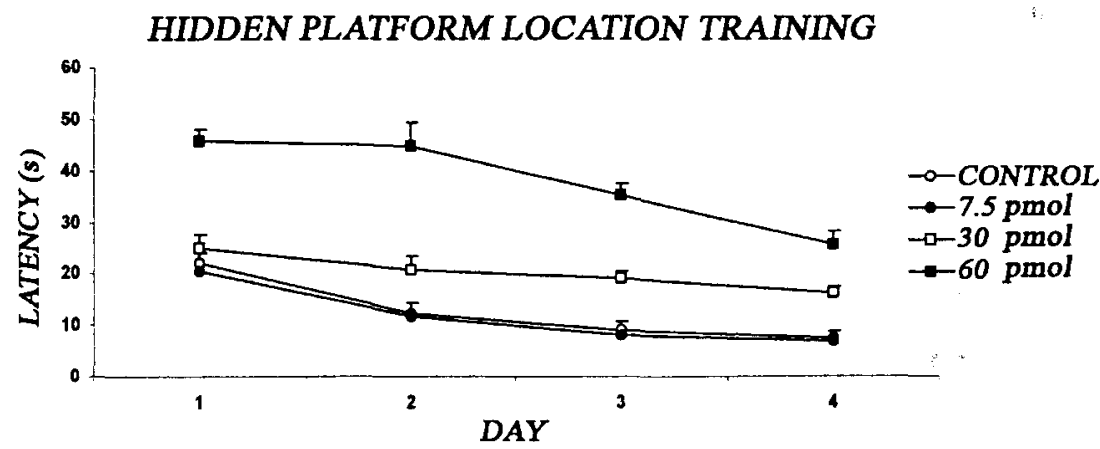

Figure 2.6: The lowest dose of ET-1 (7.5 pmol) did not impair acquisition training in the water maze while the medium ( $30 \mathrm{pmol})$ and high $(60 \mathrm{pmol})$ doses resulted in significantly longer latencies to find the hidden platform compared to the low dose or control rats. Data are given as means $+/$ - SEM.

\subsection{Discussion}

Here, I demonstrate a dose dependent effect of a hippocampal infusion of ET-1 on spatial memory as assessed during acquisition training in a hidden platform version of the Morris water maze. All groups were able to swim well and actively searched for the hidden platform, but the $60 \mathrm{pmol}$ and $30 \mathrm{pmol}$ groups were severely impaired in their ability to learn the platform location. In contrast, rats receiving 7.5 pmol were indistinguishable from controls and rapidly learnt the location of the hidden platform.

This is the first study looking at the behavioural effects of using ET-1 to damage the hippocampus. Previous studies have used ET-1 to damage motor or sensorimotor cortex and have reported region specific impairment in sensation and forelimb use (Adkins et al., 1993; Gilmour et al., 2004). Moreover, Fuxe et al. (1992) has reported a dose dependent effect on lesion size with greater damage resulting from higher doses, which would explain the results in the current study. The acquisition deficit observed in the groups receiving the two largest doses of ET-1 is comparable to previous research on stroke and spatial memory. Middle cerebral artery occlusion causes damage to the 
hippocampus and select cortical areas and can produce impairment on both acquisition and retention of platform location in the water maze task (Markgraf et al., 1992; Yonemori et al., 1996; Luo et al., 2007, but see Karhunen et al. 2006). This deficit on the water maze task following stroke can be improved by treatment with anti-inflammatory drugs (Liu et al., 2006), voluntary wheel running (Luo et al., 2007) or enriched environment (Dahlquist et al., 2004).

Cerebral hypoperfusion is another model of stroke that reduces blood flow to the cortex and hippocampus. This model does not cause extensive damage to the brain, save for a gradual loss of CA1 neurons in the hippocampus (Farkas and Luiten, 2001). Following chronic hypoperfusion, spatial memory is impaired in rats tested on the water maze (de la Torre et al., 1992; Pappas et al., 1996; de Jong et al., 1999). The cognitive effects of mild or sub-threshold stroke have not been extensively studied, but due to the sensitivity of CA1 neurons of the hippocampus, any observed impairment would likely be revealed in tests of hippocampal processing. Auer et al. (1989) briefly induced low level ischemia in rats that resulted in a $50 \%$ loss of CA1 neurons, and found no effect on the standard version of the water maze; however, rats were impaired in a more difficult moving platform version.

I was unable to conduct a histological analysis of lesion size in the current study, so a correlation between lesion size and performance could not be determined. I predict that I would have found minimal damage to the hippocampus in the low dose group and extensive cell death in groups receiving the higher doses. Additional experiments in our laboratory confirmed this prediction by finding minimal damage surrounding the infusion site (Driscoll et al., 2007; McDonald et al., 2008), or a moderate amount of neuronal cell 
death throughout the hippocampus (Craig et al. submitted). Further experiments testing this model on water maze performance have suggested a relationship between lesion size and performance. McDonald et al. (2008) and Driscoll et al. (2007) reported minimal hippocampal damage and no impairment in spatial memory following sub-threshold stroke, and Craig et al. (submitted) reported a mild impairment in the water maze task in rats with moderate hippocampal damage.

The lowest dose (7.5 pmol) of ET-1 used in the current study did not impair acquisition of the water maze task. I chose this dose for future studies to better model the 'silent' infarcts commonly observed in the elderly (Snowden et al., 1997; Vermeer et al., 2007). Although the design of this pilot study was unable to demonstrate a lack of impairment on all stages of the water maze task, additional experiments using this model have since confirmed that while there is some inherent variability to this model, overall spatial memory remains at or near control levels allowing this model to be behaviourally defined as a sub-threshold risk factor.

\section{Conclusion}

I have presented three animals models of sub-threshold risk factors (stress stroke and seizures) that do not impair hippocampal learning and memory as tested on the water maze task. I have discussed a role for these risk factors in the development of $\mathrm{AD}$, and more importantly the potential consequences of behaviourally sub-threshold levels of these risk factors. These models will prove useful in continuing research designed to further understand the etiology of $\mathrm{AD}$. Future studies will explore the effects of combining these risk factors with each other, and others to identify unique "sub-types" of $\mathrm{AD}$ that would respond optimally to individualized treatments. 


\section{Chapter 3}

Chronic circadian disruption impairs hippocampal memory in the rat

Modified from a paper published in Brain Research Bulletin, 76 (1-2):141-151 by Laura A. Craig and Robert J. McDonald in 2008 


\begin{abstract}
Circadian related disorders and alterations in sleep-wake patterns are common complaints in the elderly, especially those diagnosed with Alzheimer's disease. The negative physical and psychological effects resulting from chronic circadian disruption are numerous and appear to be positively correlated with the length of time an individual has suffered from a circadian disorder. In the current paper, I explore the effects of acute and chronic disruption of circadian rhythms on memory using a phase shifting schedule that can continually challenge the rats' circadian system by using repeated phase shifts and recovery sessions. I demonstrate a significant learning and memory deficit on a spatial version of the water maze task in the chronically phase shifted, but not in the acutely phase shifted animals. Moreover, I find no impairment in fear conditioning suggesting that chronic phase shifting predominantly affects hippocampal memory. I propose that chronic circadian disruption may play a role in the development of age related cognitive deficits and dementia in the elderly.
\end{abstract}




\section{Introduction}

Changes in circadian rhythms and deterioration of sleep-wake patterns are some of the most common complaints in the elderly population, especially those patients suffering from dementia or Alzheimer's disease $(\mathrm{AD})$. Indeed, this is the number one cause of institutionalization for demented patients as caretakers at home find it increasingly difficult to manage their patients' frequent night time awakenings (Pollak and Perlick, 1991; Hatfield et al., 2004). An interesting link between circadian disruption and cognitive impairment has previously been reported in both human (Bonnet, 1989) and non-human animals (Fekete et al., 1985; Stone et al., 1997; Antoniadis et al., 2000). Changes in rhythmicity, such as increased night time activity, combined with frequent day time napping, and 'sundowning' (a tendency for patients to become increasingly agitated in the late afternoon/evening) have been consistently reported in $\mathrm{AD}$ patients (Witting et al., 1990; van Someren et al., 1996).These disruptions of the circadian system could play a role in the cognitive deficits observed in $\mathrm{AD}$.

Circadian rhythms are controlled largely by the suprachiasmatic nucleus (SCN) in the brain. The SCN acts as an internal pacemaker, driving a daily rhythm with a period of near twenty four hours although input from light, meals, and social contacts act as cues to set the clock to a precise twenty four hour period (Antle and Mistleberger, 2005). Damage to the SCN in rodents completely abolish these daily rhythms, but rodents can recover normal rhythmicity following the transplantation of healthy SCN tissue confirming the importance of this nucleus as a circadian clock (Ralph et al., 1990; Hurd et al., 1998). Considering the importance of the SCN to the maintenance of circadian rhythms, it is no surprise that individuals suffering from chronic circadian disturbances, 
such as the aged population, and especially patients with $\mathrm{AD}$, show dramatic changes in the structure and function of this region. These changes include reduction in volume and cell number in the $\mathrm{SCN}$ and changes in the rhythmicity and expression of vasopressin producing neurons, suggesting a neuroanatomical basis for the decline in circadian rhythmicity in the aged population (Swaab et al., 1985; Frankland et al., 1998).

The aged non-human animal population also tends to show impairments in their circadian rhythms. In fact, Antoniadis et al. (2000) have demonstrated that in hamsters, the extent of circadian disruption in aged animals is a better predictor of age related cognitive decline than age alone. A wide variety of cognitive processes can also be affected by phase shifting. Phase shifting is a procedure that actively changes one of the external time cues (e.g., advancing the time of lights off by a set number of hours) in order to reset the SCN, causing a healthy animal to modify its behaviour and adapt to the novel schedule. Devan et al. (2001) have shown that phase shifting for six days during acquisition of the water maze task, interferes with long term memory recall for the platform location two weeks later. Other researchers have found impairments in passive and active avoidance following phase shifting (Tapp and Holloway, 1981; Fekete et al., 1985). These studies suggest a link between cognitive impairments and circadian disruption, especially in the area of memory recall, but much more work is required in this area to fully understand the nature of this relationship.

There have been several controlled studies in humans and non-human animals reporting that an acute disruption to circadian rhythmicity can result in memory deficits, specifically those involved in recall. However, the evidence supporting impairments in individuals suffering from chronic circadian disruption tends to predominantly focus on 
human populations, either individuals working shift work [who are specifically selected to be healthier, and more fit to handle the rigors of shift work (Knutsson, 2004)], or aged patients with chronic circadian disorders who often have pre-existing mental health complaints [making it difficult to separate cognitive impairments caused by circadian disruption and those caused by Alzheimer's disease, or dementia (van Someren et al., 2000)]. Exploration into the effects of chronic circadian disruption on learning and memory in both young and aged populations is required to begin to understand the nature of the relationship between circadian disruption and memory. Here I use a phase shifting procedure that allows me to maintain rats in a state of circadian disruption for varying amounts of time in a controlled environment. This will help me to explore the consequences of chronic circadian disruption on cognitive processes and its potential links to physiological, psychological and neurodegenerative disorders. In the current study I demonstrate the effects of acute and chronic circadian disruption on learning and memory in the rat.

\section{Materials and Methods}

\section{Animals}

Thirty seven male Long Evans hooded rats (300-400g) obtained from Charles River (Saint-Constant, PQ) were used for all studies. They were divided into three groups: Acutely phase shifted rats (Acute; $n=8$ ), chronically phase shifted rats (Chronic; $n=8)$ and control rats for water maze testing (Control; $n=7$ ) and control rats for fear conditioning (Control; $n=14)$. Both groups of phase shifted rats $(n=16)$ were housed individually in polypropylene cages equipped with a $42.5 \mathrm{~cm}$ diameter running wheel, with food and water ad libitum, on a 12 hour light/dark cycle, with variable times of light 
offset. Control rats $(n=21)$ were pair housed in standard shoebox housing with food and water ad libitum, on a 12 hour light/dark cycle with lights off at 19:30. All animals were handled in accordance to guidelines set out by the Canadian Council for Animal Care.

\section{Circadian rhythms}

In order to create a state of circadian disruption I used a two stage phase shifting session which lasted 16 days. Acutely phase shifted rats underwent a single session (16 days), while chronically phase shifted rats underwent four consecutive sessions (64 days). Control rats remained on a constant 12 hour light/dark schedule for the duration of the experiment with lights off at 19:30.

\section{Phase Shifting}

Each phase shifting session was made up of two distinct stages: phase shifting and recovery. Animals were initially phased advanced by three hours a day for six consecutive days. This phase advancement was followed by ten days of re-entrainment to allow the animals a partial recovery [ten days is half the normal re-entrainment time required in this paradigm (Deven et al., 2001)]. After this partial re-entrainment, animals were phase advanced, a second time, for three hours a day for another six days, then allowed a second recovery (Table 3.1 shows the time the lights were turned off during the phase shifting procedure). This design allowed me to continually challenge the circadian system for the duration of my experiment.

Data Collection: Phase shifted animals were housed individually in polypropylene cages equipped with a $42.5 \mathrm{~cm}$ diameter running wheel attached via a microswitch to a computer. Activity (wheel running) was monitored continuously using a ClockLab data 
analysis and collection system for circadian rhythms (ActiMetrics Software, Wilmette, IL). Actograms for each animal were double plotted to clearly show general patterns of activity. Daily onsets of activity and total activity for each animal were obtained using the ClockLab analysis program (ActiMetrics Software). The activity onsets were determined for the six days prior to the start of phase shifting session (baseline) and for the six days following each phase shifting session (post 1-4; post 1 refers to the six days following the first phase shifting session, post 2 refers to the six days following the second phase shifting session, etc.). The difference between the actual time of lights off and the onset of activity was calculated, averaged over the six days and used to describe the phase angle of entrainment. A delayed phase angle was negative, and referred to activity onsets occurring after the time of lights off, while an advanced phase angle was positive, and referred to activity onsets proceeding the time of lights off. Activity was recorded in wheel revolutions per minute, and determined for the light phase (light activity) and the dark phase (dark activity). To control for inter-rat variability in total activity, light activity was expressed as a percentage of total activity and used as a measure of rhythm fragmentation. This proportion of light activity was averaged for the six days prior to the start of phase shifting session (baseline) and for the six days following each phase shifting session (post 1-4). 
Table 3.1: Schedule of phase shifting to induce chronic circadian disruption

\begin{tabular}{cc}
\hline DAY & \\
\hline 1 & Lights off at $16: 30$ \\
2 & Lights off at $13: 30$ \\
3 & Lights off at $10: 30$ \\
4 & Lights off at $07: 30$ \\
5 & Lights off at $04: 30$ \\
6 & Lights off at $01: 30$ \\
$7-16$ & Re-entrainment: Lights off at $22: 30$ \\
17 & Lights off at $19: 30$ \\
18 & Lights off at $16: 30$ \\
19 & Lights off at $13: 30$ \\
20 & Lights off at $10: 30$ \\
21 & Lights off at $07: 30$ \\
22 & Lights off at $04: 30$ \\
$23-32$ & Re-entrainment: Lights off at $01: 30$ \\
33 & Lights off at $22: 30$ \\
34 & Lights off at $19: 30$ \\
35 & Lights off at $16: 30$ \\
36 & Lights off at $13: 30$ \\
37 & Lights off at $10: 30$ \\
38 & Lights off at $07: 30$ \\
$39-48$ & Re-entrainment: Lights off at $04: 30$ \\
49 & Lights off at $01: 30$ \\
50 & Lights off at $22: 30$ \\
51 & Lights off at $19: 30$ \\
52 & Lights off at $16: 30$ \\
53 & Lights off at $13: 30$ \\
54 & Lights off at $10: 30$ \\
$55-64$ & Re-entrainment: Lights off at $07: 30$ \\
\hline
\end{tabular}




\section{Behavioural testing}

Behavioural testing occurred following the final ten day re-entrainment of the phase shifting procedure at the equivalent circadian phase for each group. Rats were tested on a six day version of the water maze task, followed by three days of testing on a fear conditioning task. The acutely phase shifted group of rats began behavioural testing after one session (16 days) at 13:30 (three hours after lights on), and the chronically phase shifted group of rats were tested after four consecutive sessions (64 days) at 22:30 (three hours after lights on). The control group of rats were tested at 10:30 (three hours after lights on).

Water maze: Rapid Acquisition Task

I used a three stage variant of the spatial version of the water maze task (McDonald et al., 2005). A white plastic circular pool $1.4 \mathrm{~m}$ in diameter and $40 \mathrm{~cm}$ deep was filled to within $20 \mathrm{~cm}$ of the top of the wall with water $\left(20-22^{\circ} \mathrm{C}\right)$ made opaque by adding skim milk powder. The invisible platform was $12 \mathrm{~cm}$ in diameter and made of white Plexiglas with holes drilled into the top of it to provide grip for the animals. During training the platform was submerged $2 \mathrm{~cm}$ under water. Extra-maze cues in the training room included five posters of different sizes and orientations mounted on three of the four walls, a computer rack, a door, an animal rack and the experimenter. Four different start points were used [N, S, E, W (not true compass headings)], equally spaced around the pool. The order was randomly selected for each day and each start point was used twice for day one to four and day six. On day five only the three start points farthest from the platform were used $(\mathrm{N}, \mathrm{E}, \mathrm{S})$ with each start point repeated five times and the initial start point repeated at the end for a total of 16 trials. 
In the first stage (Original location training) rats were trained over four days for eight trials a day, to swim to a fixed, spatial location. Twenty-four hours after completion of stage 1, the second stage (New location training) began. This stage consisted of new spatial location training as animals were re-trained to swim to the new platform location over sixteen trials within two hours on a single day. Stage 2 allowed me to ask if rats could learn to go to a new location, during a single intensive training session. The third stage (Re-training to original location) occurred twenty four hours after stage 2 with the platform returned to its original (stage 1) location and each animal was given eight trials. This stage allowed me to ask how the massed new location training during stage 2 affected relearning to the original location. The platform locations for each stage of this task are depicted in Figure 2.1.

This version of the water maze was selected due to its sensitivity to hippocampal damage. The first stage allowed me to identify impairment in acquisition and short term memory of an invariant location. If no differences were apparent, the second stage would test the ability of the hippocampus to rapidly form a novel representation in a familiar context. The third stage tested the strength of each representation, as an unimpaired rat would clearly demonstrate a memory for both locations with a preference for the most recent (McDonald et al., 2005).

Data collection: A computer based rat tracker (VP118;HVS Image) was used to collect and analyze data obtained from an overhead video camera. The measures of performance were swim time, defined as the latency between release and escape onto the platform and path length to the platform. If a rat did not reach the platform 60 seconds after release, a latency of 60 seconds was assigned, and the rat was guided manually to the platform and 
allowed to remain on the platform for 10 seconds before being removed to a holding cage until the next trial. An additional analysis was conducted on the first swim of re-training to determine the first quadrant entered, which was used as a measure of platform preference (new versus original).

\section{Fear conditioning to tone and context}

The three day fear conditioning procedure used here was designed to test hippocampal independent learning and memory on two related tasks: fear conditioning to tone (cue) and to context. Animals were placed into Context A (an operant chamber, metal walls, bars on floor, scented with Quatsyl@-D Plus disinfectant) and allowed to habituate for two minutes. They were presented with a 10 second tone $(80 \mathrm{db})$, followed by a two second shock (1.0 mA). This tone-shock pairing was repeated every 70 seconds for five repetitions. Sixty seconds following the final shock, the animals were removed from the chamber, and returned to their home cage. Twenty four hours later the animals were placed in a novel context, Context B (Plexiglas walls, bedding on the floor, unscented), and after two minutes of habituation, were presented with an eight minute tone $(80 \mathrm{db})$, with no shock and scored for freezing behaviour, as a measure of fear. Freezing behaviour is a reliable measure of fear, and was defined as a period of complete immobility except for breathing movements in the animal (Blanchard and Blanchard, 1969). Forty eight hours later animals were returned tơ Context $\mathrm{A}$ for a five minute trial (no shock, no tone) to assess freezing behaviour in response to the original conditioning context. 


\section{Results}

One Acute rat died for unknown reasons prior to phase shifting and behavioural testing; the following analyses include data from seven rats in this group.

\section{Circadian rhythms}

Circadian rhythms were continuously recorded throughout the study using a running wheel connected to a computerized data collection system (due to minor technical difficulties with equipment used in the data collection process some data for individual rats was lost. On that day the rat would be removed from the corresponding circadian rhythm analysis; one Acute rat was removed from the entire analysis due to multiple missed days). A representative actogram is shown from each group illustrating the initial entrainment, phase shifting session(s) and recovery session(s) for an Acute rat

(Figure 3.1A) and a Chronic rat (3.1B).
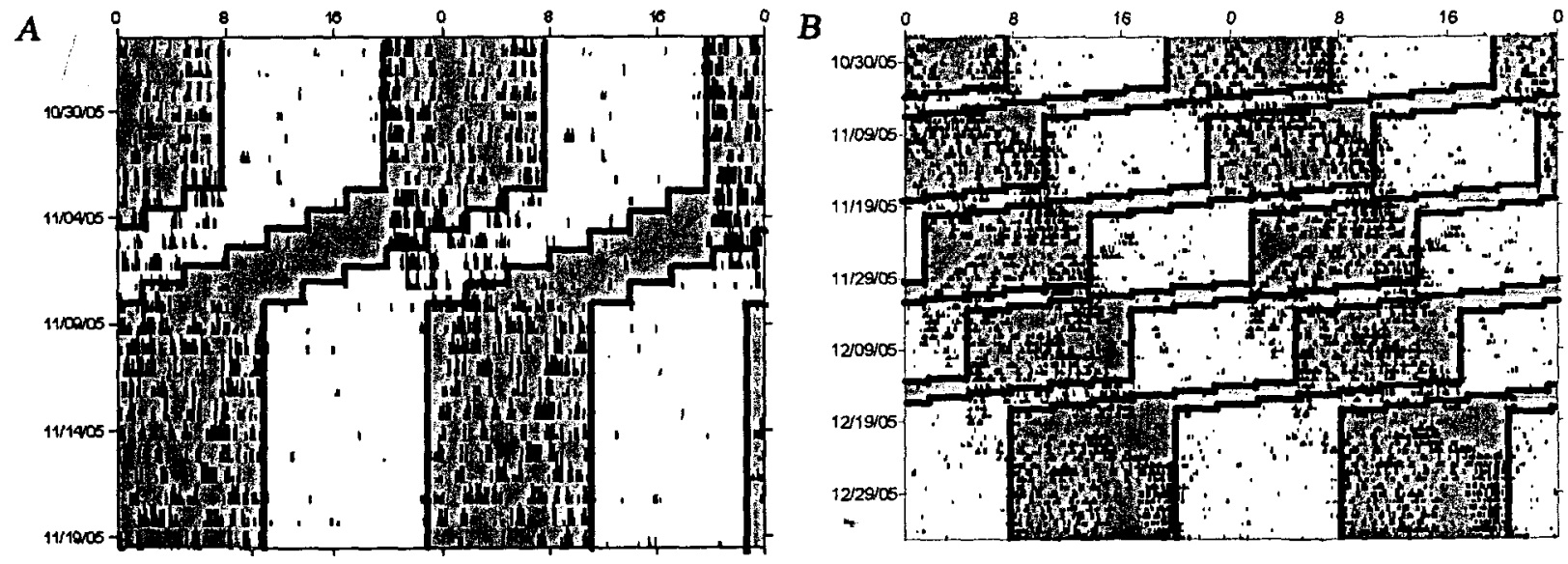

Figure 3.1: Representative actogram from an Acute (A) and a Chronic animal (B). Actograms are double plotted over forty eight hours for clarity. Each horizontal line represents two days, and the vertical bands represent wheel running. The shaded portions refer to when the lights were off. 


\section{Phase angle of entrainment}

During the initial entrainment the rats predictably began running shortly after lights off. This initial entrainment is a good measure of normal circadian rhythms and as such, served as a control for the effects of phase shifting. However, following the first phase shifting session, the phase angle of both groups was significantly delayed, $(-1.55$ $+/-0.25$ hours and $-1.47+/-0.31$ hours, for Acute and Chronic rats respectively). There were no inherent differences between the Acute and Chronic groups before or after the first phase shifting session and both groups showed a significantly delayed phase angle following the initial phase shift. A two-way mixed ANOVA with Group (Acute versus Chronic) as the between subjects variable, and Phase Shift (baseline versus post 1) as the repeated measure found a significant effect of phase shift $[F(1,10)=22.51, p<0.01]$, but no effect of group $[F(1,10)=0.08, p=0.78]$, or interaction $[F(1,10)=0.00, p=0.98]$ (the baseline data from one Acute rat and the post 1 data from one Chronic animal were excluded from the analysis). Tukey post hocs showed the phase angle following the phase shift to be significantly delayed compared to baseline ( $p$ 's<0.05).

To look at the effect of multiple phase shifts and recovery sessions on phase angle, I further examined the activity onsets of the Chronic group. Following the first phase shift the phase angle was delayed, with the activity onset occurring 1.5 hours after lights off (compared to the baseline phase angle of -11.4 minutes). The phase angle was delayed further after the second phase shift (-3.4 hours), and reduced following the third phase shift ( -1.4 hours), where it stabilized for the duration of the experiment (Figure 3.2A). A two-way mixed ANOVA with Group as the between subjects variable and Phase Shift (five levels) as the repeated measure revealed a significant main effect of 
phase shifting $[F(4,24)=5.70, p<0.05]$ (the post 4 data from one rat was excluded). Tukey post hocs revealed that the phase angle following the second phase shift was significantly delayed compared to baseline or post $1\left(p^{\prime} s<0.05\right)$.

\section{Rhythm fragmentation}

Normal rats will confine the majority of their activity to the dark phase of the light cycle, while spending the light phase resting. Therefore, the proportion of activity during the light phase (light activity) is a good measure of rhythm fragmentation. Interestingly, I found a similar pattern of results as observed with the phase angle of entrainment. During the initial entrainment the rats predictably confined their activity to the dark phase with only $4.3+/-0.57 \%$ and $9.4+/-2.99 \%$ light activity for acute and chronic rats respectively. Following the first phase shift, there was an increased proportion of light activity in both groups $(11.5+/-3.55 \%$ and $13.0+/-2.48 \%)$. There were no differences between the Acute and Chronic groups before or after the first phase shifting session. A two-way mixed ANOVA with Group as the between subjects variable, and Phase Shift (two levels) as the repeated measure, revealed no effect of phase shift $[F(1,10)=4.57, p=0.058]$, group $[F(1,10)=1.40, p=0.26]$ or interaction $[F(1,10)=0.54$,

$p=0.48]$ (the baseline data from one Acute rat and the post 1 data from one Chronic animal were excluded from the analysis). However, there was a trend towards increased activity when the lights were turned on in both groups following the phase shift $(p=0.058)$. 


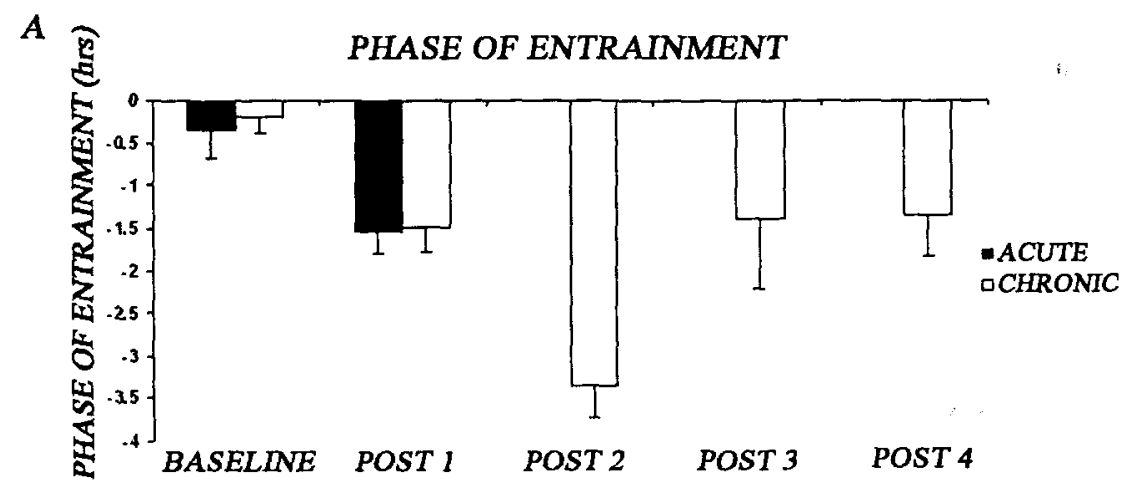

$B$

RHYTHM FRAGMENTATION

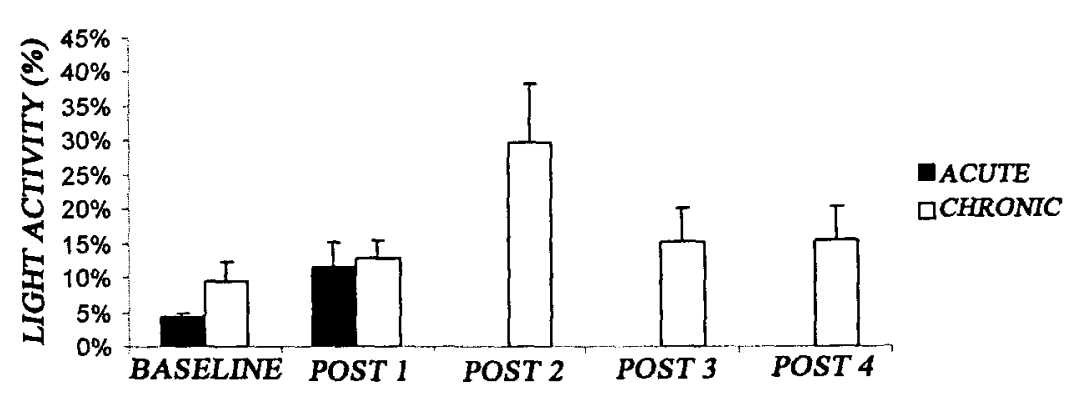

Figure 3.2: Activity onsets and number of wheel rotations were recorded and averaged over the six days prior to phase shifting (baseline), and for the six days following each phase shifting session (post 1-4). The phase angle of entrainment for Acute and Chronic rats shows initial entrainment, then a delayed phase angle peaking after the second phase shift (A). The proportion of total activity occurring during light phase for Acute and Chronic rats shows initial low levels of light activity, followed by a significant peak in light activity following the second phase shift (B). Data are given as means $+/-$ SEM.

To look at the effect of multiple phase shifts and recovery sessions on rhythm fragmentation, I further examined the light activity of the Chronic group. Following the first phase shifting session the proportion of light activity was increased, with $13.0 \%$ of activity occurring during the light phase (compared to the baseline light activity of $9.4 \%$ ). The proportion of light activity was increased further after the second phase shift (29.5\%), and then decreased following the third phase shift (15.2\%), where it stabilized for the duration of the experiment (Figure 3.2B). A two-way mixed ANOVA with Group 
as the between subjects variable and Phase Shift (five levels) as the repeated measure revealed no effect of phase shift $[F(4,16)=2.74, p=0.06]$ (the post 4 data from one rat was excluded), however, there was a trend towards increased activity when the lights were turned on following the second phase shift $(p=0.06)$.

\section{Water maze: Rapid Acquisition Task}

\section{Original location training}

The chronic group had significantly longer latencies to find the platform on all days during original location training (Figure 3.3A). The latency (in seconds) to find the platform was recorded and averaged over the eight daily trials to obtain a single daily latency score for each animal. The daily scores for all four days of original location training were subjected to a two-way mixed ANOVA with Group (Control versus Acute versus Chronic) as the between subjects variable, and Training Day (four levels) as a repeated measure, and revealed a significant effect of group $[F(2,19)=4.70, p<0.05]$ and of day $[F(3,57)=97.74, p<0.01]$, but no interaction $[F(6,57)=1.16, p=0.34]$. By the final day of training, the daily latency score for Chronic rats was more than twice as long as the averaged score for the other two groups $(13.13+/-3.11 \mathrm{sec}$ versus $5.70+/-0.76 \mathrm{sec})$. Tukey post hocs revealed that Chronic rats were impaired compared to the other groups $\left(p^{3} \mathrm{~s}<0.05\right)$. 

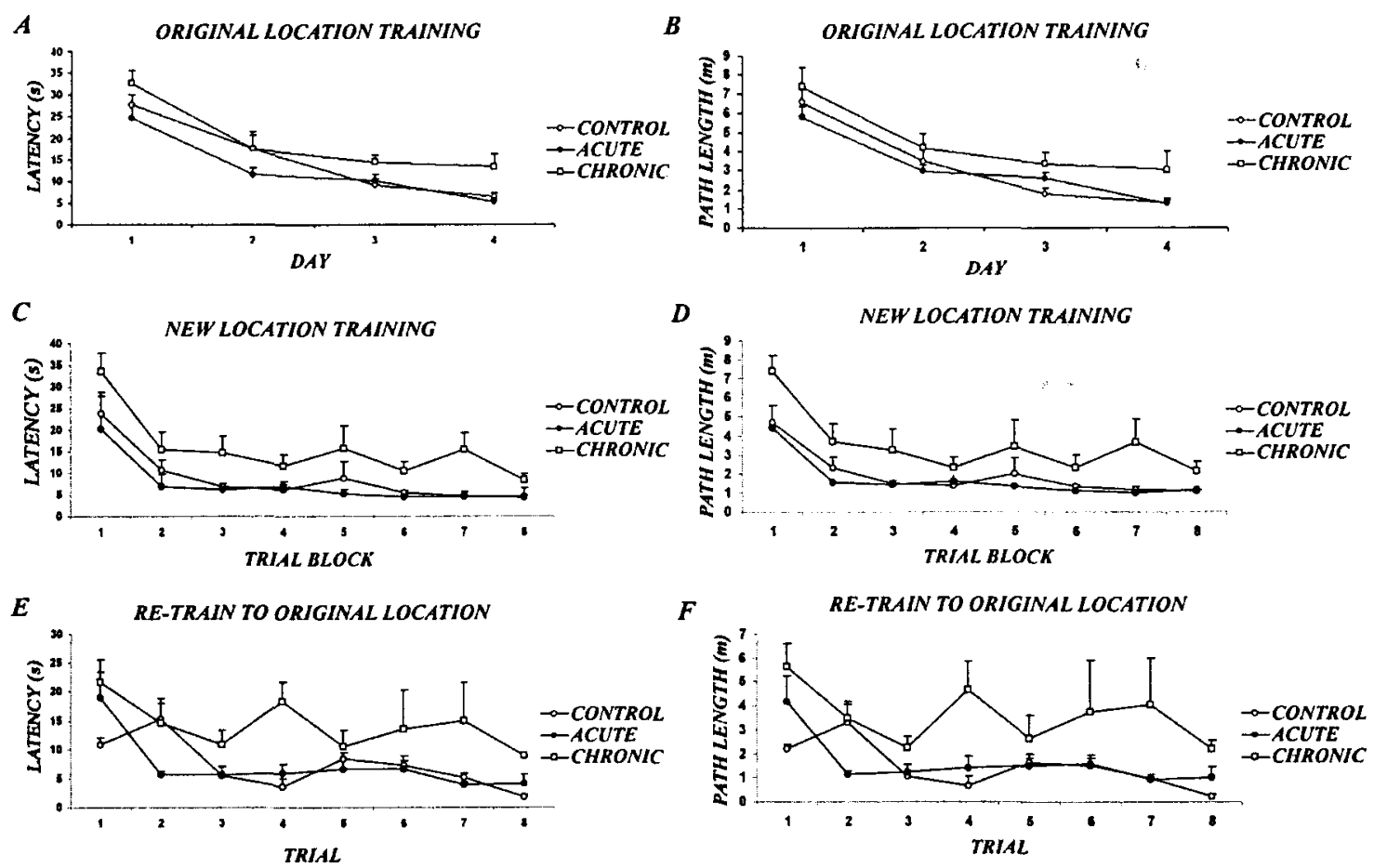

Figure 3.3: Chronic rats are impaired on the spatial water maze task when compared to Acute and Control rats. Both latency to platform and path length data is shown for Chronic, Acute and Control rats on original location training (A,B), new location training $(C, D)$ and re-training to the original platform location $(D, E)$. Data are given as means $+/-$ SEM.

I also measured the length of the path taken to find the hidden platform and found that while the chronically phase shifted rats appeared to have longer path lengths than Acute or Control rats this was not significant (3.3B). A two-way mixed ANOVA with Group as the between subjects variable and Training Day (four levels) as a repeated measure revealed a significant effect of day $[F(3,57)=70.63, p<0.001]$, but no effect of group $[F(2,19)=1.98, p=0.17]$ or interaction $[F(6,57)=0.81, p=0.56]$. My inability to find a difference in path length between groups was likely due to the slightly faster swim speeds of Acute $(0.24+/-0.01 \mathrm{~m} / \mathrm{s})$ and Chronic rats $(0.21+/-0.01 \mathrm{~m} / \mathrm{s})$ compared to Control rats $(0.19+/-0.01 \mathrm{~m} / \mathrm{s})$ during original location training. A one way ANOVA 
with Group as the between subjects variable was used to assess swim speed and revealed a significant effect of group $[F(2,19)=5.46, p<0.05]$. Tukey post hocs revealed that

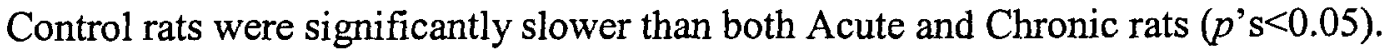

\section{New location training}

The platform was moved to the opposite quadrant of the pool and the latency and path length to find the new platform location was recorded and averaged into eight discrete trial blocks (two trials per block). The Chronic group was impaired in their ability to rapidly acquire a new platform location during this stage of this task when compared to the other groups (Figure 3.3C). By the final trial block, Chronic rats were still taking longer to find the platform $(8.44+/-1.24 \mathrm{sec})$ than the averaged latency of the Control and Acute rats $(4.31+/-1.18 \mathrm{sec})$. A two-way mixed ANOVA with Group as a between subjects variable and Trial Block (eight levels) as a repeated measure, was used to assess the mass training and showed significant effects of group $[F(2,19)=10.88$, $p<0.01]$, and of trial block $[F(7,133)=17.92, p<0.01]$, but no interaction $[F(14,133)=0.49$, $p=0.93]$. Tukey post hocs revealed that Chronic rats were impaired compared to both Control and Acute rats ( $p$ 's $<0.05)$.

The Chronic rats also had significantly longer path lengths than both Acute and Control rats on this stage of the water maze task (3.3D). A two-way mixed ANOVA with Group as the between subjects variable and Trial Block (eight levels) as the repeated measure revealed a significant effect of group $[F(2,19)=6.09, p<0.01]$ and of trial block $[F(7,133)=16.31, p<0.001]$, but no interaction $[F(14,133)=0.66, p=0.80]$. Tukey post hocs showed that Chronic rats had significantly longer path lengths than Acute or Control rats 
$\left(p^{\prime} s<0.05\right)$. A one way ANOVA found no difference in swim speed on this day of training $[F(2,19)=0.87, p=0.43]$.

\section{Re-training to original location}

The platform was returned to the original location (from stage 1) and the latency and path length to find this location was recorded over eight trials on the final day of testing. The results of the competition test are displayed in Figure 3.3E showing that the Chronic rats were still unable to learn the original platform location. By the final trial Chronic rats were taking more than twice as long as the averaged latencies of the Control and Acute rats $(8.91+/-1.36 \mathrm{sec}$ versus $2.97+/-0.92 \mathrm{sec})$. A two-way mixed ANOVA, with Group as the between subjects variable, and Trial (eight levels) as a repeated measure revealed a significant effect of group $[F(2,19)=6.22, p<0.01]$, and of trial $[F(7,133)=5.35, p<0.01]$, but no interaction $[F(14,133)=1.38, p=0.17]$. Tukey post hocs revealed that Chronic rats were impaired compared to both other groups $(p ' s<0.05)$.

Chronic rats also had significantly longer path lengths during reversal training (3.3F). A two-way mixed ANOVA with Group as the between subjects variable and Trial (eight levels) as the repeated measure revealed a significant effect of group $[F(2,19)=4.99, p<0.05]$ and of trial $[F(7,133)=4.26, p<0.001]$, but no interaction $[F(14,133)=1.24, p=0.25]$. Tukey post hocs found that Chronic rats had significantly longer path lengths compared to both other groups $\left(p^{\prime} s<0.05\right)$. I assessed swim speed on this day and found that Chronic rats swam slightly faster than Control rats $(0.24+/-0.01$ $\mathrm{m} / \mathrm{s}$ versus $0.19+/-0.02 \mathrm{~m} / \mathrm{s})$, but were no different from Acute rats $(0.22+/-0.02 \mathrm{~m} / \mathrm{s})$. A one way ANOVA was used to assess swim speed and revealed a significant effect of 
group $[F(2,19)=3.65, p<0.05$. Tukey post hocs showed Chronic rats to be significantly faster than Control rats, but no different from Acute rats $(p$ 's $<0.05)$.

To further assess the competition data, individual swim paths on the first trial were then analyzed to obtain a measure of the animal's memory for the platform locations. The swim paths differed markedly between groups, with the Control and Acute animals swimming first to the new location, and then after failing to find the platform, swimming to the original location (Figure 3.4A, B). In contrast, the Chronic animals appeared to have no clear representation of either location and either swam randomly throughout the pool before finding the platform, or swam to the new or original locations at chance levels (3.4C). In order to quantify this data, I chose to use quadrant entry as a categorical measure of platform memory. Each swim path was classed as new, original, or middle depending on which quadrant the rat first entered upon leaving the starting quadrant. These data were then subjected to a binomial test with the categories set as new or other (combined choice of original and middle), with a 0.33 expected probability of entering the new quadrant. The Control and Acute groups showed a clear preference for the new quadrant as 6 out of 7 Control rats $(p<0.01)$, and 5 out of 7 Acute rats $(p<0.05)$ entered the new quadrant first, whereas only 3 out of 8 Chronic animals entered the new quadrant first $(p=0.524)(3.4 \mathrm{D})$. 

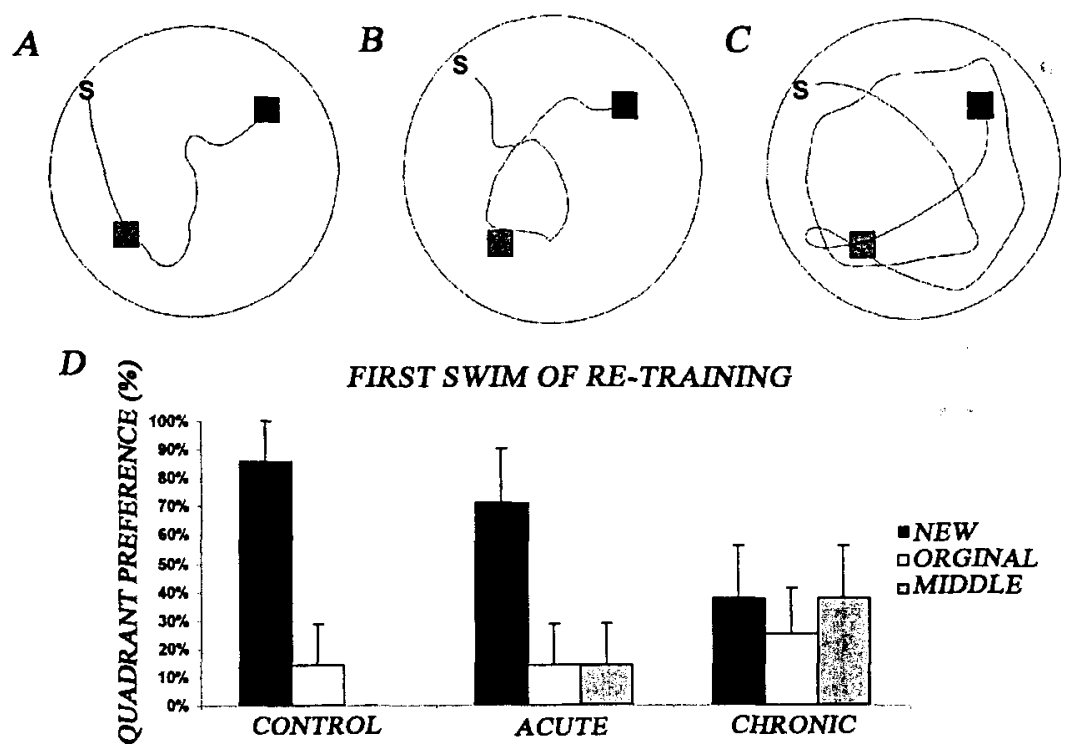

Figure 3.4: Representative swim paths from each group show that Control (A) and Acute (B) rats swam first to the new platform, then headed towards the original platform location and escaped from the water, whilst Chronic (C) rats swam to the new platform at chance levels. The initial swim on the competition day of the spatial water maze was analyzed to determine platform preference (D). Data are given as means +/- SEM.

\section{Fear Conditioning}

\section{Conditioning to tone}

All groups were able to learn the tone-shock association, and showed an increase in freezing behaviour in the presence of the tone. The percentage of freezing during the first four minutes exposed to the tone was calculated and compared to the Context $B$ habituation period. This percentage of freezing for all groups was significantly greater in the presence of the tone $(76.37 \%+/-4.72 \%$ versus $25.10 \%+/-4.84 \%$; Figure $3.5 \mathrm{~A})$. A two-way mixed ANOVA with Group as a between subjects variable, and Tone (habituation versus tone presentation) as a within subjects variable, revealed a significant effect of tone $[F(1,26)=96.84, p<0.01]$, but no group effect $[F(2,26)=1.52, p=0.24]$ or interaction $[F(2,26)=0.22, p=0.81]$. 


\section{Conditioning to context}

All groups were able to learn the context-shock association, and showed an increase in freezing behaviour when returned to Context $\mathrm{A}$ on day three. I measured a percentage freezing for the total time exposed to Context A on day three and compared it to the Context A habituation on day one. The percentage of freezing to context for all groups was significantly greater on day three compared to day one $(74.57 \%+/-3.52 \%$ versus $4.07 \%+/-0.74 \%$; Figure 3.5B). A two-way mixed ANOVA with Group as a between subjects variable, and Context (day one versus day three) as a within subjects variable, revealed a significant effect of context $[F(1,26)=316.61, p<0.01]$, but no group effect $[F(2,26)=1.75, p=0.19]$, or interaction $[F(2,26)=1.87, p=0.17]$.

A

FEAR CONDITIONING TO TONE

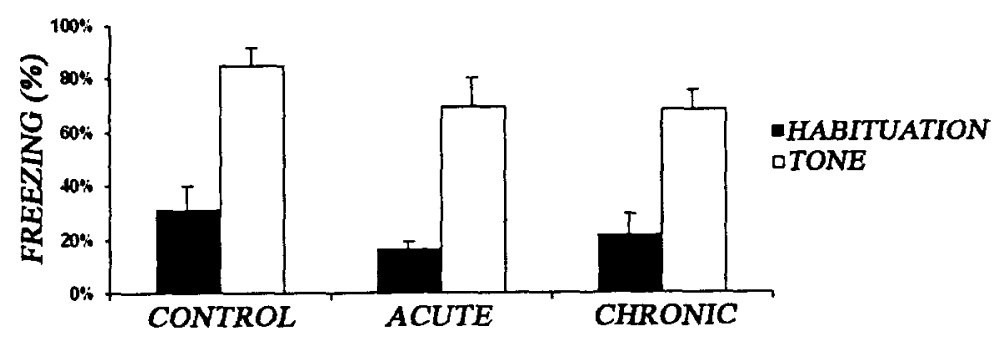

$B$

FEAR CONDITIONING TO CONTEXT

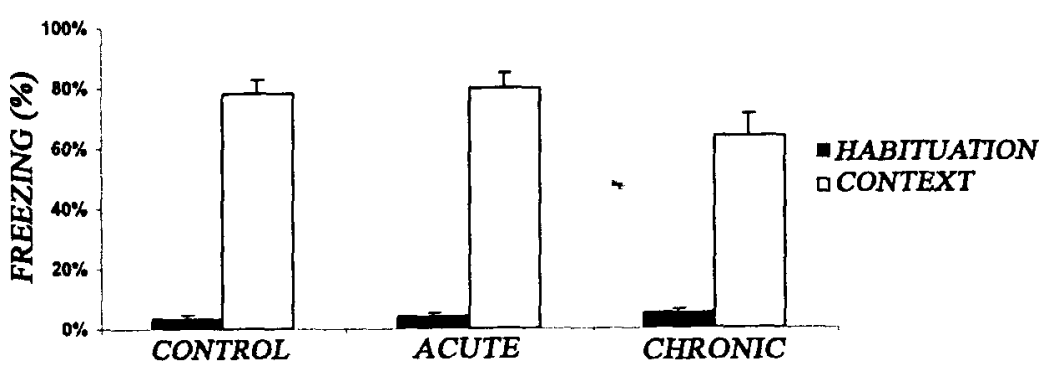

Figure 3.5: Performance on the fear conditioning task. There were no impairments in any of the groups on either fear conditioning to tone (A) or to context (B). Data are given as means $+/$ - SEM. 


\section{Discussion}

Here I show the effects of acute versus chronic circadian disruption on measures of circadian rhythmicity and learning and memory in the rat. Following both acute and chronic phase shifting, the circadian rhythmicity of the animals was disrupted; however this disruption had differential effects on learning and memory dependent on how long the animal was subjected to phase shifting. Chronically phase shifted rats were severely impaired on both acquisition and retention of a platform location in the water maze, while the acutely phase shifted rats performed at control levels. There were some minor differences in swim speed, as the control rats were slightly slower than the acutely phase shifted group during acquisition, and slower than the chronically phase shifted group during the competition test. However, I do not believe these differences affected the overall pattern of results as both phase shifted groups had similar swim speeds overall. No deficit was observed in the fear conditioning task, either to tone or to context, suggesting that the observed deficit was specific to hippocampal memory. These forms of fear conditioning (cued and contextual) are both dependent on the amygdala for successful learning (Fanselow et al., 1999; Ponnusamy et al., 2007). Contextual fear conditioning does have a hippocampal component, but when distinctly different contexts and multiple context-shock pairings are used (as was in the current study) the amygdala is able to successfully form an association without hippocampal involvement (Frankland et al., 1998; Wiltgen et al., 2006).

The ability of these animals to successfully learn the fear conditioning task also demonstrates that the observed impairment on the water maze was not simply a 
performance deficit. The formation of a tone-shock and context-shock association requires motivation to learn, as well as intact motor and sensory skills. The impairment in spatial learning could not be explained by changes in measures of circadian health (phase angle of entrainment and rhythm fragmentation), as these measures were similar for both the unimpaired acute group and the impaired chronic group immediately prior to behavioural testing.

\section{Circadian rhythms and phase shifting}

I found that during the phase shifting procedure, animals dramatically changed their behaviour over the course of multiple phase-shifts. Non-phase shifted rats on a constant light/dark (L:D) schedule reliably begin wheel running at the same time each day, just after lights off (Turek et al., 1995; Antle and Mistlberger, 2005). This pattern of wheel running activity was observed in my animals prior to phase shifting demonstrating entrainment to the existing L:D schedule. However, following phase shifting, the changes in phase angle, and proportion of activity during the light phase in my animals suggests that their ability to maintain circadian rhythmicity had become compromised.

One powerful measure of the presence of the clock and circadian entrainment to an external zeitgeber is to look at an animal's daily onset of activity. Responsiveness to zeitgebers, such as light, decreases with age and is a reliable indicator of an intact healthy clock (Turek et al., 1995). In young healthy animals, the phase angle of entrainment is extremely stable. However, changes in phase angle can be induced by both photic (e.g., phase shifting, light pulse) and non-photic (e.g., novel running wheel, food, receptive mate) stimuli (Janik et al., 1994, Turek et al., 1995). Re-entrainment following photic stimuli is variable and is dependent on the timing of the pulse or the size and direction of 
the phase shift. Tapp and Holloway (1981) reported that within five days, rats can reentrain following a single twelve hour phase shift. Devan et al. (2001) used a similar phase shifting paradigm to the one used in the current study, and found with this more extreme challenge to the circadian system, rats required eighteen days to fully re-entrain. By rapidly repeating phase shifts and recovery sessions, I was able to create a state of chronic circadian disruption where for sixty four days (Chronic group) rats were not given the opportunity to re-entrain. Even twenty six days following the final phase shift, my rats still demonstrated a delayed phase angle (data not shown).

Another reliable sign of circadian health is the presence of distinct activity-rest cycles that correspond with light and dark phases. In nocturnal animals the proportion of total activity occurring during the light phase is a good measure of rhythm fragmentation. Young healthy rats will show little or no activity during the light phase, restricting the majority of their activity to the dark phase. Aged animals, especially those with cognitive impairments, demonstrate fragmented rhythms, with activity spread throughout the twenty four hour period (Antoniadis et al., 2000; Pang et al., 2004). The most common complaints of $\mathrm{AD}$ patients are increased night time awakenings, and day time napping which are indicative of rhythm fragmentation. The connection between rhythm fragmentation and the severity of dementia tends to follow an inverted U curve with the most fragmented rhythms found in moderately demented patients (van Someren et al., 1996). It remains to be seen whether the observed cognitive deficits lead to the disruption of circadian rhythms in these patients, or whether the long-term disruption of rhythms plays a role in the degeneration of memory processes in the elderly. 


\section{Circadian rhythms and cognitive impairment}

The idea that circadian disruption leads to cognitive impairment has been supported by a number of animal studies. Devan et al. (2001) demonstrated a deficit in long-term memory following six days of phase shifting in rats. Animals that were trained on the water maze task during the phase shifting session, and tested seven days later showed impaired retention for the platform location. This appeared to be a deficit in consolidation as opposed to impairment in retrieval as the result of being trained and tested at different circadian times. McDonald et al. (2002) subsequently confirmed this and showed no effect of circadian time on the performance of rats on multiple different memory tasks. In the current study, I have shown that when acutely phase shifted rats, after the same six days of circadian disruption, were trained and tested following ten days of recovery they were not impaired on this task. This suggests that ten days was sufficient time for cognitive processes to recover, even though the animals were not fully reentrained. I further demonstrated that the negative effects of long-term circadian disruption were present in my chronically phase shifted group, resulting in a severe deficit in both acquisition and retention of platform location in the water maze implying specific impairment in hippocampal function, as this group was able to successfully learn a hippocampal independent task (fear conditioning) immediately following water maze testing.

Other studies have shown cognitive impairments in both passive and active avoidance following a single phase shift. Tapp and Holloway (1981) found that this impairment was present both in rats with disrupted circadian rhythms (tested twenty four hours after the phase shift) and in rats that had been allowed to fully re-entrain (tested 
seven days after the phase shift). Fekete et al. (1985) phase shifted rats for varying lengths of time prior to training and found that three days was sufficient for rats to recover from the phase shift on measures of memory and task performance, however, reentrainment of circadian rhythms took longer. It seems that immediately phase shifting post training or a phase shift just prior to testing interferes with memory. The performance of my acutely phase shifted group agrees with these results as their training began ten days post phase shift, more than enough time for memory processes to recover, although re-entrainment was not complete.

The presence of recovery time after an acute challenge to the circadian system is enough to protect the animal from phase shift-induced memory deficits. However, even after ten days of recovery, chronic circadian disruption led to both an acquisition and a retention deficit as observed in chronically phase shifted rats. One weakness of the current study is the difference in housing conditions between the phase shifted groups (individually housed with a wheel) and the non-phase shifted controls (pair housed without a wheel). Voluntary wheel running has been shown to enhance performance on the water maze in both young and aged rats (Adlard et al., 2004; Vaynmann et al., 2004; Alaei et al., 2007). However, I do not believe that using control rats that were housed without a wheel has altered my main finding of a learning and memory deficit following chronic phase shifting. As there were no differences obșerved between control and acute animals, I focused on comparing acute and chronically phase shifted animals directly as both groups were housed with wheels. The memory deficits reported here in the chronic, but not the acute, group suggest that the longer an individual (human or non-human animal) suffers from circadian disruption, the greater the likelihood is that signs of 
dementia or cognitive impairments will develop. Taken together, these studies point towards a link between circadian disruption and impairments in learning and memory tasks.

\section{Possible mechanisms for the negative effects of circadian disruption}

Here I show that chronic, but not acute disruption of circadian rhythms severely impairs both acquisition and retention on a spatial memory task in the rat. Neither group demonstrated complete re-entrainment at the time of behavioural training and testing, and showed similar delays in activity onset, and increased proportion of light activity. Therefore, it is unlikely that differences in circadian phase or rhythm fragmentation are responsible for the impairments observed following chronic phase shifting. Instead, it is likely that long-term exposure to the negative effects of circadian dysfunction can result in impairment. These effects include chronic stress, changes in sleep quality and quantity, and changes in hormone levels such as melatonin, amongst others.

Chronic stress is a commonly proposed mechanism to explain the negative effects of shift work and circadian disnuption. High levels of stress have been reported by individuals employed in shift work and Cho et al. $(2000,2001)$ found a correlation between elevated cortisol levels, increased cognitive impairment and reduced right medial temporal lobe volume in airline crews who had suffered from chronic circadian disruption in the form of continual jet lag for over four years. Increases in stress and levels of stress hormones (cortisol in humans and corticosterone (CORT) in rats) have been linked to cognitive impairments and neuronal damage in both human and nonhuman animals (McEwen and Sapolsky, 1995). Interestingly, elevated cortisol has also been observed in $\mathrm{AD}$ patients and has been hypothesized to play a role in the progression 
of this disorder and aid in the degeneration of the hippocampus (Davis et al., 1986; Lupien et al., 1998).

Luine et al. (1996) examined the effects of stress on learning and memory in rats, using repeated daily sessions of restraint stress and then testing performance on a spatial radial maze task. They demonstrate that seven days of restraint stress had no effect, but fourteen days of restraint stress enhanced performance in the maze. However, twenty one days of stress resulted in a reversible impairment (requiring an eighteen day recovery period) (Luine et al., 1994). McLay et al. (1998) then showed that following eighty days of daily CORT treatment, rats were impaired on the Barnes circular platform task, and this impairment persisted for at least two months. Although no CORT analysis was performed in this study, it is likely that an increase would be observed in my animals due to stresses resulting from the unpredictability of the L:D schedule and the animals' inability to fully re-entrain. Future studies are required to perform a time course of blood sampling to determine CORT levels in my acutely and chronically phase shifted groups. It would be interesting to discover whether a peak in CORT levels will correspond with the peak in delayed phase angle and light activity observed after the second phase shift.

A second possible way chronic circadian disruption could affect cognition is through associated sleep disturbances. Sleep disturbances have been shown to produce cognitive deficits (Rasch and Gais, 2006) and $\mathrm{AD}$ patients in particular tend to show reductions in both REM and non REM sleep, particularly Stage 4 slow wave sleep when memory consolidation is purported to occur (van Someren et al., 1993; Backhaus et al., 2007). If this is the cause of the cognitive impairments observed in this study, similar results might be expected to be noted in both the acutely and the chronically phase shifted 
groups. Cognitive deficits that have been linked to sleep disturbances are associated with a lack of memory consolidation during slow wave and REM sleep, and can appear after as little as one night of disturbed sleep, making it unlikely that sleep loss alone is interfering with hippocampal processing in my study (Rasch and Gais, 2006).

Alternatively, my results could be explained by the theory that over time, changes in the patterning of sleep, due to circadian disruption, could elicit abnormal firing in the hippocampus (Buzsaki, 1989; Stone, 1989) leading to interference with memory consolidation and possible epileptic activity which could further lead to tissue damage and memory loss (Sutherland et al., 1997; McDonald, 2002).

A third consequence of chronic circadian disruption is change in the level of, and secretion patterns of the hormone melatonin. Melatonin is crucial to the proper modulation of the SCN and plays an important anti-oxidant and neuroprotective role in the brain (McArthur et al., 1991; Antle and Mistlberger, 2005). Decreased levels of melatonin have been observed in the aged population, and more so in both pre-clinical and clinical Alzheimer's patients, suggesting a potential link (Liu et al., 1999; Wu et al., 2003). In addition, this hormone has been shown to enhance behavioural recovery and to reduce damage resulting from stroke, Alzheimer's disease and other neurodegenerative diseases in animals (Pei et al., 2003; Feng et al., 2004). Although the loss of melatonin may not directly cause cognitive deficits, with decrèasing level of this hormone, individuals suffering from chronic circadian disruption may become more vulnerable to brain damage leading to memory loss or dementia.

These are only a few of the many contributors to the negative effects of circadian disruption and the most likely explanation will be a combination of these factors. While 
there have been no reports, to my knowledge, describing a causal link between circadian disruption and the appearance of $\mathrm{AD}$ pathology, many animal models of $\mathrm{AD}$ exhibit circadian disruption prior to the appearance of plaques and tangles (Vloeberghs et al., 2001; Pang et al., 2004; Ambree et al., 2006). The many side effects of circadian disruption are stress, decreases in melatonin secretion and sleep deprivation. While sleep deprivation has not been linked to the development of $\mathrm{AD}$ pathology, chronic stress has been found to increase both plaques and tangles in transgenic mouse models of AD (Green et al., 2006; Jeong et al., 2006; Rissman et al., 2007). In addition, melatonin treatment can reduce the deposition of $\beta$-amyloid in transgenic mouse models of $A D$ and protect against oxidative stress (Matsubara et al., 2003; Feng et al., 2004). Further work using animal models should allow me to intensively study these alternative explanations.

\section{Conclusion}

In conclusion, I have demonstrated a significant cognitive impairment in rats following chronic phase shifting. I propose that this deficit is partly the result of chronic stress due to the inherent unpredictability of phase shifting and an inability of the rat to fully re-entrain following each phase shift. However, the stress response is not the sole cause of this impairment. It is likely acting in combination with other negative effects resulting from chronic circadian disruption, such as alterations in sleep patterns and neuronal firing in the hippocampus, and decreases in the neuroprotective hormone melatonin. Future studies will explore these possible mechanisms, and begin to describe the brains' unique response to circadian disruption. I believe that following more extensive phase shifting, this cognitive impairment will gradually increase in type and severity. These deficits may become more global over time, affecting attention, executive 
functioning and habit formation in addition to the spatial memory deficit reported here. The current findings describe a model that will allow me to further study the role chronic circadian disruption has in the development of cognitive deficits in the elderly. 


\section{Chapter 4}

Reduced cholinergic status in hippocampus produces spatial memory deficits when combined with kainic acid induced seizures

Modified from a paper published in Hippocampus, July 23, Epub ahead of print by Laura A. Craig, Nancy S. Hong, Joelle Kopp and Robert J. McDonald in 2008 


\begin{abstract}
Many risk factors have been suggested to increase the likelihood of developing Alzheimer's disease $(A D)$, but apart from rare cases of familial $A D$, no single factor has been shown to be causative on its own. One of these risk factors most commonly associated with Alzheimer's disease is a loss of cholinergic activity in medial temporal structures such as the hippocampus. While the role played by this cholinergic depletion in the development of $\mathrm{AD}$ is unclear, one possibility is that it may increase hippocampal vulnerability to subsequent risk factors. One secondary risk factor that has been associated with $\mathrm{AD}$ is the presence of seizures, which can damage the hippocampus through excitotoxic cell death. To examine the interaction between these two common risk factors on hippocampal function and integrity, I gave rats a focal cholinergic lesion of the medial septum using the specific immunotoxin 192-IgG Saporin, followed two weeks later by a non-convulsive dose of kainic acid. I then assessed rats for seizure severity, hippocampal damage and performance on a spatial memory task. The combination of the two factors resulted in a trend towards increased seizure severity in the cholinergic depleted rats, but more importantly, following cholinergic depletion, rats that displayed non-convulsive seizure behaviour were significantly impaired on a spatial version of the water maze, but not on a cued version of this task suggesting impairment in hippocampal processing. Rats receiving either factor alone had no difficulty learning the spatial memory task. This result could not be explained by seizure severity or gross hippocampal damage, suggesting a more subtle interaction between these two risk factors is responsible for the development of a hippocampal based memory impairment.
\end{abstract}




\section{Introduction}

There are several plausible hypotheses describing causative factors for Alzheimer's disease $(\mathrm{AD})$, but to date, no accurate etiology has been established. A model has been proposed by McDonald (2002) that places risk factors for AD into two groups; passive factors, such as cholinergic depletion, which can increase the vulnerability of hippocampal neurons, and active factors, such as seizures, that have the ability to directly disrupt learning and memory, through the death or dysfunction of hippocampal neurons. The interaction between these various factors should produce specific sub-types of the disorder depending on which factors are present, consequently affecting the diagnosis and future treatment choices for each group of patients.

A significant decline in acetylcholine in the brains of patients with $\mathrm{AD}$ was first reported in 1976 (Davies and Maloney, 1976) and this finding led to the cholinergic hypothesis of $\mathrm{AD}$ (Bartus et al., 1982; Gallagher and Comlombo, 1995; Mesulam, 2004). However, due to the variable success treating patients with cholinergic agonists, the focus of $\mathrm{AD}$ research has shifted away from this theory. However, the consistency of this depletion in $\mathrm{AD}$ patients suggests that, while not causative, levels of acetylcholine may have an important role to play in the development of this disorder, especially in the early stages (Bowen et al., 1992; Perry et al., 1992; Schliebs and Arendt, 2006). In addition to human $A D$ patients, many of the transgenic mice developed to model $A D$, exhibit significantly reduced levels of acetylcholine in the brain, particularly in the hippocampus further strengthening the link between AD and cholinergic depletion (Morley, 2002; Belluci et al., 2006). 
A relationship between $\mathrm{AD}$ and late onset unprovoked seizures, has been suggested in both human and animal studies. A few studies have shown that individuals with epilepsy have a greater risk of developing $\mathrm{AD}$ (Breteler et al., 1995; Thompson and Duncan, 2005) and several clinical studies have found increases in the occurrence of seizures in patients suffering from $\mathrm{AD}$ when compared to non-demented age matched controls (Hauser et al., 1986; Romanelli et al., 1990; Hesdorffer et al., 1996). In addition, several descriptions of transgenic mouse models of $\mathrm{AD}$ have reported increased susceptibility to seizures and lowered seizure thresholds, as well as increased hippocampal damage in response to kainic acid induced seizures (Guo et al., 1999; Del Vecchio et al., 2004). This increased susceptibility may be partially due to decreased levels of acetylcholine in these mice, as enhanced sensitivity to both hippocampal kindling (Kokaia et al., 1996; Ferencz et al., 1997; Ferencz et al., 2001) and generalized seizures (Silveira et al., 2000) have been demonstrated in rats following cholinergic depletion.

Due to the potential relationship between seizures, cholinergic depletion and individuals diagnosed with Alzheimer's, I chose to look at the effects of a non-convulsive seizure induced by a systemic dose of kainic acid in rats following cholinergic depletion, on their ability to successfully learn a spatial version of the water maze. Non-convulsive seizures were used here as a risk factor instead of convulsive seizures due to the finding that animals that suffer convulsive seizures consistently display learning and memory impairment as well as significant hippocampal damage. I predict that following cholinergic depletion, animals will be more susceptible to seizures and that the 
combination of cholinergic depletion and seizures will impair performance in the water maze task.

\section{Materials and Methods}

\section{Animals}

Forty seven male Long Evans hooded rats (300-400g; CCBN breeding colony) were pair housed in standard shoebox housing on a 12 hour light/dark cycle with food and water ad libitum. The rats were divided into four groups; rats receiving cholinergic depletion and kainic acid (AC-kainic; $n=15$ ), rats receiving sham surgery and kainic acid (Kainic; $n=16$ ), rats receiving cholinergic depletion only (AC; $n=8)$ and untreated rats used as controls for behavioural testing (Control; $n=8)$. All animals were cared for and handled in accordance to guidelines set forth by the Canadian Council for Animal Care.

\section{Surgery}

Surgery was performed under Isoflurane anaesthesia $(4 \%$ with $2.0 \mathrm{~L} / \mathrm{min}$ of oxygen for induction and $2 \%$ after surgical plane was established) in a standard stereotaxic apparatus. An incision was made in the scalp and periosteum down the midline. The fascia was pushed to the edges of the skull with a sterile gauze swab and the skin retracted to expose the skull surface. Holes were drilled into the skull using a 1 mm drill bit attached to a high speed drill. Following surgery all animals were given a single dose of buprenorphine (Temgesic; $0.1 \mathrm{mg} / \mathrm{kg}$ subcutaneously) as an analgesic.

\section{Cholinergic depletion}

The specific neurotoxin 192 IgG Saporin (IgG SAP; Chemicon) has been commonly used to induce selective cholinergic lesions in rats depending on the site of infusion. I chose to damage cholinergic neurons in the medial septum and vertical limb of 
the diagonal band of Broca (MS/VDB) which leads to a reduction in levels of hippocampal acetylcholinesterase (AChE). I lowered a 30 gauge cannula, through holes drilled in the skull, into the MS/VDB (AP +0.45; ML +/-0.6; DV -6.6 and $-8.0 \mathrm{~mm}$ from bregma and the skull) and bilaterally infused IgG SAP $[0.15 \mu \mathrm{g} / \mu 1$ dissolved in sterile $1 \mathrm{x}$ phosphate buffer saline (PBS; $\mathrm{pH}$ 7.4)] at a rate of $0.05 \mu 1 / \mathrm{min}$ using a Harvard minipump. Each animal received a total volume of $0.3 \mu \mathrm{l}$ per side at the first injection site (DV -8.0), and $0.2 \mu \mathrm{l}$ per side at the second site (DV -6.6). The cannula was left in place for six and four minutes after the first and second injection respectively to ensure diffusion of the toxin. Sham operated rats underwent the identical procedure, but were infused with $1 \mathrm{x}$ sterile PBS instead of IgG SAP.

\section{Seizures}

Two weeks following surgery AC-kainic and Kainic animals were injected intraperitoneally with $6 \mathrm{mg} / \mathrm{kg}$ i.p. of kainic acid (Ocean Produce International) dissolved in sterile 1x PBS and observed in clear Plexiglas boxes. This dose is considered to be non-convulsive in normal adult rats (Mikulecka et al., 1999; Gobbo and O'Mara, 2004). Video was recorded of the first ninety minutes and scored for seizure severity using a four point ranking scale based on Lothman and Collins (1981): 0-nothing, sleeping; 1immobility, hunched posture, 'staring'; 2-wet dog shakes; 3-mild limbic convulsions, automatisms; 4-severe limbic convulsions, rearing, bilateral clonus, salivation, loss of postural control. The highest seizure stage reached was recorded for each animal.

\section{Behavioural Testing}

Behavioural testing began one week following injection with kainic acid and consisted of a six day version of the water maze task to test for deficits in hippocampal 
processing. A subset of AC-kainic and Kainic rats were also tested on a visible platform version of the water maze to test for general performance deficits.

Water maze: Rapid Acquisition Task

I used a three stage variant of the spatial version of the water maze task (McDonald et al., 2005). A white plastic circular pool $1.4 \mathrm{~m}$ in diameter and $40 \mathrm{~cm}$ deep was filled to within $20 \mathrm{~cm}$ of the top of the wall with water $\left(20-22^{\circ} \mathrm{C}\right)$ made opaque by adding skim milk powder. The invisible platform was $12 \mathrm{~cm}$ in diameter and made of white Plexiglas with holes drilled into the top of it to provide grip for the animals. During training the platform was submerged $2 \mathrm{~cm}$ under water. Extra-maze cues in the training room included five posters of different sizes and orientations mounted on three of the four walls, a computer rack, a door, an animal rack and the experimenter. Four different start points were used [N, S, E, W (not true compass headings)], equally spaced around the pool. The order was randomly selected for each day and each start point was used twice for day one to four and day six. On day five only the three start points farthest from the platform were used (N, E, S) with each start point used five times and the initial start point repeated at the end for a total of 16 trials.

In the first stage (Original location training) rats were trained over four days for eight trials a day, to swim to a fixed, spatial location. Twenty four hours after completion of stage one; the second stage (New location training) began. This stage consisted of new spatial location training. The platform was moved to the opposite quadrant of the pool and animals were trained for sixteen trials within two hours on a single day to swim to the new platform location. Stage two allowed me to ask if rats could learn to go to a new location, during a single intensive training session. The third stage (Re-training to 
original location) occurred twenty four hours after stage two with the platform returned to its original (stage one) location and each animal was given eight trials. This stage allowed me to ask how the massed new location training during stage two affected relearning to the original location. The platform location for each stage is depicted in Figure 2.1.

This version of the water maze was selected due to its sensitivity to hippocampal damage. The first stage allowed me to identify impairment in acquisition and short term memory of an invariant location. If no differences were apparent, the second stage would test the ability of the rat to rapidly form a novel representation in a familiar context. For the third stage, the subjects' are required to re-learn the original location (McDonald et al., 2005).

Data collection: A computer based rat tracker (VP118, HVS Image) was used to collect and analyze data obtained from an overhead video camera. If a rat did not reach the platform 60 seconds after release, the rat was guided manually to the platform (aided placement) and allowed to remain on the platform for 10 seconds before being removed to a holding cage until the next trial. For all three stages the measures of performance were latency to platform and path length to platform. Path length is a sensitive measure that can control for potential differences in swim speed.

\section{Water maze: Visible platform}

The visible platform version of the water maze task was used as a test of general performance, controlling for sensory, motor and motivation deficits. The same pool and room used for the spatial water maze task was used, except that the platform was made visible by attaching a $6 \mathrm{~cm}$ high black block to the top of the hidden platform. This block was raised $2 \mathrm{~cm}$ out of the water, allowing rats to easily see the platform. The visible 
platform task was carried out on day 7 and consisted of two training sessions in a single day. Each session consisted of four trials (lasting 30 seconds) using each start point $(\mathrm{N}$, $\mathrm{W}, \mathrm{E}, \mathrm{S})$ once. The platform was placed in a novel location at the start of each training session to discourage the use of a spatial strategy in the second session.

\section{Histology}

Following behavioural testing all animals were sacrificed with an overdose of Euthanol and transcardially perfused with $1 \mathrm{x}$ PBS followed by $4 \%(w / v)$ paraformaldehyde (PFA). The brains were carefully extracted and placed in $4 \%(\mathrm{w} / \mathrm{v})$ PFA at $4^{\circ} \mathrm{C}$ for 24 hours. Brains were then transferred to a $30 \%(w / v)$ sucrose solution (sucrose was dissolved in $1 \mathrm{x}$ PBS), containing $0.02 \%(\mathrm{w} / \mathrm{v})$ sodium azide at $4^{\circ} \mathrm{C}$ for at least three days. Brains were sectioned on a freezing cryostat at $40 \mu \mathrm{m}$ in a series of five. The first two sections of each series were mounted onto $1 \%(\mathrm{w} / \mathrm{v})$ gelatin $/ 0.2 \%(\mathrm{w} / \mathrm{v})$ chromalum coated slides, while the third, fourth, and fifth series were stored in 1x PBS containing $0.02 \%(\mathrm{w} / \mathrm{v})$ sodium azide at $4^{\circ} \mathrm{C}$ until being processed for immunohistochemistry. The fifth series was not used in this experiment and was stored for future analyses.

\section{Cresyl violet staining and volumetrics}

The first series was stained with Cresyl violet and analyzed to determine the extent of damage to the hippocampus. Working in a rostral-caudal direction, coronal sections were examined and at the first sign of hippocampal damage pictures were taken under a 10x objective to document the extent of the lesion. I continued to take pictures of every fifth section leaving approximately $200 \mu \mathrm{m}$ between sections sampled to ensure no cell, or hippocampal region was counted twice. After all documentation of the damage 
for each subject in all of the sections was obtained (usually three to four sections), volumetric analysis was completed manually using an AxioVision microscope with associated Axio Imager analysis software (version 4.3). Briefly, the extent of damage in the CA fields and dentate gyrus of the hippocampus were traced and a calculation of the volume of the lesion within the traced areas was obtained. The values from the traced areas were calculated by the computer software in millimetres squared. The total volume of damage for each animal was calculated and used to obtain an average for each group.

\section{AChE staining}

The second series was stained for $\mathrm{AChE}$ to get a qualitative image of cholinergic depletion in the hippocampus. I treated the slides for 30 minutes with $6.24 \mathrm{mg}$ tetraisopropylpyrophosphoramide dissolved in $200 \mathrm{~mL}$ of distilled water, then rinsed the slides twice with distilled water and incubated them on a shaker table for four hours in a reaction mixture containing $5.72 \mathrm{~g}$ Trizma maleate, $2.04 \mathrm{~g}$ Trizma base, $100 \mathrm{mg}$ acetylthiocholine iodide, $294 \mathrm{mg}$ dihydrous tri-sodium citrate, $150 \mathrm{mg}$ anhydrous cupric sulphate and $32.8 \mathrm{mg}$ potassium ferricyanide dissolved in $200 \mathrm{~mL}$ distilled water (all chemicals were obtained from Sigma). Slides were rinsed twice with distilled water, and left to dry overnight, then cleared with HemoDe (Sigma) and coverslipped with Permount (Sigma) the next day.

\section{Immunohistochemistry}

The third and fourth series were processed for immunohistochemistry to determine the completeness and specificity of the cholinergic depletion. An antibody raised against parvalbumin was used to label $\gamma$-aminobutyric acid (GABA)-ergic neurons, and one against choline acetyltransferase (ChAT) was used to label cholinergic neurons. 
Free floating sections were rinsed in $1 \times$ PBS (pH 7.4), and then placed in a $3 \%(v / v)$ $\mathrm{H}_{2} \mathrm{O}_{2}$ solution for four minutes to block endogenous peroxidases. After three washes in $1 \mathrm{x}$ PBS, sections were blocked for 30 minutes in $15 \%(\mathrm{v} / \mathrm{v})$ normal horse serum (for antiparvalbumin; Vector Labs) or 15\% (v/v) normal rabbit serum (for anti-ChAT; Vector Labs). Sections were incubated overnight in primary antibody [anti-ChAT polyclonal, 1:1000 (Chemicon) or anti-parvalbumin monoclonal, 1:5000 (Sigma)] diluted in 1x PBS with $7.5 \%(v / v)$ serum (horse for anti-parvalbumin; rabbit for anti-ChAT). Sections were then washed three times in 1x PBS and incubated for 30 minutes in a biotinylated secondary antibody (ABC Elite Kit, Vector Labs), washed three times in 1x PBS, incubated for 30 minutes with $\mathrm{AB}$ reagent ( $\mathrm{ABC}$ Elite Kits, Vector Labs), washed three times in 1x PBS then developed with 3,3'-diaminobenzidine (DAB) substrate according to the manufacturer's instructions (Vector Labs). Sections were then mounted onto $1 \%$ $(\mathrm{w} / \mathrm{v})$ gelatin/0.2\% (w/v) chromalum coated slides, air dried overnight, then dehydrated in alcohol, cleared in HemoDe (Sigma) and coverslipped with Permount (Sigma).

Cell counts: Under light microscopy (AxioVision) ChAT positive neurons were counted in both hemispheres of the MS/VDB. I chose two sections to quantify; the first, at the site of injection and the second, in a section $200 \mu \mathrm{m}$ caudal to the injection site. This quantification was not used as an accurate count of MS/VDB neurons, but instead to estimate the completeness of the cholinergic depletion. Each hemisphere was counted separately to confirm lesion symmetry, after which counts for both hemispheres were then totaled for a representative cell count over the two sections. 


\section{Results}

Two rats assigned to the AC-kainic group were removed from this experiment due to incomplete cholinergic lesions ( $<40 \%$ depletion) leaving a total of 13 rats in this group.

\section{Seizures}

Following the injection of kainic acid (6 mg/kg i.p.), most rats had mild nonconvulsive (stage 1 or 2 ) seizures consisting of freezing, and wet dog shakes which are common observations during kainic acid induced seizures in rats. A few animals in each group (4 out of 16 Kainic (25\%) and 6 out of 13 AC-kainic rats (46.2\%) displayed convulsive (stage 4) seizures characterized by bilateral forelimb clonus and rearing, followed by loss of postural control and hindlimb clonus. One AC-kainic and one Kainic rat died following two hours of continual convulsions and were not used for further analysis. Animals were divided by seizure type (convulsive or non-convulsive) and I found that although it appeared that rats were more likely to have a convulsive seizure in response to kainic acid following cholinergic depletion, compared to those having a sham surgery, this was not significant. An independent samples t-test was performed and found no difference in seizure severity between groups $[t(27)=1.21, p=0.236$; Figure 4.1].

\section{Water maze: Rapid Acquisition Task}

Because of the small number of surviving rats that had convulsive seizures and to control for the effect of seizure severity on behavioural testing, I decided to exclude all rats that had a convulsive seizure from subsequent analysis. Convulsive seizures induced by systemic kainic acid have been previously shown to cause hippocampal damage as well as cognitive and performance deficits on hippocampal memory tasks (Milgram et 
al., 1988; Gayoso et al., 1994). The following histological and behavioural analyses included seven AC-kainic rats, twelve Kainic rats, eight AC rats and eight Control rats.

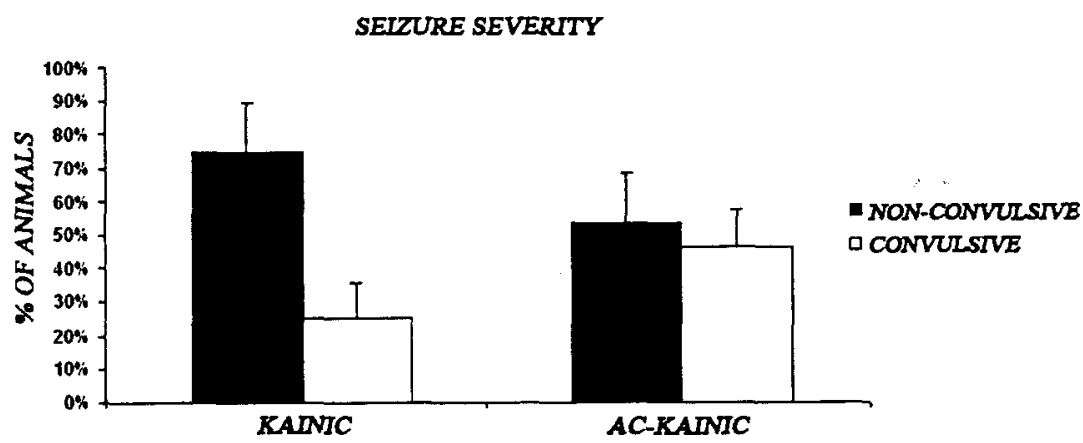

Figure 4.1: Following injection with a non-convulsive dose of kainic acid, $46.2 \%$ of ACkainic rats and $25.0 \%$ of Kainic rats had fully convulsive seizures. The remainder of the rats displayed behavioural signs indicative of non-convulsive seizures. Though there was a trend towards greater seizure severity in the AC-kainic group, there was no significant difference between groups. Data are given as means $+/$ - SEM.

\section{Original location training}

The results of the original platform location training are depicted in Figure 4.2A. The AC-kainic group was significantly impaired on all days in their ability to learn the platform location during original location training when compared to the $\mathrm{AC}$, Kainic and Control groups. The latency (in seconds) to find the platform was recorded and averaged over the eight daily trials to obtain a single daily latency score for each animal. The daily scores for all four days of acquisition were subjected to a two-way mixed analysis of variance (ANOVA) with Group (AC-kainic versus AC versus Kainic versus Control) as the between subjects variable, and Training Day (four levels) as a repeated measure, and revealed a significant effect of group $[F(3,31)=17.53, p<0.01]$ and of day $[F(3,93)=103.96, p<0.01]$, but no interaction $[F(9,93)=1.89, p=0.06]$. By the final day of training, the daily latency score for AC-kainic rats was almost twice as long as the 
averaged score for the other three groups $(15.23+/-3.25$ seconds versus $6.79+/-1.00$ seconds). Tukey post hocs revealed that AC-kainic rats were impaired compared to all other groups $(p ' s<0.01)$.

I also measured the length of the path taken to find the hidden platform and found that AC-kainic rats had significantly longer path lengths than Kainic, AC or Control rats (4.2B). A two-way mixed ANOVA with Group as the between subjects variable and Training Day (four levels) as a repeated measure revealed a significant effect of group $[F(3,31)=8.80, p<0.01]$ and day $[F(3,93)=89.37, p<0.001]$, but no interaction $[F(9,93)=1.06, p=0.40]$. Tukey post hocs revealed that AC-kainic rats had significantly longer path lengths than any of the other groups $\left(p^{\prime} s<0.05\right)$. A one way ANOVA found no difference in swim speed on this stage of training $[F(3,31)=1.00, p=0.40]$.

\section{New location training}

The platform was moved to the opposite quadrant of the pool and the latency (in seconds) to find the new platform location was recorded and averaged into eight discrete trial blocks (two trials per block). The AC-kainic group was impaired in their ability to rapidly acquire a new platform location on this stage of this task when compared to all other groups (Figure 4.2C). By the final trial block, AC-kainic rats were still taking longer to find the platform $(15.75+/-7.65$ seconds $)$ than the averaged latency of the other three groups (4.38 +/- 0.44 seconds). A two-way mixed ANOVA with Group as a between subject variable and Trial Block (eight levels) as a repeated measure, was used to assess new location training and found a significant effect of group $[F(3,31)=7.54$, $p<0.01]$ and of trial block $[F(7,217)=45.12, p<0.01]$, but no interaction $[F(21,217)=1.32$, 
$p=0.17]$. Tukey post hocs revealed that AC-kainic rats were impaired compared to all other groups $(p$ 's $<0.01)$.

$\boldsymbol{A}$

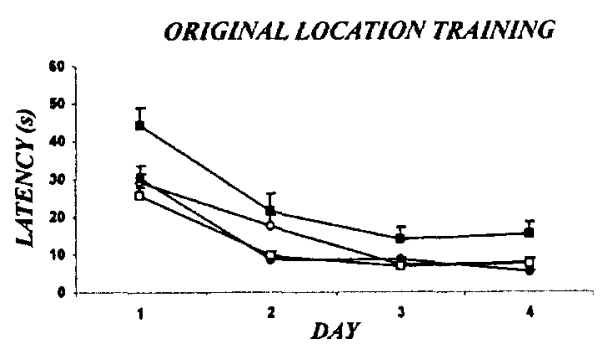

C

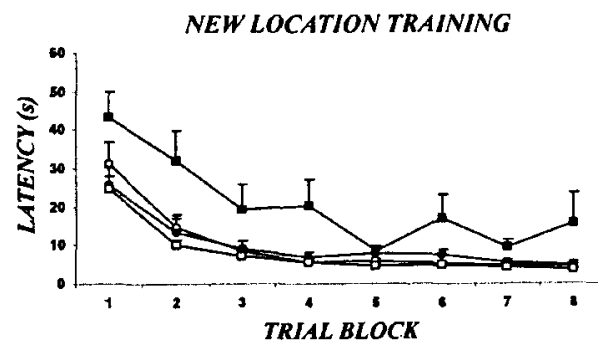

E

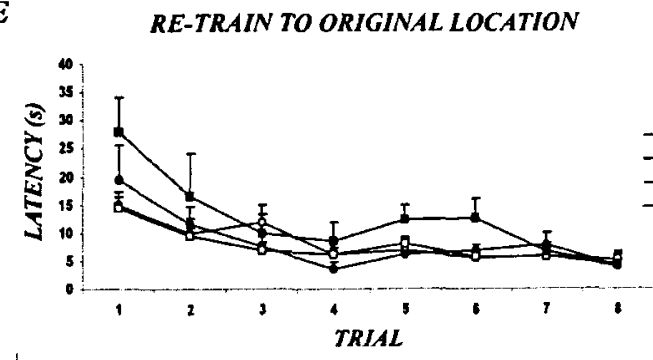

B

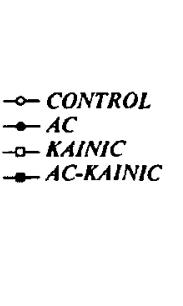

D
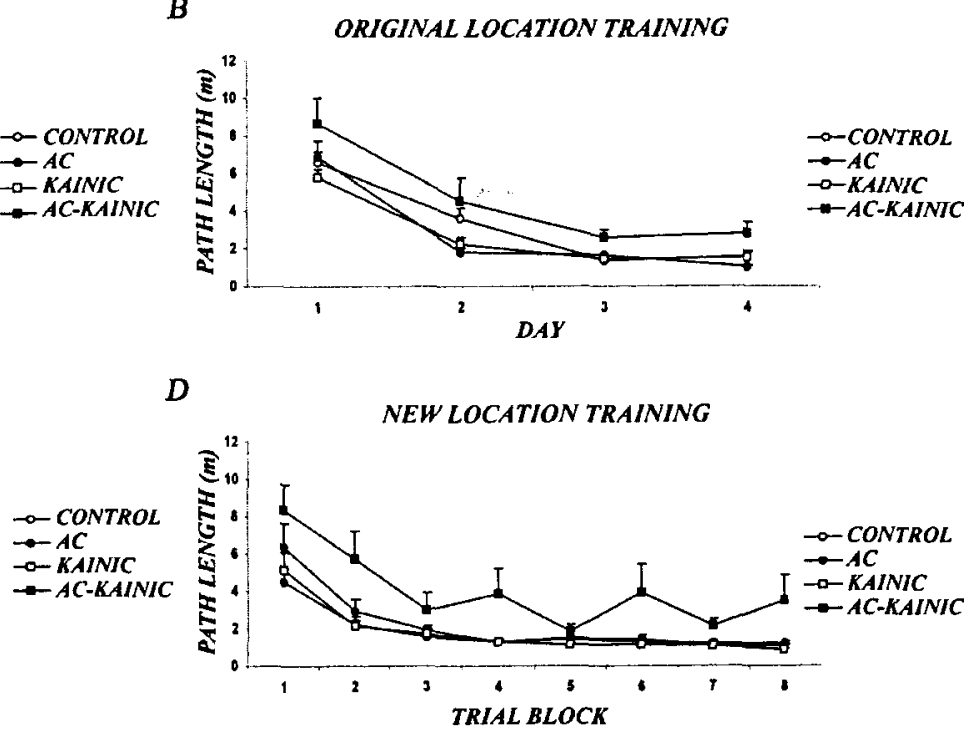

F

RE-TRAIN TO ORIGINAL LOCATION
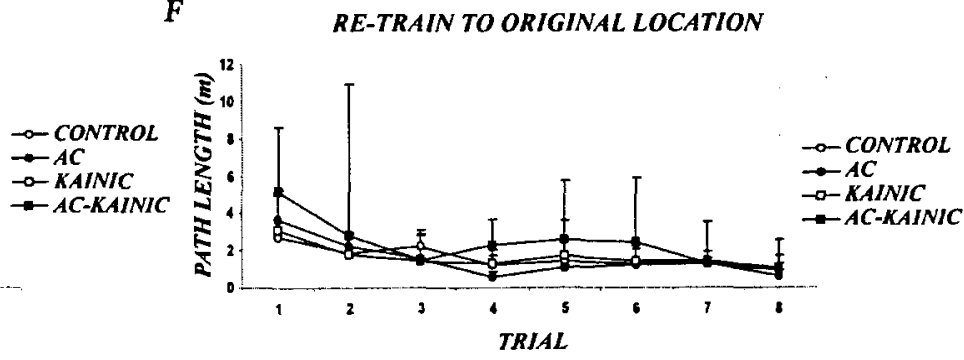

Figure 4.2: AC-kainic rats have longer latencies and path lengths to find the hidden platform on all stages of the spatial water maze task. AC-kainic rats are significantly impaired compared to $\mathrm{AC}, \mathrm{Kainic}$ and Control rats during original location training ( $\mathrm{A}$, $B)$, new location training $(C, D)$ and re-training to the original location $(E, F)$. Data are given as means $+/$ - SEM.

The AC-kainic rats also had significantly longer path lengths than Kainic, AC and Control rats on this stage of the water maze task (4.2D). A two-way mixed ANOVA with Group as the between subjects variable and Trial Block (eight levels) as the repeated measure revealed a significant effect of group $[F(3,31)=9.77, p<0.001]$, of trial block $[F(7,217)=32.07, p<0.001]$, but no interaction $[F(21,217)=1.01, p=0.46]$. Tukey post hocs 


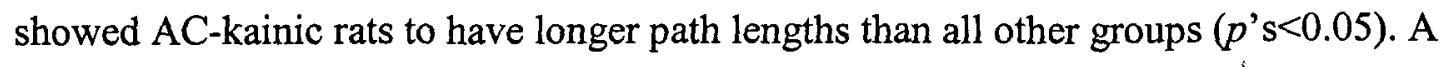
one way ANOVA found no difference in swim speed on this day of training $[F(3,31)=2.43, p=0.08]$.

Re-training to original location

The platform was returned to the original location (from stage one) and the latency and path length to find this location was recorded over eight trials on the final day of testing. The results of the re-training to original location trials are displayed in Figure 4.2E showing that the AC-kainic rats were impaired in their ability to re-learn the original platform location. A two-way mixed ANOVA, with Group as the between subjects variable, and Trial (eight levels) as a repeated measure revealed a significant effect of group $[F(3,30)=3.04, p<0.05]$, and of trial $[F(7,210)=14.27, p<0.01]$, but no interaction $[F(21,210)=0.97, p=0.50]$. Holm-Bonferroni post hocs revealed that AC-kainic rats were impaired compared to all other groups ( $\left.p^{\prime} s<0.05\right)$.

There was a trend towards longer path lengths in the AC-kainic rats during retraining but this did not reach significance $(4.2 \mathrm{~F})$. A two-way mixed ANOVA with Group as the between subjects variable and Trial (eight levels) as the repeated measure revealed a significant effect of trial $[F(7,210)=11.02, p<0.001]$, a nearly significant effect of group $[F(3,30)=2.83, p=0.06]$, but no interaction $[F(21,210)=0.85, p=0.65]$. I assessed swim speed on this day and found no difference in swim speed on this day of training $[F(3,31)=1.66, p=0.20]$.

To further assess the competition data, individual swim paths on the first trial were analyzed to obtain a measure of the animal's memory for the platform locations. The swim paths differed markedly between groups, with the Control, AC and Kainic 
animals swimming first to the new location, and then after failing to find the platform, swimming to the original location (Figure 4.3A-C). In contrast, the AC-kainic animals appeared to have no clear preference, and swam to either the original or the new location at chance levels (4.3D). In order to quantify these data, I chose to use quadrant entry as a categorical measure of platform memory. Each swim path was classed as new, original, or middle depending on which quadrant the rat first entered upon leaving the starting quadrant. These data were then subjected to a binomial test with the categories set as new or other (combined choice of original and middle), with a 0.33 expected probability of entering the new quadrant. The Control, $\mathrm{AC}$ and Kainic groups showed a clear preference for the new quadrant as seven out of eight Control, seven out of eight AC, and ten out of twelve Kainic rats $(p<0.05)$ entered the new quadrant first. The AC-kainic animals appear to have no clear preference and swim to either the new or original quadrant at chance levels as only four out of seven rats entered the new quadrant first $(p=0.17 ; 4 \mathrm{E})$.

The performance of rats on this hippocampal dependent task following cholinergic depletion combined with non-convulsive seizures was significantly compromised. All three stages of this task were affected. AC-kainic rats were delayed during original location training and in their ability to learn a new location in a single day. An analysis of swim paths on the final day of testing revealed no clear memory for either of the locations, in contrast to the performance of Control, AC and Kainic groups that showed a clear preference for the most recently learnt location. 

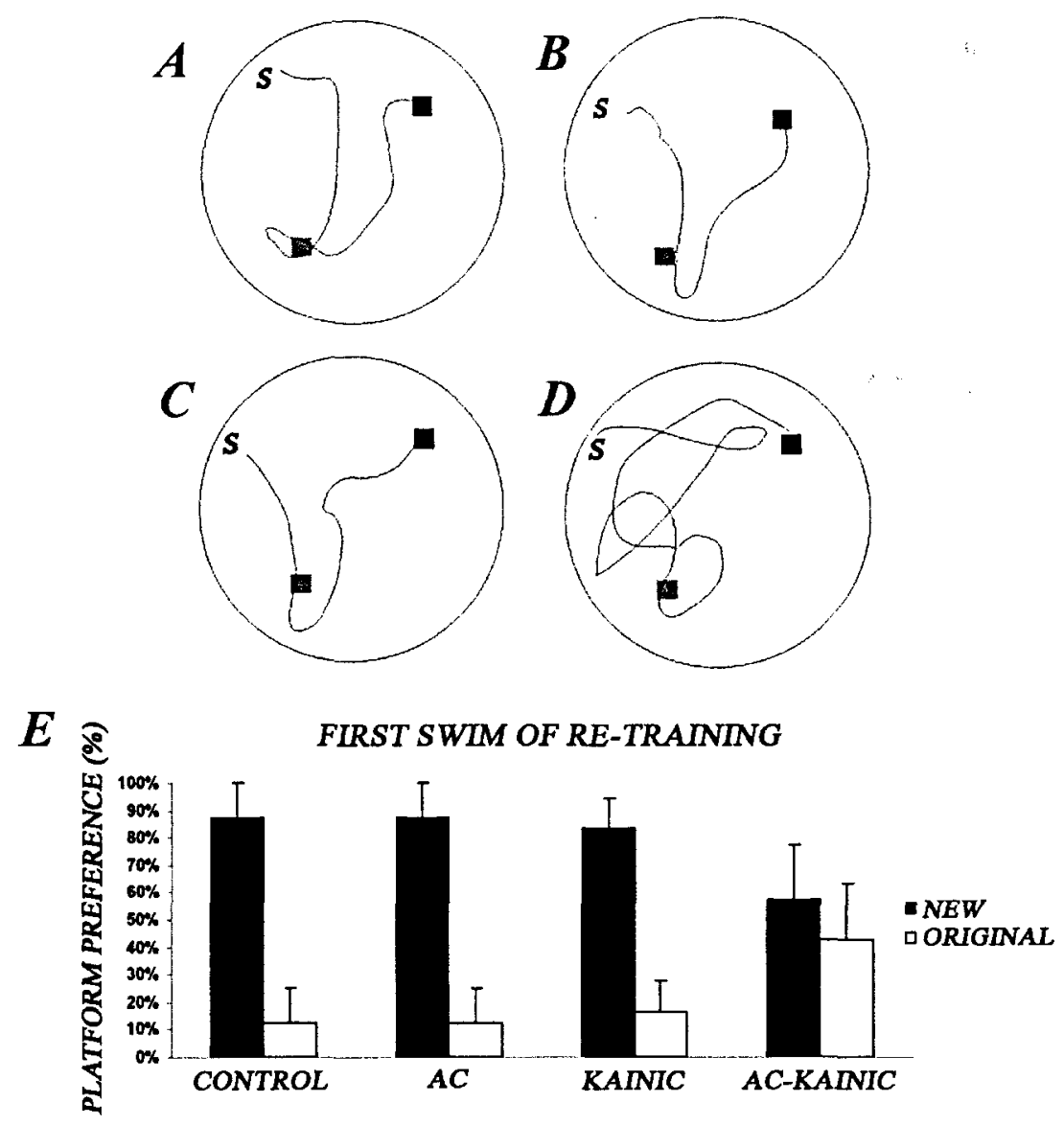

Figure 4.3: Representative swim paths from each group show that Control (A), AC (B) and Kainic $(C)$ rats swam first to the new platform, then headed towards the original platform location and escaped from the water, while AC-kainic rats swam to either location at chance levels (D). The initial swim on the competition day of the spatial water maze was analyzed to determine platform preference (E). Data are given as means $+/-$ SEM.

\section{Water maze: Visible Platform Task}

I tested a subset of AC-kainic and Kainic rats on the visible platform task to determine whether changes in motivation or sensory and motor deficits were responsible for the observed impairment in the spatial water maze. No difference between groups was observed (Figure 4.4) and the latencies were comparable to those previously reported for $\mathrm{AC}$ and Control rats tested on a similar paradigm (Craig et al. submitted). A two-way 
mixed ANOVA with Group (AC-kainic versus Kainic) as the between subjects variable and Session (two levels) as the repeated measure revealed a significant effect of session $[F(1,9)=9.63, p<0.05]$, but no effect of group $[F(1,9)=0.54, p=0.48]$ or interaction $[F(1,9)=1.44, p=0.26]$. Follow up Tukey post hocs showed both groups had significantly shorter latencies during the second training session $\left(p^{\prime} \mathbf{s}<0.05\right)$.

VISIBLE PLATFORM TRAINING

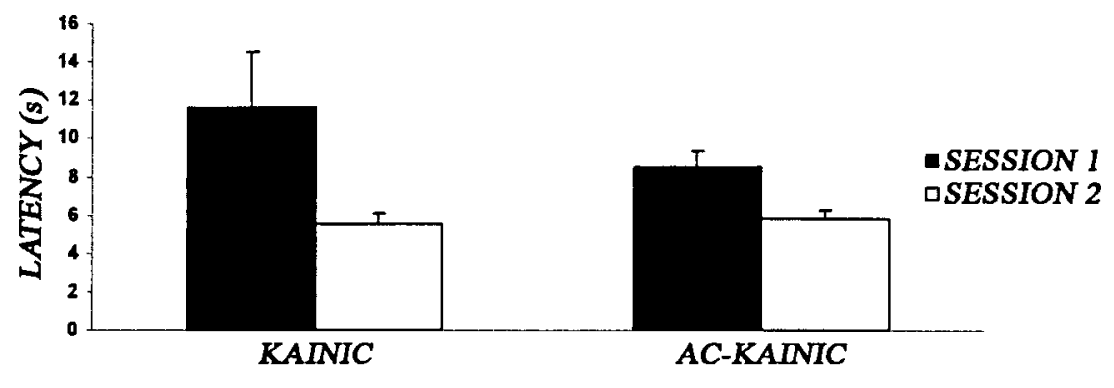

Figure 4.4: There were no differences between Kainic and AC-kainic rats on a visible platform version of the water maze, suggesting the observed impairment in the spatial water maze was due to a hippocampal specific deficit. Data are given as means +/- SEM.

\section{Histology}

Quantification of the cholinergic lesion in the medial septum

I confirmed the completeness and specificity of the cholinergic lesion in the medial septum of both $\mathrm{AC}$ and $\mathrm{AC}$-kainic groups through $\mathrm{AChE}$ staining and immunohistochemistry for $\mathrm{ChAT}$ and parvalbumin. Following cholinergic depletion, animals had very few ChAT positive cholinergic neurons (Figure 4.5A-C) combined with intact parvalbumin labeled GABA-ergic neurons (4.5D-F) confirming the specificity of the cholinergic lesion. The $\mathrm{AChE}$ staining clearly showed a depletion of cholinergic activity in the hippocampus of all lesioned animals compared to the sham operated group 
(4.5G-I).
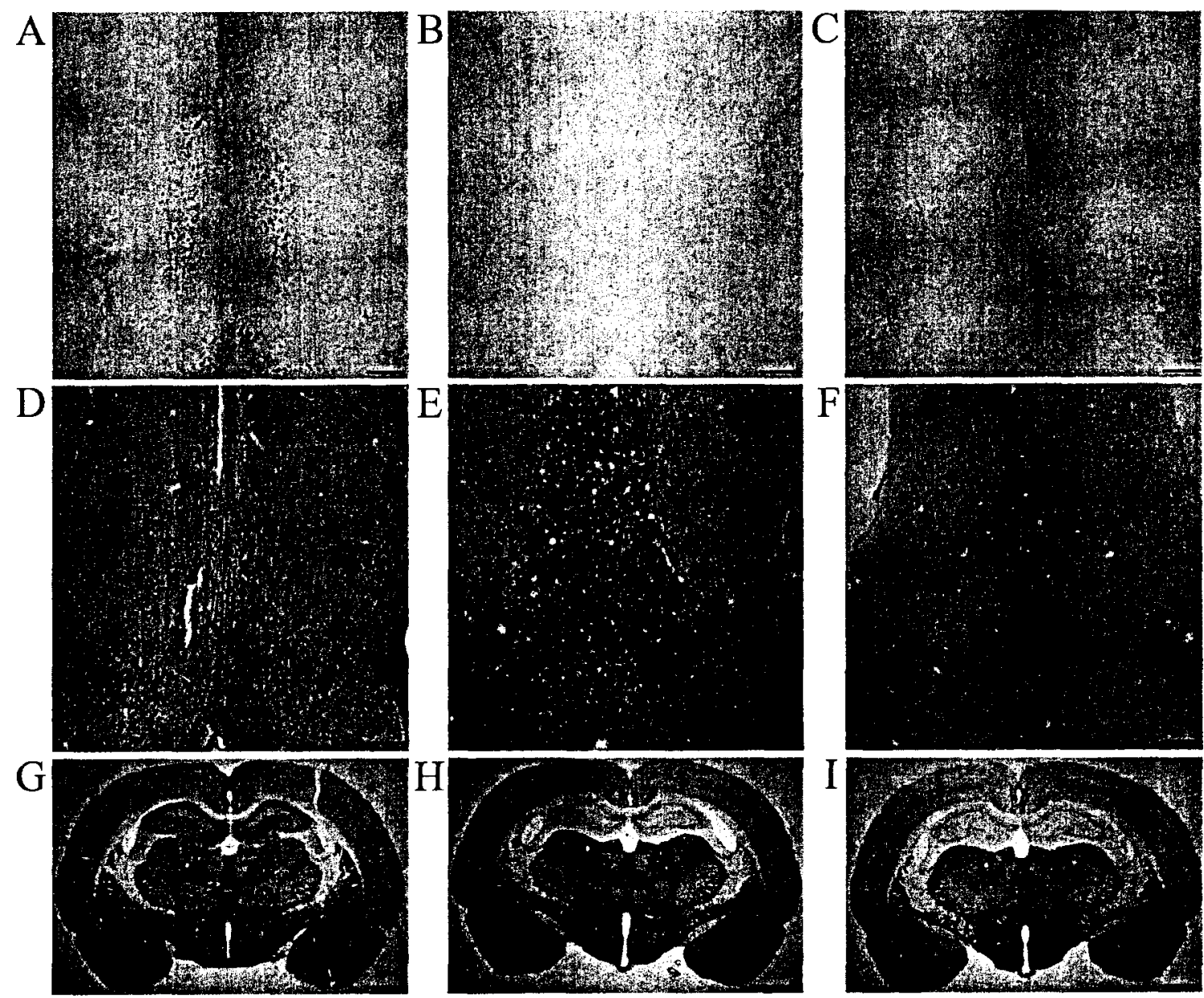

Figure 4.5: Photomicrographs of anti-ChAT labeling in the MS/vDB from a Kainic rat (A), an AC rat (B) and an AC-kainic rat (C) confirm the near total loss of cholinergic neurons in this area following infusion of IgG SAP. Anti-parvalbumin labeling in the MS/vDB from a Kainic rat (D), an AC rat (E) and an AC-kainic rat (F) confirm the specificity of this lesion to cholinergic neurons, while sparing GABA-ergic neurons. The normal $\mathrm{AChE}$ staining apparent in the dorsal hippocampus of a Kainic rat $(\mathrm{G})$ is dramatically reduced following cholinergic depletion in the hippocampus of an $\mathrm{AC}$ rat (H) and an AC-kainic rat (I). Scale bars, $0.1 \mathrm{~mm}(\mathrm{~A}-\mathrm{F})$ and $1.0 \mathrm{~mm}$ (G-I).

I quantified the extent of the cholinergic lesion by comparing the ChAT positive

cell counts between groups. To control for any possible effects of systemic kainic acid on the number of cholinergic neurons in the medial septum, I used the ChAT positive cell counts from the Kainic group to determine a percent depletion for the AC-kainic group and used the cell counts from the Control group to determine the percent depletion for the 
AC group. I found $90.11+/-7.26 \%$ depletion in the AC-kainic group and $96.81+/-$ $1.75 \%$ depletion in the $\mathrm{AC}$ group (Two AC-kainic rats had incomplete lesions with less then $40 \%$ depletion and were removed from the study). There were significantly fewer cholinergic neurons in the $\mathrm{AC}$ and $\mathrm{AC}-$ kainic groups compared to the Kainic and Control groups (Table 4.1). A one way ANOVA revealed a significant effect of group $[F(3,30)=72.09, p<0.01]$. Tukey post hocs showed that there were no differences between the extent of the depletion in $\mathrm{AC}$ and $\mathrm{AC}-\mathrm{kainic}$ groups, and both groups had significantly fewer ChAT positive cells than either the Kainic or Control groups $(p ' s<0.05 ;$ Figure 4.6)

Table 4.1: The number of cholinergic neurons in the medial septum totaled over two sections in both lesioned and sham operated rats.

\begin{tabular}{lccc}
\hline Group & \multicolumn{3}{l}{ Number of ChAT positive neurons } \\
\cline { 2 - 4 } & Section 1 & Section 2 & Total \\
\hline Control & $125.00+/-12.29$ & $129.71+/-19.06$ & $254.71+/-25.99$ \\
Kainic & $160.17+/-14.34$ & $169.17+/-12.60$ & $329.33+/-18.03$ \\
AC & $8.13+/-4.45$ & $0.00+/-0.00$ & $8.13+/-4.45$ \\
AC-kainic & $10.29+/-4.55$ & $22.29+/-21.30$ & $32.57+/-23.91$
\end{tabular}

Data are given as means $+/$ - SEM. Control rats are from a separate experiment and included only as a general comparison. 


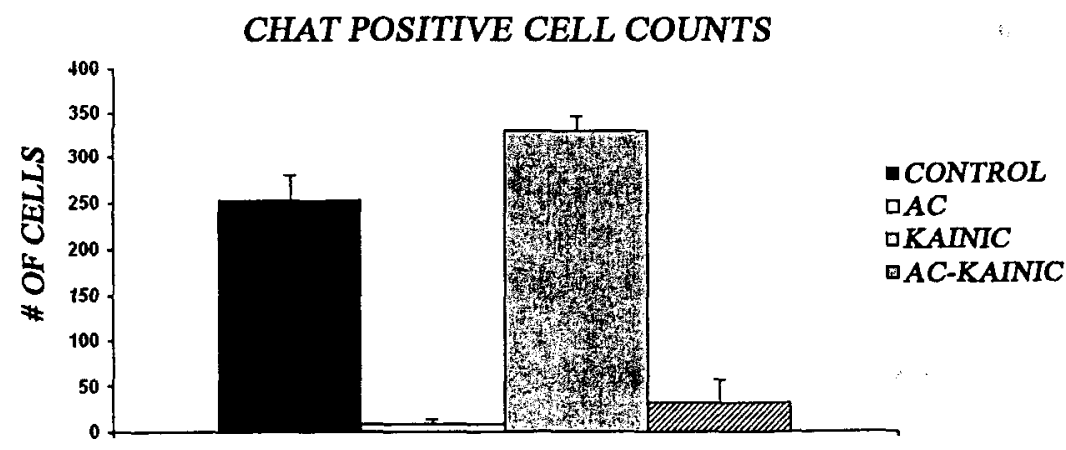

Figure 4.6: Choline acetyltransferase (ChAT) positive cell counts show a clear depletion in AC (97\%) and AC-kainic (89\%) rats compare to Kainic and Control rats. Control rats are from a separate experiment and included only as a general comparison. Data are given as means +/- SEM.

Lesioned animals had areas of necrotic cell death in the MS/VDB surrounding the injection site. This non-specific damage has been reported previously (Heckers et al., 1994; Frick et al., 2004) and work in our laboratory has shown no correlation between lesion size (due to focal necrosis following infusion of the toxin) and water maze performance (data not shown).

Analysis of hippocampal damage

The hippocampus was visually inspected under light microscopy in the Cresyl violet stained sections, and revealed damage related to seizure severity. Both AC-kainic and Kainic rats having non-convulsive seizures had little or no damage to CA1, CA2, CA3 or the dentate gyrus. There was some minor thinning of CA1 in a small number of animals in both groups but the majority of the animals had no apparent damage (Figure 4.7A-B). A volumetric analysis was performed to quantify lesion damage and found no difference in hippocampal damage between AC-kainic or Kainic rats without convulsions (AC-kainic: $4.48+/-4.16 \mathrm{~mm}^{2}$ and kainic: $0.00+/-0.00 \mathrm{~mm}^{2}$ ). A one way ANOVA was 
performed and found no effect of group $[F(1,15)=2.83, p=0.11]$. Both AC-kainic and Kainic rats that had convulsions showed the characteristic damage to CA1 and CA3 that results from systemic kainic acid injection (data not shown). AC-kainic rats appeared to have greater amounts of damage than sham operated Kainic rats; however, the small number of surviving rats with convulsions did not allow for a quantitative comparison of hippocampal damage between groups.

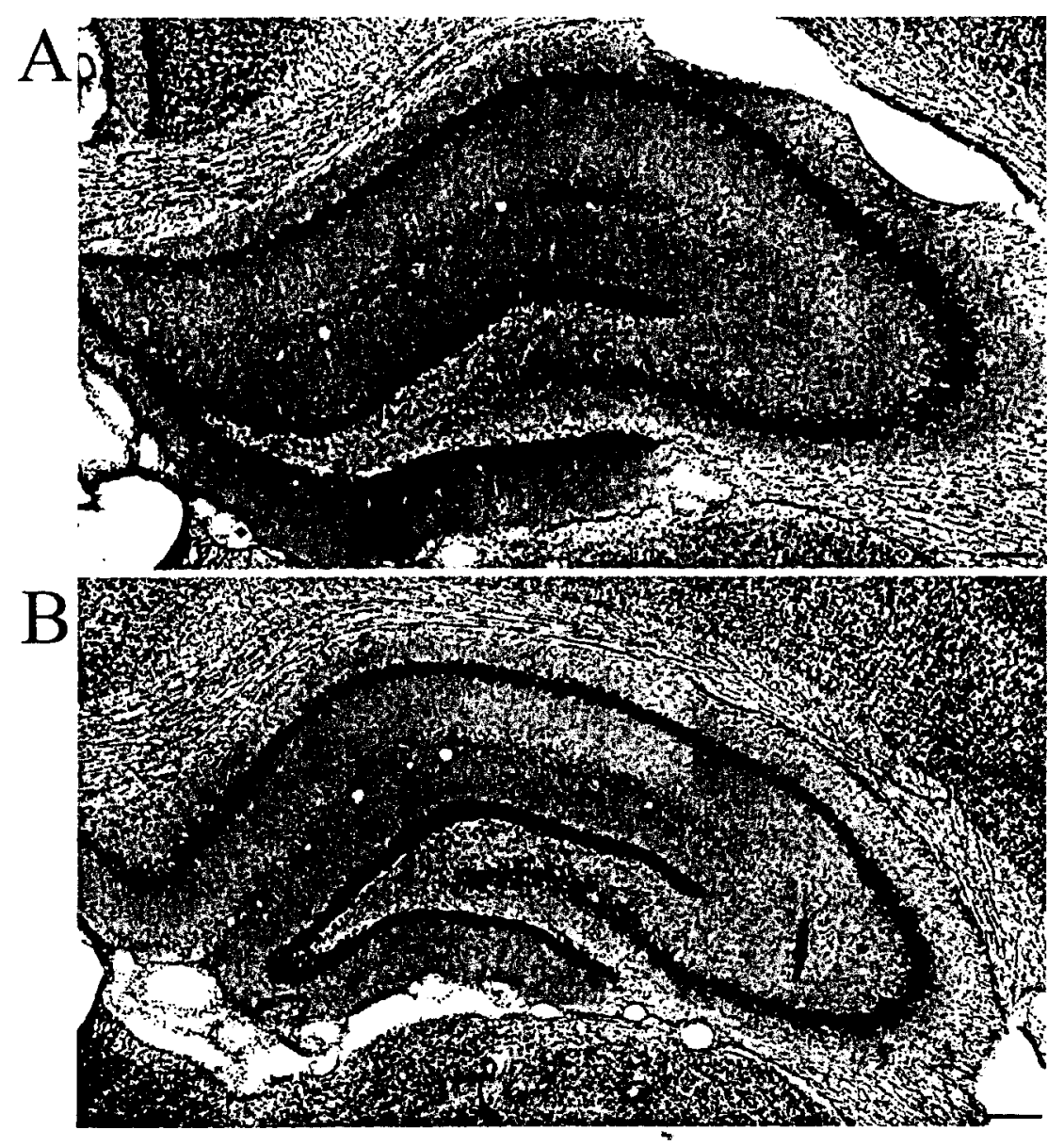

Figure 4.7: Photomicrographs (5x) of Cresyl Violet stained hippocampal sections from a Kainic rat without convulsions (A) and a AC-kainic rat without convulsions (B). Both groups show no damage or disruption to the hippocampal cell layers. Scale bars, $0.1 \mathrm{~mm}$. 


\section{Discussion}

The results of the present study show an interesting interaction between the cholinergic system and seizure activity on learning and memory. Previous work in our laboratory has shown no functional impairment on this task following cholinergic lesions alone (Craig and McDonald, 2006; Craig et al., 2008) which is consistent with the literature (Baxter and Gallagher, 1996; Frielingsdorf et al., 2005). The present study reports no deficit following the low dose of kainic acid used in sham operated animals, which confirms previous behavioural studies of rats injected with non-convulsive doses of kainic acid (Gobbo and O'Mara, 2004). Interestingly, in the present study, the combination of these two factors results in a significant impairment on the spatial water maze. The lesioned animals with convulsions performed at a level consistent with reports on rats following convulsive doses of kainic acid (data not shown). Gayoso et al. (1994) showed that while animals treated with a convulsive dose of kainic acid were initially severely impaired they were able to learn the task after intensive training. This is supported in my study, where the shortened original location training stage revealed the greatest difference between groups, but during new location training (massed training in a single session), both subgroups of the AC-kainic group performed at a similar level likely due to the extensive training period (data not shown). Nonetheless, all AC-kainic rats were impaired compared to Control, AC and Kainic rats. The novel finding here is the deficit observed in my non-convulsive lesioned rats. The combination of these two factors (cholinergic depletion and seizures) significantly impairs performance, while either factor alone has no effect. 
It is important to note that the behavioural deficit observed here was not due to seizure severity. Gobbo and O'Mara (2004) showed that following a low dose of kainic acid, which causes non-convulsive seizures, there were no impairments when rats were trained and tested in the water maze, whilst in animals exhibiting convulsive seizures there were distinct impairments. Although, the animals having convulsive seizures in my study performed worse than those having non-convulsive seizures, there was still a significant difference between the AC-kainic and Kainic groups on all aspects of the water maze task. This lends support to McDonald's co-factors theory (McDonald, 2002) that defines risk factors for $\mathrm{AD}$ as either passive factors that can increase the vulnerability of hippocampal neurons, yet have no noticeable effect on hippocampal memory, or active factors that have the potential to directly damage neurons and impair memory.

Here, the cholinergic depletion is acting as a passive factor by increasing the vulnerability of hippocampal neurons to an active factor (kainic acid induced seizures). I used a non-convulsive dose of kainic acid that does not affect spatial memory, to allow for the appearance of impairment in the cholinergic depleted rats, without the risk of masking by a previously existing deficit. My finding suggests that depleted rats were more vulnerable than sham operated rats to the effects of kainic acid on learning and memory. There was little gross neuronal damage observed in the hippocampus of depleted rats that could explain the observed impairment; however, the presence of this spatial memory deficit suggests a more subtle mechanism is being affected by these two co-factors leading to the disruption of hippocampal processing in rats following cholinergic depletion and kainic acid. While there are many studies that have correlated the amount of brain damage with the extent of behavioural impairment (Kiyota et al., 
1991; Nedelmann et al., 2007), there have also been a significant number of studies reporting a behavioural deficit in the absence of increased damage (Jaspers et al., 1990; Walh et al., 1991; Yamaguchi et al., 1995). The observation that performance is not always correlated with the amount of damage stresses the importance of using both histological and behavioural measures when assessing impairment and the value of future studies designed to detect subtle changes in hippocampal processing.

\section{Cholinergic depletion and seizure severity}

Several researchers have found an enhancement of hippocampal seizure activity or increased vulnerability to seizures following cholinergic depletion (Kokaia et al., 1996; Ferencz et al., 1997; Silveira et al., 2000; Ferencz et al., 2001).The mechanism by which cholinergic depletion is thought to influence seizure activity is via the cholinergic projections from the medial septum to the hippocampus. These projections play an important role in hippocampal excitability. Jouvenceau et al. $(1996,1997)$ demonstrated that removal of the cholinergic projections to the hippocampus resulted in an enhanced response to glutamate in a slice preparation, even though GABA-ergic and inhibitory responses remained normal. This agrees with the increased susceptibility to kindled, pentylenetetrazole- or flurothyl- induced seizures in animals lesioned with IgG SAP where the GABA-ergic projections remain intact (Kokaia et al., 1996; Silveira et al., 2000). Furthermore, the finding that the progression of kindling is unaltered in rats following cholinergic depletion suggests a quantitative change in excitability, rather than a qualitative change in connectivity (Kokaia et al., 1996; Ferencz et al., 2001). Taken together these findings would have predicted an increased susceptibility to kainic acid (a glutamate agonist) in the lesioned animals used in the current study, but this was not the 
case. Although there was a trend towards increased seizure severity in rats following cholinergic depletion in my study, this was not significant.

There are a few possible explanations for not observing increased seizure severity in my lesioned rats. First, I specifically damaged cholinergic neurons of the medial septum and this may not have been sufficient to lower the seizure threshold to systemic kainic acid. While a specific lesion of medial septal cholinergic neurons accelerates hippocampal kindling (Ferencz et al., 2001), reports examining generalized seizures induced by treatment with flurothyl or pentylenetetrazole have only been carried out in rats with complete lesions of the basal forebrain cholinergic neurons that project to the cortex as well as the hippocampus (Silveira et al., 2000). It is possible that the additional cortical depletion may create a more permissive environment for the spread of seizure activity leading to an increased response to seizures induced by systemic administration of epileptogenic chemicals. Alternatively, this is the first study to examine the effects of a non-convulsive dose of kainic acid in cholinergic depleted rats. The low dose used may not have been close enough to the convulsive threshold to allow me to see any effect of cholinergic depletion.

I have chosen to look at the interaction between cholinergic depletion and seizures because of the clinical observation of increased occurrences of seizures in patients suffering from $\mathrm{AD}$, and increased risk of $\mathrm{AD}$ in individuals with epilepsy. The increased incidence of seizures in $\mathrm{AD}$ patients is likely due to a number of co-existing risk factors, not only cholinergic depletion of the medial septum as was modeled here. However, I was interested to see whether there was an interaction between these two factors in the development of cognitive impairment. While I did not observe increased seizure 
susceptibility in my model, I did see a deficit in spatial memory suggesting that hippocampal impairment was a result of the interaction between these risk factors. It is possible that additional doses of kainic acid would accelerate epileptogenesis in cholinergic depleted rats and lead to increased neuronal damage, but future studies are required to fully understand the relationship between cholinergic neurons of the medial septum and the response to kainic acid.

\section{The excitotoxicity theory of neurodegenerative diseases}

The excitoxicity theory of neurodengerative diseases has received a lot of attention. Essentially, this theory suggests that increases in glutamate leads to cell death, which releases more glutamate in a continual cycle. The detrimental effects of cholinergic depletion and seizures fit in nicely with this theory. Cholinergic depletion enhances the release of hippocampal glutamate, while simultaneously increasing the response to glutamate. This combined with seizure activity, will further increase the amount of glutamate, leading to more extensive hippocampal damage than either factor alone. Lothman and Collins (1981) have reported that it is the convulsive nature of the seizures that is required for neuronal cell death, rather than the absolute dose of kainic acid. The low dose of kainic acid used in the current study did not produce hippocampal damage in the absence of a convulsive seizure, but in combination with cholinergic depletion of the medial septum, does lead to behavioural deficits on a task reliant on the hippocampus. In addition, I have observed a trend towards increased hippocampal damage in lesioned rats that had a convulsive seizure compared to non-lesioned rats (data not shown). Over time seizures may become more frequent and would increase the amount of damage to other areas of the brain, negatively affecting different memory 
systems located in anatomically distinct areas. Geddes and Cotman, (1986) reported that $\mathrm{AD}$ patients had an expanded distribution of the kainate receptor field in the dentate gyrus, making them more sensitive to the excitotoxic effects of glutamate. The recent effectiveness of the non competitive glutamate antagonist, memantine, in treating moderate to severe cases of $\mathrm{AD}$ lends further support to this theory (Reisberg et al., 2003; Tariot et al., 2004).

\section{Support for the multiple combinations of co-factor theory of aging}

This study provides support for the co-factor theory proposed by McDonald (2002). By combining two distinct factors at a dosage that have no effect on performance on their own, I observe a deficit on hippocampal specific behaviour in the absence of any gross neuronal damage. Additional support for this model has been provided combining a number of other risk factors for $\mathrm{AD}$ such as aging and mild ischemia (Driscoll et al., 2007), stress and mild ischemia (McDonald et al., 2008) as well as cholinergic depletion and stress (Craig et al., 2008). While the effect of these combinations on hippocampal pathology can vary between dramatically ramping up lesion size (Driscoll et al., 2007; McDonald et al., 2008), to no obvious damage (Craig et al., 2008), the appearance of a deficit on hippocampal based tasks remains constant. Taken together, these studies suggest that although the many risk factors for $\mathrm{AD}$ are all capable of producing cognitive impairment, the underlying mechanism or extent of neuronal damage varies depending on the risk factors present. This has important implication for designing both preventative and reactive treatment plans for individuals at risk for, or suffering from $\mathrm{AD}$. 


\section{Conclusion}

Here I show that the combination of two risk factors for $\mathrm{AD}$ (cholinergic depletion and non-convulsive seizures) can lead to cognitive deficits on a task designed to test hippocampal function. I believe that the loss of cholinergic projections to the hippocampus have rendered this region increasingly vulnerable to the effects of seizure activity on learning and memory. While I am not arguing that seizures 'cause' $\mathrm{AD}$, or dementia, I am proposing that the presence of late onset seizures are contributing to the memory deficit and eventual hippocampal damage of an individual who may be in the early stages of $\mathrm{AD}$, or simply, due to a depletion of acetylcholine, be more vulnerable to the effects of excitotoxic activity in the brain. For example an individual may present as non- or mildly demented for years with a depleted cholinergic system; then, following the occurrence of an unprovoked spontaneous seizure, would likely have an exaggerated response in terms of cognitive impairment, seizure severity and/or hippocampal damage. This could lead to the expression of early signs of a subtype of $\mathrm{AD}$. This individual would require a different treatment program from an individual with a preponderance of genetic factors even though both patients ultimately are diagnosed with $\mathrm{AD}$. 


\section{Chapter 5}

Selective lesion of medial septal cholinergic neurons followed by a mini-stroke impairs spatial learning in rats

Modified from a paper submitted to Experimental Brian Research

by Laura A. Craig, Nancy S. Hong, Joelle Kopp and Robert J. McDonald in 2008 


\begin{abstract}
Reduced levels of hippocampal acetylcholine are a common finding in patients diagnosed with Alzheimer's disease, but it remains unclear what role this depletion plays in the development of dementia. It is possible that reduced levels of acetylcholine increases the vulnerability of hippocampal neurons to future insults, which could lead to neuronal death and cognitive impairment. One insult that is commonly observed in the demented elderly and often co-exists with Alzheimer's disease is stroke. In the current experiment, I used the immunotoxin 192 IgG Saporin to specifically lesion the cholinergic neurons of the medial septum that project to the hippocampus. I then explored the effects of small, localized strokes in the hippocampus on a spatial learning task. The combination of cholinergic depletion and stroke resulted in significant impairment on the spatial water maze compared to the performance of rats receiving either factor alone. Volumetric analysis of the hippocampus revealed no difference in the overall lesion size of strokeonly or combined (cholinergic depletion and stroke) rats, suggesting that a more subtle mechanism is responsible for the observed impairment. I propose that healthy hippocampal neurons may normally be able to withstand, and compensate for a small ischemic insult. However, in the absence of cholinergic projections from the medial septum, these compensatory processes in the hippocampus may be compromised resulting in the spatial learning impairment reported here. This suggests an association between the cholinergic depletion observed during aging and the potential for functional recovery following stroke.
\end{abstract}




\section{Introduction}

The characteristic pathology of Alzheimer's disease (AD) (amyloid plaques, neuritic tangles and reduction in acetylcholine levels) has been well described over the past 30 years; yet the etiology of this disorder remains a mystery. Several single factor theories of causation have been proposed but the most plausible theories describe a combination of multiple factors that can produce specific sub-types of this disorder. The combination of factors present will consequently affect the diagnosis and future treatment choices for each group of patients (McDonald, 2002).

One of the risk factors for $\mathrm{AD}$ is a loss of cholinergic neurons of the basal forebrain and there is a significant decline in acetylcholine (ACh) in the brains of patients with AD (Davies and Maloney, 1976; Gallagher and Colombo, 1995; Mesulam, 2004). Unfortunately, treating $\mathrm{AD}$ patients with cholinergic agonists has had varying success at slowing the progression of this disorder, suggesting that the loss of ACh is not causative on its own. Nevertheless, the consistency of this depletion in $\mathrm{AD}$ patients suggests that levels of ACh may have an important role to play in the development of this disorder, especially in the early stages. In addition to human $\mathrm{AD}$ patients, many transgenic mice developed to model $\mathrm{AD}$, exhibit significantly reduced levels of $\mathrm{ACh}$ in the brain, particularly in the hippocampus further strengthening the link between $\mathrm{AD}$ and cholinergic depletion (Morley, 2002; Belluci et al., 20̄06).

Another risk factor that has been consistently linked with dementia is stroke. Cortical and subcortical infarcts resulting from "silent" stroke have become increasingly common in the elderly population (Shi et al., 2000). While current diagnoses of $\mathrm{AD}$ specifically exclude cases with co-existing vascular disease, it is impossible to ignore the 
significance of the finding that mixed dementia (both vascular and $A D$ pathology in an individual) is the most common form of dementia (Scheinder et al., 2007), and that the presence of vascular disease, or stroke increases the chances of developing $\mathrm{AD}$ and the eventual severity of the disorder (Snowden et al., 1997). Global ischemia and lacunar stroke have been found to preferentially target the hippocampus resulting in increased levels of apoptosis and hippocampal atrophy (Kirino and Sano, 1984; Honkaniemi et al., 1996; Back et al., 2004; Grau-Olivares et al., 2007). Song et al. (2007) found that a large percentage of $\mathrm{AD}$ patients with infarcts exhibited more rapid decline of cognitive abilities than those without infarcts. Taken together these studies suggest that hippocampal damage resulting from stroke could accelerate the appearance of dementia in preclinical $\mathrm{AD}$ patients.

Here I examined the behavioural effects of two common risk factors, cholinergic depletion and stroke that both target the hippocampus, on spatial and non-spatial versions of the water maze task. I predicted that either the stroke or cholinergic depletion alone would have little or no effect on behaviour, while the combination of the two factors (cholinergic depletion and stroke) would result in increased hippocampal damage in response to stroke and a corresponding impairment on the spatial version of the water maze task.

\section{Materials and Methods}

\section{Animals}

Twenty four male Long Evans hooded rats (300-400g) were obtained from Charles River (Saint-Constant, QC) and used for this experiment. Rats were divided into four groups: AC $(n=9)$ received a cholinergic depletion followed two weeks later by a 
sham stroke; Stroke $(n=8)$ received a sham depletion followed by stroke; AC-stroke $(n=8)$ received a cholinergic depletion followed by stroke; and Control $(n=7)$ received a sham depletion followed by a sham stroke. Animals were pair housed with food and water ad libitum, on a 12 hour light/dark cycle with lights off at 19:30. All animals were handled in accordance to guidelines set out by the Canadian Council for Animal Care.

\section{Surgery}

Surgery was performed under Isoflurane anaesthesia $(4 \%$ with $2.0 \mathrm{~L} / \mathrm{min}$ of oxygen for induction and $2 \%$ after surgical plane was established) in a standard stereotaxic apparatus. An incision was made in the scalp and periosteum down the midline. The fascia was pushed to the edges of the skull with a sterile gauze swab and the skin retracted to expose the skull surface. Holes were drilled into the skull using a 1 mm drill bit attached to a high speed drill. Following surgery all animals were given a single dose of buprenorphine (Temgesic; $0.1 \mathrm{mg} / \mathrm{kg}$ subcutaneously) as an analgesic.

\section{Cholinergic depletion}

The specific neurotoxin 192 IgG Saporin (IgG SAP; Chemicon) has been commonly used to induce selective cholinergic lesions in rats depending on the site of infusion. I chose to damage cholinergic neurons in the medial septum and vertical limb of the diagonal band of Broca (MS/VDB) which leads to a reduction in levels of hippocampal acetylcholinesterase (AChE). I lowered a 30 gauge cannula, through holes drilled in the skull, into the MS/VDB (AP +0.45; ML +/-0.6; DV -6.6 and $-8.0 \mathrm{~mm}$ from bregma and the skull) and bilaterally infused IgG SAP $[0.15 \mu \mathrm{g} / \mu \mathrm{l}$ dissolved in sterile $1 \mathrm{x}$ phosphate buffer saline (PBS; $\mathrm{pH} 7.4$ )] at a rate of $0.05 \mu \mathrm{l} / \mathrm{min}$ using a Harvard minipump. Each animal received a total volume of $0.3 \mu 1$ per side at the first injection site 
(DV -8.0), and $0.2 \mu \mathrm{l}$ per side at the second site (DV -6.6). The cannula was left in place for six and four minutes after the first and second injection respectively to ensure diffusion of the toxin. Sham operated animals underwent an identical procedure, but were infused with sterile $1 \times$ PBS instead of IgG SAP.

Stroke

The vasoconstrictor Endothelin-1(ET-1; human, porcine; Sigma) was used to create a small, localized stroke in the hippocampus. A prior, unpublished, experiment was carried out to test different groups of rats on the hidden platform version of the Morris water maze following different concentrations of ET-1 stroke. The lowest concentration (7.5 pmol used here) was chosen because it did not result in functional impairments on the standard, spatial version of the water task. I lowered a 30 gauge cannula, through holes drilled in the skull in to the dorsal (AP -4.1; $\mathrm{ML}+1-3.0 ; \mathrm{DV}-3.7 \mathrm{~mm}$ from bregma and the skull respectively) and ventral hippocampus (AP -5.2; $\mathrm{ML}+/-5.3$; DV -7.0 mm from bregma and the skull respectively) and bilaterally infused ET-1 (7.5 pmol dissolved in sterile saline) at a rate of $0.1 \mu \mathrm{l} / \mathrm{min}$ using a Harvard mini-pump. Each animal received a total volume of $5 \mu \mathrm{l}$ to each site. The cannula was then left in place for 10 minutes after each infusion to ensure diffusion of the toxin. Sham operated animals underwent an identical procedure, but were infused with sterile saline instead of ET-1. Following surgery animals were given buprenorphine (Temgesic; $0.1 \mathrm{mg} / \mathrm{kg}$ subcutaneously) as an analgesic.

\section{Behavioural Testing}

Behavioural testing began one week following the second surgery (stroke or sham stroke) and consisted of six days on the Rapid Acquisition Task to test for deficits in 
hippocampal processing, followed by two days on a visual platform task to control for sensory, motor or motivational deficits.

\section{Water maze: Rapid Acquisition Task}

I used a three stage spatial version of the water maze task (McDonald et al., 2005). A white plastic circular pool $1.4 \mathrm{~m}$ in diameter and $40 \mathrm{~cm}$ deep was filled to within $20 \mathrm{~cm}$ of the top of the wall with water $\left(20-22^{\circ} \mathrm{C}\right)$ made opaque by adding skim milk powder. The invisible platform was $12 \mathrm{~cm}$ in diameter and made of white Plexiglas with holes drilled into the top of it to provide grip for the animals. During training the platform was submerged $2 \mathrm{~cm}$ under water. Extra-maze cues in the training room included five posters of different sizes and orientations mounted on three of the four walls, a computer rack, a door, an animal rack and the experimenter. Four different start points were used [N, S, E, W (not true compass headings)], equally spaced around the pool. The order was randomly selected for each day and each start point was used twice for days one to four and days six to eight. On day five only the three start points farthest from the platform were used (N, E, S) with the initial start point repeated at the end for a total of 16 trials.

In the first stage (Original location training) rats were trained over four days for eight trials a day, to swim to a fixed, spatial location. Twenty four hours after completion of stage one the second stage (New location training) began. This stage consisted of new spatial location training as animals were re-trained to swim to the new platform location over sixteen trials within two hours on a single day. The third stage consisted of two parts: a probe test and re-training to original location, both of which occurred 24 hours after mass training and each animal was given a 60 second trial with the platform 
removed from the pool. The platform was then returned to the pool in its original (stage one) location and each animal was given seven additional trials in which to re-learn the original location.

This version of the water maze was selected due to its sensitivity to hippocampal damage. The first stage allowed me to identify impairment in acquisition and short term memory of an invariant location. If no differences were apparent, the second stage would test the ability of the hippocampus to rapidly form a novel representation in a familiar context. The third stage allowed me to test spatial memory for the previously learnt locations and determine which of these locations the subject had formed the strongest representation. The second part of stage three allowed me to ask how the massed new location training during stage two affected re-training to the original location (McDonald et al., 2005).

\section{Water maze: Visible platform}

The visible platform version of the water maze task was used as a test of general performance that could control for potential sensory, motor and/or motivation deficits. The same pool and room used for the spatial water maze task was used for this task, except that the platform was made visible by attaching a $6 \mathrm{~cm}$ high black block to the top of the hidden platform. This block was raised $2 \mathrm{~cm}$ out of the water, allowing rats to easily see the platform. The visible platform task was carried out on days seven and eight and consisted of one training session each day. Each session consisted of four trials (lasting 30 seconds) using each start point (N, W, E, S) once. The platform was placed in a novel location at the start of each training session to discourage the use of a spatial strategy in the second session. 
Data collection: A computer based rat tracker (Ethovision) was used to collect and analyze water maze data obtained from an overhead video camera. For the training trials, the measures of performance were swim time, defined as the latency between release and escape onto the platform and length of the path taken to find the platform. Path length is a sensitive measure that can control for potential differences in swim speed. If a rat did not reach the platform 60 seconds after release, a latency of 60 seconds was assigned, and the rat was guided manually to the platform and allowed to remain on the platform for 10 seconds before being removed to a holding cage until the next trial. For the probe test, platform preference was used as a measure of performance. The percentage of time the rat spent in each quadrant, as well as the number of times the rat crossed each of the platform locations was recorded. During the visible platform task, latency to platform, path length and swim speed were assessed and compared from day one to day two.

\section{Histology}

Following behavioural testing all animals were sacrificed with an overdose of Euthanol and transcardially perfused with 1x PBS (pH 7.4) followed by $4 \%(w / v)$ paraformaldehyde (PFA). The brains were carefully extracted and placed in $4 \%(\mathrm{w} / \mathrm{v})$ PFA at $4^{\circ} \mathrm{C}$ for 24 hours. Brains were then transferred to a $30 \%(w / v)$ sucrose solution, containing $0.02 \%(\mathrm{w} / \mathrm{v})$ sodium azide at $4^{\circ} \mathrm{C}$ for at least three days. Brains were sectioned on a freezing cryostat at $40 \mu \mathrm{m}$ in a series of five. The first two sections of each series were mounted onto $1 \%(\mathrm{w} / \mathrm{v})$ gelatin $/ 0.2 \%(\mathrm{w} / \mathrm{v})$ chromalum coated slides, while the third, fourth, and fifth series were stored in $1 \mathrm{x}$ PBS containing $0.02 \%(\mathrm{w} / \mathrm{v})$ sodium azide at $4^{\circ} \mathrm{C}$ until being processed for immunohistochemistry. The fifth series was not used in this experiment and was stored for future analyses. 


\section{Cresyl violet staining and volumetrics}

The first series was stained with Cresyl violet and volumetric analysis was used to determine the extent of damage to the hippocampus. Working in a rostral-caudal direction, coronal sections were examined and at the first sign of hippocampal damage pictures were taken under a $10 \mathrm{x}$ objective to document the extent of the lesion. I continued to take pictures of every fifth section leaving approximately $200 \mu \mathrm{m}$ between sections sampled to ensure no cell, or hippocampal region was counted twice. After all documentation of the damage for each subject in all of the sections was obtained (usually three to four sections), volumetric analysis was completed manually using an AxioVision microscope with associated Axio Imager analysis software (version 4.3). The extent of damage in the CA fields and dentate gyrus of the hippocampus were traced and a calculation of the volume of the lesion within the traced areas was obtained. The values from the traced areas were calculated by the computer software in millimetres squared. The total volume of damage for each animal was calculated and used to obtain an average for each group. This method of analysis has been used previously and produces an accurate estimate of hippocampal damage following focal stroke in the rat (Driscoll et al., 2007; McDonald et al., 2008).

\section{AChE staining}

The second series was stained for $\mathrm{AChE}$ to get a qualitative image of cholinergic depletion in the hippocampus. I treated the slides for 30 minutes with $6.24 \mathrm{mg}$ tetraisopropylpyrophosphoramide (Sigma) dissolved in $200 \mathrm{~mL}$ of distilled water, then rinsed the slides twice with distilled water and incubated them on a shaker table for four hours in a reaction mixture containing $5.72 \mathrm{~g}$ Trizma maleate, $2.04 \mathrm{~g}$ Trizma base, 100 
mg acetylthiocholine iodide, $294 \mathrm{mg}$ dihydrous trisodium citrate, $150 \mathrm{mg}$ anhydrous cupric sulphate, $32.8 \mathrm{mg}$ potassium ferricyanide dissolved in $200 \mathrm{~mL}$ distilled water (all chemicals were obtained from Sigma). Slides were rinsed twice with distilled water, and left to dry overnight, then cleared with HemoDe (Sigma) and coverslipped with Permount (Sigma) the next day.

\section{Immunohistochemistry}

The third and fourth series were processed for immunohistochemistry to determine the completeness and specificity of the cholinergic lesion in the medial septum. An antibody raised against parvalbumin was used to label $\gamma$-aminobutyric acid (GABA)-ergic neurons, and one against choline acetyltransferase (ChAT) was used to label cholinergic neurons. Free floating sections were rinsed in $1 \times$ PBS, and then placed in a $3 \%(\mathrm{v} / \mathrm{v}) \mathrm{H}_{2} \mathrm{O}_{2}$ solution for four minutes to block endogenous peroxidases. After three washes in 1x PBS, sections were blocked for 30 minutes in $15 \%(v / v)$ normal horse serum (for anti-parvalbumin; Vector Labs) or 15\% (v/v) normal rabbit serum (for antiChAT; Vector Labs). Sections were incubated overnight in primary antibody [anti-ChAT polyclonal, 1:1000 (Chemicon) or anti-parvalbumin monoclonal, 1:5000 (Sigma)] diluted in $1 \mathrm{x}$ PBS with $7.5 \%(\mathrm{v} / \mathrm{v})$ serum (horse for anti-parvalbumin; rabbit for anti-ChAT). Sections were then washed three times in 1x PBS and incubated for 30 minutes in a biotinylated secondary antibody $[\mathrm{ABC}$ Elite Kit (mouse for parvalbumin and goat for ChAT), Vector Labs], washed three times, incubated for 30 minutes with AB reagent (ABC Elite Kit, Vector Labs), washed three times then developed with diaminobenzidine (DAB) substrate (Vector Labs) according to the manufacturer's instructions. Sections were then mounted onto $1 \%(\mathrm{w} / \mathrm{v})$ gelatin $/ 0.2 \%(\mathrm{w} / \mathrm{v})$ chromalum coated slides, air dried 
overnight, dehydrated in alcohol, cleared in HemoDe (Sigma) and coverslipped with Permount (Sigma).

Cell counts: Under light microscopy (AxioVision) ChAT positive neurons were counted in both hemispheres of the MS/VDB. I chose two sections to quantify; the first, at the site of injection and the second, in a section $200 \mu \mathrm{m}$ caudal to the injection site. This quantification was not used as an accurate count of MS/VDB neurons, but instead to estimate the completeness of the lesion. Each hemisphere was counted separately to confirm lesion symmetry, after which counts for both hemispheres were then totaled for a representative cell count over the two sections. I combined the ChAT positive cell counts from the stroke and control groups, and used this average to determine the percentage of cell loss in the lesioned groups. Any animal with less than $35 \%$ cell loss was excluded from the experiment.

\section{Results}

Five rats assigned to the $\mathrm{AC}$ and $\mathrm{AC}$-stroke groups were removed from this experiment due to incomplete cholinergic lesions ( $<35 \%$ cell loss). The following histological and behavioural analyses include five animals in the AC group and seven rats in the AC-stroke group.

\section{Water maze: Rapid Acquisition Task}

\section{Original location training}

The AC-stroke group was significantly impaired on all days in their ability to learn the platform location during original location training when compared to the $\mathrm{AC}$, Stroke and Control groups (Figure 5.1A). However, the Stroke rats had slower latencies 
to find the platform on the final day of training suggesting impairment in these rats on this stage of training. The latency (in seconds) to find the platform was recorded and averaged over the eight daily trials to obtain a single daily latency score for each animal. The daily scores for all four days of original location training were subjected to a twoway mixed analysis of variance (ANOVA) with Group (AC-stroke versus AC versus Stroke versus Control) as the between subjects variable, and Training Day (four levels) as a repeated measure, and revealed significant effects of group $[F(3,23)=14.29, p<0.01]$, of day $[F(3,69)=75.17, p<0.01]$ and an interaction $[F(9,69)=2.19, p<0.05]$. By the final day of training AC-stroke rats were taking four times longer to find the platform $(23.98+/$ $3.99 \mathrm{sec})$ and Stroke rats were taking almost three times longer $(16.55+/-0.99 \mathrm{sec})$ than the averaged latency for $\mathrm{AC}$ and Control rats $(5.87+/-0.39 \mathrm{sec})$. Planned comparisons revealed that AC-stroke rats had significantly longer latencies on days two to four when compared to all other groups $\left(p^{\prime} s<0.05\right)$. I also found that Stroke rats had significantly longer latencies than either $\mathrm{AC}$ or Control rats on day four $(p<0.05)$, but there was still a significant difference between the latencies of Stroke rats and AC-stroke rats on this day $(p<0.05)$. 

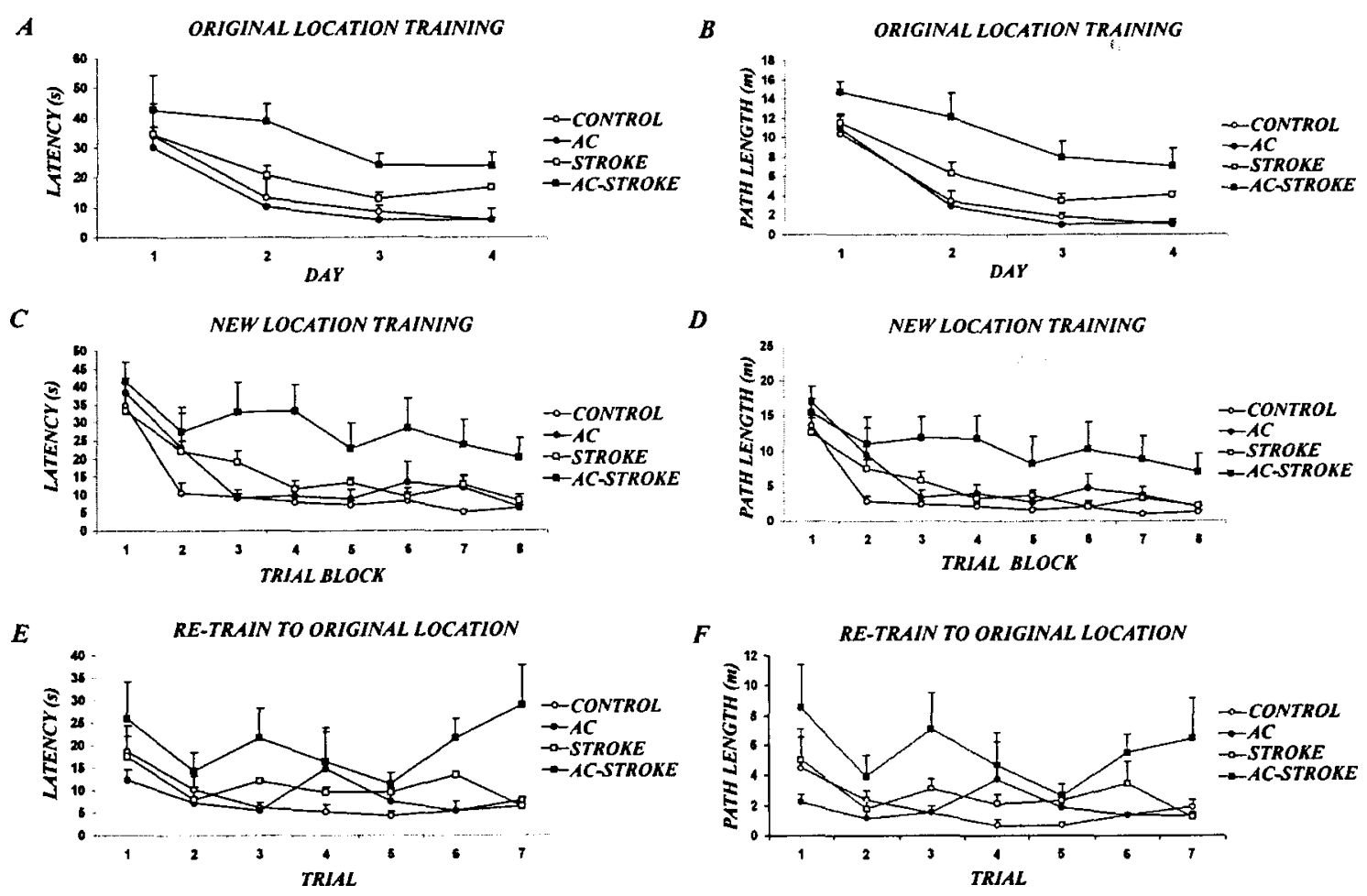

Figure 5.1: AC-stroke rats were impaired on all stages of the spatial water maze task as measured using latency to platform and path length as dependent variables. AC-stroke rats take longer to find the platform during original location training $(\mathrm{A}, \mathrm{B})$, new location training $(C, D)$ and re-training to the original location $(E, F)$. Data are given as means $+/-$ SEM.

To control for potential differences in swim speed that could account for this difference, I also analyzed path length and found a similar pattern of results. AC-stroke rats had significantly longer path lengths than all other groups; however, no impairment was observed in Stroke rats using this measure (5.1B). A two-way mixed ANOVA with Group as the between subjects variable and Training Day (four levels) as the repeated measure revealed a significant effect of group $[F(3,23)=10.23, p<0.001]$ and of day $[F(3,69)=69.49, p<0.001]$, but no interaction $[F(9,69)=1.16, p=0.34]$. Holm-Bonferroni post hocs showed that the path lengths of AC-stroke rats were significantly longer than the path lengths of all other groups ( $p$ 's $<0.05)$. 
New location training

The AC-stroke group was impaired in their ability to rapidly acquire a new platform location on this stage of the task when compared to all other groups (Figure 5.1C). By the final trial block, AC-stroke rats were still taking longer to find the platform $(20.08+/-5.00$ seconds $)$ than the averaged latency of AC and Control rats $(6.35+/-0.53$ seconds). Stroke rats performed at control levels during new location training $(8.35 \mathrm{H} /-$ 1.48 seconds). A two-way mixed ANOVA with Group as a between subjects variable and Trial Block (eight levels) as a repeated measure, was used to assess new location training and showed a significant effect of group $[F(3,23)=5.81, p<0.01]$ and of trial block $[F(7,161)=23.96, p<0.01]$, but no interaction $[F(21,161)=1.28, p=0.19]$. Holm-Bonferroni post hocs showed that the latencies of AC-stroke rats were significantly longer compared to the latencies of all other groups on this stage of the task $\left(p^{\prime} s<0.05\right)$.

I also assessed path length on this day and found AC-stroke rats had longer path lengths compared to Control, AC or Stroke rats (5.1D). A two-way mixed ANOVA with Group as the between subjects variable and Trial Block (eight levels) as the two-way mixed found a significant effect of group $[F(3,23)=5.03, p<0.01]$ and of trial block $[F(7,161)=23.55, p<0.001]$, but no interaction $[F(21,161)=1.17, p=0.29]$. HolmBonferroni post hocs showed that the path lengths of AC-stroke rats were significantly longer than the path lengths of all other groups $(p$ 's $<0.05)$.

Probe test

I found no differences between the groups on measures of quadrant preference or platform crossings. A two-way mixed ANOVA with Group as the between subjects variable and Quadrant Preference (new, original or other) as the repeated measure found 
no effect of group $[F(3,23)=0.18, p=0.91]$, quadrant $[F(2,46)=2.61, p=0.08]$ or interaction $[F(6,46)=0.125, p=0.30]$. The number of platform crossings was subjected to an ANOVA with Group as the between subjects variable and Crossing (new or original) as the dependent variable. This analysis found no effect of group $[F(3,23)=0.73, p=0.54]$, crossing $[F(1,23)=0.17, p=0.69]$ or interaction $[F(3,23)=0.64, p=0.60]$ (data not shown).

\section{Re-training to original location}

The results of the re-training to original location stage are displayed in Figure 5.1E and show that the AC-stroke group was impaired in their ability to re-learn the original location on this stage of the task when compared to all other groups. A two-way mixed ANOVA, with Group as the between subjects variable, and Trial (seven levels) as a repeated measure revealed significant effects of group $[F(3,23)=6.57, p<0.01]$, and of trial $[F(6,138)=2.48, p<0.05]$, but no interaction $[F(18,138)=0.96, p=0.50]$. By the final trial AC-stroke rats were taking almost four times as long to find the platform $(28.79+1-$ 8.06 seconds) as the averaged latency for $\mathrm{AC}$ and Control groups (7.16 +/- 0.99 seconds). Stroke rats were performing at control levels $(6.47+/-1.70$ seconds). Holm-Bonferroni post hocs showed that the latency to platform of AC-stroke rats was significantly longer than the latencies of all other groups on this stage of the task $\left(p^{\prime} s<0.05\right)$.

Path lengths were assessed during re-training to the original location and found AC-stroke rats to be impaired compared to all other groups (5.1F). A two-way mixed ANOVA with Group as the between subjects variable and Trial (seven levels) as the repeated measure revealed a significant effect of group $[F(3,23)=4.92, p<0.01]$ and of trial $[F(6,138)=2.75, p<0.05]$, but no interaction $[F(18,138)=0.84, p=0.65]$. Holm- 
Bonferroni post hocs showed the path lengths of AC-stroke rats were significantly longer than the path lengths of Control, $\mathrm{AC}$ or Stroke rats $\left(p^{\prime} s<0.05\right)$.

\section{Visible platform}

The results of this task are depicted in Figure 5.2. AC-stroke rats took longer than the $\mathrm{AC}$ and Stroke groups to locate the platform on day one, but were no different from Control rats (Control: $9.02+/-2.15$ seconds; AC: $6.03+/-0.36$ seconds; Stroke: $7.48+/-$ 0.99 seconds; AC-stroke: $11.67+/-1.77$ seconds). All groups showed improved performance from day one to day two, but AC and Stroke rats still escaped from the pool faster than control or AC-stroke rats (5.2A). The latency (in seconds) to find the platform and escape from the pool were averaged over the four daily trials, and a two-way mixed ANOVA, with Group as the between subjects variable and Training Day (two levels) as the repeated measure, revealed significant effects of group $[F(3,23)=3.42, p<0.05]$, and of day $[F(1,23)=10.10, p<0.01]$, but no interaction $[F(3,23)=0.81, p=0.50]$. HolmBonferroni post hocs showed that the latency to platform of AC-stroke rats was significantly longer than $\mathrm{AC}$ and Stroke rats ( $p$ 's<0.05), but was not different from Control rats.

Analysis of path length showed a similar pattern of results as AC-stroke rats took a longer path to reach the visible platform, but once again showed a significant improvement by day two suggesting that they were able to learn the task and were motivated to escape from the pool (5.2B). A two-way mixed ANOVA with Group as the between subjects variable and Training Day (two levels) as the repeated measure revealed a significant effect of group $[F(3,23)=3.97, p<0.05]$ and of day $[F(1,23)=10.59, p<0.01]$ but no interaction $[F(3,23)=0.81, p=0.50]$. Holm-Bonferroni post hocs found that the path 
lengths of $\mathrm{AC}$-stroke rats were significantly longer than the path lengths of Control, $\mathrm{AC}$ and Stroke rats $\left(p^{\prime}{ }^{\prime}<0.05\right)$.
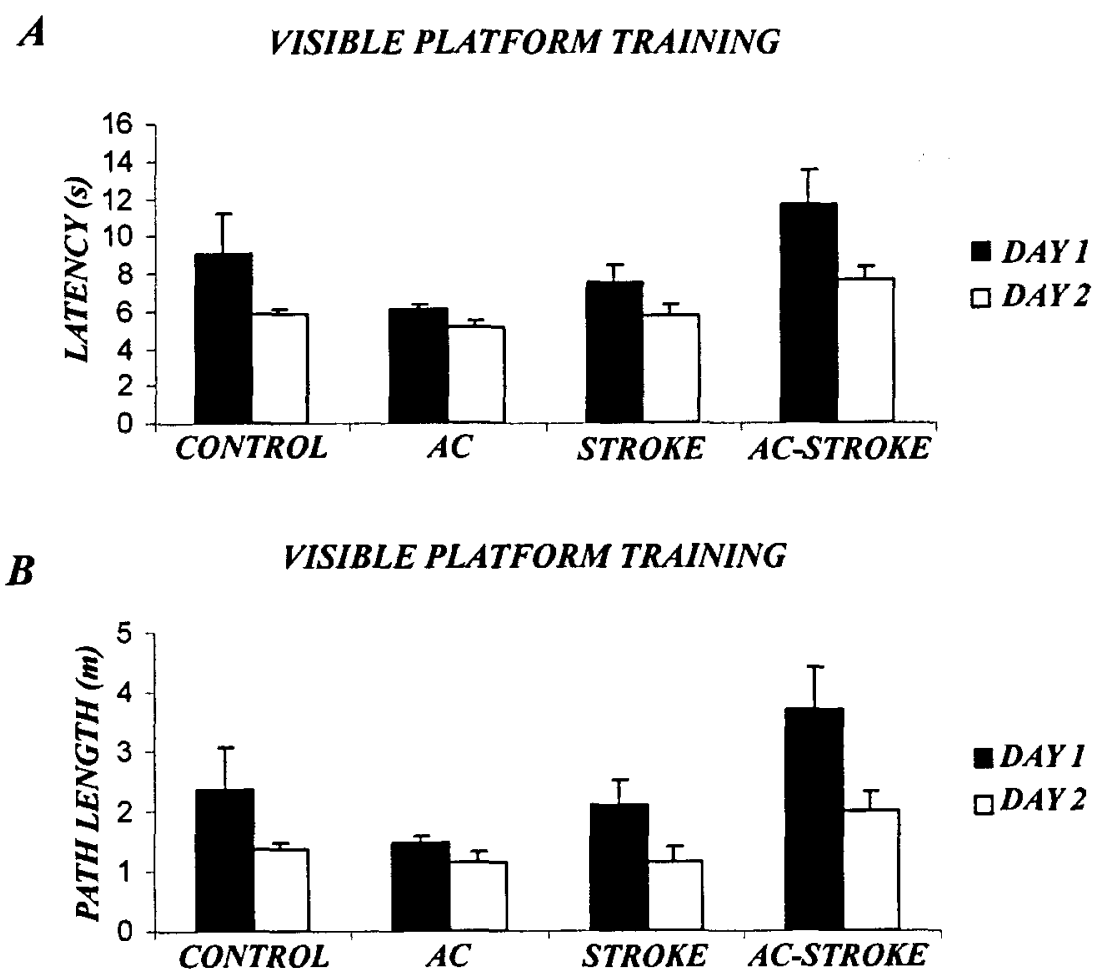

Figure 5.2: AC-stroke rats took longer to reach the platform during visible platform training, but they did show improvement by day two suggesting they were motivated to escape the pool $(\mathrm{A}, \mathrm{B})$. Data are given as means $+/$ - SEM.

An analysis of swim speed during visible platform training found $\mathrm{AC}$ rats were significantly faster than Control and Stroke rats, but all other groups had similar swim speeds (control: $0.20+/-0.01 \mathrm{~m} / \mathrm{s}$; AC: $0.26+/-0.28 \mathrm{~m} / \mathrm{s}$; stroke: $0.20+/-0.01 \mathrm{~m} / \mathrm{s}$; ACstroke: $0.23+/-0.12 \mathrm{~m} / \mathrm{s}$ ). A one way ANOVA revealed a significant effect of group $[F(3,23)=4.12, p<0.05]$. Holm-Bonferroni post hocs found that the swim speed of AC rats

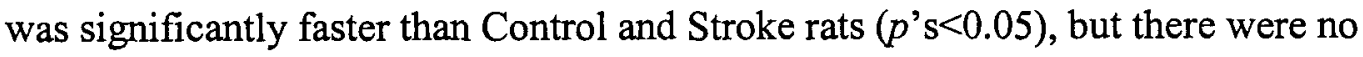
differences in swim speed between the other groups. 


\section{Histology}

Quantification of cholinergic lesion

I confirmed the completeness and specificity of the cholinergic lesion in the medial septum of both $\mathrm{AC}$ and $\mathrm{AC}$-stroke groups through $\mathrm{AChE}$ staining and immunohistochemistry for ChAT and parvalbumin. Lesioned animals had very few ChAT positive cholinergic neurons (Figure 5.3A-C) combined with intact parvalbumin labeled GABA-ergic neurons (5.3D-F) confirming the specificity of the cholinergic lesion. The $\mathrm{AChE}$ staining clearly showed a depletion of cholinergic activity in the hippocampus in all lesioned animals compared to the sham operated groups (5.3G-I).

I quantified the extent of the cholinergic lesion by comparing the ChAT positive cell counts between groups. To control for any possible effects of stroke on the number of cholinergic neurons in the medial septum, I used the ChAT positive cell counts from the Stroke group to determine a percentage of cell loss for the AC-stroke group, and used the ChAT positive cell counts from the Control group to determine a percentage of cell loss for $\mathrm{AC}$ group. I found $80.31+/-0.47 \%$ cell loss in the AC-stroke group and $67.49+/$ $29.14 \%$ cell loss in the $\mathrm{AC}$ group (One $\mathrm{AC}$-stroke rat and four $\mathrm{AC}$ rats had incomplete lesions with less than $35 \%$ depletion and were removed from the study). There were significantly fewer cholinergic neurons in the $\mathrm{AC}$ and $\mathrm{AC}$-stroke groups compared to the Stroke and Control groups (Table 5.1). A one way ANOVA revealed a significant effect of group $[F(3,23)=17.66, p<0.01]$. Holm-Bonferroni post hocs showed that there was no significant difference in the extent of the depletion for AC and AC-stroke groups, and that both groups had significantly fewer ChAT positive cells than either the Stroke or Control groups $(p ' s<0.05)$. 

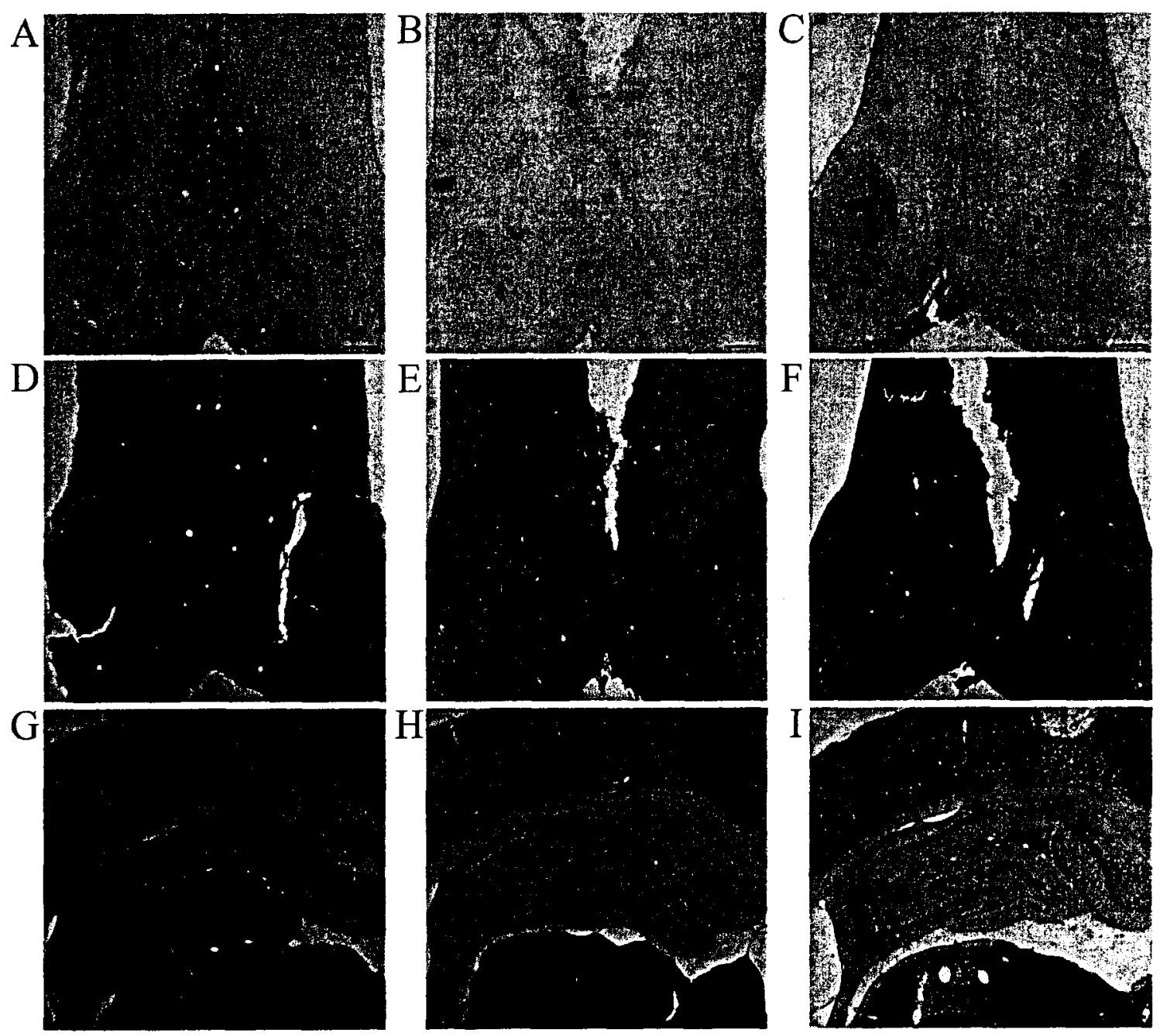

Figure 5.3: Photomicrographs of anti-ChAT labeling in the MS/vDB from a Control rat (A), an AC rat (B) and an AC-stroke rat (C) confirm the near total loss of cholinergic neurons in this area following infusion of IgG SAP. Anti-parvalbumin labeling in the MS/vDB from a Control rat (D), an $\mathrm{AC}$ rat (E) and an AC-stroke rat (F) confirm the specificity of this lesion to cholinergic neurons, while sparing GABA-ergic neurons. The normal AChE staining apparent in the dorsal hippocampus of a Control rat $(G)$ is dramatically reduced following cholinergic depletion in the hippocampus of an AC rat (H) and an AC-stroke rat (I). Scale bars, $0.1 \mathrm{~mm}(\mathrm{~A}-\mathrm{F})$ and $0.5 \mathrm{~mm}(\mathrm{G}-\mathrm{I})$. 
Table 5.1: The number of cholinergic neurons counted in two sections through the medial septum

\begin{tabular}{lccc}
\hline Group & \multicolumn{3}{c}{ Number of ChAT positive neurons } \\
\cline { 2 - 4 } & Section 1 & Section 2 & Total \\
\hline Control & $125.00+/-12.29$ & $129.71+/-19.06$ & $254.71+/-25.99$ \\
\hline AC & $54.33+/-18.53$ & $44.67+/-22.58$ & $99.00+/-31.58$ \\
\hline Stroke & $150.125+/-17.76$ & $158.25+/-24.43$ & $308.38+/-37.27$ \\
\hline AC-stroke & $42.43+/-8.74$ & $18.29+/-6.69$ & $60.71+/-14.52$ \\
\hline
\end{tabular}

Data are given as means $+/-$ SEM.

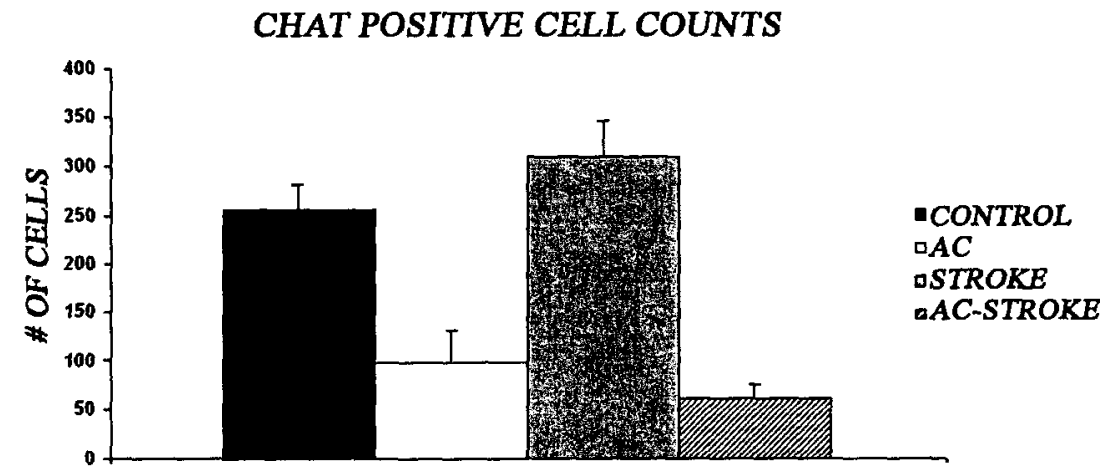

Figure 5.4: Choline acetyltransferase (ChAT) positive cell counts show a clear depletion in $\mathrm{AC}(67 \%)$ and $\mathrm{AC}$-stroke (80\%) rats compared to Stroke and Control rats. Data are given as means $+/$ - SEM.

I did notice that lesioned animals had areas of necrotic cell death in the MS/VDB surrounding the injection site. This non-specific damage has been reported previously (Heckers et al., 1994; Frick et al., 2004) and work in our laboratory has shown no correlation between lesion size (due to focal necrosis following infusion of the toxin) and water maze performance (data not shown). In the current study, a researcher, blinded to 
group conditions, subjectively described the non-specific damage as none, small, medium or large. There was slightly more necrotic damage in $\mathrm{AC}$-stroke (4 large and 3 medium) compared to AC (1 large and 5 medium). I re-analyzed the water maze data with the groups divided by lesion size (AC-stroke-medium, AC-stroke-large and AC-medium; AC-large was not included in the analysis as there was only one rat in this group) to determine whether the larger lesion was responsible for the effect. AC-stroke rats were still impaired on all stages of the water maze task regardless of the amount of nonspecific damage (data not shown).

\section{Quantification of stroke damage in the hippocampus}

Both AC-stroke and Stroke rats had significantly more hippocampal damage compared to Control and AC rats. Damage was localized around the infusion sites in both dorsal (Figure 5.5A-C) and ventral hippocampus (5.5D-F). This damage affected all regions of the hippocampus (CA1, CA2, CA3 and dentate gyrus) and included thinning of cell layers, reactive gliosis and a complete loss of tissue in some regions especially surrounding the needle tract. Some of the Control and $\mathrm{AC}$ rats had minimal disruption of cell layers surrounding the needle tract from the sham infusion procedure, but little damage. In addition to the hippocampal damage, both groups that received the intrahippocampal strokes had some collateral damage in adjacent thalamic nuclei immediately below the dorsal hippocampus. In the Stroke group 7 of the 8 subjects had some bilateral damage to the lateral dorsal thalamic or the medial dorsal nuclei. The other subject in this group had unilateral damage to the lateral dorsal site. In the AC-stroke group, 3 of the 8 subjects had bilateral damage to either the lateral dorsal thalamic or medial dorsal nuclei. Four of the subjects from this group had unilateral damage to the same site and one 
subject had no thalamic damage. Since the damage to these areas was similar or larger in the Stroke group it is very unlikely that this extrahippocampal damage accounts for the enhanced deficit in the AC-stroke group.
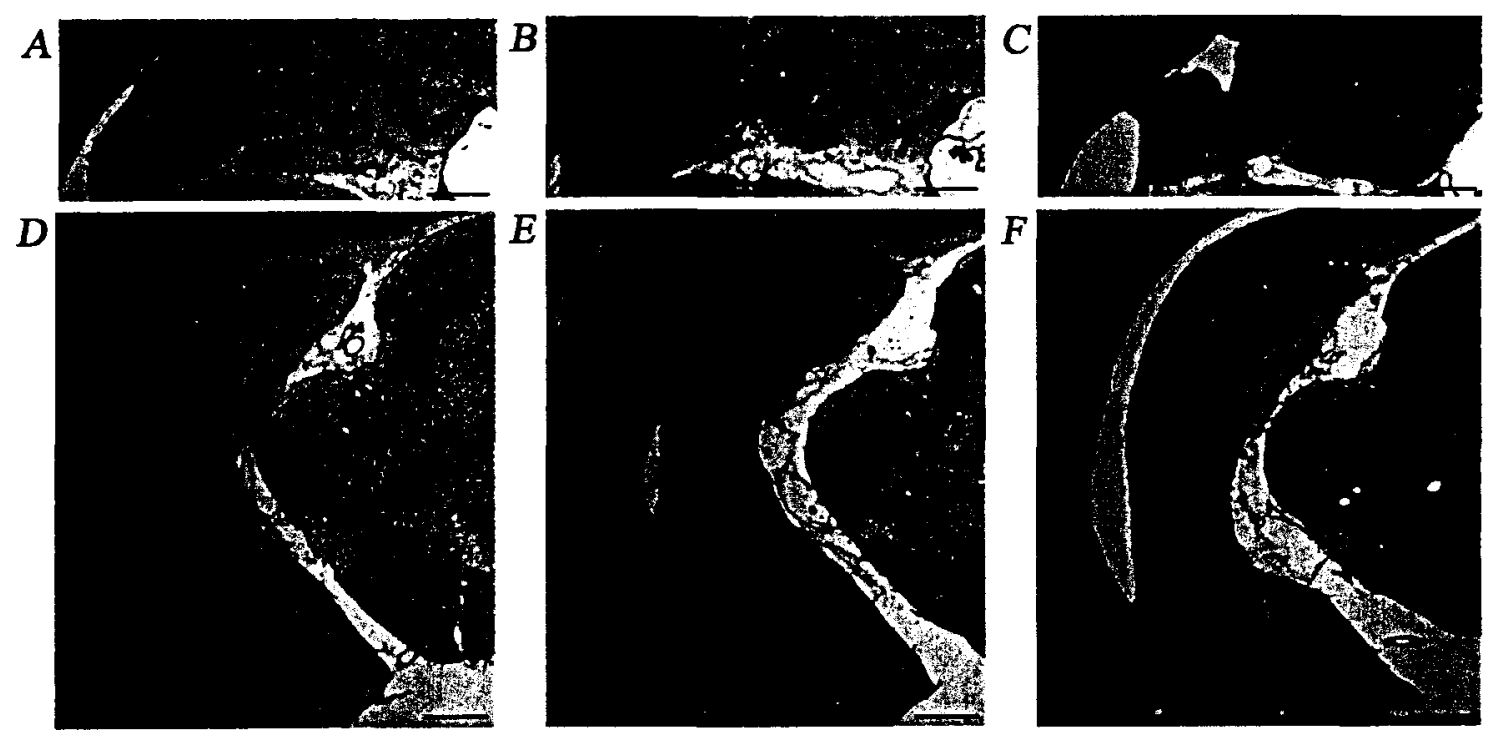

Figure 5.5: Photomicrographs $(5 \mathrm{x})$ of Cresyl violet stained hippocampal sections from a Control rat $(\mathrm{A}, \mathrm{D})$, a Stroke rat $(\mathrm{B}, \mathrm{E})$ and an $\mathrm{AC}$-stroke rat $(\mathrm{C}, \mathrm{F})$. Damage is apparent in both dorsal (A-C) and ventral (D-F) hippocampal sections in the area surrounding the injection site as a result of stroke. Scale bars, $0.5 \mathrm{~mm}$.

The amount of damage was quantified using volumetric analysis (Figure 5.6).

Both Stroke rats and AC-stroke rats had similar amounts of damage $(2919.08+/-845.81$ $\mathrm{mm}^{2}$ versus $3156.61+/-981.33 \mathrm{~mm}^{2}$ ). A one way ANOVA was performed on Lesion size, with Group (AC-stroke versus AC versus Stroke versus Control) as the between subjects variable and revealed a significant effect of group $(F(3,24)=7.00, p<0.01)$.

Holm-Bonferroni post hocs found that the size of the AC-stroke and Stroke lesions were not significantly different, while the lesion size for both these groups was significantly greater than either $\mathrm{AC}$ or Control rats $\left(p^{\prime} s<0.05\right)$. 


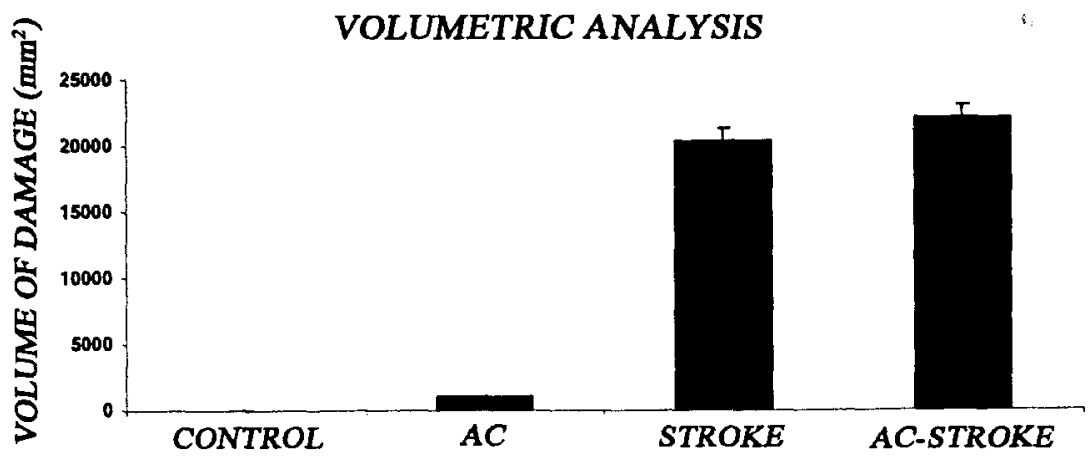

Figure 5.6: Volumetric analysis of damage throughout the entire hippocampal revealed significantly more damage in Stroke and AC-stroke rats compared to Control or AC rats. Data are given as means $+/$ - SEM.

\section{Discussion}

In this study, I have found that a reduction in hippocampal cholinergic activity prior to a small focal stroke resulted in significant impairment in spatial learning. Although stroke on its own did cause mild impairment on a single measure (latency to platform) during original location training on the water maze task, the performance of rats following the combination of cholinergic depletion with stroke was significantly worse on all stages of the water maze task. The volume of hippocampal damage as a result of the stroke was comparable in both groups suggesting it was an interaction between the factors rather than an increase in lesion size that led to the observed behavioural impairment.

There have been a number of studies describing a role for cholinergic agonists in promoting recovery from ischemic or excitotoxic neuronal damage (Yamaguchi et al., 1995; Akaike et al., 2003; Takada et al., 2003; Fujiki et al., 2005; Gonzales et al., 2006). However, damaging basal forebrain cholinergic neurons can lead to reductions in cerebral blood flow (Waite et al., 1999), and cerebral hypoperfusion has been found to be 
protective against focal ischemia (Choi et al., 2007; Kim et al., 2008). These findings suggest a role for the cholinergic system in mediating the brain's response to ischemia and stroke, although a detailed understanding of the underlying mechanism remains unknown. To the best of my knowledge, I am the first to examine the behavioural and gross histological effects of stroke following cholinergic depletion in the rat. The observed impairment of the combined group on all stages of the hidden platform version of the water maze task revealed an interaction between these two factors suggesting a deficit in hippocampal processing. The combined group was able to learn the visible platform version of the water maze (a form of learning that does not require hippocampal function) at control levels demonstrating that they were motivated to escape from the water and that there were no general performance deficits that could explain the impaired learning of the hidden platform task. The enhanced performance of the stroke and depleted only rats on this task was surprising, but hippocampal damage has been shown to bias the selection of a cue strategy over a place strategy (McDonald and White, 1994) which could explain the enhanced performance of my stroke rats. Janis et al. (1998) reported that following cholinergic depletion rats preferred an egocentric cue based strategy, but this finding has been challenged by Bizon et al. (2003) who found that cholinergic depletion of the hippocampus actually biased the rats towards a spatial strategy. My results appear to support Janis et al. (1998), but the enhanced performance of the cholinergic depleted rats in the current study could also be due to their faster swim speeds during visible platform training.

I predicted that in the absence of cholinergic projections to the hippocampus I would see an increase in the amount of damage resulting from stroke. Surprisingly, I 
found no difference in lesion size due to stroke when I compared my sham operated rats to my cholinergic depleted rats. My results are comparable to a previous study in gerbils where Gabriel et al. (1998) electrolytically damaged the medial septum prior to inducing transient global ischemia and reported no change in the extent of hippocampal damage in sham compared to lesioned animals. The appearance of a behavioural deficit in the absence of an increase in lesion size suggests that a more subtle mechanism is responsible for the cognitive impairment in my rats. While there are many studies that have correlated the amount of brain damage with the extent of behavioural impairment (Kiyota et al., 1991; Nedelmann et al., 2007), there have also been a significant number of studies reporting a behavioural effect in the absence of increased damage (Jaspers et al., 1990; Walh et al., 1991; Yamaguchi et al., 1995). The observation that performance is not always correlated with the amount of damage stresses the importance of using both histological and behavioural measures when assessing impairments arising from stroke.

\section{Caveats}

I believe that my overall conclusions are valid and that these findings set the stage for further research into this area; however, there were a few minor caveats associated with this study. Firstly, the amount of non-specific damage to the medial septum following the cholinergic lesion was unexpected; however, this has been reported previously by other groups using interparenchymal infusions of IgG SAP (Heckers et al., 1994; Frick et al., 2004). Moreover, I did not notice any correlation between the extent of the medial septal damage and performance on the water maze task in the lesion only or combined groups (L.Craig, unpublished observations). Previous studies, using less specific methods to damage the medial septum, reported extensive memory impairments 
on spatial memory tasks, similar to the water maze task used here (Marston et al., 1993;

Waite and Thal, 1996). In the current study, lesioned rats were unimpaired on both versions of the water maze task confirming that the non-specific damage observed here was not functionally relevant. Rather, it was the additive effect of the stroke combined with the depletion that resulted in the spatial learning impairment in my combined group. Secondly, the extent of the cholinergic cell loss was less than expected. Previous studies have reported a $90-100 \%$ loss of cholinergic neurons of the medial septum (Baxter et al., 1995; Frick et al., 2004; Craig et al., 2008). Nevertheless, the cell loss seen here is greater than the loss of cholinergic neurons observed during natural aging in humans (Perry et al., 1992) or in rats (Fischer et al., 1991).

\section{Potential mechanisms of functional impairment in subjects following cholinergic depletion and stroke}

The cholinergic system plays an important role in many forms of neuronal plasticity such as functional recovery following injury (Gonzales et al., 2006), neurogenesis (Mohapel et al., 2004; Cooper-Kuhn et al., 2005), and regulating the secretion of neurotrophins such as nerve growth factor (NGF) and brain derived neurotrophic factor (BDNF) (Knipper et al., 1994; French et al., 1999). In the absence of sufficient levels of ACh any one of these mechanisms could potentially have detrimental effects on the hippocampus over time. In combination with a secondary insult such as stroke, low levels of $\mathrm{ACh}$ would leave the hippocampus with a reduced ability to compensate for the damage. I have previously suggested that a cholinergic lesion of the medial septum renders hippocampal neurons increasingly vulnerable to secondary insults. This has been supported in our laboratory in the current study, and by experiments 
demonstrating impairment in spatial memory following a medial septal cholinergic lesion combined with chronic stress (Craig et al., 2008) or when combined with non-convulsive seizures (Craig et al., 2008). I believe that the reduction in ACh does not directly damage hippocampal neurons, but instead acts to increase hippocampal vulnerability to future insults by reducing the brain's ability to compensate for damage.

\section{Potential mechanisms: alterations in plasticity related to reorganization of neuronal} tissue

One possible mechanism to explain my results is the cholinergic system's role in mediating functional recovery following injury. This form of plasticity refers to the reorganization of neuronal tissue in order to compensate for damaged areas. Cholinergic agonists such as nicotine have been found to promote these plastic changes and enhance functional recovery following stroke (Gonzales et al., 2006). Zenardi et al. (2007) reported that transgenic mice lacking high affinity nicotinic receptors are more susceptible to excitotoxic damage, and do not benefit from enriched environment. The neuroprotective role of the cholinergic system is further supported by reports showing the cholinergic agonists, nicotine and donepezil are effective at enhancing recovery and reducing infarct size following stroke in rats (Fujiki et al., 2005; Gonzalez et al., 2006), as well as protecting hippocampal neurons following systemic kainic acid (Borlongan et al., 1995). In addition, pre-treatment with either nicotine or donepezil have been found to protect both cortical and hippocampal neurons from excitotoxic damage in vitro (Akaike et al., 1994; Dajas-Bailador et al., 2000; Takada et al., 2003). Taken together these studies suggest a role for the cholinergic system in mediating recovery from ischemic damage. In the absence of a functional cholinergic system the hippocampus would be less 
able to recover from injury. Cholinergic projections can mediate hippocampal plasticity in two ways: through changes in rates of neurogenesis and in levels of neurotrophin factors essential for neuronal plasticity.

Potential mechanisms: neurogenesis and growth factors

Stroke has been shown to increase levels of neurogenesis (Arvidsson et al., 2001; Jin et al., 2001) and Nakatomi et al. (2002) has found that infusing growth factors [epidermal growth factor (EGF) and fibroblast growth factor (FGF)] intraventricularly following ischemia can enhance the production of new neurons in the rat hippocampus leading to functional recovery. This implies a role for neurogenesis in the recovery from stroke, and stresses the importance of endogenous growth factors. The cholinergic system plays an important role in both neurogenesis and the production of these trophic factors. Following specific cholinergic lesions of the basal forebrain, levels of hippocampal neurogenesis are reduced (Mohepel et al., 2004; Cooper-Kuhn et al., 2005). Moreover, there are reductions in the levels of brain derived neurotrophic factor (BDNF) and nerve growth factor (NGF) available to the hippocampus (Knipper et al., 1994; French et al., 1999). Cholinergic agonists have been shown to increase the production of FGF-2, NGF and BDNF in cortex and hippocampus which could play a role in enhancing neuronal proliferation and/or survival (Belluardo et al., 1998; Maggio et al., 1998; Kenny et al., 2000; Martinez-Rodriguez et al., 2003; Mudo et al., 2007).

\section{Multiple combinations of co-factors}

By understanding the risk factors that have been linked to $\mathrm{AD}$ and the role they play in the development of this disorder, the chances of developing effective treatment strategies are increased. I have begun to look at the effects of multiple risk factors on 
hippocampal damage and measures of learning and memory. Driscoll et al. (2007)

subjected aged rats to a mini-stroke and found increased lesion size as well as significantly worse performance on the spatial water maze. McDonald et al. (2007) reported that when a mini-stroke was given to chronically stressed rats, there was a similar increase in lesion size, but a milder behavioural deficit was observed. I have also shown that by specifically damaging the cholinergic input to the hippocampus, rats are more sensitive to the effects of low doses of kainic acid and to chronic stress, demonstrating impaired water maze performance (Craig et al., 2008; L.Craig, unpublished observations). Taken together, these studies show that the presence of two risk factors that on their own have little effect on memory, when combined can result in significant impairment. Interestingly the extent of the behavioural impairments vary, from mild to severe, depending on which risk factors are present, lending further support to this theory as an accurate model of the differences observed between individuals in the quality of their cognitive aging.

\section{Conclusion}

In conclusion, I have shown that the interaction of these two risk factors (cholinergic depletion and stroke) can lead to impairment in hippocampal processing in the absence of any noticeable changes in the amount of observed damage. I believe this is the result of a reduced plasticity and inability of the hippocampus (in the absence of sufficient $\mathrm{ACh}$ ) to compensate for the damage resulting from an active factor such as stroke. Future studies will look at the possible mechanisms behind this effect in order to open up potential treatment or prevention strategies tailored to those at risk for $\mathrm{AD}$ combined with vascular changes. 


\section{Chapter 6}

Emergence of spatial impairment in rats following specific cholinergic depletion of the medial septum combined with chronic stress

Modified from a paper published in European Journal of Neuroscience, 27, 2262-2271

by Laura A. Craig, Nancy S. Hong, Joelle Kopp and Robert J. McDonald in 2008 


\begin{abstract}
A consistent finding in patients suffering from Alzheimer's disease is a loss of the cholinergic neurons of the basal forebrain that project to the hippocampus. However, the role this depletion plays in the development of Alzheimer's disease remains unclear. The loss of this ascending neurotransmitter system could potentially render hippocampal neurons more susceptible to further insult, such as chronic stress, ultimately resulting in neuronal death and memory loss. I explored this possibility by using the highly specific toxin 192 IgG Saporin to destroy the majority of cholinergic activity in the septohippocampal pathway in rats. Following depletion, rats were subjected to two weeks of restraint stress. Rats were divided into two groups and were tested either on a hippocampus dependent (water maze) task or on a hippocampus independent task (fear conditioning to tone and context). I showed that cholinergic depletion or stress alone had no effect on the successful performance of either of the tasks. However, rats with a combination of cholinergic depletion and stress were significantly impaired on the water maze task. No deficits were apparent in the combined group that was tested on fear conditioning to tone or context, suggesting that this impairment is specific to spatial working memory. These rats had no obvious hippocampal neuronal loss or damage; however there were likely subtle changes in hippocampal processing that led to the observed deficit on the hippocampal dependent task. These findings support my theory that cholinergic depletion of the medial septum increases hippocampal vulnerability to further insults such as stress.
\end{abstract}




\section{Introduction}

Alzheimer's disease (AD) is the leading cause of dementia in the elderly population today (Mesulam, 2004). While the exact cause of $\mathrm{AD}$ is unknown, there have been a number of factors identified as potentially increasing the risk of developing this disorder. McDonald (2002) proposed an etiological model based on the theory that AD pathology originates in the hippocampus (Hyman et al., 1984; Ball et al., 1985). This model classifies risk factors for $\mathrm{AD}$ as either passive factors, which can increase the vulnerability of hippocampal neurons, or active factors, that can directly disrupt learning and memory, through the death or dysfunction of hippocampal neurons.

One of the passive risk factors most often found in $\mathrm{AD}$ patients is a loss of the cholinergic neurons of the basal forebrain that project to the cortex and hippocampus (Davies and Maloney, 1976; Mesulam, 2004). A moderate decline in cholinergic activity is commonly found during the natural aging process of both rats and humans (McGeer et al., 1984; Fischer et al., 1991; Baskerville et al., 2006). This natural cholinergic depletion may affect elderly individuals by increasing hippocampal vulnerability to future insults such as stress, stroke or seizures that could lead to cognitive decline.

Another common passive risk factor that has been linked to $\mathrm{AD}$ is chronic stress (Wilson et al., 2006). Elevated levels of glucocorticoids (corticosterone (CORT) in rats and cortisol in humans) can impair learning and memory in both aged rats (Montaron et al., 2006) and humans (Lupien et al., 1998). Furthermore, prolonged exposure to elevated glucocorticoids can result in hippocampal atrophy and cell death (Magarinos and McEwen, 1995; Sapolsky, 2000). Interestingly, elevated cortisol levels have been found in $\mathrm{AD}$ patients; with higher levels correlating with more severe dementia and increased 
hippocampal atrophy (Davis et al., 1986; Csernansky et al., 2006). This suggests a role for chronic stress and prolonged exposure to glucocorticoids in determining the quality of cognitive aging.

Both cholinergic depletion of the medial septum and stress specifically target the hippocampus. Following a lesion of cholinergic neurons, there are no changes in baseline CORT levels or habituation to chronic stress (Helm et al., 2004); however, there are reductions in hippocampal glucocorticoid receptors (Han et al., 2002) and a heightened CORT response to acute stress (Helm et al., 2004). These alterations suggest a compromised ability to respond appropriately to stress in the absence of a functional cholinergic system. This could lead to increases in hippocampal damage and/or impairment over that which is observed with comparable levels of stress alone.

In the current study, I chose to explore the behavioural consequences of combining cholinergic depletion of the medial septum with a stress procedure designed to chronically elevate CORT levels. I tested rats on either a hippocampal dependent task or on a hippocampal independent task. I predicted that rats receiving either factor alone would perform at control levels on both tasks, while the combination of factors would produce a hippocampal specific deficit, based on my hypothesis that passive factors (such as cholinergic depletion) can increase the vulnerability of hippocampal neurons.

\section{Experiment 1: The effects of cholinergic depletion and chronic stress on levels of blood borne CORT}

My first experiment was designed to ensure that my chronic stress procedure was able to effectively raise CORT levels. I was also interested in whether cholinergic 
depletion of the medial septum would have any effect on overall CORT levels measured following two weeks of chronic stress.

\subsection{Materials and Methods}

Animals

Twenty four male Long Evans hooded rats (300-400g; CCBN breeding colony) were used for this experiment. They were divided into three groups: AC-stress $(n=8)$ received a cholinergic depletion followed by 14 days of stress (these rats were also used in Experiment 3 for behavioural testing), Stress $(n=7)$ received 14 days of stress and Control $(n=8)$ were handled only (Stress and Control rats were from the previous pilot experiment and included for comparison purposes only). Animals were pair housed with food and water ad libitum, on a 12 hour light/dark cycle with lights off at 19:30. All animals were handled in accordance to guidelines set out by the Canadian Council for Animal Care.

Surgery

Surgery was performed under Isoflurane anaesthesia $(4 \%$ with $2.0 \mathrm{~L} / \mathrm{min}$ of oxygen for induction and $2 \%$ after surgical plane was established) in a standard stereotaxic apparatus. An incision was made in the scalp and periosteum down the midline. The fascia was pushed to the edges of the skull with a sterile gauze swab and the skin retracted to expose the skull surface. Holes were drilled into the skull using a 1 $\mathrm{mm}$ drill bit attached to a high speed drill. Following surgery all animals were given buprenorphine (Temgesic; $0.1 \mathrm{mg} / \mathrm{kg}$ subcutaneously) as an analgesic. 


\section{Cholinergic depletion}

The specific neurotoxin 192 IgG Saporin (IgG SAP; Chemicon) has been commonly used to induce selective cholinergic lesions in rats depending on the site of infusion. I chose to damage cholinergic neurons in the medial septum and vertical limb of the diagonal band of Broca (MS/VDB) which leads to a reduction in levels of hippocampal acetylcholinesterase (AChE). I lowered a 30 gauge cannula, through holes drilled in the skull, into the MS/VDB (AP +0.45; ML +/-0.6; DV -6.6 and $-8.0 \mathrm{~mm}$ from bregma and the skull) and bilaterally infused IgG SAP $[0.15 \mu \mathrm{g} / \mu 1$ dissolved in sterile $1 \mathrm{x}$ phosphate buffer saline (PBS; $\mathrm{pH} 7.4$ )] at a rate of $0.05 \mu 1 / \mathrm{min}$ using a Harvard minipump. Each animal received a total volume of $0.3 \mu \mathrm{l}$ per side at the first injection site (DV -8.0), and $0.2 \mu 1$ per side at the second site (DV -6.6). The cannula was left in place for six and four minutes after the first and second injection respectively to ensure diffusion of the toxin.

\section{Variable restraint stress}

I used a variable restraint stress schedule to increase CORT levels over a two week period. This schedule was designed to decrease the rats' ability to acclimatize to the stress duration and minimize habituation. To induce stress, rats were taken in their home cages to a testing room between 11:30 - 13:30 each day and restrained in Plexiglas cylinders (diameter $6.5 \mathrm{~cm}$, length $15 \mathrm{~cm})$ for a variable amount of time each day $(20,5$, $60,15,10,30$ or 40 minutes). This cycle was repeated twice for a total of 14 days of stress.

Blood collection and analysis of CORT levels: To assess levels of circulating CORT, rats were placed in a restraint tube and blood samples were obtained by tail nick with a 
scalpel blade on the final day of stress. Blood was collected from AC-stress and Stress rats 30 minutes after being placed in the restraint tube to obtain a peak level of CORT in response to the stressor. Blood was collected from Control rats within the first three minutes of being placed in the restraint tube to ensure CORT levels did not have time to significantly increase in response to the brief stress of the procedure. All blood samples $(0.2-0.5 \mathrm{ml})$ were collected into tubes containing $100 \mu \mathrm{l}$ of $10 \%(\mathrm{v} / \mathrm{v})$ ethylenediaminetetraacetic acid (EDTA), placed on ice, centrifuged (7500 rpms for 10 minutes) and the plasma was immediately frozen at $-20^{\circ} \mathrm{C}$. A commercially available ${ }^{125} \mathrm{I}$ radioimmunoassay kit (Coat-A-Count Rat Corticosterone, DPC, California) was used for quantifying levels of plasma CORT for each animal.

\subsection{Results}

\section{Analysis of blood borne CORT levels}

To confirm that my stress procedure produced elevated glucocorticoid levels, I took blood samples on the final day of stress and analyzed them for levels of blood borne CORT. Both AC-stress and Stress rats showed a significant elevation in CORT levels following 14 days of variable restraint stress when compared to Control rats (Figure 6.1). There was no difference between AC-stress and Stress rats in overall CORT levels $(308.73+/-38.75 \mathrm{ng} / \mathrm{ml}$ and $279.18+/-29.62 \mathrm{ng} / \mathrm{ml}$ respectively). Data was analyzed using a one way analysis of variance (ANOVA) that found an effect of group $[F(2,21)=32.99, p<0.01]$. Tukey post hocs revealed that AC-stress and Stress rats both had increased levels of CORT compared to Control rats $(p<0.01)$. 


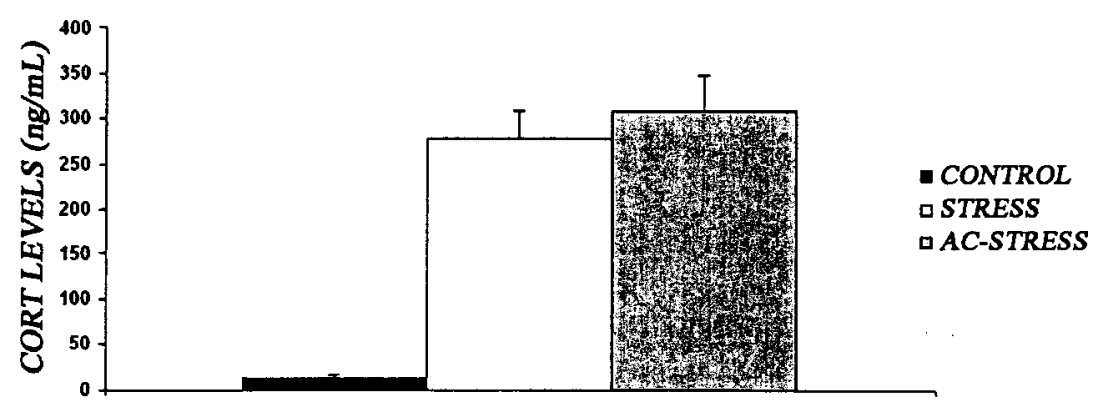

Figure 6.1: AC-stress and Stress rats have elevated levels of CORT compared to Control rats on the final day of the 14 day variable stress procedure. Data are given as means $+/-$ SEM.

\section{Experiment 2: The effects of cholinergic depletion and chronic stress on the} performance of a hippocampal dependent task.

My second experiment was designed to determine the effects of cholinergic depletion of the medial septum followed by chronic stress on the ability of the rat to complete a hippocampal dependent task (water maze).

\subsection{Materials and Methods}

\section{Animals}

Thirty two male Long Evans hooded rats (300-400g; CCBN breeding colony) were used for this experiment. They were divided into four groups: AC-stress $(n=8)$ received a cholinergic depletion followed by 14 days of stress, $\mathrm{AC}(n=8)$ received a cholinergic depletion followed by 14 days of handling"only, Stress $(n=8)$ received a sham depletion followed by 14 days of stress and Control $(n=8)$ were handled only. All animals were housed identically to conditions described in Experiment 1. 


\section{Surgery}

The surgical procedures were identical to those described in Experiment 1. Sham operated animals underwent the same procedure, but were infused with sterile 1x PBS instead of IgG SAP.

\section{Variable restraint stress}

The variable stress procedure was identical to the one described in Experiment 1.

\section{Behavioural testing}

Behavioural testing began 24 hours following the final day of stress.

\section{Water maze: Rapid Acquisition Task}

I used a three stage variant of the spatial version of the water maze task (McDonald et al., 2005). A white plastic circular pool $1.4 \mathrm{~m}$ in diameter and $40 \mathrm{~cm}$ deep was filled to within $20 \mathrm{~cm}$ of the top of the wall with water $\left(20-22^{\circ} \mathrm{C}\right)$ made opaque by adding skim milk powder. The invisible platform was $12 \mathrm{~cm}$ in diameter and made of white Plexiglas with holes drilled into the top of it to provide grip for the animals. During training the platform was submerged $2 \mathrm{~cm}$ under water. Extra-maze cues in the training room included five posters of different sizes and orientations mounted on three of the four walls, a computer rack, a door, an animal rack and the experimenter. Four different start points were used [N, S, E, W (not true compass headings)], equally spaced around the pool. The order was randomly selected for each day and each start point was used twice for day one to four and day six. On day five only the three start points farthest from the platform were used (N, E, S) with the initial start point repeated at the end for a total of 16 trials. 
In the first stage (Original location training) rats were trained over four days for eight trials a day, to swim to a fixed, spatial location. Twenty four hours after completion of stage one; the second stage (New location training) began. This stage consisted of new spatial location training as animals were re-trained to swim to the new platform location over sixteen trials within two hours on a single day. Stage two allowed me to ask if rats could learn to go to a new location, during a single intensive training session. The third stage (Re-training to original location) occurred twenty four hours after stage two with the platform returned to its original (stage one) location and each animal was given eight trials. This stage allowed me to ask how the massed new location training during stage two affected relearning to the original location.

This version of the water maze was selected due to its sensitivity to hippocampal damage. The first stage allowed me to identify impairment in acquisition and short term memory of an invariant location. If no differences were apparent, the second stage would test the ability of the rat to rapidly form a novel representation in a familiar context. For the third stage, the subjects' are required to re-learn the original location (McDonald et al., 2005).

Data collection: A computer based rat tracker (VP118, HVS Image) was used to collect and analyze data obtained from an overhead video camera. If a rat did not reach the platform 60 seconds after release, the rat was guided manually to the platform (aided placement) and allowed to remain on the platform for 10 seconds before being removed to a holding cage until the next trial. For all three stages the measures of performance were latency to platform and path length to platform. Path length is a sensitive measure that can control for potential differences in swim speed. I also recorded the number of 
aided placements for each animal and used this measure to confirm that rats were actively searching for the platform as opposed to 'floating' or being excessively thigmotaxic.

\subsection{Results}

One rat assigned to the Stress group died post surgery; the following analysis includes data from seven rats in this group.

\section{Water maze: Rapid Acquisition Task}

\section{Original location training}

The latency (in seconds) to find the platform was recorded and averaged over the eight daily trials to obtain a single daily path length for each animal (Figure 6.2A). The AC-stress group was impaired in their ability to learn the platform location during original location training. The daily latencies for all four days were subjected to a twoway mixed ANOVA with Group as the between subjects variable, and Training Day (four levels) as a repeated measure, and revealed a significant effect of group $[F(3,27)=5.47$, $p<0.01]$ and of day $[F(3,81)=94.76, p<0.01]$, but no interaction $[F(9,81)=1.32, p=0.24]$. By the final day of training, the daily latency score for AC-stress rats was almost twice as long as the averaged score for the other three groups $(12.00+/-1.80$ seconds versus 6.07 +/- 1.06 seconds). Holm-Bonferroni post hocs revealed that AC-stress rats were impaired compared to all other groups ( $\left.p^{\prime} s<0.05\right)$.

I also measured the path length (in meters) to find the platform and found a similar pattern of results. The AC-stress group appeared to be impaired in their ability to learn the platform location during original location training, with a path length of $2.17+/-$ 0.43 meters on the final day of training, compared to the averaged path length of $\mathrm{AC}$, Stress and Control groups (1.32 +/- 0.27 meters); however, this trend was not significant 
(6.2B). The daily path lengths for original location training were subjected to a two-way mixed ANOVA with Group (AC-stress versus AC versus Stress versus Control) as the between subjects variable, and Training Day (four levels) as a repeated measure, and revealed a significant effect of day $[F(3,81)=103.91, p<0.01]$, but not of group $[F(3,27)=2.32, p=0.10]$ and no interaction $[F(9,81)=0.87, p=0.55]$. Tukey post hocs revealed that all groups exhibited shorter path lengths from day one $(6.84+/-0.76$ meters) to day four $(1.53+/-0.31$ meters; $p$ 's $<0.05)$.
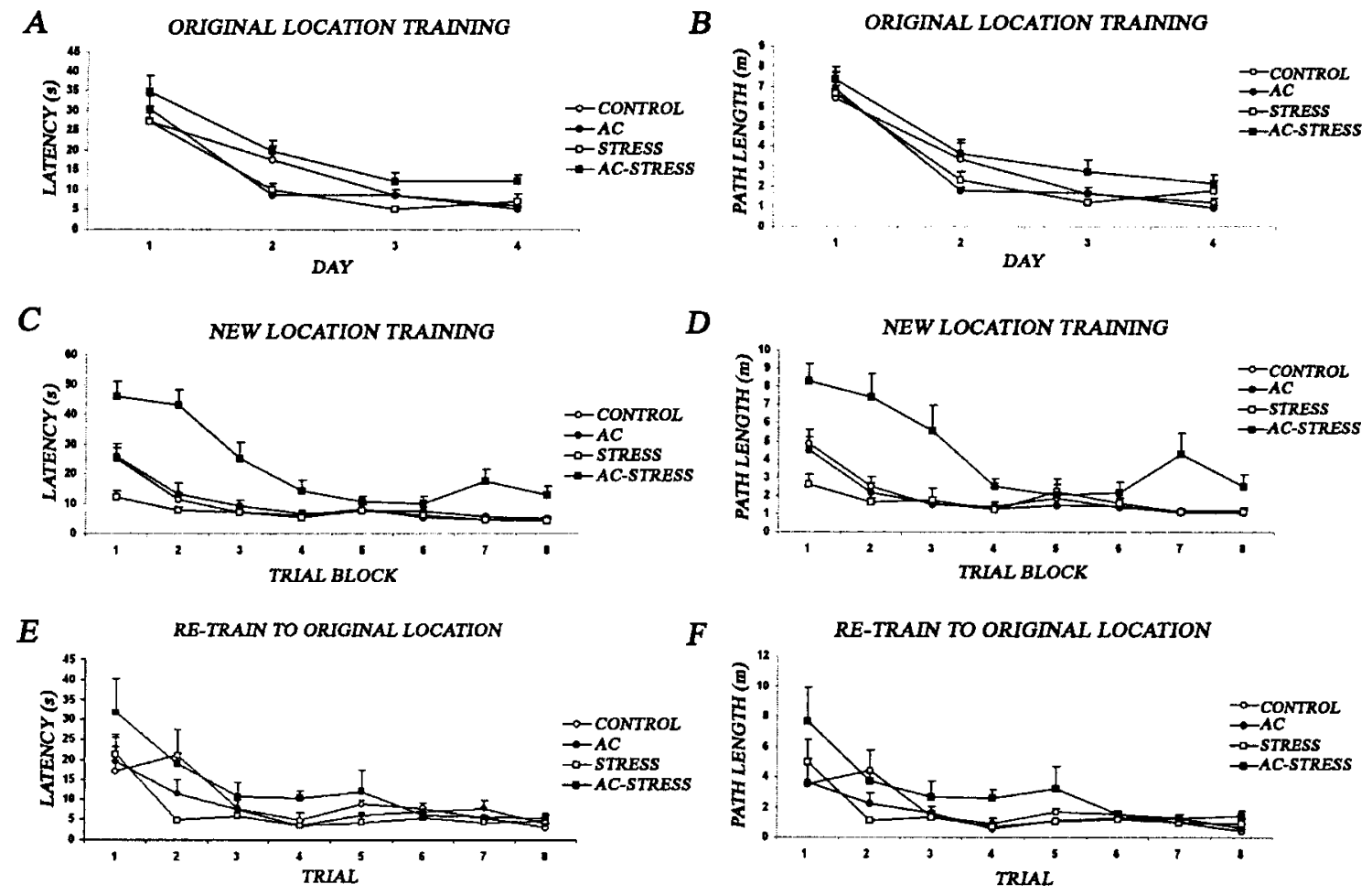

Figure 6.2: AC-stress rats are impaired on all stages of the spatial water maze task. Measures of latency and path length of platform revealed that AC-stress rats were impaired compared to $\mathrm{AC}$, Stress and Control rats on original location training $(\mathrm{A}, \mathrm{B})$, new location training $(C, D)$ and re-training to the original location (E,F). Data are given as means $+/$ - SEM. 
New location training

The platform was moved to the opposite quadrant of the pool and the path length to find the new platform location was recorded and averaged into eight discrete trial blocks (two trials per block). The AC-stress group was impaired in their ability to rapidly acquire a new platform location on this stage of this task when compared to all other groups (6.2D). By the final trial block, AC-stress rats were still taking longer to find the platform (12.96 +/- 2.94 seconds) than the averaged latency of the other three groups (4.63 +/- 0.49 seconds). A two-way mixed ANOVA with Group as a between subject variable and Trial Block (eight levels) as a repeated measure, was used to assess the mass training and found a significant effect of group $[F(3,27)=22.71, p<0.01]$, of trial block $[F(7,189)=36.65, p<0.01]$, and an interaction $[F(21,189)=5.39, p<0.01]$. The interaction was further broken down using planned comparisons that showed significantly longer latencies for AC-stress rats on trial blocks one to four, and seven to eight, compared to all other groups $(p ' s<0.05)$.

I also used path length as a measure of performance and found the same pattern of results. The AC-stress group was impaired in their ability to rapidly acquire a new platform location on the mass training stage of this task when compared to all other groups (Figure 6.2D). By the final trial block, AC-Stress rats were taking longer to find the platform $(4.35+/-0.63$ meters $)$ than the averaged path length of the other three groups (1.82+/- 0.01 meters). A two-way mixed ANOVA with Group as a between subject variable and Trial Block (eight levels) as a repeated measure, was used to assess the mass training and found a significant effect of group $[F(3,27)=16.31, p<0.01]$, of trial block $[F(7,189)=20.47, p<0.01]$, and an interaction $[F(21,189)=3.44, p<0.01]$. The 
interaction was further broken down using planned comparisons that showed significantly longer path lengths for AC-Stress rats on trial blocks one to four, and seven to eight, compared to all other groups ( $p$ 's $<0.05)$.

\section{Re-training to original location}

The platform was returned to the original location (from stage 1) and the latency to platform was recorded over eight trials on the final day of testing. Surprisingly, there were no differences in latency to platform for any of the groups. AC-stress rats were able to learn the original location on this stage of training (6.2E). All groups showed a significant decrease in latency to find the platform from trial one $(22.41+/-6.43$ seconds $)$ to trial eight (4.19+/-1.19 seconds). A two-way mixed ANOVA, with Group as the between subjects variable, and Trial (eight levels) as a repeated measure revealed a significant effect of trial $[F(7,189)=16.62, p<0.01]$, but no effect of group $[F(3,27)=2.49$, $p=0.08]$ or interaction $[F(21,189)=1.26, p=0.21]$.

I also measured the path length to find this location and using this measure found that the AC-stress group was impaired in their ability to learn the original platform location (6.2F). By the final trial AC-stress rats were taking twice as long $(1.40+/-0.38$ meters) as the averaged path length for the other three groups ( $0.66+/-0.26$ meters). A two-way mixed ANOVA, with Group as the between subjects variable, and Trial (eight levels) as a repeated measure revealed a significant effect of group $[F(3,27)=3.95$, $p<0.05]$ and of trial $[F(7,189)=12.87, p<0.01]$, but no interaction $[F(21,189)=1.17$, $p=0.28]$. Holm-Bonferroni post hocs showed AC-stress rats were significantly impaired compared to all other groups $\left(p^{\prime} s<0.05\right)$. 
To further assess the competition data, individual swim paths on the first trial were then analyzed to obtain a measure of the animal's memory for the platform locations. The swim paths differed markedly between groups, with the AC, stress and control animals swimming first to the new location, and then after failing to find the platform, swimming to the original location (Figure 6.3A - C). In contrast, the AC-stress animals appeared to have no clear preference, and swam randomly throughout the pool (6.3D). In order to quantify these data, I chose to use quadrant entry as a categorical measure of platform memory. Each swim path was classed as new, original, or middle depending on which quadrant the rat first entered upon leaving the starting quadrant. These data were then subjected to a binomial test with the categories set as new or other (combined choice of original and middle), with a 0.33 expected probability of entering the new quadrant. The AC, Stress and Control groups showed a clear preference for the new quadrant as seven out of eight $\mathrm{AC}$, and seven out of eight Control and six out of seven Stress rats $(p<0.05)$ entered the new quadrant first (Figure 6.3E). None of the ACstress animals entered the new quadrant first, but six out of eight rats swam directly to the original quadrant $(p<0.05)$. However, a closer inspection of their swim paths revealed that although these rats entered the original quadrant first, suggesting a memory for this location, they were unable to find the platform. Instead, these rats swam randomly throughout the pool until they bumped into the platform and escaped. 

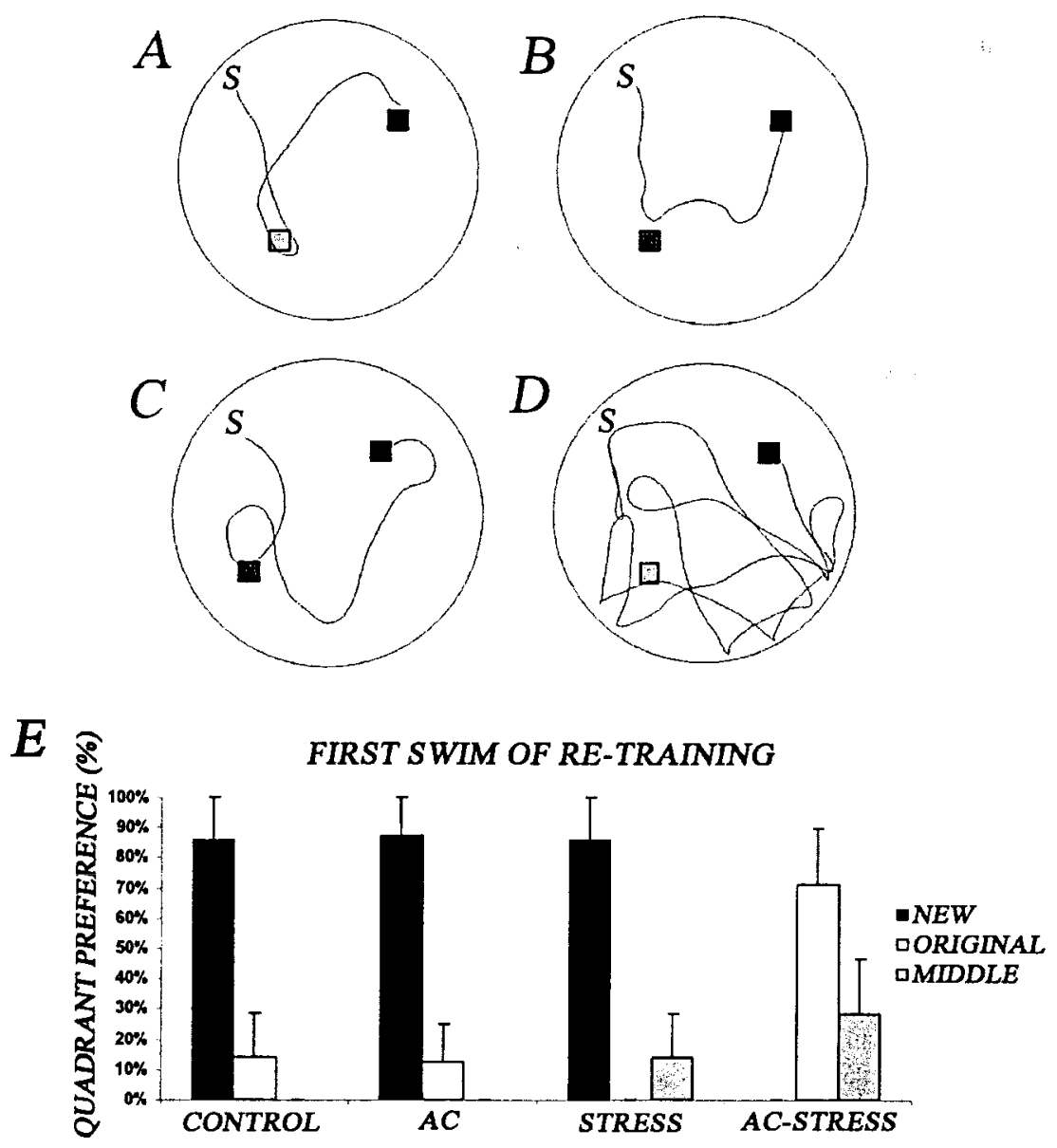

Figure 6.3: The initial swim on the competition day of the spatial water maze was analyzed to determine platform preference. Representative swim paths from each group [Control (A), AC (B), Stress (C) and AC-stress (D)] show that AC-stress rats swam randomly throughout the pool, while all other groups swam first to the new platform, then headed towards the original platform location and escaped from the water $(E)$. Data are given as means $+/-$ SEM.

\section{Aided Placements}

A potential confound when conducting behavioural testing with stressed animals is the possibility of learned helplessness or anxiety related behaviours. In the water maze, learned helplessness is often expressed as 'floating' defined as immobility of the rat with just enough movement to stay afloat (Porsolt et al., 1978), while excessive thigmotaxic 
behaviour is a sign of heightened anxiety (Barnett, 1963; Devan et al., 1999). These behaviours are not conducive to an effective search strategy and often necessitate the experimenter guiding the rat to the platform at the cessation of the trial (aided placement). I predicted that if my stressed rats (AC-stress or Stress) were engaging in these behaviours they would have more aided placements throughout each day of training than Control or $\mathrm{AC}$ rats.

The number of aided placements during original location training were subjected to a two-way mixed ANOVA with Group as the between subjects variable and Training Day (four levels) as the repeated measure. This analysis revealed a significant effect of group $[F(3,27)=3.63, p<0.05]$, day $[F(3,81)=88.92, p<0.001]$ and an interaction $[F(9,81)=2.60, p<0.01]$. The interaction was broken down further using planned comparisons that showed that AC-stress rats had significantly more aided placements ( $42 \%$ of all trials were aided placements) on day one than AC or Stress rats $(31 \%$ and $23 \%$ aided placements respectively; $p$ 's $<0.05$ ). While AC-stress rats did not differ significantly from Control rats $(26 \%$ aided placements; $p=0.08)$ on day one, there was a trend for more aided placements in the combined group. By days three and four there were no differences between any of the groups showing all rats were exploring sufficiently to find the platform within the 60 second time limit (Figure 6.4A).

The number of aided placements during new location training were subjected to a one-way ANOVA and found a significant effect of group $[F(3,27)=11.98, p<0.001]$. Tukey post hocs found that AC-stress rats had more aided placements than the other groups $(p ' s<0.05)$. However, the increased number of aided placements occurred during the first four trial blocks of mass training where AC-stress rats had 14 aided placements 
compared to Control, $\mathrm{AC}$ and Stress groups (two, one and no aided placements respectively). By the last four trial blocks there was only one aided placement in the ACstress group demonstrating these rats were actively searching for the platform, even if their strategy was less efficient (6.4B).

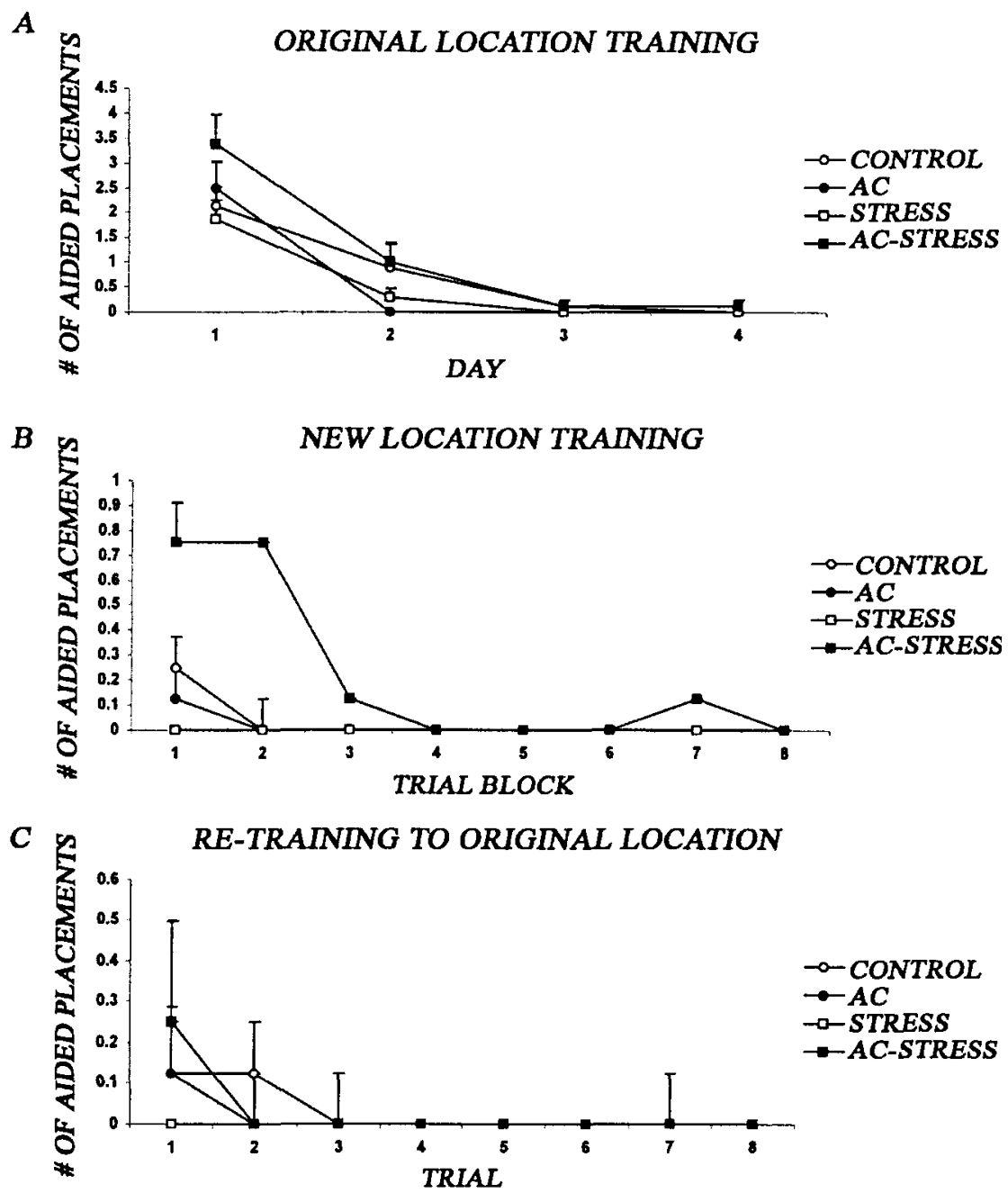

Figure 6.4: To ensure AC-stress rats were actively searching for the platform, I analyzed the number of aided placements. AC-stress rats had a few more aided placements on the initial day of original location training and on the first few trial blocks of new location training, but overall were actively searching for the platform during all three stages of training and had a similar number of placements to all other groups (A-C). Data are given as means $+/$ - SEM. 
There were no differences in the number of aided placements during the retraining to original location stage. A one way ANOVA with fixed effects was performed to detect differences in the total number of aided placements and found no differences between groups $[F(3,27)=0.49, p=0.69](6.4 C)$.

The performance of rats on a hippocampal dependent task (water maze) following cholinergic depletion combined with chronic stress was significantly compromised. All three stages of this task were affected. AC-stress rats had more difficulty locating the platform during original location training and were significantly impaired in their ability to learn a new location in a single day. This group was also impaired during re-training to the original location.

\section{Experiment 3: The effects of cholinergic depletion and chronic stress on a}

\section{hippocampal independent task.}

My third experiment was designed to determine the effects of cholinergic depletion of the medial septum followed by chronic stress on the ability of the rat to complete a hippocampal independent task (fear conditioning).

\subsection{Materials and Methods}

\section{Animals}

Thirty two male Long Evans hooded rats $(300-400 \mathrm{~g})$ were used for Experiment 3. They were divided into four groups: AC-stress $(n=8)$ received a cholinergic depletion followed by 14 days of stress, AC ( $n=8)$ received a cholinergic depletion followed by 14 days of handling only, Stress $(n=8)$ received a sham depletion followed by 14 days of 
stress and Control $(n=8)$ were handled only. All animals were housed identically to conditions described in Experiment 1.

\section{Surgery}

The surgical procedures were identical to those described in Experiment 2.

\section{Variable restraint stress}

The variable stress procedure was identical to the one described in Experiment 1.

\section{Behavioural testing}

Behavioural testing began 24 hours after the final day of stress.

\section{Fear conditioning to tone and context}

The three day fear conditioning procedure used here was designed to test hippocampal independent learning and memory on two related tasks: fear conditioning to tone (cue) and to context. Both cued and contextual fear conditioning are dependent on the amygdala for successful learning (Fanselow and LeDoux, 1999; Ponnusamy et al., 2007). Contextual fear conditioning does have a hippocampal component, but when distinctly different contexts and multiple context-shock pairings are used (as was in the current study) the amygdala is able to successfully form an association without hippocampal involvement (Frankland et al., 1998; Wiltgen et al., 2006).

Animals were placed into Context A (an operant chamber, metal walls, bars on floor, scented with Quatsyl@-D Plus disinfectant) and allowed to habituate for two minutes. They were presented with a 10 second tone $(80 \mathrm{db})$, followed by a two second shock (1.0 mA). This tone-shock pairing was repeated every 70 seconds for five repetitions. Sixty seconds following the final shock, the animals were removed from the 
chamber, and returned to their home cage. Twenty four hours later the animals were placed in a novel context, Context B (Plexiglas walls, bedding on the floor, unscented), and after two minutes of habituation, were presented with an eight minute tone $(80 \mathrm{db})$, with no shock and scored for freezing behaviour, as a measure of fear. Freezing behaviour is a reliable measure of fear, and was defined as a period of complete immobility except for breathing movements in the animal (Blanchard and Blanchard, 1969). Forty eight hours later animals were returned to Context A for a five minute trial (no shock, no tone) to assess freezing behaviour in response to the original conditioning context.

\subsection{Results}

Due to a malfunction of the shock boxes, two AC-stress rats were excluded from this analysis.

\section{Fear conditioning}

\section{Conditioning to tone}

All groups were able to learn the tone-shock association, and showed an increase in freezing behaviour in the presence of the tone. The percentage of freezing during the first four minutes exposed to the tone was calculated and compared to the Context B habituation period. This percentage of freezing for all groups was significantly greater in the presence of the tone $(48.17 \%+/-12.55 \%$ versus $20.48 \%+/-12.55 \%$; Figure $6.5 \mathrm{~A})$. A two-way mixed ANOVA with Group as a between subjects variable, and Tone (habituation versus tone presentation) as a within subjects variable, revealed a significant effect of tone $[F(1,24)=29.61, p<0.001]$, but no group effect $[F(3,24)=1.02, p=0.40]$ or interaction $[F(3,24)=0.77, p=0.52]$. 


\section{Conditioning to context}

All groups were able to learn the context-shock association, and showed an increase in freezing behaviour when returned to Context $\mathrm{A}$ on day three. I measured a percentage freezing for the total time exposed to Context $\mathrm{A}$ on day three and compared it to the Context A habituation on day one. The percentage of freezing to context for all groups was significantly greater on day three compared to day one $(46.55 \%+/-11.96 \%$ versus $2.46 \%+/-0.96 \%$; Figure 6.5B). A two-way mixed ANOVA with Group as a between subjects variable, and Context (day one versus day three) as a within subjects variable, revealed a significant effect of context $[F(1,24)=48.16, p<0.01]$, but no group effect $[F(3,24)=0.47, p=0.70]$ or interaction $[F(3,24)=0.65, p=0.59]$.

Following the combination of cholinergic depletion and chronic stress, rats retained their ability to successfully learn a hippocampal independent task (fear conditioning to tone and context) at a level comparable to that seen in all three control groups. 
$\boldsymbol{A}$

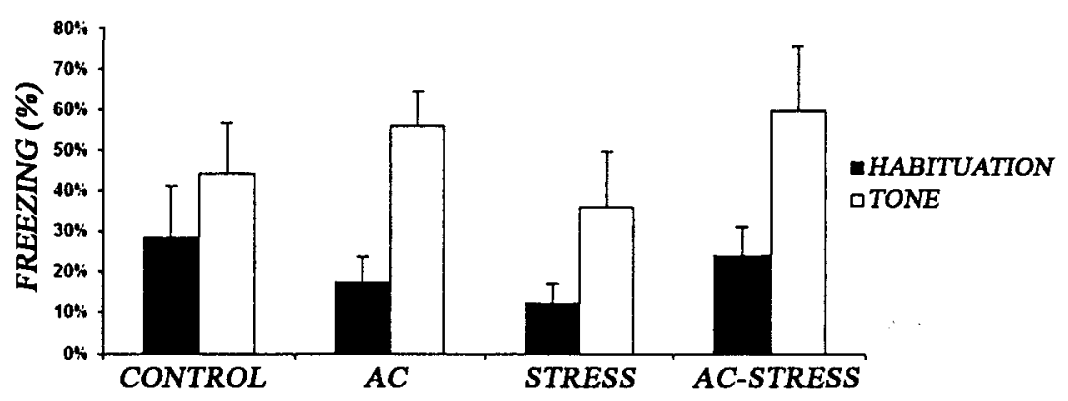

$B$

FEAR CONDITIONING TO CONTEXT

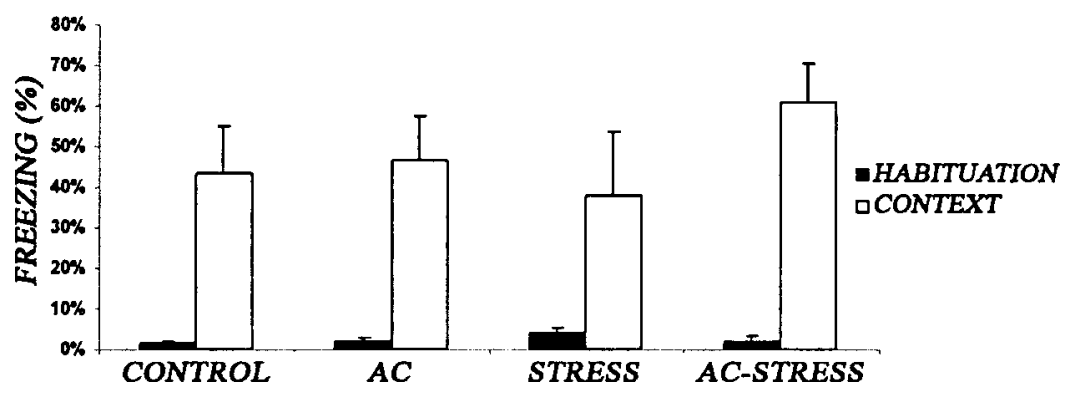

Figure 6.5: Performance on the fear conditioning task. There were no impairments in any of the groups on either fear conditioning to tone (A) or to context (B). Data are given as means +/- SEM.

\section{Histology}

Following behavioural testing all animals were sacrificed with an overdose of Euthanol and transcardially perfused with $1 \times$ PBS followed by $4 \%(w / v)$ paraformaldehyde (PFA). The brains were carefully extracted and placed in $4 \%$ PFA at $4^{\circ} \mathrm{C}$ for 24 hours. Brains were then transferred to a $30 \%(w / v)$ sucrose solution, containing $0.02 \%(\mathrm{w} / \mathrm{v})$ sodium azide at $4^{\circ} \mathrm{C}$ for at least three days. Brains were sectioned on a freezing cryostat at $40 \mu \mathrm{m}$ in a series of five. The first two sections of each series were mounted onto $1 \%(\mathrm{w} / \mathrm{v})$ gelatin $/ 0.2 \%(\mathrm{w} / \mathrm{v})$ chromalum coated slides, while the third, fourth, and fifth series were stored in $1 \mathrm{x}$ PBS containing $0.02 \%(\mathrm{w} / \mathrm{v})$ sodium azide 
at $4^{\circ} \mathrm{C}$ until being processed for immunohistochemistry. The fifth series was not used in this experiment and was stored for future analyses.

\section{Cresyl Violet staining}

The first series was stained with Cresyl violet and visually inspected under a 10x objective to determine if there was any structural damage to the hippocampus.

\section{AChE staining}

The second series was stained for $\mathrm{AChE}$ to get a qualitative image of cholinergic depletion in the hippocampus. I treated the slides for 30 minutes with $6.24 \mathrm{mg}$ tetraisopropylpyrophosphoramide (Sigma) dissolved in $200 \mathrm{~mL}$ of distilled water, then rinsed the slides twice with distilled water and incubated them on a shaker table for four hours in a reaction mixture containing $5.72 \mathrm{~g}$ Trizma maleate, $2.04 \mathrm{~g}$ Trizma base, 100 mg acetylthiocholine iodide, $294 \mathrm{mg}$ dihydrous sodium citrate, $150 \mathrm{mg}$ anhydrous cupric sulphate, $32.8 \mathrm{mg}$ potassium ferricyanide dissolved in $200 \mathrm{~mL}$ distilled water (all chemicals were obtained from Sigma). Slides were rinsed twice with distilled water, and left to dry overnight, then cleared with HemoDe (Sigma) and coverslipped with Permount (Sigma) the next day.

\section{Immunohistochemistry}

The third and fourth series were processed for immunohistochemistry to determine the completeness and specificity of the cholinergic depletion. An antibody raised against parvalbumin was used to label $\gamma$-aminobutyric acid (GABA)-ergic neurons, and one against choline acetyltransferase (ChAT) was used to label cholinergic neurons. Free floating sections were rinsed in $1 x$ PBS, and then placed in a $3 \%(v / v) \mathrm{H}_{2} \mathrm{O}_{2}$ solution for four minutes to block endogenous peroxidases. After three washes in 1x PBS, 
sections were blocked for 30 minutes in 15\% (v/v) normal horse serum (for antiparvalbumin; Vector Labs) or $15 \%$ (v/v) normal rabbit serum (for anti-ChAT; Vector Labs). Sections were incubated overnight in primary antibody [anti-ChAT polyclonal, 1:1000 (Chemicon) or anti-parvalbumin monoclonal, 1:5000 (Sigma)] diluted in 1x PBS with $7.5 \%$ (v/v) serum (horse for anti-parvalbumin; rabbit for anti-ChAT). Sections were then washed three times in 1x PBS and incubated for 30 minutes in a biotinylated secondary antibody (ABC Elite Kit, Vector Labs), washed three times in 1x PBS, incubated for 30 minutes with $\mathrm{AB}$ reagent ( $\mathrm{ABC}$ Elite Kits, Vector Labs), washed three times in 1x PBS then developed with diaminobenzidine (DAB) substrate (Vector Labs) according to manufacturers instructions. Sections were mounted onto $1 \%(\mathrm{w} / \mathrm{v})$ gelatin $/ 0.2 \%(\mathrm{w} / \mathrm{v})$ chromalum coated slides, air dried overnight, then dehydrated in alcohol, cleared in HemoDe (Sigma) and coverslipped with Permount (Sigma).

Cell counts: Under light microscopy (AxioVision) ChAT positive neurons were counted in both hemispheres of the MS/VDB. I chose two sections to quantify; the first, at the site of injection and the second, in a section $200 \mu \mathrm{m}$ caudal to the injection site. This quantification was not used as an accurate count of MS/VDB neurons, but instead to estimate the completeness of the cholinergic depletion. Each hemisphere was counted separately to confirm lesion symmetry, after which counts for both hemispheres were then totaled for a representative cell count over the two sections.

\section{Histological results}

All the cholinergic depleted rats used in these experiments had similar lesions so I grouped them for histological analysis [AC-stress ( $n=16)$, Stress ( $n=15), \mathrm{AC}(n=16)]$. Control rats for the behavioural portions of this study were from a separate experiment 
(unpublished), and I did not have access to the tissue for histological analysis. However, comparisons of the Stress rats used here with control rats from previous work in our laboratory have repeatedly found no difference in ChAT positive cell counts or staining so I have included a separate representative Control group $(n=10)$ in the analysis here for comparative purposes.

\section{Quantification of cholinergic lesion}

I confirmed the completeness and specificity of the cholinergic lesion in both AC and $\mathrm{AC}$-stress group through $\mathrm{AChE}$ staining and immunohistochemistry for ChAT and parvalbumin. Lesioned animals had very few ChAT positive cholinergic neurons (Figure 6.6A,B) combined with intact parvalbumin labeled GABA-ergic neurons confirming the specificity of the lesion to cholinergic neurons (Figure 6.6C,D). The AChE staining clearly showed a depletion of cholinergic activity in the hippocampus in lesioned animals compared to the sham operated groups $(6.6 \mathrm{E}, \mathrm{F})$.

I quantified the extent of the cholinergic lesion by comparing ChAT positive cell counts between groups. I combined the ChAT positive cell counts from the Stress and Control groups, and used this average to determine the percent depletion for the lesioned groups. I found $97 \%$ depletion in the AC-stress group and $98 \%$ depletion in the AC group. An independent samples t-test showed no significant difference between the two groups $[\mathrm{t}(28)=-0.59, p=0.56]$. There were significantly fewer cholinergic neurons in $\mathrm{AC}$ and AC-stress compared to the Stress and Control group (Table 6.1). A one way ANOVA revealed a significant effect of group $[F(3,51)=115.01, p<0.01]$. Tukey post hocs showed that the $\mathrm{AC}$ and $\mathrm{AC}$-stress groups had significantly fewer ChAT positive cells than either of the Stress and Control groups ( $p$ 's $<0.05$; Figure 6.7). 

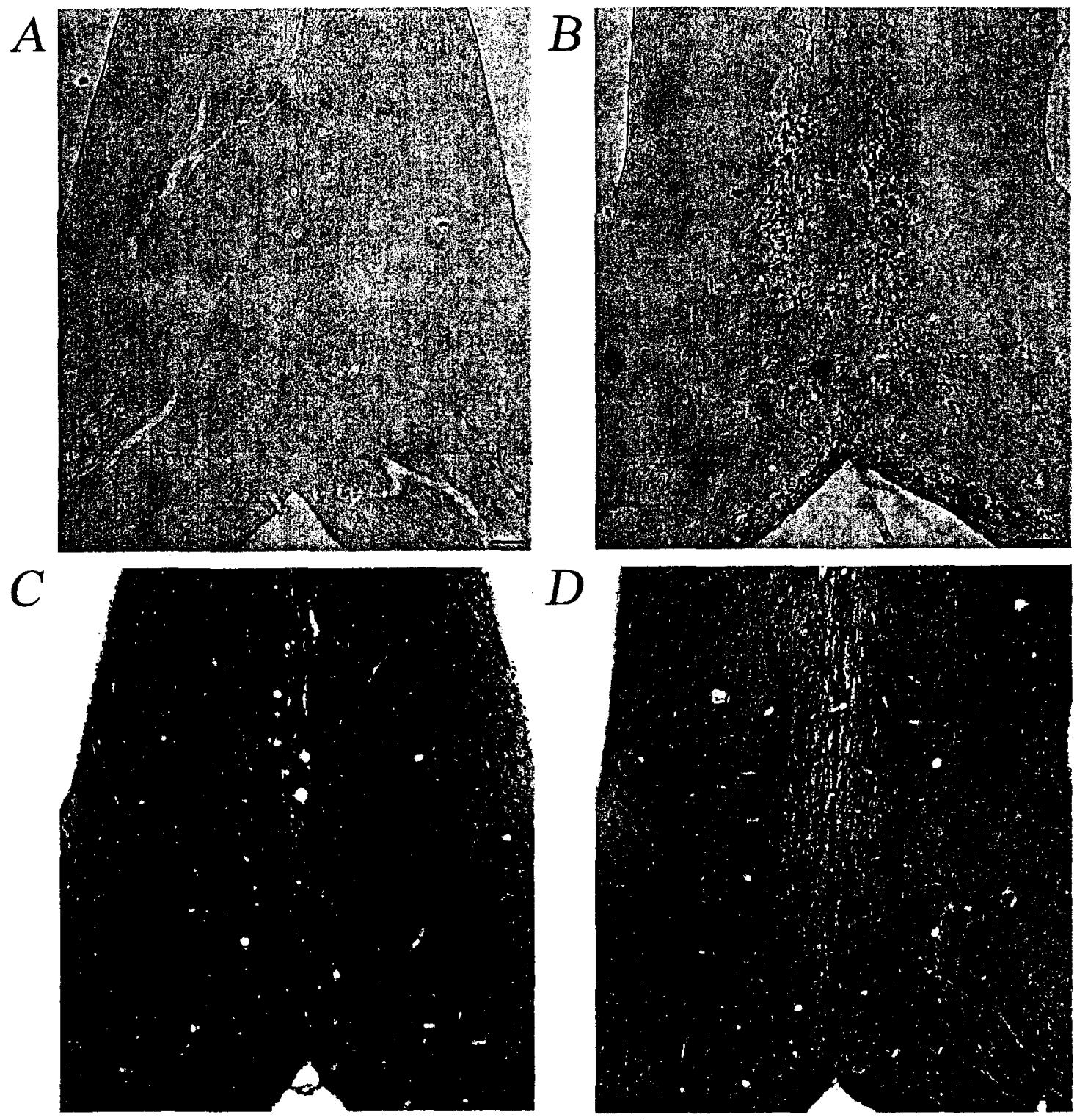

$D$

$E$
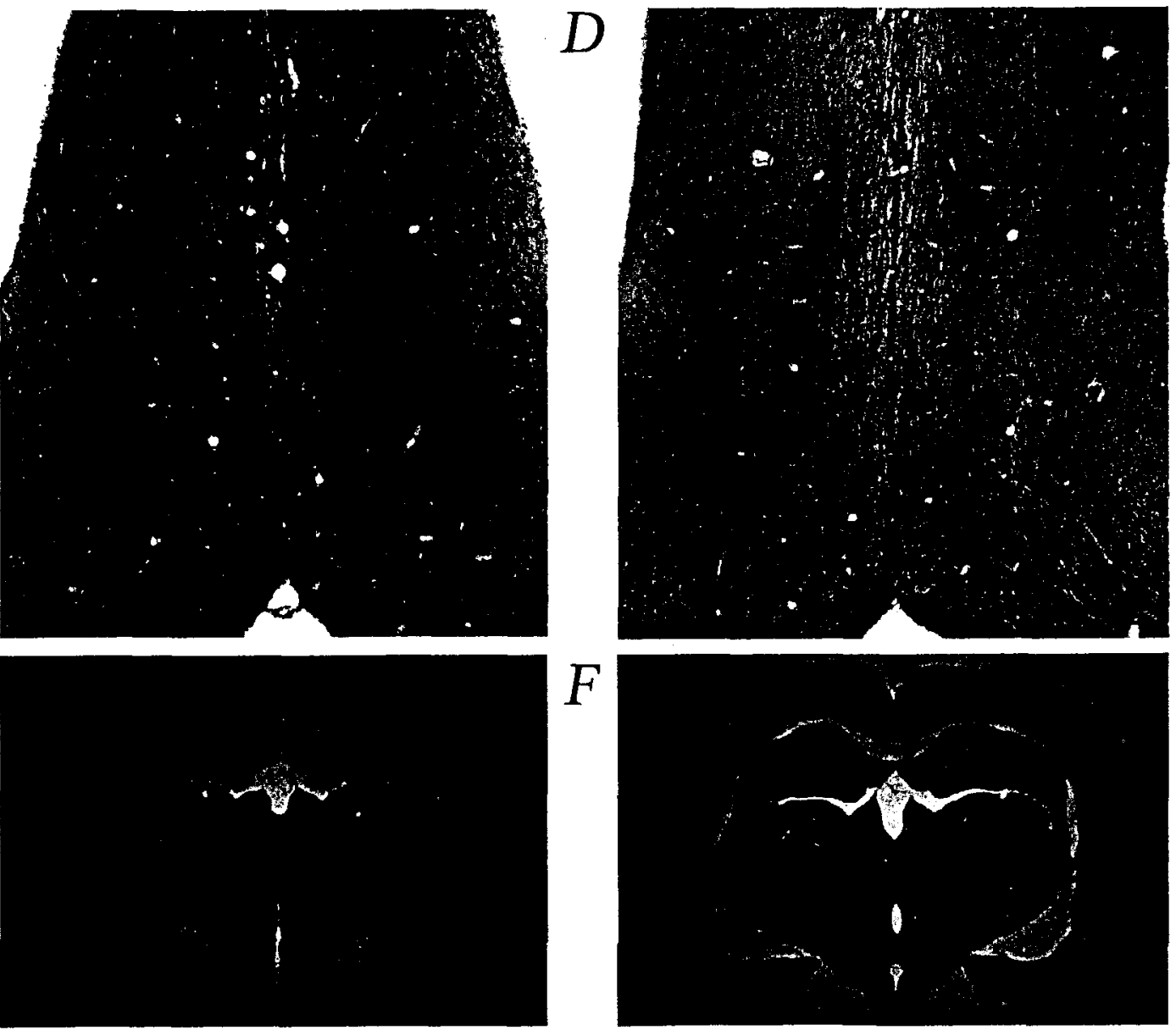

$F$

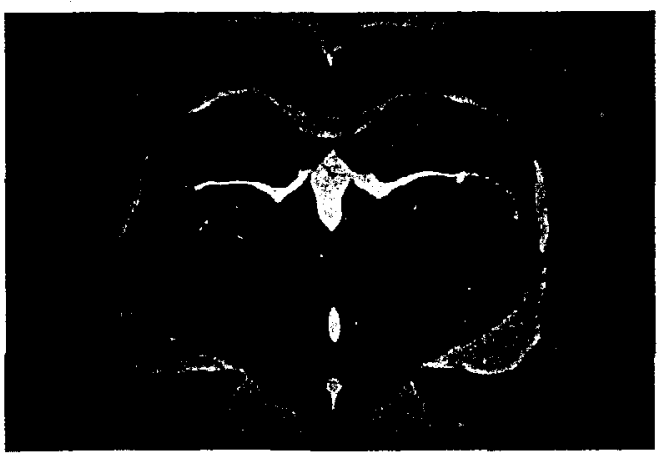

Figure 6.6: Extent of the cholinergic lesion. ChAT labeled cholinergic neurons (A,B), parvalbumin labeled GABA-ergic neurons $(\mathrm{C}, \mathrm{D})$ and $\mathrm{AChE}$ staining $(\mathrm{E}, \mathrm{F})$ in a representative lesioned $(A, C, E)$ and sham operated rat $(B, D, F)$. Scale bars, $0.1 \mathrm{~mm}(A-D)$ and $1.0 \mathrm{~mm}(\mathrm{E}-\mathrm{F})$. 
Table 6.1: The number of cholinergic neurons counted in two sections for rats averaged over all experiments.

\begin{tabular}{lccc}
\hline Group & \multicolumn{3}{l}{ Number of ChAT positive neurons } \\
\cline { 2 - 4 } & Section 1 & Section 2 & Total \\
\hline Control & $164.67+/-9.54$ & $161.33+/-21.91$ & $326.00+/-22.64$ \\
\hline AC & $6.12+/-2.40$ & $0.00+/-0.00$ & $6.12+/-2.40$ \\
\hline Stress & $134.33+/-12.57$ & $173.40+/-17.30$ & $307.73+/-22.34$ \\
\hline AC-stress & $5.43+/-2.14$ & $3.36+/-2.10$ & $8.79+/-3.96$
\end{tabular}

Data are given as means $+/$ - SEM. Control rats are from a separate experiment and included only as a general comparison.

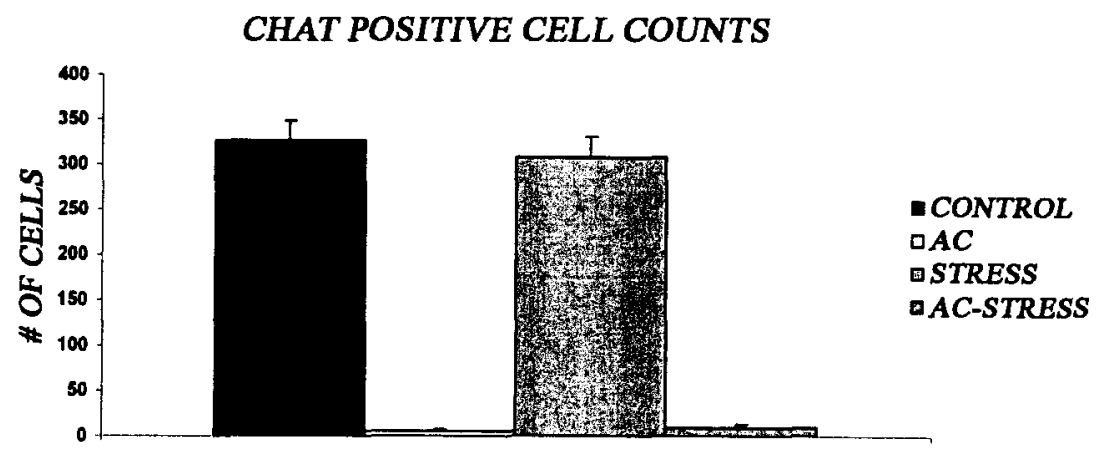

Figure 6.7: Choline acetyltransferase (ChAT) positive cell counts show a clear depletion in $\mathrm{AC}(98 \%)$ and $\mathrm{AC}$-stress $(97 \%)$ rats compare to Stress and Control rats. Control rats are from a separate experiment and included only as a general comparison. Data are given as means $+/$ - SEM.

Lesioned animals had areas of necrotic cell death in the MS/VDB surrounding the injection site. This non specific damage has been reported previously (Heckers et al., 1994; Frick et al., 2004) and work in our laboratory has shown no correlation between lesion size (due to focal necrosis following infusion of the toxin) and water maze or fear conditioning performance (data not shown). Analysis of Cresyl violet stained slides under a 10x objective did not reveal any changes in hippocampal structure (Figure 6.8). 


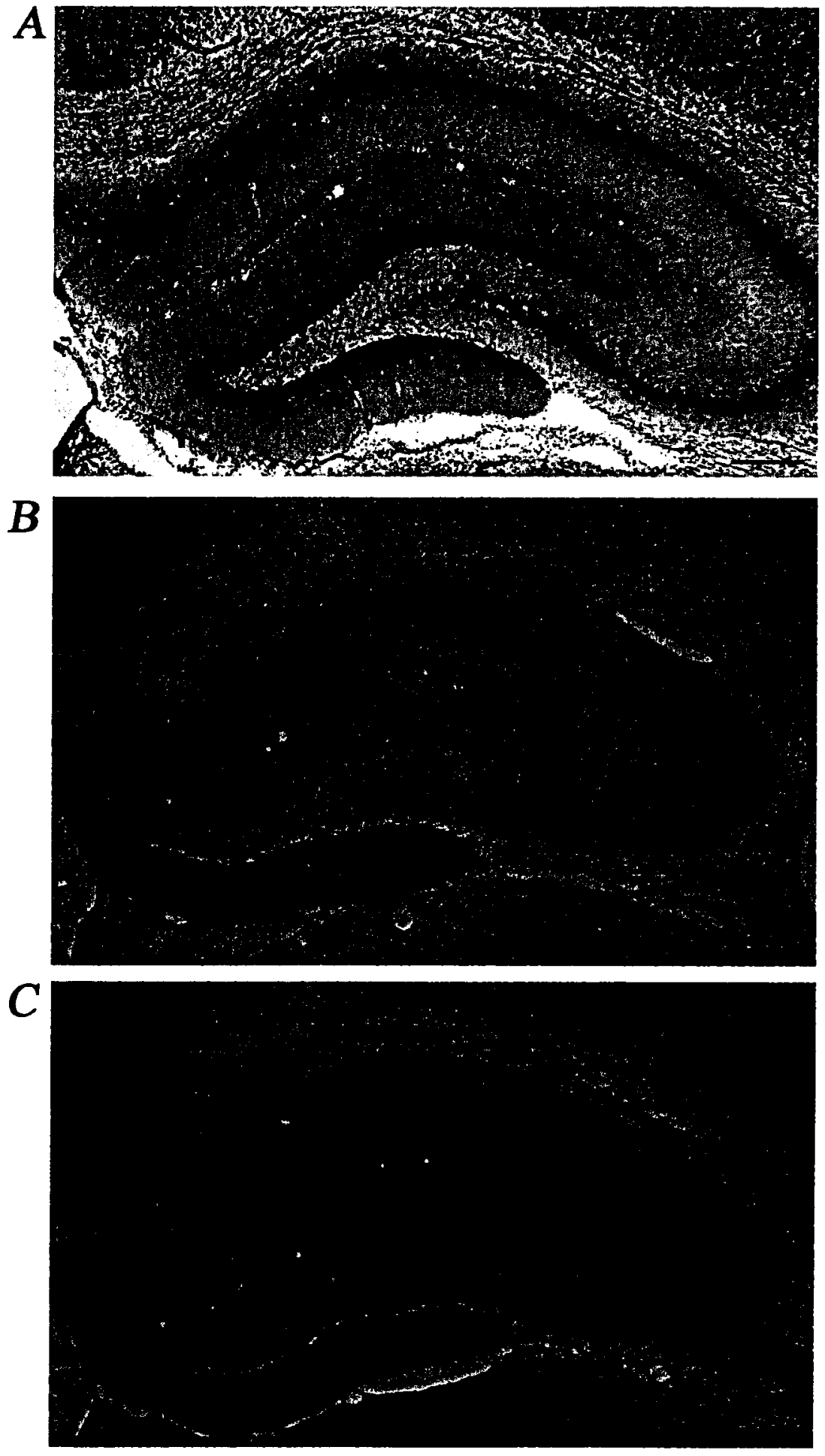

Figure 6.8: There was no gross hippocampal damage found in any of the experimental groups. Cresyl Violet stained sections from an $\mathrm{AC}$ rat (A), an AC-stress rat (B) and a Stress rat (C). Scale bars, $0.5 \mathrm{~mm}$. 


\section{Discussion}

In this study I show that the combination of two commonly occurring risk factors for $\mathrm{AD}$ (cholinergic depletion and chronic stress) specifically impairs performance in the water maze task, while having no effect on performance of fear conditioning to tone and context. The finding that these two factors could cause such a significant deficit was unexpected as both risk factors on their own have been previously found to have few or no effects on the behavioural tasks used here.

Cholinergic depletion of the medial septum has been extensively studied in our laboratory and others. To the best of my knowledge, there have been no reports of a spatial impairment in the water maze resulting from a specific cholinergic lesion of the medial septum (Baxter et al., 1995; Bizon et al., 2003; Frielingsdorf et al., 2006) and my finding here, of no impairment in my depleted rats, adds further support to the existing literature. A similar finding was evident in the fear conditioning task. While there have been no previous reports on fear conditioning following a specific depletion of the medial septum, Frick et al. (2004) completely damaged basal forebrain cholinergic neurons (including the medial septum) and found no difference in levels of freezing to tone or context. My results support their data and add to the growing body of literature describing the role of septo-hippocampal cholinergic projections in mediating cognitive processes.

Mild to moderate stress prior to learning is generally believed to have no effect on acquisition, but can impair retrieval and retention in certain situations (reviewed in Shors, 2006). The variable restraint stress used here, had no effect on spatial memory as demonstrated in the current study, and previously by our laboratory using the water maze 
task (McDonald et al., 2008). Other groups have reported similar results on spatial learning following mild stress (Luine et al., 1996; Gouirand et al., 2006, but see Radecki et al., 2005). Moreover, I did not observe any 'learned helplessness' behaviour in either of my stressed groups. This is in agreement with previous reports looking at the performance of rats in the water maze or forced swim test following restraint stress (Gregus et al., 2005; McDonald et al., 2008). The same pattern of results is found with fear conditioning. Here I have reported no effect on fear conditioning to tone and context following chronic stress. This supports the previous findings of Miracle et al. (2006), but Skorzewska et al. (2006) reported an enhancement following chronic CORT treatment. Taken together, these studies suggest that the type and length of stressor is critical to the appearance of a cognitive deficit. It is likely that although my variable stress procedure was able to increase CORT levels, it was not sufficient to alter performance on these tasks.

The impairment in the water maze, combined with successful performance on the fear conditioning task suggests that this was a hippocampal specific deficit. A similar pattern of results is observed following complete lesions of the hippocampus. Previous studies have shown that rats with anterograde damage to the hippocampus are consistently impaired on the acquisition of the spatial version of the water maze (Morris et al., 1982; Sutherland et al., 1983; Ferbinteanu and McDonald, 2003; McDonald et al., 2005). Furthermore, rats with anterograde damage to the hippocampus are not impaired at either fear conditioning to cue or context; however, rats with damage to the hippocampus after training impairs retention of fear conditioning to context but not to cue (Phillips and LeDoux, 1992; Sanders et al., 2003; Wiltgen et al., 2006). It would be interesting to see 
whether the combination of cholinergic depletion and stress administered after contextual fear conditioning would have a negative effect on retention. While this study has not been performed, I suspect that would be the case, providing further support to my claim that my manipulation specifically affects the hippocampus. It was surprising, given the extent of the observed impairment in spatial memory, that I did not detect any obvious damage or structural changes in the hippocampus. This suggests that a more subtle mechanism is interfering with hippocampal processing resulting in the observed deficit.

Possible mechanisms for the negative effect of stress in cholinergic depleted rats

Chronic stress and decreased levels of acetylcholine (ACh) are both commonly found in the aged population (McGeer et al., 1984; Fischer et al., 1991; Lupien et al., 1998; Baskerville et al., 2006; Montaron et al., 2006). Each of these factors has been shown to independently affect a number of mechanisms purported to lead to cognitive decline or memory deficits in the absence of gross hippocampal abnormalities. One way that decreased levels of ACh and chronic stress may interact to produce memory impairment is through their effects on neurogenesis. Hippocampal neurogenesis is believed to play a role in spatial learning and memory (Nilsson et al., 1999; Shors et al., 2001; Snyder et al., 2005). Both increased CORT and decreased levels of ACh, on their own, have been shown to significantly decrease hippocampal neurogenesis in the young rat (Cooper-Kuhn et al., 2004; Heine et al., 2004; Mohapel et al., 2005). The combination of the two factors would likely decrease levels even more which could alter hippocampal functioning, and potentially effect cognition and memory.

Changes in neuronal plasticity are a second possible way that chronic stress and cholinergic depletion could have negative effects on learning and memory. The 
cholinergic system has been implicated in mediating recovery from injury (Borlongan et al., 1995; Gonzalez et al., 2006) and in regulating the expression of neurotrophic factors such as brain derived neurotrophic factor (BDNF) and nerve growth factor (NGF) (Knipper et al., 1994; French et al., 1999). Chronic exposure to glucocorticoids can lead to hippocampal atrophy and neuronal cell death (Magarinos and McEwen, 1995; Sapolsky, 2000). A functional cholinergic system may play a role in compensating for the negative effects of chronic stress in the hippocampus.

\section{Support for the multiple combinations of co-factors theory of aging}

Previous work from our laboratory (Driscoll et al., 2007; McDonald et al, 2008) has suggested that the presence of a passive risk factor prior to the occurrence of an active risk factor results in increased hippocampal cell death leading to behavioural deficits. In the current study, I have proposed that the impairment results from the interaction of two passive risk factors, caused by an alternative mechanism, independent of gross hippocampal damage. This pattern of data suggests that different mechanisms underlie the cognitive deficits in the active/passive versus the passive/passive risk factor combinations. While the exact mechanism behind this vulnerability and resultant impairment is unknown, it is likely that reductions in neurogenesis, and subtle damage caused by prolonged exposure to CORT, in the absence of the neuroprotective effects of $\mathrm{ACh}$, has prematurely 'aged' these rats to a stage where cognitive impairments become apparent.

\section{Conclusion}

Here I have shown that the interaction of two passive factors (cholinergic depletion and stress) commonly found in the elderly population can lead to impairment in 
hippocampal dependent learning and memory. My findings suggest a role for the cholinergic system in providing protection for hippocampal neurons, or mediating compensatory mechanisms following injury. I do not see the decline in ACh levels in the elderly as a causal factor, but instead, as an important risk factor that leads to the increased susceptibility of an individual to develop AD. Fully understanding the contribution of multiple different risk factors for $\mathrm{AD}$, especially that of $\mathrm{ACh}$, and their interactions with one another could be vital to describing an accurate etiology of $\mathrm{AD}$. From that etiology, a series of effective treatment plans could be designed dependent on the specific risk factors present in each individual case. 


\section{Chapter 7}

Cholinergic depletion of the medial septum followed by phase shifting has no effect on circadian rhythms or memory in rats

Modified from a paper submitted to $\underline{\text { Brain Research Bulletin, }}$

by Laura A. Craig, Nancy S. Hong, Joelle Kopp and Robert J. McDonald in 2008 


\begin{abstract}
The robustness of an individual's circadian rhythms has been correlated with the quality of their cognitive aging. This has been observed in both human and non-human animals and circadian rhythms are especially disrupted in patients with Alzheimer's disease (AD). It is possible that the circadian disruption observed in $\mathrm{AD}$ contributes to the cognitive decline in these patients; however, this has not been conclusively proven. A common observation in $\mathrm{AD}$ patients is the loss of basal forebrain cholinergic neurons, some of which project to the suprachiasmatic nucleus (SCN) responsible for maintaining circadian rhythms. I was interested to see if cholinergic depletion increased susceptibility to circadian disruption, and to explore possible interactions between these two factors on measures of learning and memory. I damaged the cholinergic neurons of the medial septum in rats using the specific immunotoxin 192 IgG Saporin and then disrupted circadian rhythms using a six day phase shifting procedure. I looked at measures of circadian rhythmicity, as well as behaviour on tasks designed to test hippocampal dependent (water maze) or hippocampal independent (fear conditioning) learning and memory. I found no difference between the groups on any of the measures examined suggesting that the cholinergic depletion of the medial septum does not increase susceptibility to circadian disruption, and that this combination of risk factors does not contribute to learning and memory impairments.
\end{abstract}




\section{Introduction}

The suprachiasmatic nucleus $(\mathrm{SCN})$ in the brain is responsible for maintaining daily circadian rhythms (Antle and Mistleberger, 2005). The responsiveness of the SCN to light can be affected by cholinergic projections originating in the basal forebrain and brainstem nuclei (Bina et al., 1993, 1997). Several studies have provided evidence for a role of cholinergic signaling in the maintenance of circadian rhythmicity. Specifically SCN acetylcholine ( $\mathrm{ACh}$ ) has been shown to be up-regulated in response to light and infusions of cholinergic agonists can mimic the phase shifting effects of a light pulse (Zatz and Herkendam, 1982; Murakami et al., 1984; Ferguson et al., 1999). Moreover, cholinergic antagonists such as mecamylamine can block the phase shifting effects of a sub maximal light pulse (Keefe et al., 1987; Zhang et al., 1993). These studies suggest a role for ACh in the maintenance of healthy circadian rhythms.

Loss of cholinergic activity in the brain is common in $\mathrm{AD}$ patients. It is possible that the decrease in ACh reduces the ability of the SCN to respond appropriately to light leading to increased circadian disruption during aging. Circadian disruption is a common complaint in patient's suffering from $\mathrm{AD}$ and has been associated with cognitive impairments in both rats and humans (Fekete et al., 1985; Bonnet, 1989; Stone, 1989; Antoniadis et al., 2000). Antoniadis et al. (2000) have shown the strength of the circadian rhythm is correlated with the quality of cognitive aging in animals. Patients suffering from Alzheimer's disease consistently report a number of circadian disturbances including disrupted sleep, shortened REM and increase night time awakenings (Moe et al., 1995; Hatfield et al., 2004). It is possible that the circadian disruption observed in AD 
patients may actually be contributing to the severity of the dementia, and to the progressive worsening of the disorder.

In the current experiment, I was interested in the effects of damaging the cholinergic neurons of the medial septum on measures of circadian rhythmicity and the ability of the rat to re-entrain following a series of phase shifts. Previous studies damaging cholinergic basal forebrain neurons found decreases in neuropeptide synthesis in the SCN that could affect the strength of the circadian clock (Madeira et al., 2004). Damaging the clock could lead to increased reliance on external zeitgebers (time cues used to reset the clock such as light, food and social contacts). With this in mind I predicted that depleted rats would have more difficulty re-entraining to a novel light/dark (LD) cycle following phase shifting than controls.

I was also interested in exploring the effects of cholinergic depletion and phase shifting on measures of learning and memory. Previous work in our laboratory has suggested increased hippocampal vulnerability following medial septal cholinergic lesions in rats (Craig et al., 2008). Circadian disruption induced by phase shifting has been reported to affect a number of mechanisms that can be detrimental to hippocampal function, such as stress (Sakelleris et al., 1975; Cho, 2000; Cho et al., 2001), abnormal neuronal spiking (Buzsaki, 1984), changes in the secretion pattern of hormones such as melatonin and cortisol (Moore-Ede et al., 1984; Goichot et al., 1998) and sleep disturbances (Sei et al., 1992; Ohayon et al., 2002). Moreover, repeated phase shifts have been shown to impair spatial memory in rats, while a single phase shift had no effect (Craig and McDonald, 2008). I predicted that in the absence of a functional cholinergic 
system, rats would be less able to cope with the negative effects of a single session of phase shifting and exhibit cognitive impairment.

Here I looked at the effects of phase shifting in rats following cholinergic depletion of the medial septum. I predicted that these rats will be impaired in their ability to re-entrain following a single phase shifting session. I also expected that the combined group would demonstrate a specific impairment in the water maze task, while retaining the ability to successfully learn a fear conditioning to tone and context task. This would suggest that a hippocampal specific deficit had resulted from the converging effects of phase shifting and cholinergic depletion on hippocampal functioning.

\section{Experiment 1: The effects of cholinergic depletion of the medial septum on measures} of circadian rhythmicity and their response to phase shifting

My first experiment was designed to detect changes in the ability of the rat to entrain to a 12 hour LD schedule following cholinergic depletion of the medial septum. I then looked at the ability of these rats to re-entrain to a novel LD schedule after six days of circadian disruption.

\subsection{Materials and Methods}

\section{Animals}

Forty male Long Evans hooded rats (300-400g) were obtained from Charles River (Saint-Constant, $\mathrm{PQ}$ ) and used for both experiments. Rats were divided into four groups: AC ( $n=10)$ had a cholinergic depletion only; Phase shift $(n=11)$ had a sham surgery and then were phase shifted; AC-phase shift $(n=10)$ had a cholinergic depletion and then were phase shifted; and Control $(n=10)$ had a sham surgery only. Animals were housed individually in polypropylene cages equipped with a $42.5 \mathrm{~cm}$ diameter running 
wheel, with food and water ad libitum. Rats were originally entrained to a 12 hour LD schedule with lights off at 19:30. All animals were handled in accordance to guidelines set out by the Canadian Council for Animal Care.

\section{Surgery}

Surgery was performed under Isoflurane anaesthesia $(4 \%$ with $2.0 \mathrm{~L} / \mathrm{min}$ of oxygen for induction and $2 \%$ after surgical plane was established) in a standard stereotaxic apparatus. An incision was made in the scalp and periosteum down the midline. The fascia was pushed to the edges of the skull with a sterile gauze swab and the skin retracted to expose the skull surface. Holes were drilled into the skull using a 1 $\mathrm{mm}$ drill bit attached to a high speed drill. Following surgery all animals were given buprenorphine (Temgesic; $0.1 \mathrm{mg} / \mathrm{kg}$ subcutaneously) as an analgesic.

\section{Cholinergic depletion}

The specific neurotoxin 192 IgG Saporin (IgG SAP; Chemicon) has been commonly used to induce selective cholinergic lesions in rats depending on the site of infusion. I chose to damage cholinergic neurons in the medial septum and vertical limb of the diagonal band of Broca (MS/VDB) which leads to a reduction in levels of hippocampal acetylcholinesterase (AChE). I lowered a 30 gauge cannula, through holes drilled in the skull, into the MS/VDB (AP +0.45; ML +/-0.6; DV -6.6 and $-8.0 \mathrm{~mm}$ from bregma and the skull) and bilaterally infused IgG SAP $[0.15 \mu \mathrm{g} / \mu \mathrm{l}$ dissolved in sterile $1 \mathrm{x}$ phosphate buffer saline (PBS; pH 7.4)] at a rate of $0.05 \mu 1 / \mathrm{min}$ using a Harvard minipump. Each animal received a total volume of $0.3 \mu \mathrm{l}$ per side at the first injection site (DV -8.0), and $0.2 \mu \mathrm{l}$ per side at the second site (DV -6.6). The cannula was left in place for six and four minutes after the first and second injection respectively to ensure 
diffusion of the toxin. Sham operated rats underwent the identical procedure except they were infused with $1 \mathrm{x}$ sterile PBS instead of IgG SAP.

\section{Phase Shifting}

The phase shifting procedure used here was designed to create a state of circadian disruption for a specified length of time (six days) and to look at the ability of the rats to partially re-entrain prior to behavioural testing. This procedure has been previously used in our laboratory to examine learning and memory following circadian disruption (Devan et al., 2001; Craig and McDonald, 2008). Animals were phased advanced by three hours a day for six days. This phase advancement was followed by ten days of re-entrainment to allow the animals a partial recovery (ten days is approximately half the normal reentrainment time required in this paradigm; Devan et al., 2001). Table 7.1 shows the time of lights off for the duration of the phase shifting procedure.

Table 7.1: Schedule of phase shifting used to induce circadian disruption

\begin{tabular}{cc}
\hline DAY & \\
\hline 1 & Lights off at 16:30 \\
2 & Lights off at 13:30 \\
3 & Lights off at 10:30 \\
4 & Lights off at $07: 30$ \\
5 & Lights off at $04: 30$ \\
6 & Lights off at $01: 30$ \\
$7-16$ & Re-entrainment: Lights off at $22: 30$ \\
\hline
\end{tabular}




\section{Data Collection}

Animals were housed individually in polypropylene cages equipped with a 42.5 $\mathrm{cm}$ diameter running wheel attached via a microswitch to a computer. Animals were exposed to a 12 hour LD cycle and activity (wheel running) was monitored continuously using a ClockLab data analysis and collection system for circadian rhythms. Actograms for each animal were double plotted to clearly show general patterns of activity. Total activity per day, percentage of activity during the light phase, and period length were calculated for each animal using the ClockLab analysis program (ActiMetrics Software). All three measures were calculated for the four days before surgery (baseline), four days prior to the start of phase shifting session (pre) and for the four days following phase shifting (post). Activity was recorded in wheel revolutions per minute, and was separately calculated for the light phase as well as total activity per day. The period was calculated by fitting a regression line to activity onsets using ClockLab analysis software.

\subsection{Results}

Five rats assigned to the $\mathrm{AC}$ and $\mathrm{AC}$-phase shift groups were removed from this experiment due to incomplete cholinergic lesions ( $<40 \%$ cell loss). The following histological, behavioural and circadian rhythms analyses include six animals in the AC group and eight rats in the AC-phase shift group. Circadian rhythms were continuously recorded throughout the study using a running wheel connected to a computerized data collection system. Due to minor technical difficulties with the microswitches on the wheels, the data from one or two days was occasionally lost for individual rats. For these rats, the averages were calculated from the remaining days in each time segment. 


\section{Circadian rhythms}

A representative actogram is shown from each group illustrating the initial entrainment, phase shifting and re-entrainment for a Control rat (Figure 7.1A), a Phase shift rat (7.1B), an AC rat (7.1C), and an AC-phase shift rat (7.1D).
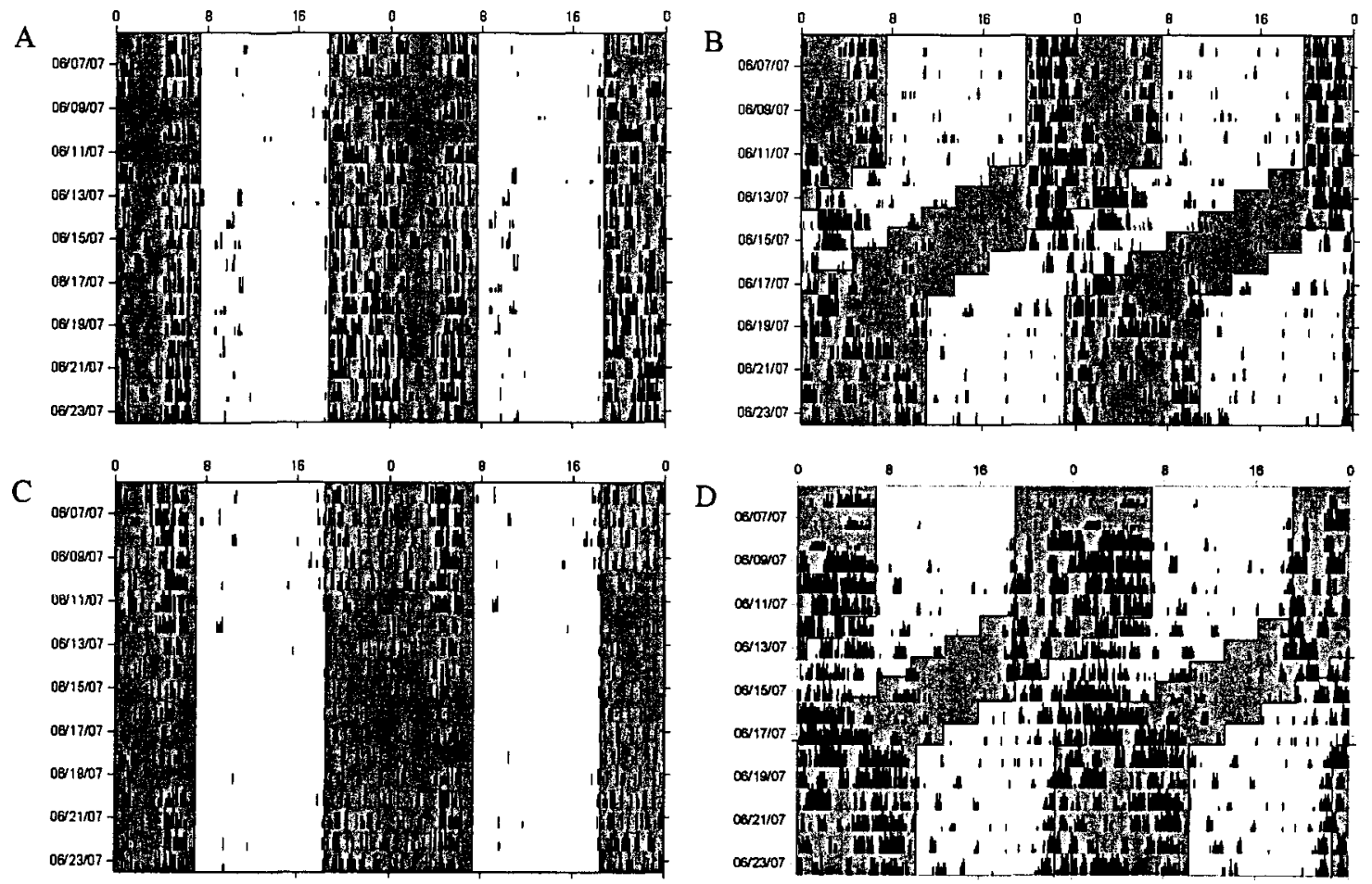

Figure 7.1: Representative actograms from a Control rat (A), a Phase shift rat (B), an AC rat (C) and an AC-phase shift rat (D) showing initial entrainment, phase shifting and reentrainment. Actograms are double plotted over forty eight hours for clarity. Each horizontal line represents two days, and the vertical bands represent wheel running. The shaded portions refer to when the lights were off.

During the initial entrainment the rats predictably began running shortly after lights off and ceased running when the time lights came on again. These onsets were used to determine the period length for each animal. The period was calculated for the four days before surgery (baseline), the four days before the phase shift (pre) and the four days 
immediately following the phase shift (post). All groups had a near 24 hour period which was not significantly affected by the phase shift (see Table 7.2 for a summary of circadian rhythm measures). A two-way mixed analysis of variance (ANOVA) with Group (Control versus AC versus Phase Shift versus AC-phase shift) as a between subjects variable, and Phase shift (baseline versus pre versus post) as the repeated measure found a marginally significant effect of group $[F(3,28)=2.95, p=0.05]$, but no effect of phase shift $[F(2,56)=0.61, p=0.55]$ and no interaction $[F(6,56)=2.01, p=0.08]$. Tukey post hocs found no difference between groups $(p$ 's $<0.05)$.

\section{Activity analysis}

Normal rats will confine the majority of their activity to the dark phase of the light cycle, while spending the light phase resting. Therefore, the proportion of activity during the light phase (light activity) is a good measure of rhythm fragmentation. During the initial entrainment the rats predictably confined their activity to the dark phase. Following the first phase shift, there was a slight increase in the proportion of light activity in both phase shifted groups, but this increase was not significant (Table 7.2). A two-way mixed ANOVA with Group as a between subjects variable and Phase Shift (three levels) as the repeated measure found no effect of group $[F(3,28)=0.39, p=0.76]$ or phase shift $[F(2,56)=0.82, p=0.45]$ and no interaction $[F(6,56)=1.63, p=0.16]$.

I also looked at total daily activity and found all groups decreased their activity over the course of this study. Total daily activity, measured as counts per day (cpd; one wheel revolution is equal to one count) was highest during initial entrainment (1645.54 +/- 351.12 cpd averaged over all groups) and gradually decreased after surgery (897.83 +/- $199.89 \mathrm{cpd})$, and again after the phase shift $(827.82+/-190.77 \mathrm{cpd})$. This drop in 
activity was most likely due to the effects of surgery (depletion or sham) and there were no differences between groups (Table 7.2). A two-way mixed ANOVA with Group as a between subjects variable, and Phase Shift (three levels) as the repeated measure found a significant effect of phase shift $[F(2,56)=3.61, p<0.05]$, but no effect of group $[F(3,28)=0.96, p=42]$ and no interaction $[F(6,56)=0.30, p=94]$. Tukey post hocs revealed that all groups became slightly less active following surgery $(p=0.06)$ and following the phase shift were significantly less active $(p$ 's $<0.05)$.

There were no effects of cholinergic depletion of the medial septum on the ability of rats to entrain to a 12 hour LD schedule. Following phase shifting, there were no differences between groups on measures of period length, total activity and rhythm fragmentation.

Table 7.2: Measures of circadian rhythmicity

\begin{tabular}{cccccc}
\hline & & Control & AC & Phase shift & AC-phase shift \\
\hline Total & Baseline & $921.61+/-248.27$ & $1514.65+/-465.17$ & $1843.92+/-701.30$ & $2268.97+/-1026.24$ \\
Activity & Pre & $603.95+/-154.38$ & $513.96+/-130.70$ & $1173.93+/-347.04$ & $1173.44+/-712.75$ \\
& Post & $632.17+/-189.30$ & $483.83+/-242.85$ & $1062.36+/-339.10$ & $964.86+/-627.50$ \\
\hline Light & Baseline & $13.80+/-3.86$ & $13.96+/-3.92$ & $10.33+/-1.63$ & $9.47+/-2.57$ \\
Activity & Pre & $10.50+/-2.19$ & $13.58+/-2.62$ & $12.03+/-2.48$ & $14.30+/-3.54$ \\
& Post & $9.04+/-1.73$ & $10.85+/-4.23$ & $19.77+/-3.73$ & $17.31+/-4.27$ \\
\hline Period & Baseline & $24.04+/-0.09$ & $23.94+/-0.07$ & $23.99+/-0.11$ & $23.96+/-0.07$ \\
& Pre & $24.07+/-0.09$ & $24.16+/-0.05$ & $24.11+/-0.08$ & $24.00+/-0.07$ \\
& Post & $23.86+/-0.07$ & $23.84+/-0.08$ & $24.50+/-0.15$ & $24.00+/-0.30$ \\
\hline
\end{tabular}

Data are given as means $+/$ - SEM. Total activity is measured in counts per day, Light activity is measured as \% of total activity and Period is measured in hours. 
Experiment 2: The effects of cholinergic depletion of the medial septum followed by phase shifting on measures of learning and memory

This experiment was designed to explore the effects of cholinergic depletion of the medial septum and phase shifting on measures of learning and memory. Rats were tested on a version of the spatial water maze task as a test of hippocampal dependent memory and on fear conditioning to tone and context to examine hippocampal independent memory.

\subsection{Materials and Methods:}

\section{Animals}

The same animals used in Experiment 1 were used for behavioural testing.

\section{Behavioural Testing}

Behavioural testing began ten days following the phase shifting session. To control for possible effects of circadian phase on performance, all groups were tested at the equivalent circadian phase. Phase shifted rats were tested at 13:30 (three hours after lights on), while non-phase shifted rats were tested at 10:30 (three hours after lights on). Behavioural testing consisted of a six day version of the water maze task to test for deficits in hippocampal processing, followed by three days on a cued and contextual fear conditioning task.

\section{Water maze: Rapid Acquisition Task}

I used a three stage spatial version of the water maze task (McDonald et al. 2005). A white plastic circular pool $1.4 \mathrm{~m}$ in diameter and $40 \mathrm{~cm}$ deep was filled to within $20 \mathrm{~cm}$ of the top of the wall with water $\left(20-22^{\circ} \mathrm{C}\right)$ made opaque by adding skim milk powder. 
The invisible platform was $12 \mathrm{~cm}$ in diameter and made of white Plexiglas with holes drilled into the top of it to provide grip for the animals. During training the platform was submerged $2 \mathrm{~cm}$ under water. Extra-maze cues in the training room included five posters of different sizes and orientations mounted on three of the four walls, a computer rack, a door, an animal rack and the experimenter. Four different start points were used [N, S, E, W (not true compass headings)], equally spaced around the pool. The order was randomly selected for each day and each start point was used twice for days one to four and day six. On day five only the three start points farthest from the platform were used $(\mathrm{N}, \mathrm{E}, \mathrm{S})$ with the initial start point repeated at the end for a total of 16 trials.

In the first stage (Original location training) rats were trained over four days for eight trials a day, to swim to a fixed, spatial location. Twenty four hours after completion of stage one the second stage (New location training) began. This stage consisted of new spatial location training as animals were re-trained to swim to the new platform location over sixteen trials within two hours on a single day. Stage two allowed me to ask if rats could learn to go to a new location, during a single intensive training session. The third stage (Re-training to original location) occurred twenty four hours after stage two with the platform returned to its original (stage one) location and each animal was given eight trials. This stage allowed me to ask how the massed new location training during stage two affected re-learning to the original location.

This version of the water maze was selected due to its sensitivity to hippocampal damage. The first stage allowed me to identify impairment in acquisition and short term memory of an invariant location. If no differences were apparent, the second stage would test the ability of the rat to rapidly form a novel representation in a familiar context. For 
the third stage the rats are required to re-learn the original location. There is evidence that these stages place a higher-demand on hippocampal function so they may allow me to detect more subtle hippocampal dysfunction (McDonald et al., 2005).

Data collection: A computer based rat tracker (Ethovision) was used to collect and analyze data obtained from an overhead video camera. If a rat did not reach the platform 60 seconds after release, the rat was guided manually to the platform and allowed to remain on the platform for 10 seconds before being removed to a holding cage until the next trial. For all three stages the measure of performance was latency to platform (in seconds) as well as path length to platform (in meters). Path length is a highly sensitive measure that can control for potential differences in swim speed between groups.

\section{Fear conditioning}

Twenty four hours after the final day of water maze training, rats were tested on a fear conditioning task. A three day procedure was used to assess fear conditioning to tone and to context. Animals were placed into Context A (an operant chamber, metal walls, bars on floor, scented with Quatsyl $@-D$ Plus disinfectant) and allowed to habituate for two minutes. They were presented with a 10 second tone $(80 \mathrm{db})$, followed by a two second shock (1.0 mA). This tone-shock pairing was repeated every 70 seconds for five repetitions. Sixty seconds following the final shock, the animals were removed from the chamber, and returned to their home cage. Twenty four hours later the animals were placed in a novel context, Context B (Plexiglas walls, bedding on the floor, unscented), and after two minutes of habituation, were presented with an eight minute tone $(80 \mathrm{db})$, with no shock and scored for freezing behaviour. Freezing behaviour is commonly used as a reliable measure of fear, and can be defined as a period of complete immobility 
except for breathing movements in the animal (Blanchard and Blanchard, 1969). Forty eight hours later animals were returned to Context $\mathrm{A}$ for a five minute trial (no shock, no tone) to assess freezing behaviour in response to the original conditioning context.

\subsection{Results}

\section{Water maze: Rapid Acquisition Task}

\section{Original location training}

The results of the original platform location training are depicted in Figure 7.2A. There were no differences between groups as all rats were able to learn the platform location during the original location training stage of this task. All groups showed a significant decrease in latency to find the platform from day one $(26.67+/-3.25$ seconds $)$ to day four $(7.67+/-0.87$ seconds). The latency (in seconds) to find the platform was recorded and averaged over the eight daily trials to obtain a single daily latency score for each animal. The daily scores for all four days of original location training were subjected to a two-way mixed ANOVA with Group (AC-phase shift versus AC versus Phase shift versus Control) as the between subjects variable, and Training Day (four levels) as a repeated measure, and revealed a significant effect of day $[F(3,93)=90.91$, $p<0.01]$, but no effect of group $[F(3,31)=2.14, p=0.12]$ or interaction $[F(9,93)=0.64$, $p=0.76]$.

The same pattern of results was found using path length to platform as the dependent variable (7.2B). This is a highly reliable measure that can control for potential differences in swim speed. A two-way mixed ANOVA with Group as a between subjects variable and Training Day as a repeated measure, revealed a significant effect of day 
$[F(3,93)=100.26, p<0.01]$, but no effect of group $[F(3,31)=2.02, p=0.13]$ or interaction $[F(9,93)=0.51, p=0.86]$.

\section{New location training}

The platform was moved to the opposite quadrant of the pool and the latency (in seconds) to find the new platform location was recorded and averaged into eight discrete trial blocks (two trials per block). There were no differences between groups in their ability to rapidly acquire a new platform location on this stage of the task (Figure 7.2C). All groups showed a significant decrease in latency to find the platform from trial block one $(34.90+/-5.09$ seconds) to trial block eight $(8.72+/-2.57$ seconds). A two-way mixed ANOVA with Group as a between subject variable and Trial Block (eight levels) as a repeated measure, was used to assess the new location training and found a significant effect of trial block $[F(7,217)=42.15, p<0.01]$, but no effect of group $[F(3,31)=1.34, p=0.28]$ or interaction $[F(21,217)=1.34, p=0.15]$.

The same pattern of results was observed using path length to platform as the dependent measure (7.2D). A two-way mixed ANOVA with Group as the between subjects variable and Trial Block as the repeated measure was performed and found a significant effect of trial block $[F(7,210)=43.74, p<0.01]$, but no effect of group $[F(3,30)=1.38, p=0.27]$ or interaction $[F(21,210)=1.02, p=0.44]$. 

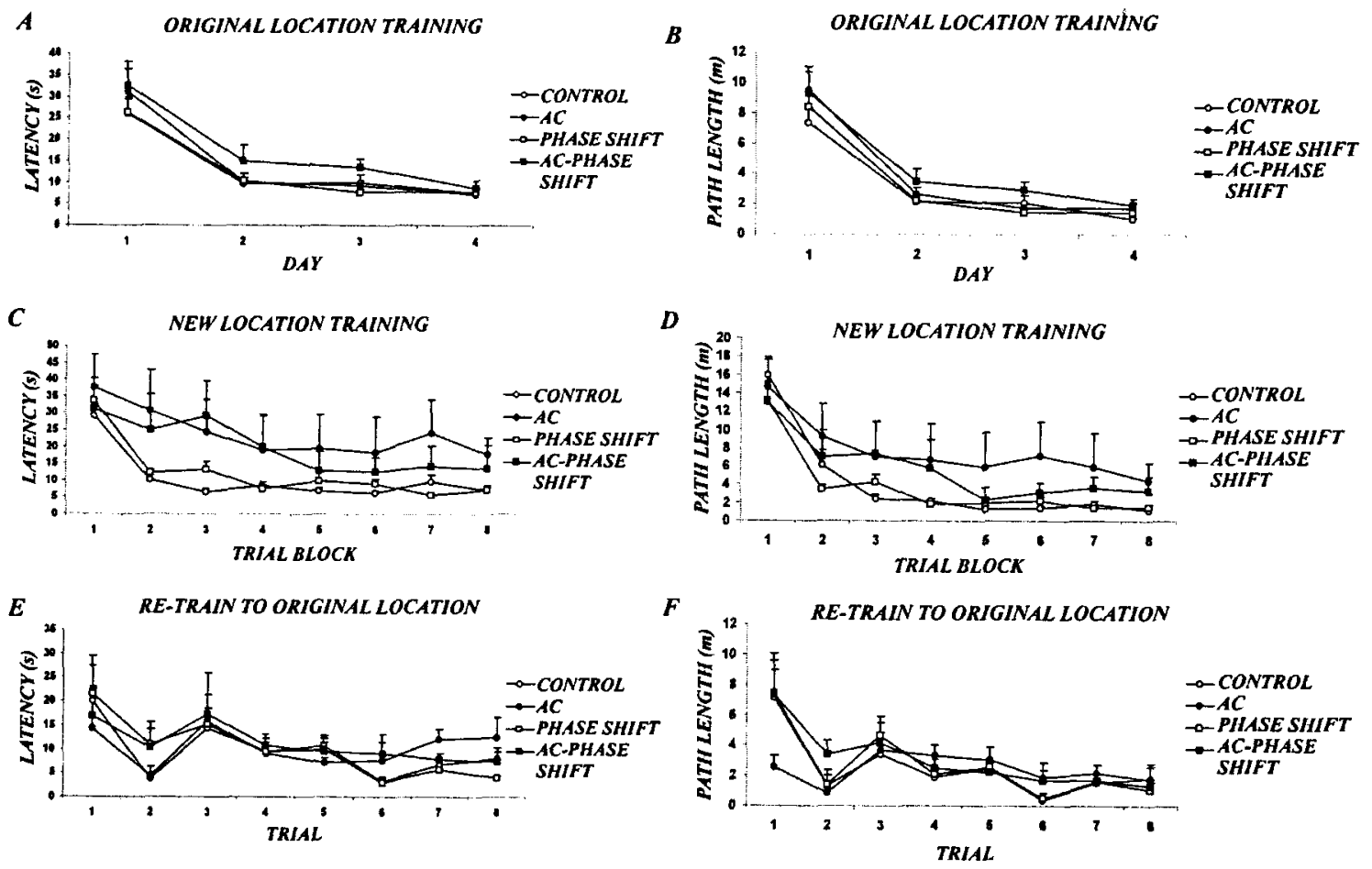

Figure 7.2: All groups were able to successfully learn all stages of the spatial water maze task. Both latency to platform and path length data is shown for all groups on original location training $(A, B)$, new location training $(C, D)$ and re-training to the original platform location (D,E). Data are given as means +/- SEM.

\section{Re-training to original location}

The platform was returned to the original location (from the acquisition phase) and the latency (in seconds) to find this location was recorded over eight trials on the final day of testing. The results of the re-training trials are displayed in Figure 7.2E showing that all groups were able to re-learn the original location. All groups showed a significant decrease in latency to find the platform from trial one $(19.95+/-5.12$ seconds $)$ to trial eight $(6.72+/-2.01$ seconds). A two-way mixed ANOVA, with Group as the between subjects variable, and Trial (eight levels) as a repeated measure revealed a significant effect of trial $[F(7,217)=42.15, p<0.01]$, but no effect of group $[F(3,31)=1.34$, $p=0.28]$ or interaction $[F(21,217)=1.34, p=0.15]$. 
The same pattern of results was seen using path length to platformas the dependent measure (7.2F). A two-way mixed ANOVA with Group as the between subjects measure and Trial as the repeated measure revealed a significant effect of trial $[F(7,196)=9.34, p<0.01]$, but no effect of group $[F(3,28)=0.28, p=0.84]$ or interaction $[F(21,196)=1.07, p=0.38]$.

There was no effect of combining cholinergic depletion and phase shifting on performance of rats on a hippocampal dependent task (water maze). All groups were able to successfully learn the platform location on all three stages of the water maze task.

\section{Fear conditioning}

The three day fear conditioning procedure used here was designed to test hippocampal independent learning and memory on two related tasks: fear conditioning to tone (cue) and to context. A malfunction with the shock boxes resulted in ten rats being removed from the analysis, leaving the final groups as follows: AC- $n=3$; AC-phase shift$n=6$; Phase shift- $n=9$ and Control- $n=7$.

\section{Conditioning to tone}

All groups were able to learn the tone-shock association, and showed an increase in freezing behaviour in the presence of the tone. The percentage of freezing during the first four minutes exposed to the tone was calculated and compared to the Context B habituation period. This percentage of freezing for all groups was significantly greater in the presence of the tone $(72.28 \%+/-9.54 \%$ versus $18.12 \%+/-4.47 \%$; Figure $7.3 \mathrm{~A})$. A two-way mixed ANOVA with Group as a between subjects variable, and Tone (habituation versus tone presentation) as a within subjects variable, revealed a significant effect of tone $[F(1,21)=77.72, p<0.001]$, but no group effect $[F(3,21)=2.22, p=0.12]$ or 
interaction $[F(3,21)=0.83, p=0.49]$. Tukey post hocs showed that all groups froze significantly more in the presence of the tone $\left(p^{\prime} s<0.05\right)$.

Conditioning to context

All groups were able to learn the context-shock association, and showed an increase in freezing behaviour when returned to Context A on day three. I measured the percentage freezing for the total time exposed to Context A on day three and compared it to the Context A habituation on day one. The percentage of freezing to context for all groups was significantly greater on day three compared to day one, although AC-phase shift and Phase shift rats showed a larger increase in freezing behaviour compared to $\mathrm{AC}$ and Control rats $(69 \%$ and $78 \%$ increase in AC-phase shift and Phase shift rats versus $45 \%$ and $37 \%$ increase in $\mathrm{AC}$ and Control rats; Figure 7.3B). A two-way mixed ANOVA with Group as a between subjects variable, and Context (day one versus day three) as a within subjects variable, revealed a significant effect of context $[F(1,21)=265.28$, $p<0.01]$, and of group $[F(3,21)=3.15, p=0.046]$, but no interaction $[F(3,21)=2.17$, $p=0.12]$. Tukey post hocs showed that all groups froze significantly more to the context on day three $(p$ 's $<0.05)$. Further analysis of the group effect using Tukey post hocs found no difference between groups, however Holm-Bonferroni post hocs revealed significantly more freezing in AC-phase shift and Phase shift rats when compared to Control rats $\left(p^{\prime} \mathbf{s}<0.05\right)$ 
A

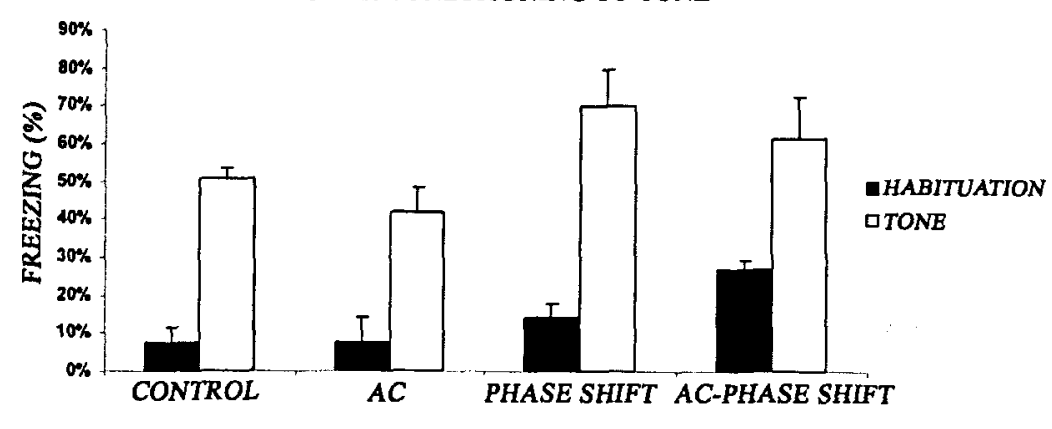

B

FEAR CONDITIONING TO CONTEXT

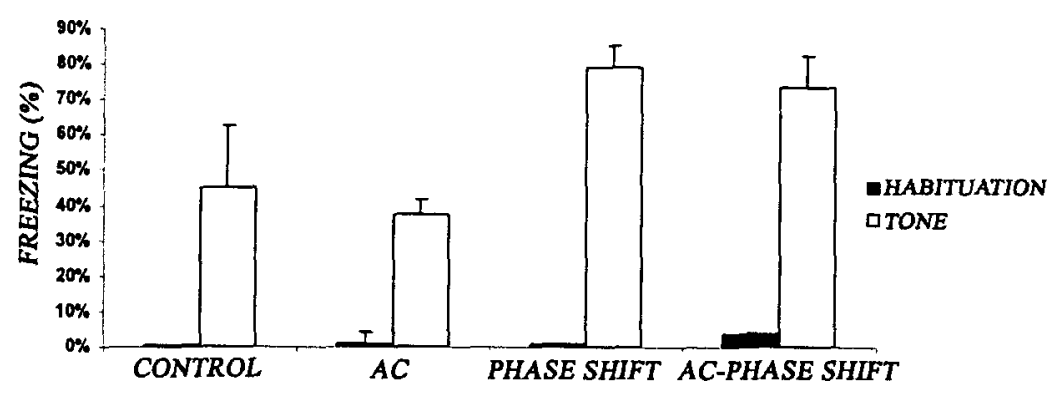

Figure 7.3: Performance on the fear conditioning task. There was no impairment in any of the groups on either fear conditioning to tone (A) or to context (B). Both phase shifted groups exhibited significantly more freezing in response to tone, but all groups demonstrated successful conditioning to tone in this task. Data are given as means $+/$ SEM.

Following the combination of cholinergic depletion and phase shifting, rats retained their ability to successfully learn a hippocampal independent task (fear conditioning to tone and context). Moreover, the phase shifting appears to enhance freezing behaviour as both AC-phase shift and Phase shift rats tended to freeze more than AC or Control rats.

\section{Histology}

Following behavioural testing all animals were sacrificed with an overdose of Euthanol and transcardially perfused with 1x PBS followed by $4 \%$ (w/v) paraformaldehyde (PFA). The brains were carefully extracted and placed in 4\% PFA at 
$4^{\circ} \mathrm{C}$ for 24 hours. Brains were then transferred to a $30 \%(w / v)$ sucrose solution, containing $0.02 \%$ (w/v) sodium azide at $4^{\circ} \mathrm{C}$ for at least three days. Brains were sectioned on a freezing cryostat at $40 \mu \mathrm{m}$ in a series of five. The first two sections of each series were mounted onto $1 \%(\mathrm{w} / \mathrm{v})$ gelatin $/ 0.2 \%(\mathrm{w} / \mathrm{v})$ chromalum coated slides, while the third, fourth, and fifth series were stored in $1 \mathrm{x}$ PBS containing $0.02 \%(\mathrm{w} / \mathrm{v})$ sodium azide at $4^{\circ} \mathrm{C}$ until being processed for immunohistochemistry. The fifth series was not used in this experiment and was stored for future analyses.

\section{Cresyl violet staining}

The first series was stained with Cresyl violet and examined under a 10x objective to detect any gross hippocampal damage.

\section{AChE staining}

The second series was stained for AChE to get a qualitative image of cholinergic depletion in the hippocampus. I treated the slides for 30 minutes with $6.24 \mathrm{mg}$ tetraisopropylpyrophosphoramide (Sigma) dissolved in $200 \mathrm{~mL}$ of distilled water, then rinsed the slides twice with distilled water and incubated them on a shaker table for four hours in a reaction mixture containing $5.72 \mathrm{~g}$ Trizma maleate, $2.04 \mathrm{~g}$ Trizma base, 100 mg acetylthiocholine iodide, $294 \mathrm{mg}$ dihydrous trisodium citrate, $150 \mathrm{mg}$ anhydrous cupric sulphate, $32.8 \mathrm{mg}$ potassium ferricyanide dissolved in $200 \mathrm{~mL}$ distilled water (all chemicals were obtained from Sigma). Slides were rinsed twice with distilled water, and left to dry overnight, then cleared with HemoDe (Sigma) and coverslipped with Permount (Sigma) the next day. 


\section{Immunohistochemistry}

The third and fourth series were processed for immunohistochemistry to determine the completeness and specificity of the cholinergic depletion. An antibody raised against parvalbumin was used to label $\gamma$-aminobutyric acid (GABA)-ergic neurons, and one against choline acetyltransferase (ChAT) was used to label cholinergic neurons. Free floating sections were rinsed in 1x PBS, and then placed in a $3 \%(v / v) \mathrm{H}_{2} \mathrm{O}_{2}$ solution for four minutes to block endogenous peroxidases. After three washes in 1x PBS, sections were blocked for 30 minutes in $15 \%(\mathrm{v} / \mathrm{v})$ normal horse serum (for antiparvalbumin) or $15 \%(\mathrm{v} / \mathrm{v})$ normal rabbit serum (for anti-ChAT). Sections were incubated overnight in primary antibody [anti-ChAT polyclonal, 1:1000 (Chemicon) or antiparvalbumin monoclonal, 1:5000 (Sigma)] diluted in 1x PBS with 7.5\% (v/v) serum (horse for anti-parvalbumin; rabbit for anti-ChAT). Sections were then washed three times in 1x PBS and incubated for 30 minutes in a biotinylated secondary antibody [ABC Elite Kit (mouse for parvalbumin and goat for ChAT), Vector Labs], washed three times, incubated for 30 minutes with $\mathrm{AB}$ reagent ( $\mathrm{ABC}$ Elite Kit, Vector Labs), washed three times then developed with diaminobenzidine (DAB) substrate (Vector Labs) according to the manufacturer's instructions. Sections were then mounted onto $1 \%(\mathrm{w} / \mathrm{v})$ gelatin $/ 0.2 \%$ (w/v) chromalum coated slides, air dried overnight, dehydrated in alcohol, cleared in HemoDe and coverslipped with Permount (Sigma).

Cell counts: Under light microscopy (AxioVision) ChAT positive neurons were counted in both hemispheres of the MS/VDB. I chose two sections to quantify; the first, at the site of injection and the second, in a section $200 \mu \mathrm{m}$ caudal to the injection site. This quantification was not used as an accurate count of MS/VDB neurons, but instead to 
estimate the completeness of the cholinergic depletion. Each hemisphere was counted separately to confirm lesion symmetry, after which counts for both hemispheres were totaled for a representative cell count over the two sections. I combined the ChAT positive cell counts from the Phase shift and Control groups, and used this average to determine the percent depletion for the AC and AC-phase shift groups. Any animal with less than $40 \%$ depletion was excluded from the experiment.

\section{Histological Results}

Quantification of cholinergic lesion

I confirmed the completeness and specificity of the cholinergic lesion of the medial septum in both the $\mathrm{AC}$ and $\mathrm{AC}-$ phase shift groups through $\mathrm{AChE}$ staining and immunohistochemistry for ChAT and parvalbumin. Lesioned animals had very few ChAT positive cholinergic neurons (Figure 7.4A-C) combined with intact parvalbumin labeled GABA-ergic neurons outside the area of necrosis, confirming the specificity of the lesion to cholinergic neurons (7.4D-F). The AChE staining showed a depletion of cholinergic activity in the hippocampus in lesioned animals compared to the sham operated groups $(6 \mathrm{G}-\mathrm{I})$. 

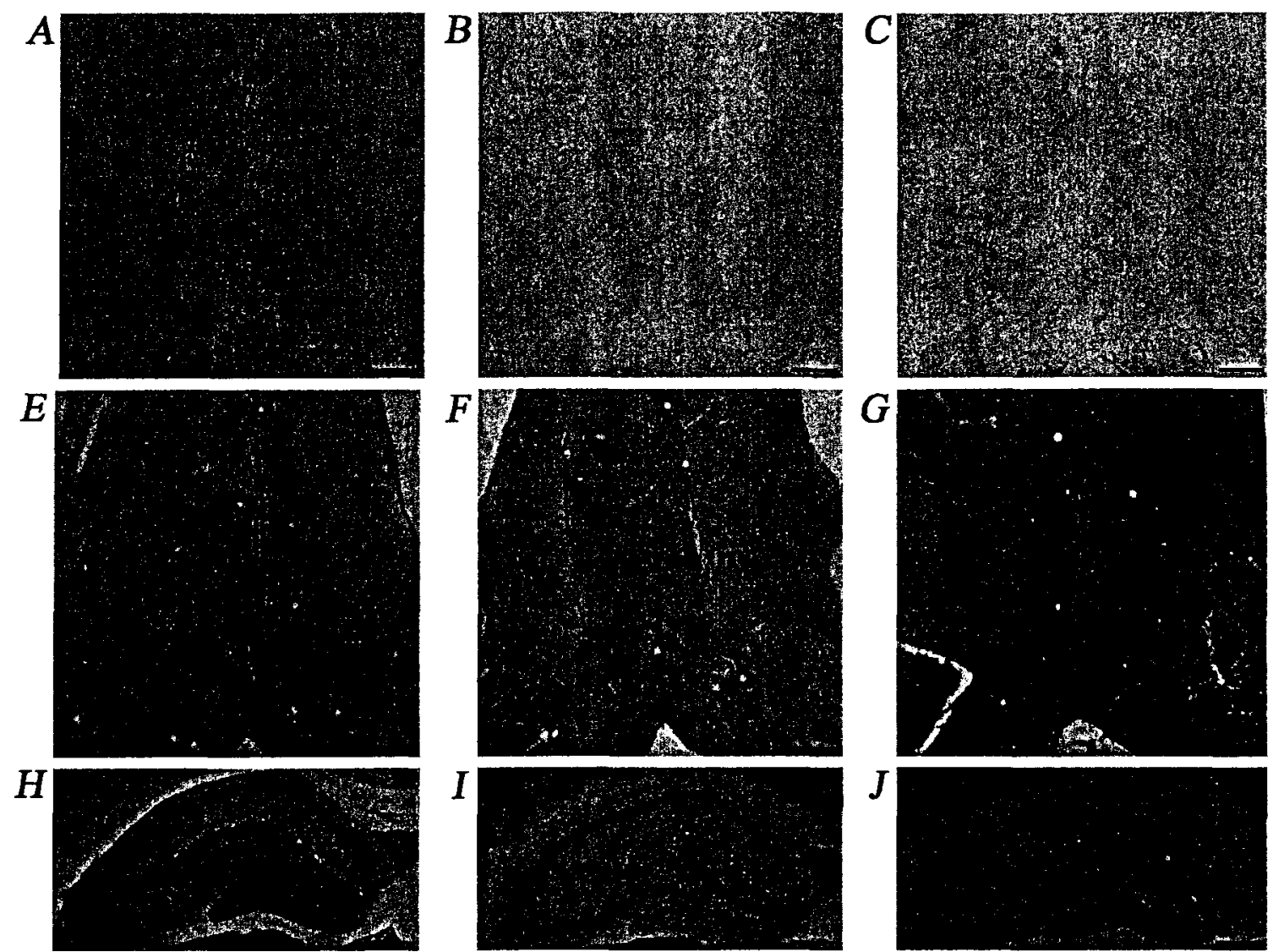

Figure 7.4: Extent of the cholinergic lesion. ChAT labeling in the MS/vDB of a Control rat (A), an AC rat (B) and an AC-phase shift rat (C) confirm the near total loss of cholinergic neurons in this area following infusion of IgG SAP. Parvalbumin labeled GABA-ergic neurons in the MS/vDB from a Control rat (D), an $\mathrm{AC}$ rat (E) and an $\mathrm{AC}$ phase shift rat $(F)$ confirm the specificity of this lesion to cholinergic neurons. Normal AChE staining apparent in the dorsal hippocampus (1x) of a Control rat $(G)$ is reduced following cholinergic depletion in the hippocampus of an $\mathrm{AC}$ rat $(\mathrm{H})$ and an $\mathrm{AC}$-phase shift rat (I). Scale bars, $0.1 \mathrm{~mm}(A-F)$ and $1.0 \mathrm{~mm}(\mathrm{G}-\mathrm{I})$.

I quantified the extent of the cholinergic lesion by comparing the ChAT positive cell counts between groups. I combined the ChAT positive cell counts from the Phase shift and Control groups, and used this average to determine the percent depletion for the lesioned groups. I found $70.91+/-3.73 \%$ depletion in the $\mathrm{AC}$ group and $71.04+/-8.00 \%$ depletion in the AC-phase shift group. An independent samples t-test showed no significant difference between the amount of depletion in the two groups $[t(12)=-0.01$, 
$p=0.99]$. There were significantly fewer cholinergic neurons in AC and AC-phase shift compared to the Phase shift and Control group [Table 7.3; $F(3,31)=13.52, p<0.01$ ]. Tukey post hocs showed that the AC and AC-phase shift groups had significantly fewer ChAT positive cells than either of the Phase shift and Control groups ( $p$ 's $<0.05$; Figure $7.5)$.

Table 7.3: The number of cholinergic neurons counted in two sections.

\begin{tabular}{llcl}
\hline Group & \multicolumn{3}{l}{ Number of ChAT positive neurons } \\
\cline { 2 - 4 } & Section 1 & Section 2 & Total \\
\hline Control & $93.90+/-8.67$ & $106.30+/-12.34$ & $200.20+/-17.61$ \\
AC & $26.00+/-6.49$ & $33.50+/-6.47$ & $59.50+/-7.62$ \\
Phase shift & $89.91+/-16.75$ & $118.64+/-15.51$ & $208.55+/-28.81$ \\
AC-phase shift & $21.13+/-6.70$ & $38.13+/-10.43$ & $59.25+/-16.34$ \\
\hline
\end{tabular}

Data are given as means +/- SEM.

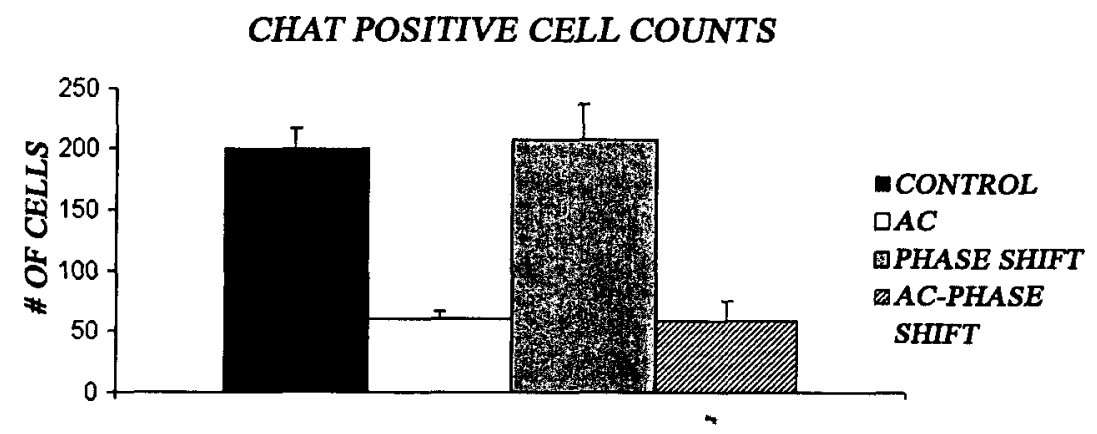

Figure 7.5: Choline acetyltransferase (ChAT) positive cell counts show a clear depletion in $\mathrm{AC} \mathrm{(71 \% )} \mathrm{and} \mathrm{AC-phase} \mathrm{shift} \mathrm{(71 \% )} \mathrm{rats} \mathrm{compare} \mathrm{to} \mathrm{Phase} \mathrm{shift} \mathrm{and} \mathrm{Control} \mathrm{rats.} \mathrm{Data}$ are given as means $+/$ - SEM. 


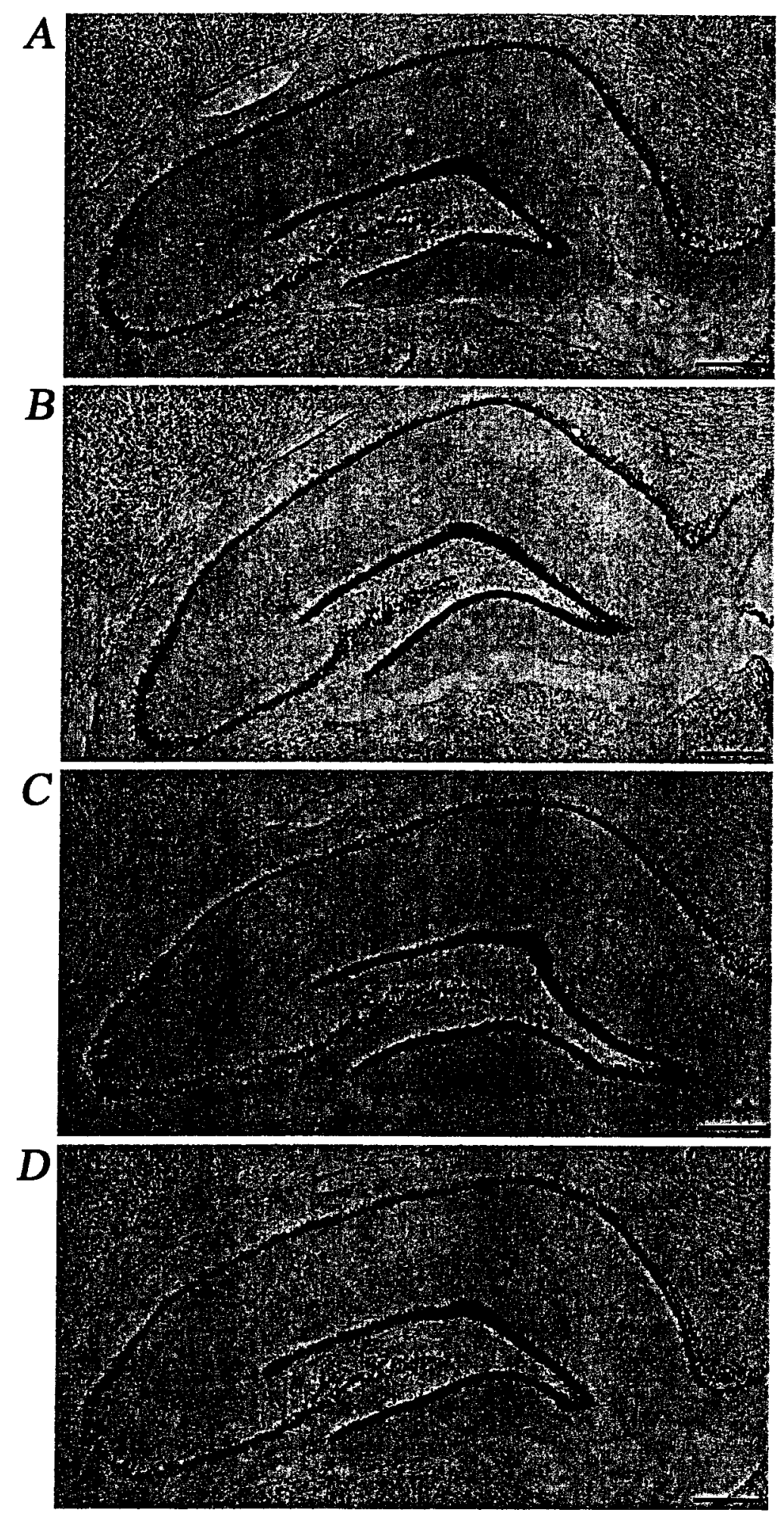

Figure 7.6: Photomicrographs (5x) of Cresyl violet stained hippocampal sections from a Control rat (A), a Phase shift rat (B) and an AC-phase shift rat (C) showing no obvious damage. Scale bars, $1.0 \mathrm{~mm}$. 
Lesioned animals had areas of necrotic cell death in the MS/VDB surrounding the injection site. This non specific damage has been reported previously (Frick et al. 2004) and work in our laboratory has shown no correlation between lesion size (due to focal necrosis following infusion of the toxin) and water maze or fear conditioning performance (data not shown). Analysis of Cresyl violet stained slides under a 10x objective did not reveal any changes in hippocampal structure (Figure 7.6).

\section{Discussion}

Here I show that a specific cholinergic depletion of the medial septum has no effect on the rats' ability to entrain to light, or to re-entrain following phase shifting. Moreover, the combination of cholinergic depletion and phase shifting did not affect learning and memory on the tasks used here. All four groups were able to learn the location of both platforms during water maze training and successfully demonstrate fear conditioning to both tone and context. There was a slight increase in the amount of freezing observed in the groups that had been phase shifted during contextual fear conditioning. This was likely due to the stress of the phase shift (Sakellaris et al., 1975) as increased freezing during this task has been reported previously in rats treated chronically with corticosterone (CORT; Skorzewska et al., 2006). There were no differences between the two phase shifted groups, suggesting that the cholinergic depletion did not modify this behaviour. In addition, all four of the groups exhibited increased freezing when returned to the original context demonstrating that they were able to learn this task. Histological analyses of the lesion confirmed a specific loss of cholinergic neurons in the MS/VDB and a corresponding reduction of $\mathrm{AChE}$ staining in the hippocampus. 
The lack of impairment in spatial memory in my combined group (cholinergic depletion and phase shifting) was an unexpected finding. Specific depletion of medial septal cholinergic neurons does not impair learning and memory on either water maze (Baxter et al., 1995; Bizon et al., 2003; Frielingsdorf et al., 2006) or fear conditioning (cued and contextual) tasks (Frick et al., 2004; Craig et al., 2008); however, previous work in our laboratory has suggested that this depletion increases hippocampal vulnerability to secondary insults, such as stress, non-convulsive seizures and subthreshold stroke (Craig et al., 2008; Craig et al., submitted). When these secondary risk factors are presented at sub-threshold levels, there is no effect on learning and memory, but in rats with a cholinergic depletion of the medial septum, these combinations lead to impairment on the spatial version of the water maze task in the absence of a general performance deficit (Craig et al., 2008; Craig et al., submitted). I expected a similar pattern of results to appear with the combination of phase shifting and cholinergic depletion. The observation that this combination does not impair learning, stresses the importance of recognizing what risk factors, and importantly, which combination of risk factors play a role in the development of dementia.

\section{Cholinergic projections and circadian rhythms}

The discovery of cholinergic projections from the basal forebrain to the SCN led to a number of experiments exploring the role of these-projections on circadian rhythmicity and sleep (Bina et al., 1993, 1997). Most of these experiments have used cholinergic agonists and antagonists to suggest a role for $\mathrm{ACh}$ in mediating the phase shifting response to light (Zatz and Herkenham, 1981; Murakami et al., 1984; Zhang et al., 1993; Ferguson et al., 1999). To the best of my knowledge there have been few 
studies exploring the effects of cholinergic depletion of the basal forebrain on these functions. Bassant et al. (1995) and Kapas et al. (1996) both reported transient sleep disturbances following an intraventricular infusion of IgG SAP that destroyed the majority of cholinergic neurons in the basal forebrain. Erhardt et al. (2004) looked at circadian rhythms following interventricular infusion of IgG SAP and found no effect on the ability of the rat to entrain to a 12 hour LD schedule, but depleted rats demonstrated abnormal responses to light pulses. This is in agreement with the findings of Beule and Amir (2002) who found no change in photic entrainment after intra-ventricular or intraSCN infusions of IgG SAP. To further examine the contribution of specific basal forebrain nuclei, Madeira et al. (2004) used quinolinic acid to lesion the nucleus basalis in rats and found decreased synthesis and expression of neuropeptides [vasopressin (VP) and vasoactive intestinal peptide (VIP)] in the SCN. However, other groups have reported no change in VIP expression following intraventricular infusion of IgG SAP (Moga, 1998; Erhardt et al., 2004).

This is the first study designed to examine the effects of a specific MS/VDB cholinergic lesion on measure of circadian rhythmicity. There are fewer projections from the MS/VDB to the SCN than observed in the NB, and my results clearly show these few projections are not essential to the rats' ability to entrain to light, or to adapt to a strenuous phase shifting schedule. One shortcoming of the current study is the lack of data recorded during constant darkness which would reveal changes in the free running period that can be "masked" in the presence of a strong zeitgeber such as light. Despite this limitation, changes in the free running period are rarely observed following cholinergic depletion (Endo et al., 2001; Beaule and Amir, 2002) and I would not expect 
to see changes in period length here. Taken together these experiments suggest a role for cholinergic projections in modulating circadian rhythms, but more studies are required to describe the exact nature of this relationship.

\section{Circadian disruption and cognition}

This is the first study to look at the behavioural effects of phase shifting in rats following cholinergic depletion. The phase shifting schedule used here does not affect learning and memory when rats are trained and tested ten days post phase shift; however, if rats are trained on the water maze during the six days of phase shifting and tested on a probe trial, without the platform, 17 days later, they exhibit impaired memory for the platform location, suggesting a consolidation deficit (Deven et al., 2001). Furthermore, repeated phase shift and re-entrainment sessions have been found to impair water maze performance, but not fear conditioning (Craig and McDonald, 2008). Fekete et al. (1985) found that there was a discrepancy between time taken to re-entrain following a phase shift, and the length of time required for learning and memory processes to recover. Three days were required for the acquisition of new information following a single phase, while re-entrainment did not occur till later. Tapp and Holloway (1981) reported retrograde amnesia for a task learnt prior to a single phase shift. Retention of this memory was impaired both 24 hours and seven days after the phase shift. Taken together these studies suggest that a threshold exists for the type, timing and duration of circadian disruption in relation to learning.

Similar findings have been reported in humans following circadian disruption due to shift work or jet lag. Rouch et al. (2005) have examined the effects of circadian disruption due to shift work in humans and reported that short term shift work has few 
effects on cognition; however, long term shift work is correlated with impaired cognition. Cho and colleagues (Cho, 2000; Cho et al., 2001) found similar cognitive impairments in flight attendants suffering from chronic jet lag. These individuals also had increased levels of cortisol and atrophy of the right temporal area suggesting possible mechanisms for the observed impairment following chronic circadian disruption.

\section{Circadian rhythms and cholinergic depletion in Alzheimer's disease}

Cholinergic depletion has been found early on in the development of $\mathrm{AD}$ (Bowen et al., 1992; Perry et al., 1992; Schliebs and Arendt, 2006) and reduced melatonin levels have been proposed as an early marker of AD (Liu et al., 1999; Wu et al., 2003). These studies suggest that an early interaction between these two factors may play a role in the development of the disorder. The circadian disruption observed in $\mathrm{AD}$ patients has been linked with the severity of their dementia and with the integrity of the SCN (van Someran et al., 1996). Loss of cholinergic projections to the SCN could accelerate the appearance of circadian disruption in these patients leading to increases in the severity of dementia. Apart from affecting circadian rhythmicity, cholinergic projections to the SCN could have an indirect effect on learning and memory through alterations in VP expression. Madeira et al. (2004) has reported a significant decline in VP synthesis and expression following a lesion of the cholinergic neurons of the NB. Interestingly, VP has been implicated in learning and memory, specifically in șocial interactions, but also in a number of behavioural task such as radial maze, passive avoidance and water maze (for a review see Caldwell et al., 2008). It would be interesting to explore whether there are decreases in VP expression following specific cholinergic depletion of the MS/VDB. I did observe any change in circadian rhythmicity or learning and memory following this 
lesion, suggesting MS/VDB projections are not essential for the modulating effects of $\mathrm{ACh}$ in the SCN.

\section{Multiple combinations of co-factors theory of aging}

I have conducted a number of studies that provide support for the multiple combinations of co-factors theory of aging proposed by McDonald (2002). This theory describes passive and active risk factors that can have negative effects on the hippocampus. Passive factors (such as cholinergic depletion, stress, phase shifting) act to increase the vulnerability of hippocampal neurons, while active factors (stroke, seizures, head trauma) can directly damage or kill hippocampal neurons. I have explored the histological and cognitive effects of both passive-active combinations of risk factors and passive-passive combinations of risk factors. I found consistent impairment in spatial learning and memory as tested in the water maze task, but no impairment on tests of nonspatial learning such as cued water maze training, or fear conditioning (Craig et al., 2008; McDonald et al., 2008; Driscoll et al., 2007; Craig et al., submitted). One concern with the above mentioned studies is the suggestion that the observed impairment is simply the result of an additive effect of two insults on the brain, irrespective of what those insults may be. The current study argues against that explanation as the passive-passive combination used here has no effect on measures of learning and memory, or on circadian rhythmicity and hippocampal integrity that could develop into cognitive deficits over time. If a simple "two-hit" model could explain the mnemonic deficits previously observed, I would expect to see impairment in the current study. 


\section{Conclusion}

In conclusion, I have shown that specific cholinergic depletion of the medial septum does not effect entrainment to a 12 hour LD cycle, or the ability of the rat to reentrain following phase shifting. In addition, the combination of these two factors (cholinergic depletion and phase shifting) does not impair learning and memory on the tasks used here. This was in contrast to previous studies examining the effects of various risk factors for $\mathrm{AD}$ following cholinergic depletion and finding deficits in hippocampal based learning and memory in rats subjected to two factors. Future studies will look at the effects of repeated phase shifting sessions to determine whether the previously reported chronic phase shifting deficit occurs earlier in cholinergic depleted rats due to increased hippocampal vulnerability. 


\section{Chapter 8}

General Discussion 
Two basic questions that drive Alzheimer's disease research are: What causes Alzheimer's disease? And what can be done to prevent or cure it? Without an understanding of the accurate etiology of this disorder, we will be no closer to developing an effective cure than we are today. The multiple combinations of co-factors theory of Alzheimer's disease appears to be a plausible model that could open the door to novel treatment strategies. I have used this model, in combination with the original cholinergic hypothesis of AD to propose a "new" cholinergic hypothesis that is presented in this thesis. This new version takes into account recent findings from the literature and my reports of increased hippocampal vulnerability to secondary insults following cholinergic depletion of the medial septum. I will first summarize the experimental results from this thesis (Table 8.1) and discuss some potential mechanisms that could explain my results. I will then present my 'new' version of the cholinergic hypothesis and how it relates to the field of $\mathrm{AD}$ research today. Finally I will discuss some of the implications for treatment that arise from this model and present some directions for future study.

\section{Sub-threshold models of risk factors for Alzheimer's disease}

There are many animal models of brain injury in use today, but most of these model acute injuries that result in both functional and pathological impairment. I was interested in the cumulative effects of subtle damage that could better model the 'damage' that the brain is habitually subjected to and compensates for on a regular basis in the absence of noticeable impairment. In order to do this, I developed four novel models of sub-threshold risk factors for future use in studying the contribution of different risk factors to $\mathrm{AD}$ (e.g., stress, seizures, stroke and circadian disruption). 
The first three risk factors (stress, seizures and stroke) have been previously shown to damage hippocampal neurons and produce behavioural impairment on learning and memory tasks. For my purposes, it was important to develop procedures that could act via the same mechanism (elevated corticosterone (CORT), epileptiform activity, ischemic damage), but not affect behaviour as tested on a hippocampal dependent version of the water maze. Chronic stress was modeled using a restraint stress procedure that varied the duration of the stress each day for two weeks. This procedure reliably elevated CORT levels, but did not impair learning and memory. Non-convulsive seizure activity was induced by systemic injection of a low dose of kainic acid that was able to cause local epileptiform activity in the brain without progressing to convulsive seizures. This procedure did not lead to cognitive deficits or hippocampal damage. Finally I found that a low dose of the vasoconstrictor Endothelin-1 infused directly into the hippocampus was able to mimic the minor subcortical damage resulting from a 'silent' stroke, in the absence of behavioural impairment.

My fourth risk factor, circadian disruption, had not been previously shown to disrupt learning and memory when presented well before initial training, but reports from the human literature suggests that prolonged circadian disruption may be associated with memory impairment and hippocampal atrophy. Here I presented an acute and a chronic version of a circadian 'challenge' that in its acute version'does not affect learning and memory. However, when rats were subjected to the chronic phase shifting procedure, they demonstrated impaired spatial learning and memory in the water maze, but had no difficulty learning a fear conditioning task. From these results I chose to use the acute phase shifting procedure as a sub-threshold model of circadian disruption. 


\section{Cholinergic depletion combined with an active risk factor}

According to McDonald's (2002) multiple combination of co-factors theory of $\mathrm{AD}$, the presentation of a passive risk factor prior to the occurrence of an active risk factor, both targeted at the hippocampus, should result in impaired hippocampal learning and memory, most likely due to increases in the severity of neuronal damage. I did observe learning and memory impairment in rats subjected to both a passive and an active factor; however, I did not observe increased hippocampal damage. My third experiment combined cholinergic depletion of the medial septum (a passive factor) with seizures (an active factor). I observed a trend towards increased seizure severity in the combined group and reported impairment on the spatial version of the water maze. However, there were no differences in hippocampal pathology in the seizure-only or combined groups.

My fourth experiment also supported this prediction. I combined cholinergic depletion (a passive factor) with stroke (an active factor) and reported impaired spatial learning and memory in the combined group. Volumetric analysis of the hippocampus revealed a moderate amount of damage due to stroke, but this amount was comparable between the stroke-only and combined group. Taken together these two experiments confirm that a passive factor combined with an active factor can impair hippocampal learning and memory. Surprisingly this impairment was not accompanied by an increase in pathology suggesting that more subtle interference in hippocampal processing is likely responsible for the cognitive deficit.

\section{Cholinergic depletion combined with a passive risk factor}

The combination of two passive factors was not expected to impair learning and memory on either task. This combination should have increased vulnerability of the 
hippocampus, but not to the extent where cognition would be affected. My fifth experiment looked at cholinergic depletion of the medial septum (a passive factor) combined with chronic stress (a $2^{\text {nd }}$ passive factor). Surprisingly, this combination impaired performance on the spatial version of the water maze, but did not affect fear conditioning to tone, or to context. Moreover, there were no differences in overall levels of CORT in the stress-only or combined group, and once again, there was no obvious damage to hippocampal neurons suggesting an alternate mechanism was responsible for the impairment.

My sixth experiment combined cholinergic depletion (a passive factor) with circadian disruption (a $2^{\text {nd }}$ passive factor) and found no effect of this combination on measures of circadian rhythmicity, or on learning and memory as tested on the spatial water maze, and fear conditioning. This was a surprising finding as I had previously attributed the impairment following chronic phase shifting to the stressfulness of the procedure. It follows that if cholinergic depletion and stress impair memory, then cholinergic depletion followed by phase shifting (a presumably stressful event) should have a similar effect. The absence of such an effect points to several alternative explanations for my chronic phase shifting data, and more importantly demonstrates that this the co-factor model is not simply a "two-hit" hypothesis, but that the nature of the 'hits' is important. It is possible that the single session of phase shifting used here was not close enough to threshold (four sessions) to produce impairment, but two or three sessions may impair hippocampal memory in the depleted rat, similar to the impairment observed following four sessions of phase shifting (Craig and McDonald, 2008). Alternatively the presence of a running wheel (an intervention shown to enhance spatial 
memory and increase the expression of neurotrophic factors; Adlard et al, 2004 ;

Vaynmann et al., 2004; Alaei et al., 2007) was able to compensate for the combination of the two risk factors resulting in the lack of impairment in this group.

Table 8.1: Summary of behavioural testing and gross hippocampal pathology

\begin{tabular}{|c|c|c|c|c|c|}
\hline & $\begin{array}{c}\text { Effect of } \\
\text { secondary } \\
\text { risk factor } \\
\text { in depleted } \\
\text { rats } \\
\end{array}$ & $\begin{array}{l}\text { Hippocampal } \\
\text { Damage }\end{array}$ & $\begin{array}{l}\text { Spatial } \\
\text { water } \\
\text { maze }\end{array}$ & $\begin{array}{c}\text { Fear } \\
\text { conditioning }\end{array}$ & $\begin{array}{c}\text { Visible } \\
\text { water maze }\end{array}$ \\
\hline $\begin{array}{c}\text { Cholinergic } \\
\text { depletion } \\
\text { and } \\
\text { seizures }\end{array}$ & $\begin{array}{c}\text { Trend } \\
\text { towards } \\
\text { increased } \\
\text { seizure } \\
\text { severity }\end{array}$ & $\begin{array}{c}\text { No gross } \\
\text { hippocampal } \\
\text { damage }\end{array}$ & $\begin{array}{l}\text { Combined } \\
\text { group was } \\
\text { impaired on } \\
\text { all stages }\end{array}$ & $\mathrm{N} / \mathrm{A}$ & $\begin{array}{c}\text { No } \\
\text { difference } \\
\text { between } \\
\text { kainic-only } \\
\text { and } \\
\text { combined } \\
\text { rats }\end{array}$ \\
\hline $\begin{array}{c}\text { Cholinergic } \\
\text { depletion } \\
\text { and stroke }\end{array}$ & $\begin{array}{c}\text { No } \\
\text { difference in } \\
\text { extent of } \\
\text { hippocampal } \\
\text { damage }\end{array}$ & $\begin{array}{c}\text { Moderate } \\
\text { hippocampal } \\
\text { damage in both } \\
\text { groups }\end{array}$ & $\begin{array}{l}\text { Combined } \\
\text { group was } \\
\text { impaired on } \\
\text { all stages }\end{array}$ & N/A & $\begin{array}{c}\text { No } \\
\text { difference } \\
\text { between } \\
\text { combined } \\
\text { rats and } \\
\text { controls } \\
\end{array}$ \\
\hline $\begin{array}{l}\text { Cholinergic } \\
\text { depletion } \\
\text { and stress }\end{array}$ & $\begin{array}{l}\text { Similar } \\
\text { elevation of } \\
\text { CORT }\end{array}$ & $\begin{array}{c}\text { No gross } \\
\text { hippocampal } \\
\text { damage }\end{array}$ & $\begin{array}{l}\text { Combined } \\
\text { group was } \\
\text { impaired on } \\
\text { all stages }\end{array}$ & $\begin{array}{c}\text { No } \\
\text { impairment }\end{array}$ & N/A \\
\hline $\begin{array}{c}\text { Cholinergic } \\
\text { depletion } \\
\text { and } \\
\text { circadian } \\
\text { disruption } \\
\end{array}$ & $\begin{array}{c}\text { Similar } \\
\text { responses to } \\
\text { phase } \\
\text { shifting }\end{array}$ & $\begin{array}{c}\text { No gross } \\
\text { hippocampal } \\
\text { damage }\end{array}$ & $\begin{array}{c}\text { No } \\
\text { impairment }\end{array}$ & $\begin{array}{c}\text { No } \\
\text { impairment }\end{array}$ & N/A \\
\hline
\end{tabular}

The cholinergic hypothesis of Alzheimer's disease

It is now 26 years after the cholinergic hypothesis of geriatric memory dysfunction was first proposed by Bartus et al. (1982). After an initially promising start, recent studies have called many of the previous findings supporting this theory into question and it is no longer widely believed that the cholinergic depletion alone is 
responsible for causing $\mathrm{AD}$. This has been clearly demonstrated by the lack of efficacy of acetylcholinesterase inhibitors (AChEIs) in clinical trials (Mohs et al., 2001; Winblad et al., 2001) and the finding that cholinergic depletion does not result in severe memory deficits in rats (Mesulam, 2004; Parent and Baxter, 2004). However, I am now beginning to revisit the role of the cholinergic depletion from a different angle. Using the co-factors model as a theoretical framework, and based on the findings that a loss of cholinergic neurons and/or activity is an early event in the development of $\mathrm{AD}$ (Perry et al., 1992; Geula et al., 2008), I propose that this depletion leads to a reduction in the ability of the brain to compensate for secondary insults. This view of the cholinergic depletion differs from that proposed originally by Bartus et al. (1982) who saw the depletion as being causative on its own. My proposed model is also distinct when compared to more recent theories suggesting that the reduction in cholinergic activity results from the accumulation of $A \beta$ and initiates an unfavorable feedback loop leading to the cognitive impairment seen in $\mathrm{AD}$ (Auld et al., 1998; Auld et al., 2002). In my model, this depletion would not be causative, or affect learning and memory on its own, as supported by literature in rodents, non-human and human primates, but would reduce the ability of the brain to compensate for the accumulation of risk factors that occur with increasing frequency during the aging process.

\section{A neuroprotective or compensatory role for acetylcholine in response to injury}

The cholinergic system has been implicated in mediating plasticity in the brain in response to experience or injury. There are three lines of evidence that support this hypothesis. First, there are numerous reports of the beneficial effects of cholinergic agonists on enhancing recovery and minimizing neuronal damage in various injury 
models. Secondly, cholinergic depletion has been found to impair experience-dependent plasticity in the cortex and hippocampus and similar mechanisms are involved in mediating recovery from injury. Thirdly, acetylcholine modulates the expression of neurotrophic factors such as BDNF that play a major role in neuronal survival in adulthood.

Beneficial effects of nicotine and cholinergic agonists on recovery from injury and neurodegenerative disorders

A neuroprotective role for the cholinergic agonist nicotine has been proposed and this role of the cholinergic system could begin to explain my findings (Borlongan et al., 1995; Takada et al., 2003; Gonzalez et al., 2006; Mudo et al., 2007). The neuroprotective effects of the cholinergic system are largely mediated through stimulation of nicotinic AChRs, either directly through nAChR agonists (e.g., nicotine) or indirectly via AChEIs (e.g., donepezil) that increase the availability of $\mathrm{ACh}$ that can then bind to nAChRs. Muscarinic agonists may also be a promising approach for treating the neuropathology and cognitive decline observed in $\mathrm{AD}$ and aging (Wanibuchi et al., 1994; Suzuki et al., 1995; Suzuki et al., 1995b; Fisher, 2000) as well as stroke (Yamaguchi et al., 1995); however, their ability to successfully treat a wide variety of neurodegenerative disorders has not been demonstrated. In contrast to the scarcity of reports on muscarinic agonists, the ability of nicotine to produce neuroprotective effeots in a wide variety of neurodegenerative models has been well documented. In vitro studies show nicotine can protect cortical and hippocampal neurons from NMDA-mediated excitotoxicity (Akaike et al., 1994; Dajas-Bailador et al., 2000) and cultured hippocampal neurons from $\beta A$ induced neurotoxicity (Zamani et al., 2001). Moreover, nicotine has been shown to 
reduce neuronal damage and ameliorate behavioural deficits in animal models of stroke (Nanri et al., 1998; Fujiki et al., 2005; Gonzalez et al., 2006), Parkinson's disease (Maggio et al., 1998; Costa et al., 2001), Huntington's disease (Tariq et al., 2005), traumatic brain injury (Verbois et al., 2003) as well as kainic acid induced seizures (Borlongan et al., 1995; Shin et al., 2007). Taken together, these studies suggest an important role for nicotine acting via the $\mathrm{nAChR}$ in compensating for damage. There is a reduction in the number of $\mathrm{nAChRs}$ in the hippocampus and cortex of patients diagnosed with $\mathrm{AD}$ which may explain their difficulty recovering from secondary risk factors (Nordberg, 2001).

Impaired experience-dependent plasticity in cholinergic depleted animals

Experience-dependent plasticity refers to changes in the brain in response to a specific event or experience. For example, acquisition of a skilled reaching task in rodents, humans and non-human primates has been associated with an expansion of the motor map, specifically in the forelimb area. Specific lesions of the basal forebrain cholinergic neurons in adult rats have been shown to decrease this normal expansion of the motor map during skilled reaching training (Conner et al., 2003). A similar role for $\mathrm{ACh}$ is apparent in the somatosensory barrel cortex of rodents, where cholinergic depletion reduces experience-dependent plasticity in this area (Baskerville et al., 1997; Zhu et al., 1998). The reverse can also be shown as stimulating cholinergic neurons of the nucleus basalis enhances experience-dependent plasticity in the auditory cortex (Kilgard and Merzenich, 1998). Experience-dependent plasticity can also be associated with changes in dendritic branching and spine density, with greater branching often associating with greater capacity for change. Damaging the nucleus basalis has been 
found to reduce branching of pyramidal neurons in the frontal cortex a finding that could explain the reduced plasticity in cortical neurons (Harmon and Wellman, 2003; Works et al., 2004). Moreover, neonatal depletions have been shown to decrease dendritic branching in cortical neurons examined in adulthood (Robertson et al., 1998; Sherren and Pappas, 2005).

There have been fewer studies looking at $\mathrm{ACh}$ and hippocampal plasticity, but those that do appear to follow a similar pattern of results. Long-term potentiation (LTP) is a model of neural plasticity and natural mechanisms similar to LTP are thought to be the basis for learning and memory in the brain. Indeed, by blocking or enhancing LTP expression, animals tend to show memory deficits or improvements respectively (Stevens, 1998; Martin et al., 2000). Some studies have suggested that the ability to induce hippocampal LTP in rats following cholinergic depletion is reduced (Motooka et al., 2001; but see Jouvenceau et al., 1996). In addition, nicotine has been shown to enhance the ability to induce LTP, likely through stimulation of nAChRs (Matsuyama et al. 2000; Fujii et al., 2001; Ji et al., 2001; McGehee, 2002). These studies suggest that both cortical and hippocampal ACh projections are important for experience-dependent plasticity and learning. Effects of ACh on neurotrophin expression and neurogenesis

One of the proposed mechanisms for the effects of ACh on plasticity and response to injury is its ability to modulate the expression of neurotrophin factors and neurogenesis. Neurotrophins such as brain derived neurotrophic factor (BDNF), nerve growth factor (NGF) and fibroblast growth factor (FGF-2) play an important role in neuronal growth and survival in adulthood. Interestingly, all of these neurotrophins are 
increased following treatment with nicotine (Belluardo et al., 1998; Maggio et al., 1998; Kenny et al., 2000; Martinez-Rodriguez et al., 2003) and Maggio et al. (1998) found that the beneficial effects of nicotine in the Parkinson's disease mouse model were correlated with increases in FGF-2 and BDNF. There are no changes in basal levels of hippocampal BDNF or NGF mRNA one to four weeks after cholinergic depletion in rats (Yu et al., 1995; Gu et al., 1998), but at later time points significant decreases were reported in the hippocampus and cortical areas (Kokaia et al., 1996; Ferencz et al., 1997). Interestingly, this depletion also eliminated the seizure mediated increase in BDNF and NGF suggesting a role for acetylcholine in mediating the endogenous response of neurotrophins to injury (Kokaia et al., 1996; Ferencz et al., 1997).

Hippocampal neurogenesis has also been suggested to play a role in learning and memory and decreased neurogenesis has been correlated with poor aging in rats (Driscoll et al. 2006). Enhancing neurogenesis through enriched environment, exercise or specific drugs (anti-depressants) has been shown to improve memory and performance on hippocampal dependent tasks (Nilsson et al., 1999; van Praag et al., 1999; Malberg et al., 2000). Conversely, blocking neurogenesis through radiation, adrenalectomy, or drugs targeting new neurons, has been shown to impair performance in a number of tasks dependent on the hippocampus (Shors et al., 2001; Snyder et al., 2005; Spanswick et al., 2007). Following cholinergic depletion rates of neurogenesis are significantly decreased (Cooper-Kuhn et al., 2004; Mohapel et al., 2005).

Taken together these studies have demonstrated the importance of $\mathrm{ACh}$ in experience-dependent plasticity and response to injury, most likely through its effects on neurotrophins, (especially BDNF), neurogenesis, and dendritic branching. 


\section{A look at my results using the 'new' cholinergic hypothesis}

Using the hippocampus as the initial site of AD memory loss and pathology, I proposed that the normal role of ACh is to mediate a number of compensatory mechanism (neurogenesis, neurotrophin factors, changes in dendritic branching) that participate in learning and memory and functional recovery from injury. An explanation of my results using the 'new' cholinergic hypothesis is depicted in Figure 8.1. In a normal, healthy individual a sub-threshold injury often goes unnoticed as ACh-mediated compensatory mechanisms are sufficient to compensate for the damage (8.1A). Unfortunately, following a major insult such as stroke these compensatory mechanisms are not sufficient to fully overcome the damage from stroke leading to memory impairment (8.1B). However, in the absence of cholinergic projections to the hippocampus, a minor sub-threshold insult (mild ischemia, elevated glucocorticoids, epileptiform activity) can lead to memory impairment due to the loss of these AChmediated compensatory mechanisms as was shown in the current series of studies (8.1C). This model also provides a possible explanation for the lack of impairment observed following circadian disruption and cholinergic depletion. In this situation, animals had access to running wheels, an intervention that has been shown to enhance neurogenesis, increase the secretion of neurotrophic factors (e.g., BDNF, FGF, NGF), enhance dendritic branching, and has been correlated with improved functional recovery on behavioural tasks. At least one of these compensatory mechanisms (increased BDNF) has been shown to be increased by wheel running independent of the septo-hippocampal cholinergic system (Berchtold et al., 2002). Increased BDNF has been shown to enhance learning and memory and promote recovery from stroke (Ferrer et al., 2001; Schäbitz et al., 2004; 
Vaynmann et al., 2004). This suggests that in the absence of ACh-mediated compensation, wheel running activity may have provided similar support to the hippocampus through increases in BDNF and no impairment was observed (8.1D).
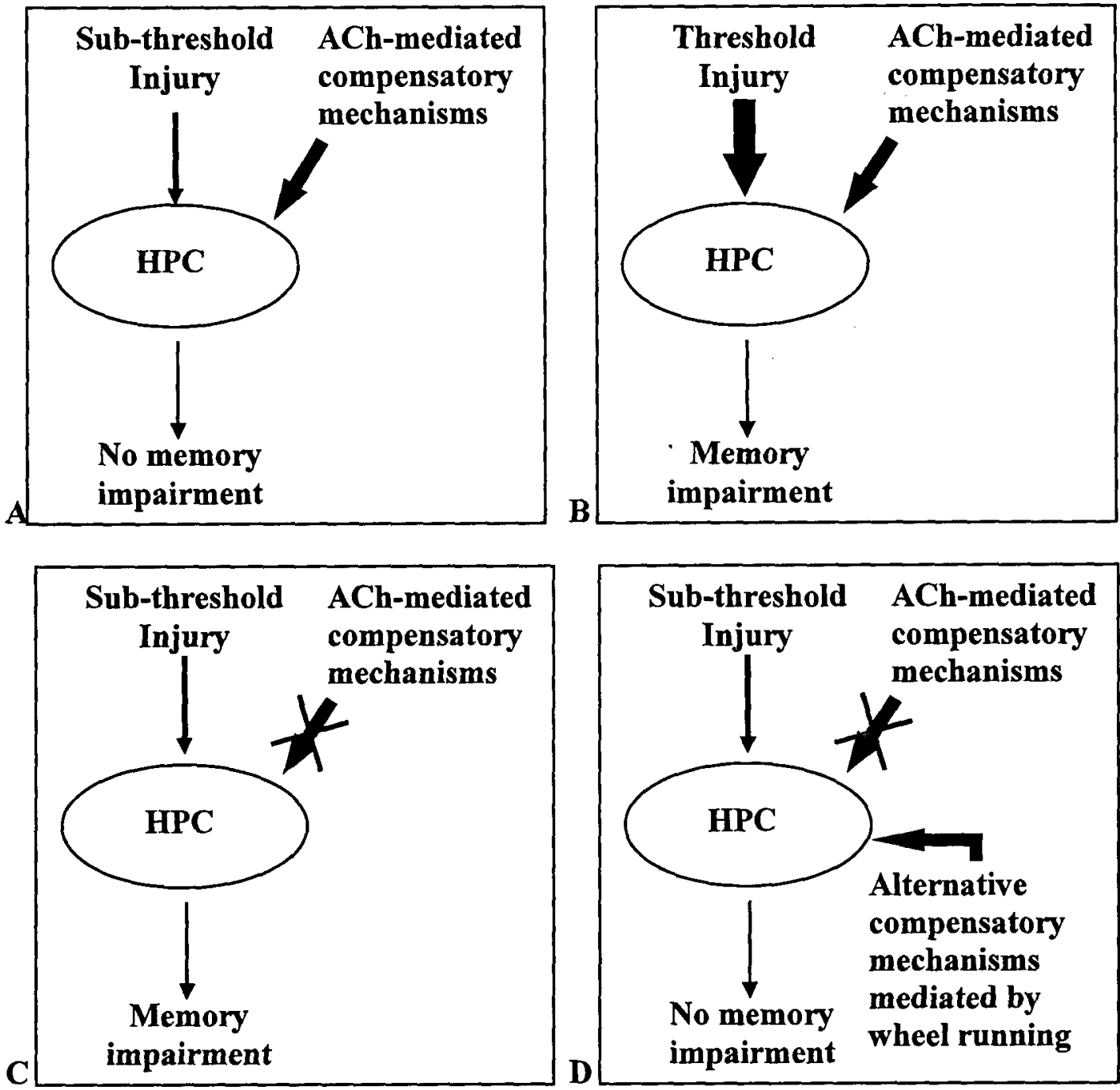

Figure 8.1: Explanation of presented results using the 'new' cholinergic hypothesis

This model suggests a potential role for $\mathrm{ACh}$ in mediating functional recovery from sub-threshold insults targeting the hippocampus through its effects on BDNF. Using this theoretical framework the development and progression of $\mathrm{AD}$ can be explained. The earliest forms of memory to be lost in $\mathrm{AD}$ (e.g., episodic, spatial, declarative) are 
hippocampal dependent. This loss is most likely because of the exceptional sensitivity of the hippocampus to exogenous damage (e.g., ischemia, excitotoxicity, glucocorticoids). However, the cholinergic depletion reported in aged humans and in those with $\mathrm{AD}$ is global in nature, affecting both hippocampal and cortical areas. As aging progresses, individuals are subjected to potentially damaging risk factors that initially affect the hippocampus, and over time effect depleted cortical regions as well, resulting in the more global memory loss observed in the late stages of $\mathrm{AD}$. Interestingly, cholinergic neurons in the striatum remain relatively intact in $\mathrm{AD}$, memory tasks dependent on the striatum are not nearly as severely affected in $\mathrm{AD}$ patients (Carlesimo and Oscar-Berman, 1992).

\section{Multiple combinations of co-factors model of Alzheimer's disease}

The present data, combined with previous work (Driscoll et al., 2007; McDonald et al., 2008) has provided support for the co-factor theory of $\mathrm{AD}$. Five out of the six combinations of co-factors examined on measures of learning and memory and hippocampal damage, resulted in hippocampal dependent memory impairment. Interestingly only two combinations [aging and stroke (Driscoll et al., 2007) and stress and stroke (McDonald et al., 2008)] reported an increase in hippocampal damage following the second factor that was likely responsible for the cognitive deficit. The remainder of the studies found no sign of gross hippocampal damage that could explain the impairment. Taken together, these studies stress the importance of using behavioural assessment in conjuncture with histological measures when assessing with animal models of disease, and tracking recovery from injury, or response to treatment.

Many reports in the literature focus solely on decreased lesion size as an end point measure of a successful treatment without conducting careful behavioural analysis. 
Histological measures are essential for basic research and for understanding mechanisms; however, from a clinical perspective, functional recovery is the desired outcome, regardless of what effect a treatment has on overall lesion size. This is illustrated here as I clearly show that a cognitive deficit due to $\mathrm{AD}$ risk factors can be apparent in the absence of gross hippocampal damage. These findings are particularly relevant to $\mathrm{AD}$ as the amount of variability between aged individuals is extreme and there is no clear-cut correlation between neuropathology and the severity of cognitive decline or dementia. Many non-demented aged individuals have numerous plaques and tangles and there are just as many demented individuals that show little pathology. The co-factor model proposes a way to encompass all varieties of cognitive decline by looking carefully at the risk factors involved and forming specific subgroups, for which customized treatments can be designed.

\section{Treatment implications}

The subgroup described in this thesis can be defined by its early cholinergic depletion and associated decrease in compensatory mechanisms as the driving force behind the progression of this disorder. I do not see this decline in ACh levels as a causal factor, but instead, as an important risk factor that leads to the increased susceptibility of an individual to develop $\mathrm{AD}$. This subgroup would have several proposed treatment options focused on prevention, or early intervention thãt would enhance available $\mathrm{ACh}$, and/or increase the activity of alternative compensatory pathways. Current treatments for $\mathrm{AD}$ using AChEIs are not initiated until clinical signs of memory loss are present (when neuropathological damage is likely present) and reports have shown this is too late. When $\mathrm{AChEIs}$ are prescribed early on in $\mathrm{AD}$, there is more success in slowing the progression 
of this disorder, but an overall decline in cognitive function still occurs in all patients (Doody et al., 2001).

Early treatment of dementia with cholinomimetics was first proposed by Bartus et al (1980, 1982), but few studies have explored the preventative effects of these drugs. Current AChEIs have too many side effects to make them desirable or feasible as preventative medicine, but choline and lecithin (precursors to $\mathrm{ACh}$ ) are well tolerated and can be readily obtained through specific foods (e.g., eggs, beef, liver, nuts, broccoli, potatoes). Studies examining the effects of these ACh precursors have found no improvements in $\mathrm{AD}$ patients on the whole, but dietary choline was found to prevent the age related impairments in passive avoidance learning in mice (Bartus et al., 1980). The role for ACh presented here would predict that the choline supplement occurred too late in the human subjects (i.e. once $\mathrm{AD}$ symptoms were already present) and that early treatment with ACh precursors could stave off this disorder. A choline rich diet could protect against subsequent risk factors and prevent the changes in hippocampal processing that could eventually lead to mnemonic impairment and the development of $\mathrm{AD}$ neuropathology. Alternatively, regular testing of ACh levels in the 40-60 year old population could be helpful to initiate preventative cholinergic therapy as soon as a decline is detected. This is the age when the difference between the dramatic cholinergic depletion observed in $\mathrm{AD}$ patients and the normal age-related decline in $\mathrm{ACh}$ levels becomes most evident (Perry et al., 1992). A treatment designed to counteract the cholinergic decline as soon as it occurs may be able to prevent or reduce the onset and severity of $\mathrm{AD}$ by prolonging the ability of $\mathrm{ACh}$ to provide neuroprotection in the aging brain. 
The second area for treatment focuses on enhancing alternative compensatory mechanisms in the brain that could replace the deficient ACh-mediated compensation. Environmental enrichment, exercise, and training have all been shown to have positive effects on measures of plasticity (e.g., neurogenesis, neurotrophin expression, dendritic morphology) and enhance recovery in animal models (Kolb and Gibb, 1991; Kolb et al., 1998; Nilsson et al., 1999; Ickes et al., 2000; van Praag et al., 2000; Faherty et al., 2003; Mora et al., 2007). In humans, high levels of education, participation in leisure activities and exercise have all been found to decrease one's chances on developing AD (Larson et al., 2006; Stern, 2006; Roe et al., 2007) providing further support for a preventative view of treating $\mathrm{AD}$. The benefit of these interventions is that they would still be effective in the absence of a functional cholinergic system or one that does not respond to cholinergic drugs. Moreover the benefit of exercise has also been demonstrated for reducing stress and cardiovascular disease; both risk factors linked to $\mathrm{AD}$. This model describes how these interventions could be beneficial and attempts to explain the discrepancy between healthy aged individuals with decreased $\mathrm{ACh}$, and aged individuals that develop $\mathrm{AD}$.

\section{Future studies}

I have proposed the co-factor theory of $\mathrm{AD}$ and described an important role for cholinergic depletion in the development of hippocampal dependent memory impairments that are reminiscent of those seen in early AD. Though I was able to replicate the hippocampal specific cognitive impairment, as well as the cholinergic depletion, I did not look for other two classic hall marks of $\mathrm{AD}$ : plaques and tangles. Nonetheless, I have set the stage for future research into this area, and I predict I will detect $\mathrm{AD}$ pathology in the brains of my animals subjected to multiple risk factors. For 
the sake of simplicity, and with the awareness that no single model can adequately mimic all signs of $\mathrm{AD}$, I chose to focus on the hippocampus alone and the early stages of memory loss in $\mathrm{AD}$ to gain a better understanding of the etiology of $\mathrm{AD}$. Future studies will begin to explore some of the ACh-mediated compensatory mechanisms that are reduced in $\mathrm{AD}$. For example these compensatory mechanisms can be explored directly by measuring rates of neurogenesis, levels of neurotrophic factors and changes in dendritic morphology or indirectly by examining the effects of therapies designed to enhance cholinergic activity or increase the levels of beneficial neurotrophins such as BDNF. There are a few specific experiments that would further clarify this model. First, would be to replicate the cholinergic depletion and phase shifting experiment using telemetry to monitor circadian rhythms which would eliminate the confound of wheel running, and secondly, to repeat the combinations that did produce an impairment in rats housed in running wheels to see whether this treatment was effective. Finally, measurement of BDNF levels before and after the secondary risk factor would be essential for describing the role of this neurotrophin with or without ACh. The results of these studies would provide support and additional clarification of the role of $\mathrm{ACh}$ in the development of $\mathrm{AD}$.

\section{Conclusions}

My primary conclusion is that the cholinergic depletion impairs the rats' ability to compensate for secondary insults that target the hippocampus. I present a 'new' version of the cholinergic hypothesis to explain my results and have discussed the importance of prevention and early intervention when treating AD. I remain unaware of the specific underlying mechanism of this effect, but I suggest decreases in BDNF may be important. It is clear from a behavioural perspective that the removal of cholinergic projections to 
the hippocampus severely compromises the ability of the rat to compensate for subsequent injury. Moreover, the lack of impairment in cholinergic depleted rats following phase shifting suggests that the nature of the secondary insult is important for the appearance of the memory deficit. My findings suggest a role for the cholinergic system in providing protection for hippocampal neurons, and/or mediating compensatory mechanisms following injury. 


\section{References}

Abidin I, Yargicoglu P, Agar A, Gumuslu S, Aydin S, Aydin S, Ozturk O, Sahin E (2004) The effects of chronic restraint stress on spatial learning and memory: relation to oxidant stress. Int J Neurosci 114:683-699.

Adkins DL, Voorkies AC, Jones TA (2004) Behavioural and neuroplastic effects of focal endothelin-1 induced sensorimotor cortex lesions. Neuroscience 128:473-486.

Adlard PA, Perreau VM, Engesser-Cesar C, Cotman CW (2004) The timecourse of induction of brain-derived neurotrophic factor $\mathrm{mRNA}$ and protein in the rat hippocampus following voluntary exercise. Neurosci Lett 363, 1:43-48.

Akaike A, Tamura Y, Yokota T, Shimohama S, Kimura J (1994) Nicotine-induced protection of cultured cortical neurons against $\mathrm{N}$-methyl-D-aspartate receptor-mediated glutamate cytotoxicity. Brain Res 644, 2:181-187.

Alaei H, Moloudi R, Sarkaki AR, Azizi-Malekabadi H, Hanninen O (2007) Daily running promotes spatial learning and memory in rats. Pathophysiology 14, 2:105-108.

Alzheimer A, Förstl H, Levy R (1991) On certain peculiar diseases of old age. Hist Psychiatry 2, 5, 1:71-101.

Alzheimer A, Stelzmann RA, Schnitzlein HN, Murtagh FR (1995) An English translation of Alzheimer's 1907 paper, "'Uber eine eigenartige Erkankung der Hirnrinde"". Clin Anat, 8, 6:429-431.

Ambree O, Touma C, Gortz N, Keyvani K, Paulus W, Palme R, Sachser N (2006) Activity changes and marked stereotypic behaviour precedes Abeta pathology in TgCRND8 Alzheimer mice. Neurobiol Aging 27, 7:955-964.

Antle MC, Mistlberger RE (2005) Circadian rhythms. In The Behaviour of the Laboratory Rat, (ed. IQ Whishaw and B Kolb), pp.183-194, Oxford Press, New York."

Antoniadis EA, Ko CH, Ralph MR, McDonald RJ (2000) Circadian rhythms, aging and memory. Behav Brain Res 111:25-37

Aragon CM, Trudeau LE, Amit Z (1990) Stress-ethanol interaction: involvement of endogenous opioid mechanisms. Neurosci BiobehavRev 14, 4:535-541.

Arendt T, Holzer M, Gertz HJ, Brückner MK (1999) Cortical load of PHF-tau in Alzheimer's disease is correlated to cholinergic dysfunction. J Neural Trans 106, 5-6:513523. 
Arriagada PV, Growdon JH, Hedley-Whyte ET, Hyman BT (1992) Neurofibrillary tangles but not senile plaques parallel duration and severity of Alzheimer's disease. Neurology 42:631-639.

Arvidsson A, Kokaia Z, Lindvall O (2001) N-methyl-D-aspartate receptor-mediated increase of neurogenesis in adult rat dentate gyrus following stroke. Eur J Neurosci 14: 10-18.

Auer RN, Jensen ML, Whishaw IQ (1989) Neurobehavioural deficit due to ischemic brain damage limited to half of the CA1 sector of the hippocampus. J Neurosci 9, 5:16411647.

Auld DS, Kar S, Quirion R (1998) Beta-amyloid peptides as direct cholinergic neuromodulators: a missing link? Trends Neurosci 21, 1:43-49.

Auld DS, Kornecook TJ, Bastianetto S, Quirion R (2002) Alzheimer's disease and the basal forebrain cholinergic system: relations to beta-amyloid peptides, cognition, and treatment strategies. Prog Neurobiol 68, 3:209-245.

Aztiria E, Cataudella T, Spampinato S, Leanza G (2008) Septal grafts restore cognitive abilities and amyloid precursor protein metabolism. Neurobiol Aging Feb 5, Epub ahead of print.

Back T, Hemmen T, Schuler OG (2004) Lesion evolution in cerebral ischemia. J Neurol 251:388-397.

Backhaus J, Born J, Hoesckesfeld R, Fokuhl D, Hohagen F, Junghanns K (2007) Midlife decline in declarative memory consolidation is correlated with a decline in slow wave sleep. Learn Mem 14, 5:336-341.

Ball MJ, Hachinski V, Fox A, Kirshen AJ, Fisman M, Blume W, Kral VA, Fox H (1985) A new definition of Alzheimer's disease: A hippocampal dementia. Lancet Jan.5:14-16.

Bannon AW, Curzon P, Gunther KL, Decker MW (1996) Effects of intraseptal injection of 192-IgG SAPorin in mature and aged Long-Evans rats. Brain Res 718, 1-2:25-36

Barnes LL, Schneider JA, Boyle PA, Bienias JL, Bennett DA (2006) Memory complaints are related to Alzheimer disease pathology in older persons. Neurology 67, 9:1581-1585.

Barnett SA (1963) The Rat: A Study of Behaviour. Principles of ethology and behavioural physiology, displayed mainly in the rat. Methuen and Company Ltd.

Bartus RT (1978) Evidence for a direct cholinergic involvement in the scopolamineinduced amnesia in monkeys: effects of concurrent administration of physostigmine and methylphenidate with scopolamine. Pharmacol Biochem Behav 9, 6:833-836. 
Bartus RT, Dean RL, Goas JA, Lippa AS (1980) Age-related changes in passive avoidance retention: modulation with dietary choline. Science 209, 4453:301-303

Bartus RT, Dean RL 3rd, Beer B, Lippa AS (1982) The cholinergic hypothesis of geriatric memory dysfunction. Science 217, 4558:408-414.

Bartus RT (2000) On neurodegenerative diseases, models, and treatment strategies: lessons learned and lessons forgotten a generation following the cholinergic hypothesis. Exp Neurol 163:495-529.

Baskerville KA, Schweitzer JB, Herron P (1997) Effects of cholinergic depletion on experience-dependent plasticity in the cortex of the rat. Neuroscience, 80, 4: 1159-1169.

Baskerville KA, Kent C, Nicolle MN, Gallagher M, McKinney M (2006) Aging causes partial loss of basal forebrain but no loss of pontine reticular cholinergic neurons. NeuroReport 17:1819-1823.

Bassant MH, Apartis E, Jazat-Poindessous FR, Wiley RG, Lamour YA (1995) Selective immunolesion of the basal forebrain cholinergic neurons: Effects on hippocampal activity during sleep and wakefulness in the rat. Neurodegeneration 4:61-70.

Baxter MG, Bucci DJ, Gorman LK, Wiley RG, Gallagher M (1995) Selective immunotoxic lesions of basal forebrain cholinergic cells: Effects on learning and memory in rats. Behav Neurosci 109, 4:714-722.

Baxter MG, Gallagher M (1996) Intact spatial learning in both young and aged rats following selective removal of hippocampal cholinergic input. Behav Neurosci 110, 3:460-467.

Baxter MG, Holland PC, Gallagher M (1997) Disruption of decrements in conditioned stimulus processing by selective removal of hippocampal cholinergic input. J Neurosci $17,13: 5230-5236$.

Beach TG, Honer WG, Hughes LH (1997) Cholinergic fibre loss associated with diffuse plaques in the non-demented elderly: the preclinical stage of Alzheimer's disease? Acta Neuropathol 93, 2:146-153.

Beaulé C, Amir S (2002) Effect of 192 IgG SAPorin on circadian activity rhythms, expression of P75 neurotrophin receptors, calbindin-D28K, and light-induced Fos in the suprachiasmatic nucleus in rats. Exp Neurol 176, 2:377-389.

Belluardo N, Blum M, Mudo G, Andbjer B, Fuxe K (1998) Acute intermittent nicotine treatment produces regional increases of basic fibroblast growth factor messenger RNA and protein in the tel- and diencephalon of the rat. Neuroscience 83, 3:723-740. 
Belluardo N, Mudo G, Caniglia G, Cheng Q, Blum M, Fuxe K (1999) The nicotinic receptor agonist ABT-594 increases FGF-2 expression in various rat brain regions. NeuroReport 10:3909-3913.

Bellucci A, Luccarini I, Scali C, Prosperi C, Giovannini MG, Pepeu G, Casamenti F (2006) Cholinergic dysfunction, neuronal damage and axonal loss in TgCRND8 mice. Neurobiol Dis 23, 2:260-272.

Ben-Ari Y (1985) Limbic seizure and brain damage produced by kainic acid: mechanisms and relevance to human temporal lobe epilepsy. Neuroscience 14, 2:375403.

Bennett SAL, Pappas BA, Stevens WD, Davidson CM, Fortin T, Chen J (2000) Cleavage of amyloid precursor protein elicited by chronic cerebral hypoperfusion. Neurobiol Aging 21:207-214.

Berger-Sweeney J, Heckers S, Mesulam MM, Wiley RG, Lappi DA, Sharma M (1994) Differential effects on spatial navigation of immunotoxin-induced cholinergic lesions of the medial septal area and nucleus basalis magnocellularis. J Neurosci 14, 7:4507-4519.

Berger-Sweeney J, Stearns NA, Frick KM, Beard B, Baxter MG (2000) Cholinergic basal forebrain is critical for social transmission of food preferences. Hippocampus 10, 6:729738.

Berr C, Hauw JJ, Delaère P, Duyckaerts C, Amouyel P (1994) Apolipoprotein E allele epsilon 4 is linked to increased deposition of the amyloid beta-peptide (A-beta) in cases with or without Alzheimer's disease. Neurosci Lett 178, 2:221-224.

Bina KG, Rusak B, Sembra K (1993) Localization of cholinergic neurons in the forebrain and brainstem that project to the suprachiasmatic nucleus of the hypothalamus in the rat. J Comp Neurol 335:295-307.

Bina KG, Rusak B, Sembra K (1997) Sources of p75-nerve growth factor receptor-like immunoreactivity in the rat suprachiasmatic nucleus. Neuroscience 77, 2:461-472.

Bizon JL, Helm KA, Han JS, Chun HJ, Pucilowska J, Lund PK, Gallagher M (2001) Hypothalamic-pituitary-adrenal axis function and corticosterone receptor expression in behaviourally characterized young and aged Long-Evans rats. Eur J Neurosci 14:17391751.

Bizon JL, Han J-S, Hudon C, Gallagher M (2003) Effects of hippocampal cholinergic deafferentation on learning strategy selection in a visible platform version of the water maze. Hippocampus 13:676-684. 
Bizzarro A, Marra C, Acciarri A, Valenza A, Tiziano FD, Brahe C, Masullo C (2005) Apolipoprotein $\mathrm{E}$ epsilon4 allele differentiates the clinical response to donepezil in Alzheimer's disease. Dement Geriatr Cogn Dis 20, 4:254-261.

Blanchard RJ, Blanchard DC (1969) Crouching as an index of fear. J Comp Physiol Psychol 67:370-375.

Bonnet $\mathrm{MH}$ (1989) Infrequent periodic sleep disruption: effects on sleep, performance and mood. Physiol Behav 45, 5:1040-1055.

Borlongan CV, Shytle RD, Ross SD, Shimizu T, Freeman TB, Cahill DW, Sanberg PR (1995) (-)-nicotine protects against systemic kainic acid-induced excitotoxic effects. Exp Neurol 136, 2:261-265.

Bowen DM, Benton JS, Spillane JA, Smith CC, Allen SJ (1982) Choline acetyltransferase activity and histopathology of frontal neocortex from biopsies of demented patients. J Neurol Sci 57, 2-3:191-202.

Breteler MM, de Groot RR, van Romunde LK, Hofman A (1995) Risk of dementia in patients with Parkinson's disease, epilepsy, and severe head trauma: a register-based follow-up study. Am J Epidemiol 142, 12:1300-1305.

Buzsaki G (1989) Two-stage model of memory trace formation: a role for "noisy" brain states. Neuroscience 31, 3:551-570.

Caldwell HK, Lee HJ, Macbeth AH, Young III WS (2008) Vasopressin: Behavioural roles of an "original" neuropeptide. Prog Neurobiol 84:1-24.

Carlesimo GA, Oscar-Berman M (1992) Memory deficits in Alzheimer's patients: a comprehensive review. Neuropsychol Rev 3, 2:119-169.

Cavazos JE, Das I, Sutula TP (1994) Neuronal loss induced in limbic pathways by kindling: Evidence for induction of hippocampal sclerosis by repeated brief seizures. $\mathrm{J}$ Neurosci 14, 5:3106-3121.

Chang Q, Gold PE (2004) Impaired and spared cholinergic functions in the hippocampus after lesions of the medial septum/vertical limb of the diagonal band with $192 \mathrm{IgG}$ SAPorin. Hippocampus 14, 2:170-179.

Cho C (2001) Chronic 'jet lag' produces temporal lobe atrophy and spatial cognitive deficits. Nat Neurosci 4, 6:567-568.

Cho C, Ennaceur A, Cole JC, Suh CK (2000) Chronic jet lag produces cognitive deficits. J Neurosci 20, RC66:1-5. 
Choi S-A, Kim EH, Lee JY, Nam HS, Kim SH, Kim GW, Lee BI, Heo JH (2007) Preconditioning with chronic cerebral hypoperfusion reduces a focal cerebral ischemic injury and increases apurinic/apyrimidinic endonuclease/redox factor-1 and matrix metalloproteinase-2 expression. Curr Neurovasc Res 4:89-97.

Choi SH, Kim SY, Na HR, Kim BK, Yang DW, Kwon JC, Park MY (2008) Effect of ApoE genotype on response to donepezil in patients with Alzheimer's disease. Dement GeriatrCogn Dis 25, 5:445-450.

Connelly PJ, Prentice NP, Fowler KG (2005) Predicting the outcome of cholinesterase inhibitor treatment in Alzheimer's disease. J Neurol Neurosurg Psychiatry 76:320-324.

Conner JM, Culberson A, Packowski C, Chiba AA, Tuszynski MH (2003) Lesions of the basal forebrain system impair task acquisition and abolish cortical plasticity associated with motor skill learning. Neuron 38:819-829.

Conrad C, Galea LAM, Kuroda Y, McEwen BS (1996) Chronic stress impairs rat spatial memory on the $\mathrm{Y}$ maze and this effect is blocked by Tianeptine pretreatment. Behav Neurosci 110, 6:1321-1334.

Cooper-Kuhn CM, Winkler J, Kuhn HG (2004) Decreased neurogenesis after cholinergic forebrain lesion in the adult rat. J Neurosci Res 77:155-165.

Corder EH, Saunders AM, Strittmatter WJ, Schmechel DE, Gaskell PC, Small GW, Roses AD, Haines JL, Pericak-Vance MA (1993) Gene dose of apolipoprotein E type 4 allele and the risk of Alzheimer's disease in late onset families. Science 261, 5123:921923.

Connelly PJ, Prentice NP, Fowler KG (2005) Predicting the outcome of cholinesterase inhibitor treatment in Alzheimer's disease. J Neurol Neurosurg Psychiatry 76, 3:320-324.

Courtney C, Farrell D, Gray R, Hills R, Lynch L, Sellwood E, Edwards S, Hardyman W, Raftery J, Crome P, Lendon C, Shaw H, Bentham P, AD2000 Collaborative Group (2004) Long-term donepezil treatment in 565 patients with Alzheimer's disease (AD2000): randomised double-blind trial. Lancet 363, 9427:2105-2115.

Costa G, Abin-Carriquiry JA, Dajas F (2001) Nicotine prevents striatal dopamine loss produced by 6 -hydroxydopamine lesion in the substantia nigra. Brain Res 888 , 2:336342.

Craig LA, McDonald RJ (2005) Multiple combinations of co-factors produce variants of age-related cognitive decline. Society for Neuroscience Abstract, Washington, D.C.

Craig LA, McDonald RJ (2008) Chronic circadian disruption impairs hippocampal memory in the rat. Brain Res Bull 76, 1-2:141-151. 
Craig LA, Hong NS, Kopp J, McDonald RJ (2008) Emergence of spatial impairment in rats following specific cholinergic depletion of the medial septum combined with chronic stress. Eur J Neurosci 27:2262-2271.

Craig LA, Hong NS, Kopp J, McDonald RJ (2008) Reduced cholinergic status in hippocampus produces spatial memory deficits when combined with kainic acid induced seizures. Hippocampus July 23 epub ahead of print.

Crespo-Biel N, Canudas AM, Camins A, Pallàs M (2006) Kainate induces AKT, ERK and cdk5/GSK3beta pathway deregulation, phosphorylates tau protein in mouse hippocampus. Neurochem Int 50, 2:435-442.

Csernansky JG, Dong H, Fagan AM, Wang L, Xiong C, Holtzman DM, Morris JC (2006) Plasma control and progression of dementia in DAT subjects. Am J Psychiatry 163, 12:2164-2169.

Dahlqvist P, Ronnback A, Bergstrom S-A, Soderstrom I, Olsson T (2004) Environmental enrichment reverses learning impairment in the Morris water maze after focal cerebral ischemia in rats. Eur J Neurosci 19:2288-2298.

Dajas-Bailador FA, Lima PA, Wonnacott S (2000) The $\alpha 7$ nicotinic acetylcholine receptor subtype mediates nicotinic protection against NMDA excitotoxicity in primary hippocampal cultures through a $\mathrm{Ca} 2+$ dependent mechanism. Neuropharmacology 39:2799-2807.

Damiano BP, Connor JD (1984) Hippocampal mediation of shaking behaviour induced by electrical stimulation of the perforant path in the rat. Brain Res 308:383-386.

Davies P, Maloney AJ (1976) Selective loss of central cholinergic neurons in Alzheimer's disease. Lancet 8000:1403.

Davis KL, Davis BM, Greenwald BS, Mohs RC, Mathe AA, Johns CA, Horvath TB (1986) Cortisol and Alzheimer's disease, I: Basal studies. Am J Psychiatry 143, 3:300305.

De Jong GI, Farkas E, Stienstra CM, Plass JRM, Keijser JN, de la Torre JC, Luiten PGM. (1999) Cerebral hypoperfusion yields capillary damage in the hippocampal CA1 area that correlates with spatial memory impairment. Neuroscience 91, 1:203-210.

de la Torre JC, Fortin T, Park GAS, Butler KS, Kozlowski P, Pappas BA, Socarraz H, Saunders JK, Richard MT (1992) Chronic cerebrovascular insufficiency induces dementia-like deficits in aged rats. Brain Res 582:186-195.

de la Torre JC (2002) Alzheimer disease as a vascular disorder: nosological evidence. Stroke 33, 4:1152-1162 
Del Vecchio RA, Gold LH, Novick SJ, Wong G, Hyde LA (2004) Increased seizure threshold and severity in young transgenic CRND8 mice. Neurosci Lett 367:164-167.

Devan BD, McDonald RJ, White NM (1999) Effects of medial and lateral caudateputamen lesions on place- and cue-guided behaviours in the water maze: relation to thigmotaxis. Behav Brain Res 100:5-14.

Devan BD, Goad EH, Petri HL, Antoniadis EA, Hong NS, Ko CH, Leblanc L, Lebovic SS, Lo Q, Ralph MR, McDonald RJ (2001) Circadian phase-shifted rats show normal acquisition but impaired long-term retention of place information in the water task. Neurobiology of Learn Mem 75:51-62.

Doody RS, Dunn JK, Clark CM, Farlow M, Foster NL, Liao T, Gonzales N, Lai E, Massman $P$ (2001) Chronic donepezil treatment is associated with slowed cognitive decline in Alzheimer's disease. Dement Geriatr Cogn Dis 12, 4:295-300.

Drachman DA, Leavitt J (1974) Human memory and the cholinergic system. A relationship to aging? Arch Neurol 30, 2:113-121.

Drachman DA (1977) Memory and cognitive function in man: does the cholinergic system have a specific role? Neurology 27, 8:783-790.

Driscoll I, Howard SR, Stone JC, Monfils MH, Tomanek B, Brooks WM, Sutherland RJ (2006) The aging hippocampus: a multi-level analysis in the rat. Neuroscience 139, 4:1173-1185.

Driscoll I, Hong NS, Craig LA, Sutherland RJ, McDonald RJ (2007) Enhanced cell death and learning deficits after a mini-stroke in aged hippocampus. Neurobiol Aging doi:10.1016/j.neurobiolaging.2007.04.025.

Dunnett SB, Whishaw IQ, Jones GH, Bunch ST (1987) Behavioural, biochemical and histochemical effects of different neurotoxic amino acids injected into nucleus basalis magnocellularis of rats. Neuroscience 20:653-669.

Durukan A, Tatlisumak T (2007) Ischemic stroke: Overview of major experimental rodent models, pathophysiology, and therapy of focal cerebral ischemia. Pharmacol Biochem Behav 87:179-197.

Eldridge LL, Masterman D, Knowlton BJ (2002) Intact implicit habit learning in Alzheimer's disease. Behav Neurosci 116, 4:722-726.

Endo Y, Shinohara K, Fueta Y, Irie M (2001) Influences of cholinergic neurotoxin ethylcholine aziridinium ion on circadian rhythms in rats. Neurosci Res 41, 4:385-390. 
Erhardt C, Galani R, Jeltsch H, Cassel JC, Klosen P, Menet JS, Pévet P, Challet E (2004) Modulation of photic resetting in rats by lesions of projections to the suprachiasmatic nuclei expressing p75 neurotrophin receptor. Eur J Neurosci 19, 7:1773-1788.

Faherty CJ, Kerley D, Smeyne RJ (2003) A Golgi-Cox morphological analysis of neuronal changes induced by environmental enrichment. Dev Brain Res 14:55-61.

Fanselow MS, LeDoux JE (1999) Why I think plasticity underlying Pavlovian fear conditioning occurs in the basolateral amygdala. Neuron 23:229-232.

Ferbinteanu J, Ray C, McDonald RJ (2003) Both dorsal and ventral hippocampus contribute to spatial learning in Long-Evans rats. Neurosci Lett 345:131-135.

Farkas E, Luiten PGM (2001) Cerebral microvascular pathology in aging and Alzheimer's disease. Prog Neurobiol 64:575-611.

Fekete M, van Ree JM, Niesink RJM, de Wied D (1985) Disrupting circadian rhythms in rats induces retrograde amnesia. Physiol Behav 34:883-887.

Feng Z, Chang Y, Cheng Y, Zhang BL, Qu ZW, Qin C, Zhang JT (2004) Melatonin alleviates behavioural deficits associated with apoptosis and cholinergic system dysfunction in the APP 695 transgenic mouse model of Alzheimer's disease. J Pineal Res 37, 2:129-136.

Ferencz I, Kokaia M, Keep M, Elmer E, Metsis M, Kokaia Z, Lindvall O (1997) Effects of cholinergic denervation on seizure development and neurotrophin messenger RNA regulation in rapid hippocampal kindling. Neuroscience 80, 2:389-399.

Ferencz I, Leanza G, Nanobashvili A, Kokaia Z, Kokaia M, Lindvall O (2001) Septal cholinergic neurons suppress seizure development in hippocampal kindling in rats: comparison with noradrenergic neurons. Neuroscience 102, 4:819-832.

Ferguson SA, Kennaway DJ, Moyer RW (1999) Nicotine phase shifts the 6sulphatoxymelatonin rhythm and induces c-Fos in the SCN of rats. Brain Res Bull 48, 5:527-538.

Ferrer I, Krupinski J, Goutan E, Martí E, Ambrosio S, Arenas E (2001) Brain-derived neurotrophic factor reduces cortical cell death by ischemia after middle cerebral artery occlusion in the rat. Acta Neuropathol 101, 3229-238.

Fischer W, Chen KS, Gage FH, Bjorklund A (1991) Progressive decline in spatial learning and integrity of forebrain cholinergic neurons in rats during aging. Neurobiol Aging 13:9-23.

Fisher A (2000) Therapeutic strategies in Alzheimer's disease: M1 muscarinic agonists. Jap J Pharmacol 84, 2:101-212. 
Fleischman DA, Wilson RS, Gabrieli JDE, Schneider JA, Bienias JL, Bennett DA (2005) Implicit memory and Alzheimer's disease neuropathology. Brain 128:2006-2015.

Fleischman DA, Gabrieli J (1999) Long-term memory in Alzheimer's disease. Curr Op Neurobiol 9:240-244.

Frankland PW, Cestari V, Filipkowski RK, McDonald RJ, Silva AJ (1998) The dorsal hippocampus is essential for context discriminations, but not for context recognition. Behav Neurosci112:863-874.

French SJ, Humby T, Horner CH, Sofroniew MV, Rattray M (1999) Hippocampal neurotrophin and trk receptor mRNA levels are altered by local administration of nicotine, carbachol and pilocarpine. Mol Brain Res 67:124-136.

Frick KM, Kim JJ, Baxter MG (2004) Effects of complete immunotoxin lesions of cholinergic basal forebrain on fear conditioning and spatial learning. Hippocampus 14:244-254.

Frielingsdiorf H, Thal LJ, Pizzo DP (2006) The septohippocampal system and spatial working memory in the Morris water maze. Behavl Brain Res 168, 1:609-613.

Fujikawa DG (2005) Prolonged seizures and cellular injury: Understanding the connection. Epilepsy Behav 7:S3-S11.

Fujiki M, Kobayashi H, Uchida S, Inoue R, Ishii K (2005) Neuroprotective effect of donepezil, a nicotinic acetylcholine-receptor activator, on cerebral infarction in rats. Brain Res 1043:236-241.

Fujii S, Sumikawa K (2001) Acute and chronic nicotine exposure reverse age-related declines in the induction of long-term potentiation in the rat hippocampus. Brain Res 894:347-353.

Fuxe K, Kurosawa N, Cintra A, Hallstrom A, Goiny M, Rosen L, Agnati LF, Ungerstedt $U$ (1992) Involvement of local ischemia in endothelin- 1 induced lesions of the neostriatum of the anaesthetized rat. Exp Brain Res 88:131-139.

Gabriel EM, Inglefield JR, Chadwick LE, Schawartz-Bloom RD (1998) Ischemic injury and extracellular amino acid accumulation in hippocampal area CA1 are not dependent upon an intact septo-hippocampal pathway. Brain Res 785:279-286.

Gadek-Michalska A, Bugajski J (2003) Repeated handling, restraint, or chronic crowding impair the hypothalamic-pituitary-adrenocortical response to acute restraint stress. J Physiol Pharmacol 54, 3:449-459. 
Gallagher M, Colombo PJ (1995) Aging: the cholinergic hypothesis of cognitive decline. Curr Op Neurobiol 5:161-168.

Games D, Buttini M, Kobayashi D, Schenk D, Seubert P (2006) Mice as models: transgenic approaches and Alzheimer's disease. J Alzheimers Dis 9, 3, Suppl:133-149.

Gayoso MJ, Primo C, Al-Majdalawi A, Fernandez JM, Garrosa M, Iniguez C (1994) Brain lesions and water-maze learning deficits after systemic administration of kainic acid to adult rats. Brain Res 653:92-100.

Geddes JW, Cotman CW. (1986) Plasticity in hippocampal excitatory amino acid receptors in Alzheimer's disease. Neurosci Res 3, 6:672-678.

Geula C, Nagykery N, Nicholas A, Wu CK (2008) Cholinergic neuronal and axonal abnormalities are present early in aging and in Alzheimer disease. J Neuropathol Exp Neurol 67, 4:309-318.

Gilbert TH, McNamara RK, Corcoran ME (1996) Kindling of hippocampal field CA1 impairs spatial learning and retention in the Morris water maze. Behav Brain Res 82:5766.

Gilbert TH, Hannesson DK, Corcoran ME (2000) Hippocampal kindled seizures impair spatial cognition in the Morris water maze. Epilepsy Res 38:115-125.

Gilmour G, Iversen SD, O'Neill MF, Bannerman DM (2004) The effects of intracortical endothelin-1 injections on skilled forelimb use: implications for modeling recovery of function after stroke. Behav Brain Res 150:171-183.

Glavin GB, Pare WP, Sandbak T, Bakke H-K, Murison R (1994) Restraint stress in biomedical research: An update. Neurosci Biobehav Rev 18, 2:223-249.

Glenner GG, Wong CW (1984) Alzheimer's disease: initial report of the purification and characterization of a novel cerebrovascular amyloid protein. Biochem Biophysiol Res Comm 120, 3:885-890.

Gobbo OL, O'Mara SM (2004) Post-treatment, but not pre-treatment, with the selective cyclooxygenase- 2 inhibitor celecoxib markedly enhances functional recovery from kainic acid-induced neurodegeneration. Neuroscience 125:317-327.

Gobbo OL, O'Mara SM (2005) Exercise but not environmental enrichment, improves learning after kainic acid-induced hippocampal neurodegeneration in association with an increase in brain-derived neurotrophin factor. Behav Brain Res 159:21-26.

Goddard GV, McIntyre DC, Leech CK (1969) A permanent change in brain function resulting from daily electrical stimulation. Exp Neurol 25, 3:295-330. 
Goedert M, Wischik CM, Crowther RA, Walker JE, Klug A (1988) Cloning and sequencing of the cDNA encoding a core protein of the paired helical filament of Alzheimer disease: identification as the microtubule-associated protein tau.

PNAS U S A 85, 11:4051-4055.

Goedert M, Klug A, Crowther RA (2006) Tau protein, the paired helical filament and Alzheimer's disease. J Alzheimer's Dis 9, 3, Supplement:195-207.

Goichot B, Weibel L, Chapotot F, Gronfier C, Piquard F, Brandenberger G (1998) Effect of the shift of the sleep-wake cycle on three robust endocrine markers of the circadian clock. Am J Physiol, 275, 2, 1:E243-8.

Golden GT, Smith GG, Ferraro TN, Reyes PF, Kulp JK, Fariello RG (1991) Strain difference in convulsive response to the excitotoxin kainic acid. Neuroreport 2, 3:141144.

Golden GT, Smith GG, Ferraro TN, Reyes PF (1995) Rat strain and age differences in kainic acid induced seizures. Epilepsy Res 20, 2:151-159.

Gonzalez CLR, Gharbawie OA, Kolb B (2006) Chronic low-dose administration of nicotine facilitates recovery and synaptic change after focal ischemia in rats. Neuropharmacology, 50: 777-787.

Gouirand AM, Matuszewich L (2005) The effects of chronic unpredictable stress on male rats in the water maze. Physiol Behav 86:21-31.

Grau-Olivares M, Bartres-Faz D, Arboix A, Soliva JC, Rovira M, Targa C, Junque C (2007) Mild cognitive impairment after lacunar infarction: voxel-based morphometry and neuropsychological assessment. Cerebrovasc Dis 23, 5-6:353-361.

Green KN, Billings LM, Roozendaal S, McGaugh JL, LaFerla FM (2006) Glucocorticoids increase amyloid- $\beta$ and tau pathology in a mouse model of Alzheimer's disease. J Neurosci 26, 35:9047-9056.

Gregus A, Wintink AJ, Davis AC, Kalynchuk LE (2005) Effect of repeated corticosterone injections and restraint stress on anxiety and depression-like behaviour in male rats. Behav Brain Res 156:105-114.

Guillozet $A L$, Weintraub S, Mash DC, Mesulam MM (2003) Neurofibrillary tangles, amyloid, and memory in aging and mild cognitive impairment. Arch Neurol 60, 5:729736.

$\mathrm{Gu} \mathrm{Z}, \mathrm{Yu}$ J, Perez-Polo JR (1998) Responses in the aged rat brain after total immunolesion. J Neurosci Res 54, 1:7-16. 
Guo Q, Fu W, Sopher BL, Miller MW, Ware CB, Martin GM, Mattson MP:(1999) Increased vulnerability of hippocampal neurons to excitotoxic necrosis in presenilin-1 mutant knock-in mice. Nat Med 5, 1:101-106.

Han F, Raie AA, Shioda N, Qin Z-H, Fukunaga K (2008) Accumulation of beta-amyloid in the brain microvessels accompanies increased hyperphosphorylated tau proteins following microsphere embolism in aged rats, Neuroscience, doi:

10.1016/j.neuroscience.2008.02.044

Han JS, Bizon JL, Chun HJ, Maus CE, Gallagher M (2002) Decreased glucocorticoid receptor mRNA and dysfunction of HPA axis in rats after removal of the cholinergic innervation to hippocampus. Eur J Neurosci 16:1399-1404.

Harati H, Barbelivien A, Cosquer B, Majchrzak M, Cassel JC (2008) Selective cholinergic lesions in the rat nucleus basalis magnocellularis with limited damage in the medial septum specifically alter attention performance in the five-choice serial reaction time task. Neuroscience 153, 1:72-83.

Hardy J, Allsop D (1991) Amyloid deposition as the central event in the aetiology of Alzheimer's disease. Trends in Pharmacological Science 12, 10:383-388.

Harmon KM, Wellman CL (2003) Differential effects of cholinergic lesions on dendritic spines in frontal cortex of young adult and aging rats. Brain Res 992:60-68.

Hatfield CF, Herbert J, van Someren EJW, Hodges JR, Hastings MH (2004) Disrupted daily activity/rest sessions in relation to daily cortisol rhythms of home-dwelling patients with early Alzheimer's dementia. Brain 127:1061-1074.

Hauser WA, Morris ML, Heston LL, Anderson VE (1986) Seizures and myoclonus in patients with Alzheimer's disease. Neurology 36:1226-1230.

Heckers S, Ohtake T, Wiley RG, Lappi DA, Geula C, Mesulam MM (1994) Complete and selective cholinergic denervation of rat neocortex and hippocampus but not amygdala by an immunotoxin against the p75 NGF receptor. J Neurosci 14:1271-1289.

Heine VM, Maslam S, Zareno J, Joels M, Lucassen PJ (2004) Suppressed proliferation and apoptotic changes in the rat dentate gyrus after acutẽ and chronic stress are reversible. Eur J Neurosci 19:131-144.

Hellier JL, Patrylo PR, Buckmaster PS, Dudek FE (1998) Recurrent spontaneous motor seizures after repeated low-dose systemic treatment with kainate: assessment of a rat model of temporal lobe epilepsy. Epilepsy Res 1:73-84.

Hellier JL, Patrylo PR, Dou P, Nett M, Rose GM, Dudek FE (1999)Assessment of inhibition and epileptiform activity in the septal dentate gyrus of freely behaving rats during the first week after kainate treatment. J Neurosci 19, 22:10053-10064. 
Helm KA, Ziegler DR, Gallagher M (2004) Habituation to stress and dexamethasone suppression in rats with selective basal forebrain cholinergic lesions. Hippocampus 14:628-635.

Hesdorffer DC, Hauser WA, Annegers JF, Kokmen E, Rocca WA (1996) Dementia and adult-onset unprovoked seizures. Neurology 46, 3:727-730.

Hodges JR, Patterson K (1994) Is semantic memory consistently impaired early in the course of Alzheimer's disease? Neuroanatomical and diagnostic implications.

Neuropsychologia 33, 4:441-459.

Hofman MA, Swaab DF (1994) Alterations in circadian rhythmicity of the vasopressinproducing neurons of the human suprachiasmatic nucleus (SCN) with aging. Brain Res 651:134-142.

Honkaniemi J, Massa SM, Breckinridge M, Sharp FR (1996) Global ischemia induces apoptosis-associated genes in hippocampus. Mol Brain Res 42:79-88.

Hurd MW, Ralph MR (1998) The significance of circadian organization for longevity in the rat. J Bio Rhythms 13, 5:430-436.

Hyman BT, Van Hoesen GW, Damasio AR, Barnes CL (1984) Alzheimer's disease: cellspecific pathology isolates the hippocampal formation. Science 225, 4667:1168-1170.

Ickes BR, Pham TM, Sanders LA, Albeck DS, Mohammed AH, Granholm AC (2000) Long-term environmental enrichment leads to regional increases in neurotrophin levels in rat brain. Exp Neurol 164, 1:45-52.

Janik D, Godfrey M, Mrosovsky N (1998) Phase angle changes of photically entrained circadian rhythms following a single non-photic stimulus. Physiol Behav 55:103-107.

Janis LS, Glasier MM, Fulop Z, Stein DG (1998) Intraseptal injections of 192-IgG saporin produce deficits for strategy selection in spatial-memory tasks. Behav Brain Res 90:23-34.

Jaspers RMA, Block F, Heim C, Sontag KH (1990) Spatial learning is affected by transient occlusion of common carotid arteries (2VO): comparison of behavioural and histopathological changes after '2VO' and 'four-vessel-occlusion' in rats. Neurosci Lett 117:149-153.

Jeong YH, Park CH, Yoo J, Shin KY, Ahn SM, Kim HS, Lee SH, Emson PC, Suh YH (2006) Chronic stress accelerates learning and memory impairments and increases amyloid deposition in APPV7171CT100 transgenic mice, an Alzheimer's disease model. FASEB 20, 6:729-741. 
Ji D, Lape R, Dani JA (2001) Timing and location of nicotinic activity enhances or depresses hippocampal synaptic plasticity. Neuron 31:131-141.

Jin K, LaFevre-Bernt M, Sun Y, Chen S, Gafni J, Crippen D, Logvinova A, Ross CA, Greenberg DA, Ellerby LM (2005) FGF-2 promotes neurogenesis and neuroprotection and prolongs survival in a transgenic mouse model of Huntington's disease. PNAS 102, 50:18189-18194.

Johnson SA, Pasinetti GM, May PC, Ponte PA, Cordell B, Finch CE (1988) Selective reduction of mRNA for the ?-amyloid precursor protein that lacks a Kunitz-type protease inhibitor motif in cortex from Alzheimer brains. Exp Neurol 102:264-268.

Jonasson Z, Cahill JF, Tobey RE, Baxter MG (2004) Sexually dimorphic effects of hippocampal cholinergic deafferentation in rats. Eur J Neurosci 20, 11:3041-3053.

Jouvenceau A, Billard JM, Lamour Y, Dutar P(1996) Persistence of CA1 hippocampal LTP after selective cholinergic denervation. Neuroreport 7, 4:948-952.

Jouvenceau A, Billard JM, Lamour Y, Dutar P (1997) Potentiation of glutamatergic EPSP's in rat CA1 hippocampal neurons after selective cholinergic denervation by 192 IgG SAPorin. Synapse 26:292-300.

Kapas L, Obal Jr F, Book AA, Schweitzer JB, Wiley RG, Krueger JM (1996) The effects of immunolesions of nerve growth factor-receptive neurons by 192 IgG SAPorin on sleep. Brain Res 712:53-59.

Kar S, Slowikowski SP, Westaway D, Mount HT (2004) Interactions between betaamyloid and central cholinergic neurons: implications for Alzheimer's disease. J Psychiatry Neurosci 29, 6:427-441.

Karczmar AG, Chauhan N, Christopoulos A, Lindstrom J, Siegel GJ (2007) Exploring the Vertebrate Central Cholinergic Nervous System. Springer Science + Business Media, LLC. New York, NY.

Karhunen H, Nissinen J, Sivenius J, Jolkkonen J, Pitkanen A (2006) A long-term videoEEG and behavioural follow-up after endothelin-1 induced middle cerebral artery occlusion in rats. Epilepsy Res 72:25-38.

Keefe DL, Earnest DJ, Nelson D, Takahashi JS, Turek FW (1987) A cholinergic antagonist, mecamylamine, blocks the phase-shifting effects of light on the circadian rhythm of locomotor activity in the golden hamster. Brain Res 403, 2:308-312.

Kenny PJ, File SE, Rattray M (2000) Acute nicotine decreases, and chronic nicotine increases the expression of brain-derived neurotrophic factor mRNA in rat hippocampus. Brain Res Mol Brain Res 85, 1-2:234-238. 
Kilgard MP, Merzenich MM (1998) Cortical map reorganization enabled by nucleus basalis activity. Science 279, 5357:1714-1718.

Kim SH, Kim EH, Lee BI, Heo JH (2008) Chronic cerebral hypoperfusion protects against acute focal ischemia, improves motor function, and results in vascular remodeling. Curr Neurovas Res 5:28-36.

Kim JJ, Fanselow MS (1992) Modality-specific retrograde amnesia of fear. Science 256:675-677.

Kirino T, Sano K (1984) Selective vulnerability in the gerbil hippocampus following transient ischemia. Acta Neuropathol 62:201-208.

Kivipelto M, Rovio S, Ngandu T, Kareholt I, Eskelinen M, Winblad B, Hachinski V, Cedazo-Minguez A, Soininene H, Tuomilehto J, Nissinene A (2008) Apolipoprotein E $\varepsilon 4$ magnifies lifestyle risks for dementia: a population based study. J Cell Mol Med "Postprint": 10.1111/j.1582-4934.2008.00296.x.

Kiyota Y, Miyamoto M, Nagaoka A (1991) Relationship between brain damage and memory impairment in rats exposed to transient forebrain ischemia. Brain Res 538:295302.

Knipper M, da Penha Berzaghi M, Blochl A, Breer H, Thoenen H, Lindholm D (1994) Positive feedback between acetylcholine and the neurotrophins nerve growth factor and brain-derived neurotrophic factor in the rat hippocampus. Eur J Neurosci 6:668-671.

Knutsson A (2004) Methodological aspects of shift-work research. Chronobiol Int 21, 6:1037-1047.

Kokaia M, Ferencz I, Leanza G, Elmer E, Metsis M, Kokaia Z, Wiley RG, Lindvall O (1996) Immunolesioning of basal forebrain cholinergic neurons facilitates hippocampal kindling and perturbs neurotrophin messenger RNA regulation. Neuroscience 70, 2:313327.

Kolb B, Forgie M, Gibb R, Gorny G, Rowntree S. (1998) Age, experience and the changing brain. Neurosci Biobehav Rev 22, 2:143-159.

Kolb B, Gibb R (1991) Environmental enrichment and cortical injury: behavioural and anatomical consequences of frontal cortex lesions. Cereb Cortex 1, 2:189-198.

Larson EB, Wang L, Bowen JD, McCormick WC, Teri L, Crane P, Kukull W (2006) Exercise is associated with reduced risk for incident dementia among persons 65 years of age and older. Ann Intern Med 144, 2:73-81. 
Leanza G (1998) Chronic elevation of amyloid precursor protein expression in the neocortex and hippocampus of rats with selective cholinergic lesions. Neurosci Lett 257, 1:53-56.

Lehmann O, Grottick AJ, Cassel JC, Higgins GA (2003) A double dissociation between serial reaction time and radial maze performance in rats subjected to $192 \mathrm{IgG}$ SAPorin lesions of the nucleus basalis and/or the septal region. Eur J Neurosci 18, 3:651-666.

Lemstra AW, Richard E, van Gool WA (2007) Cholinesterase inhibitors in dementia: yes, no, or maybe? Age Ageing 36, 6:625-627.

Leung LS, Boon KA, Kaibara T, Innis NK (1990) Radial maze performance following hippocampal kindling. Behav Brain Res 40:119-129.

Leung LS, Shen B (1991) Hippocampal CA1 evoked response and radial 8-arm maze performance after hippocampal kindling. Brain Res 555:353-357.

Lin L, Georgievska B, Mattsson A, Isacson O (1999) Cognitive changes and modified processing of amyloid precursor protein in the cortical and hippocampal system after cholinergic synapse loss and muscarinic receptor activation. PNAS 96, 21:12108-12113.

Liu J, Jin D-Z, Xiao L, Zhu X-Z (2006) Paeoniflorin attenuates chronic cerebral hypoperfusion-induced learning dysfunction and brain damage in rats. Brain Res 1089:162-170.

Liu RY, Zhou JN, van Heerikhuize J, Hofman MA, Swaab DF (1999) Decerased melatonin levels in post-mortem cerebrospinal fluid in relation to aging, Alzheimer's disease, and apolipoprotein E-epsilon4/4 genotype. J Clin Endocrinol Metabol 84, 1:323327.

Lothman EW, Collins RC (1981) Kainic acid induced limbic seizures: metabolic, behavioural, electroencephalographic and neuropathological correlates. Brain Res 218, 12:299-318.

Luchsinger JA, Reitz C, Honig LS, Tang MX, Shea S, Mayeux R (2005) Aggregation of vascular risk factors and risk of incident Alzheimer disease. Neurology 65, 4:545-551.

Lue LF, Kuo YM, Roher AE, Brachova L, Shen Y, Sue L, Beach T, Kurth JH, Rydel RE, Rogers J (1999) Soluble amyloid beta peptide concentration as a predictor of synaptic change in Alzheimer's disease. Am J Pathol155, 3:853-862.

Luine V, Villegas M, Martinez C, McEwen BS (1994) Repeated stress causes reversible impairments of spatial memory performance. Brain Res 639:167-170.

Luine V, Martinez C, Villegas M, Margarinos AM, McEwen BS (1996) Restraint stress reversibly enhances spatial memory performance. Physiol Behav 59, 1:27-32. 
Luo CX, Jiang J, Zhou QG, Zhu XJ, Wang W, Zhang ZJ, Han X, Zhu DY (2007) Voluntary exercise-induced neurogenesis in the postischemic dentate gyrus is associated with spatial memory recovery from stroke. J Neurosci Res 85:1637-1646.

Lupien SJ, de Leon M, de Santi S, Convit A, Tarshish C, Nair NPV, Thakur M, McEwen BS, Hauger RL, Meaney MJ (1998) Cortisol levels during human aging predict hippocampal atrophy and memory deficits. Nat Neurosci 1, 1:69-73.

Madeira MD, Pereira PA, Silva SM, Cadete-Leite A, Paula-Barbosa MM (2004) Basal forebrain neurons modulate the synthesis and expression of neuropeptides in the rat suprachiasmatic nucleus. Neuroscience 125:889-901.

Magarinos AM, McEwen BS (1995) Stress-induced atrophy of apical dendrites of hippocampal CA3c neurons: Comparison of stressors. Neuroscience 69:83-88.

Maggio R, Riva M, Vaglini F, Fornai F, Molteni R, Armogida M, Racagni G, Corsini GU (1998) Nicotine prevents experimental parkinsonism in rodents and induces striatal increase of neurotrophic factors. J Neurochem 71, 6:2439-2446.

Malberg JE, Eisch AJ, Nestler EJ, Duman RS (2000). Chronic antidepressant treatment increases neurogenesis in adult rat hippocampus. J Neurosci 20, 24:9104-9110.

Marin MT, Cruz FC, Planeta CS (2007) Chronic restraint or variable stresses differently affect the behaviour, corticosterone secretion and body weight in rats. Physiol Behav 90:29-35.

Markgraf CG, Green EJ, Hurwitz BE, Morikawa E, Dietrich WD, McCabe PM, Ginsberg MD, Schneiderman N (1992) Sensorimotor and cognitive consequences of middle cerebral artery occlusion in rats. Brain Res 575:238-246.

Marques CM, Caboclo LO, da Silva TI, Noffs MH, Carrete H Jr, Lin K, Lin J, Sakamoto AC, Yacubian EM (2007) Cognitive decline in temporal lobe epilepsy due to unilateral hippocampal sclerosis. Epilepsy Behav10, 3:477-485.

Marston HM, Everitt BJ, Robbins TW (1993) Comparative effects of excitotoxic lesions of the hippocampus and septum/diagonal band on conditional visual discrimination and spatial learning. Neuropsychologia 31, 10:1099-1118.

Marston HM, West HL, Wilkinson LS, Everitt BJ, Robbins TW (1994) Effects of excitoxic lesions of the septum and vertical limb of the nucleus of the diagonal band of Broca on conditional visual discrimination: relationship between performance and choline acetyltransferase activity in the cingulated cortex. J Neurosci 14:2009-2019.

Martin SJ, Grimwood PD, Morris RGM (2000) Synaptic plasticity and memory: An evaluation of the hypothesis. Ann Rev Neurosci 23:649-711. 
Martínez-Rodríguez R, Toledano A, Alvarez MI, Turégano L, Colman O, Rosés P, Gómez de Segura I, De Miguel E (2003) Chronic nicotine administration increases NGFlike immunoreactivity in frontoparietal cerebral cortex. J Neurosci Res 73, 5:708-716.

Masters CL, Simms G, Weinman NA, Multhaup G, McDonald BL, Beyreuther K (1985) Amyloid plaque core protein in Alzheimer disease and Down syndrome. PNAS USA 82, 12:4245-4249.

Matsubara E, Bryant-Thomas T, Pacheco Quinto J, Hen Poeggeler B, Herbert D, CruzSanchez F, Chyan YJ, Smith G, Shoji M, Abe K, Leone A, Grundke-Ikbal I, Wilson GL, Williams C, Refolo LM, Pappolla MA, Chain DG, Neria E (2003) Melatonin increases survival and inhibits oxidative amyloid pathology in a transgenic model of Alzheimer's disease. J Neurochem 85, 5:1101-1108.

Matsuyama S, Matsumoto A, Enomoto T, Nishizaki T (2000) Activation of nicotinic acetylcholine receptors induces long-term potentiation in vivo in the intact mouse dentate gyrus. Eur J Neurosci 12:3741-3747.

McArthur JJ, Gillete MM, Prosser RA (1991) Melatonin directly resets the rat suprachiasmatic circadian clock in vitro. Brain Res 565:158-161.

McDonald RJ, White NM (1993) A triple dissociation of memory systems: hippocampus, amygdala, and dorsal striatum. Behav Neurosci 107:3-22.

McDonald RJ, White NM (1994) Parallel information processing in the water maze: evidence for independent memory systems involving dorsal striatum and hippocampus. Behav Neural Biol 61, 3:260-270.

McDonald RJ (2002) Multiple combinations of co-factors produce variants of age-related cognitive decline: A theory. Can J Exp Psychol 56, 3:221-339.

McDonald RJ, Hong NS, Ray C, Ralph MR (2002) No time of day modulation or time stamp on multiple memory tasks in rats. Learn Motiv 33:230-252.

McDonald RJ, Devan BD, Hong NS (2004) Multiple memory systems: the power of interactions. Neurobiol Learn Mem 82, 3:333-346.

McDonald RJ, Hong NS, Craig LA, Holahan MR, Louis M, Muller RU (2005) NMDAreceptor blockade by CPP impairs post-training consolidation of a rapidly acquired spatial representation in rat hippocampus. Eur J Neurosci 22:1201-1213.

McDonald RJ, Craig LA, Hong NS (2008) Enhanced cell death in hippocampus and emergence of cognitive impairments following a localized mini-stroke in hippocampus if preceded by a previous episode of acute stress. Eur J Neurosci 27, 8:2197-2209. 
McEwen BS, Sapolsky RM (1995) Stress and cognitive function. Curr Op Nieurobiol 5, 2:205-216.

McGaughey J, Everitt BJ, Robbins TW, Sarter M (2000) The role of cortical cholinergic afferent projections in cognition: impact of new selective immunotoxins. Behav Brain Res 115:251-263.

McGeer PL, McGeer EG, Suzuki J, Dolman CE, Nagai T (1984) Aging, Alzheimer's disease, and the cholinergic system of the basal forebrain. Neurology 34:741-746.

McGehee DS (2002) Nicotinic receptors and hippocampal synaptic plasticity... it's all in the timing. Trends Neurosci 25, 4:171-172.

McLaughlin KJ, Gomez JL, Baran SE, Conrad CD (2007)The effects of chronic stress on hippocampal morphology and function: an evaluation of chronic restraint paradigms. Brain Res 1161:56-64.

McLay RN, Freeman SM, Zadina JE (1998) Chronic corticosterone impairs memory performance in the Barnes maze. Physiol Behav 63, 5:933-937.

Mesulam MM, Mufson EJ, Wainer BH, Levey AI (1983) Central cholinergic pathways in the rat: an overview based on an alternative nomenclature (Ch1-Ch6). Neuroscience 10, 4:1185-1201.

Mesulam M (1999) Neuroplasticity failure in Alzheimer's disease: bridging the gap between plaques and tangles. Neuron 24:521-529.

Mesulam M (2004) The cholinergic lesion of Alzheimer's disease: Pivotal factor or side show? Learn Mem 11:43-49.

Mikulecká A, Krsek P, Mares P (2000) Nonconvulsive kainic acid-induced seizures elicit age-dependent impairment of memory for the elevated plus-maze. Epilepsy Behav 1, 6:418-426.

Mikulecka A, Hlinak Z, Mares P (1999) Behavioural effects of a subconvulsive dose of kainic acid in rats. Behav Brain Res 101:21-28.

Milgram NW, Isen DA, Mandel D, Palantzas H, Pepkowski MJ (1988) Deficits in spontaneous behaviour and cognitive function following systemic administration of kainic acid. Neurotoxicology 9, 4:611-624.

Miracle AD, Brace MF, Huyck KD, Singler SA, Wellman CL (2006) Chronic stress impairs recall of extinction of conditioned fear. Neurobiol Learn Mem 85, 3:213-218. 
Moe KE, Vitiello ME, Larsen LH, Prinz PN (1995) Symposium: Cognitive processes and sleep disturbances: Sleep/wake patterns in Alzheimer's disease: relationship with cognition and function. J Sleep Res 4, 1:15-20.

Moga MM (1998) 192 IgG SAPorin abolishes $\mathrm{p} 75$ neurotrophin receptor immunoreactivity in rat SCN. Neuroreport 9, 14:3197-3200.

Mohajeri MH, Saini K, Schultz JG, Wollmer MA, Hock C, Nitsch RM (2002) Passive immunization against beta-amyloid peptide protects central nervous system (CNS) neurons from increased vulnerability associated with an Alzheimer's disease-causing mutation. J Bio Chem 277, 36:33012-33017.

Mohapel P, Leanza G, Kokaia M, Lindvall O (2005) Forebrain acetylcholine regulates adult hippocampal neurogenesis and learning. Neurobiol Aging 26:939-946.

Mohs RC, Doody RS, Morris JC, Ieni JR, Rogers SL, Perdomo CA, Pratt RD; "312" Study Group (2001) A 1-year, placebo-controlled preservation of function survival study of donepezil in $\mathrm{AD}$ patients. Neurology 57, 3:481-488.

Montaron MF, Drapeau E, Dupret D, Kitchener P, Aurousseau C, Le Moal M, Piazza PV, Abrous DN (2006) Lifelong corticosterone level deteremines age-related decline in neurogenesis and memory. Neurobiol Aging 27:645-654.

Moore-Ede MC, Sulzman FM, Fuller CA (1984) The Clocks That Time Us. Harvard University Press, Cambridge, Massachusetts and London, England.

Motooka Y, Kondoh T, Nomura T, Tamaki N, Tozaki H, Kanno T, Nishizaki T (2001) Selective cholinergic denervation inhibits expression of long-term potentiation in the adult but not infant rat hippocampus. Brain Res Dev Brain Res 129, 1:119-123.

Mora F, Segovia G, del Arco A. (2007) Aging, plasticity and environmental enrichment: Structural changes and neurotransmitter dynamics in several areas of the brain. Brain Res Rev 55:78-88.

Morley JE (2002) The SAMP8 mouse: a model of Alzheimer's disease? Biogerontology 3:57-60.

Morris RGM, Garrud P, Rawlins JNP, O'Keefe J (1982) Place navigation impaired in rats with hippocampal lesions. Nature 297:681-683.

Mudo G, Belluardo N, Mauro A, Fuxe K (2007) Acute intermittent nicotine treatment induces fibroblast growth factor- 2 in the subventricular zone of the adult rat brain and enhances neuronal precursor cell proliferation. Neuroscience 145:470-483. 
Muir JL, Everitt BJ, Robbins TW (1994) AMPA-induced excitotoxic lesions of the basal forebrain: a significant role for the cortical cholinergic system in attentional function. $J$ Neurosci 14, 4:2313-2326.

Munoz DG, Feldman H (2000) Causes of Alzheimer's disease. CMAJ 162, 1:65-72.

Murakami N, Takahashi K, Kawashima K (1984) Effect of light on the acetylcholine concentrations of the suprachiasmatic nucleus in the rat. Brain Res $311,2: 358-360$.

Murray CL, Fibiger HC (1985) Learning and memory deficits after lesions of the nucleus basalis magnocellularis: reversal by physostigmine. Neuroscience 14, 4:1025-1032.

Nabeshima T, Nitta A (1994) Memory impairment and neuronal dysfunction induced by beta-amyloid protein in rats. Tohoku J Exp Med 174, 3:241-249.

Nadel L, Moscovitch M (1997) Memory consolidation, retrograde amnesia and the hippocampal complex. Curr Op Neurobiol 7, 2:217-227.

Nakatomi H, Kuriu T, Okabe S, Yamamoto S, Hatano O, Kawahara N, Tamura A, Kirino T, Nakafuku M (2002) Regeneration of hippocampal pyramidal neurons after ischemic brain injury by recruitment of endogenous neural progenitors. Cell 110:429-441.

Nanri M, Yamamoto J, Miyake H, Watanabe H (1998) Protective effect of GTS-21, a novel nicotinic receptor agonist, on delayed neuronal death induced by ischemia in gerbils. Jap J Pharmacol 76, 1:23-29.

Näslund J, Haroutunian V, Mohs R, Davis KL, Davies P, Greengard P, Buxbaum JD (2000) Correlation between elevated levels of amyloid beta-peptide in the brain and cognitive decline. JAMA 283, 12:1571-1577.

Nedelmann M, Wilhelm-Schwenkmezger T, Alessandri B, Heimann A, Schneider F, Eicke BM, Dieterich M, Kempski O (2007) Cerebral embolic ischemia in rats:

Correlation of stroke severity and functional deficit as important outcome parameter. Brain Res 1130:188-196.

Nikolov R, Rami A, Krieglstein J (1993) Endothelin-1 exacerbates focal cerebral ischaemia without exerting neurotoxic action in vitro. Eur J Pharmacol 248:205-208.

Nilsson M, Perfilieva E, Johansson U, Orwar O, Eriksson PS (1999) Enriched environment increases neurogenesis in the adult rat dentate gyrus and improves spatial memory. J Neurobiol 39:569-578.

Nitsch RM, Slack BE, Wurtman RJ, Growdon JH (1992) Release of Alzheimer amyloid precursor derivatives stimulated by activation of muscarinic acetylcholine receptors.

Science 258, 5080:304-307. 
Nordberg A (2001) Nicotinic receptor abnormalities of Alzheimer's disease: therapeutic implications. Bio Psychiatry 49, 3:200-210.

Oddo S, Caccamo A, Kitazawa M, Tseng BP, LaFerla FM (2003) Amyloid deposition precedes tangle formation in a triple transgenic model of Alzheimer's disease. Neurobiol Aging 24, 8:1063-1070.

Oddo S, Caccamo A, Shepherd JD, Murphy MP, Golde TE, Kayed R, Metherate R, Mattson MP, Akbari Y, LaFerla FM (2003) Triple-transgenic model of Alzheimer's disease with plaques and tangles: intracellular Abeta and synaptic dysfunction. Neuron $39,3: 409-421$.

Ohayon MM, Lemoine P, Arnaurd-Briant V, Dreyfus M (2002) Prevalence and consequences of sleep disorders in a shift worker population. J Psychosomatic Res 53:577-583.

Ostbye T, Crosse E (1994) Net economic costs of dementia in Canada. CMAJ 151, 10:1457-1464.

Page KJ, Everitt BJ, Robbins TW, Marston HM, Wilkinson LS (1991) Dissociable effects on spatial maze and passive avoidance acquisition and retention following AMPA- and ibotenic acid-induced excitotoxic lesions of the basal forebrain in rats: differential dependence on cholinergic neuronal loss. Neuroscience 43:457-472.

Panegyres PK (1998) The effects of excitotoxicity on the expression of the amyloid precursor protein gene in the brain and its modulation by neuroprotective agents. J Neural Trans105, 4-5:463-478.

Pang KC, Nocera R, Secor AJ, Yoder RM (2001) GABAergic septohippocampal neurons are not necessary for spatial memory. Hippocampus 11, 6:814-827.

Pang KCH, Miller JP, McAulcy JD (2004) Circadian rhythms in SAMP8: a longitudinal study of the effects of age and experience. Neurobiol Aging 25:111-123.

Pappas BA, de la Torre JC, Davidson CM, Keyes MT, Fortin T (1996) Chronic reduction of cerebral blood flow in the adult rat: late-emerging CA1 cell loss and memory dysfunction. Brain Res 708:50-58.

Parent MB, Baxter MG (2004) Septohippocampal acetylcholine: involved in but not necessary for learning and memory? Learn Mem 11:9-20.

Pei Z, Pang SF, Cheung RTF (2002) Administration of melatonin after onset of ischemia reduces the volume of cerebral infarction in a rat middle cerebral artery occlusion stroke model. Stroke 34:770-775. 
Perry EK, Tomlinson BE, Blessed G, Perry RH, Cross AJ, Crow TT (1981) Noradrenergic and cholinergic systems in senile dementia of Alzheimer type. Lancet 2, 8238:149

Perry EK, Johnson M, Kerwin JM, Piggott MA, Court JA, Shaw PJ, Ince PG, Brown A, Perry RH (1992) Convergent cholinergic activities in aging and Alzheimer's disease. Neurobiol Aging 13:393-400.

Phillips RG, LeDoux JE (1992) Differential contribution of amygdala and hippocampus to cued and contextual fear conditioning. Behav Neurosci 106, 2:274-285.

Pittel Z, Heldman E, Barg J, Haring R, Fisher A (1996) Muscarinic control of amyloid precursor protein secretion in rat cerebral cortex and cerebellum. Brain Res 742, 1-2:299304.

Pizzo DP, Thal LJ, Winkler J (2002) Mnemonic deficits in animals depend upon the degree of cholinergic deficit and task complexity. Exp Neurol 177, 1:292-305.

Pollak CP, Perlick D (1991) Sleep problems and institutionalization of the elderly. J Ger Psychiatry Neurol 4, 4:204-210.

Ponnusamy R, Poulos AM, Fanselow MS (2007) Amygdala-dependent and amygdalaindependent pathways for contextual fear conditioning. Neuroscience 147, 4:919-927.

Porsolt RD, Anton G, Vlavet N, Jalfre M (1978) Behavioural despair in rats: a new model sensitive to antidepressant treatments. Eur J Pharmacol 47:379-391.

Qiu Y, Wu XJ, Chen HZ (2003) Simultaneous changes in secretory amyloid precursor protein and beta-amyloid peptide release from rat hippocampus by activation of muscarinic receptors. Neurosci Lett 352, 1:41-44.

Quirion R (1993) Cholinergic markers in Alzheimer disease and the autoregulation of acetylcholine release. J Psychiatry Neurosci 18, 5:226-234.

Radecki DT, Brown LM, Martinez J, Teyler TJ (2005) BDNF protects against stressinduced impairments in spatial learning and memory and LTP. Hippocampus 15:246-253.

Ralph MR, Foster RG, Davis FC, Menaker M (1990) Transplanted suprachiasmatic nucleus determines circadian period. Science 247:975-978.

Rasch JB, Gais S (2006) Sleep to remember. Neuroscientist 12, 5:410-424.

Reisberg B, Doody R, Stoffler A (2003) A randomized, placebo controlled study of memantine, an uncompetitive NMDA antagonists, in patients with moderate to severe Alzheimer's disease. New Eng J Med 348:1333-1341. 
Reiter RJ (1995) Oxidative processes and antioxidative defense mechanisms in the aging brain. FASEB 9:526-533.

Ridley RM, Samson NA, Baker HF, Johnson JA (1988) Visuospatial learning impairment following lesion of the cholinergic projection to the hippocampus. Brain Res 456, 1:7187.

Rissman RA, Lee KF, Vale W, Sawchenko PE (2007) Corticotropin-releasing factor receptors differentially regulate stress-induced tau phophorylation. J Neurosci 27 , 24:6552-6562.

Robertson RT, Gallardo KA, Clayton KJ, Ha DH, Ku K-H, Yu BP, Lauterborn JC, Wiley RG, Yu J, Gall CM, Leslie FM (1998) Neonatal treatment with 192 IgG SAPorin produces long-term forebrain cholinergic deficits and reduces branching and spine density of neocortical pyramidal neurons. Cereb Cortex 8:142-155.

Robinson SR, Bishop GM (2002) Abeta as a bioflocculant: implications for the amyloid hypothesis of Alzheimer's disease. Neurobiol Aging 23, 6:1051-1072.

Roe CM, Xiong C, Miller JP, Morris JC (2007) Education and Alzheimer disease without dementia: support for the cognitive reserve hypothesis. Neurology 68, 3:223-228.

Romanelli MF, Morris JC, Ashkin K, Coben LA (1990) Advanced Alzheimer's disease is a risk factor for late-onset seizures, Arch Neurol 47, 8:847-850.

Rossor MN, Iversen LL, Reynolds GP, Mountjoy CQ, Roth M (1984) Neurochemical characteristics of early and late onset types of Alzheimer's disease. Brit Med J 288, 6422:961-964.

Rouch I, Wild P, Ansiau D, Marquie JC (2005) Shiftwork experience, age and cognitive performance. Ergonomics 48, 10:1282-1293.

Rubio A, Pérez M, Avila J (2006) Acetylcholine receptors and tau phosphorylation. Curr Mol Med 6, 4:423-428.

Sakellaris PC, Peterson A, Goodwin A, Winget CM, Vernikos-Danellis J (1975) Response of mice to repeated photoperiod shifts: susceptibility to stress and barbiturates. Proc Soc Exp Biol Med 149, 3:677-680.

Sanders MJ, Wiltgen BJ, Fanselow MS (2003) The place of the hippocampus in fear conditioning. Eur J Pharmacol 463:217-223.

Sapolsky RM (1999) Glucocorticoids, stress and their adverse neurological effects: relevance to aging. Exp Gerontol 34:721-732. 
Sapolsky RM (2000) Glucocorticoids and hippocampal atrophy in neuropsychiatric disorders. Arch Gen Psychiatry 57:925-935.

Schäbitz WR, Berger C, Kollmar R, Seitz M, Tanay E, Kiessling M, Schwab S, Sommer $C$ (2004) Effect of brain-derived neurotrophic factor treatment and forced arm use on functional motor recovery after small cortical ischemia. Stroke 35, 4:992-997.

Scheinder JA, Arvanitakis Z, Bang W, Bennett DA (2007) Mixed brain pathologies account for most dementia cases in community-dwelling older persons. Neurology 1, 1:18.

Schliebs R (1998) Basal forebrain cholinergic dysfunction-experimental approzches and the diseased brain. Int J Dev Neurosci 16:601-603.

Schliebs R, Arendt T (2006) The significance of the cholinergic system in the brain during aging and Alzheimer's disease. J Neural Trans 113:1625-1644.

Schmidt-Kastner R, Freuend TF (1991) Selective vulnerability of the hippocampus in brain ischemia. Neuroscience 40, 3:599-636.

Schneider JA, Arvanitakis Z, Bang W, Bennett DA (2007) Mixed brain pathologies account for most dementia cases in community-dwelling older persons. Neurology 69, 24:2197-2204.

Scoville WB, Milner B (1957) Loss of recent memory after bilateral hippocampal lesions. J Neurol Neurosurg Psychiatry 20, 1:11-21

Sei H, Kiuchi T, Chang HY, Morita Y (1992) Effects of an eight-hour advance of the light-dark cycle on sleep-wake rhythm in the rat. Neurosci Lett 137:161-164

Selkoe DJ, Podlisny MB (2002) Deciphering the genetic basis of Alzheimer's disease. Ann Rev Genomics Hum Gen 3:67-99.

Sherren N, Pappas BA (2005) Selective acetylcholine and dopamine lesions in neonatal rats produce distinct patterns of cortical dendritic atrophy in adulthood. Neuroscience 136:445-456.

Shi J, Yang SH, Stubley L, Day AL, Simpkins JW (2000) Hypoperfusion induces overexpression of $\beta$-amyloid precursor protein $\mathrm{mRNA}$ in a focal ischemic rodent model. Brain Res 853:1-4.

Shin EJ, Chae JS, Jung ME, Bing G, Ko KH, Kim WK, Wie MB, Cheon MA, Nah SY, Kim HC (2007) Repeated intracerebroventricular infusion of nicotine prevents kainateinduced neurotoxicity by activating the alpha7 nicotinic acetylcholine receptor.

Epilep Res 73, 3:292-298. 
Shors TJ, Miesegaes G, Beylin A, Zhao M, Rydel T, Gould E (2001) Neurogenesis in the adult is involved in the formation of trace memories. Nature 410:372-376.

Shors TJ (2006) Stressful experience and learning across the lifespan. Ann Rev Psychol 57:55-85.

Silveira DC, Holmes GL, Schachter SC, Geula C, Schomer DL (2000) Increased susceptibility to generalized seizures after immunolesions of the basal forebrain cholinergic neurons in rats. Brain Res 878, 1-2:223-227.

Skorzewska A, Bidzinski A, Lehner M, Turzynskam D, Wislowska-Stanek A, Sobikewska A, Szyndler J, Maciejak P, Taracha E, Plaznik A (2006) The effects of acute and chronic administration of corticosterone on rat behaviour in two models of fear responses, plasma corticosterone concentration, and c-Fos expression in the brain structures. Pharmacol Biochem Behav 85:522-534.

Snowden DA, Greiner LH, Mortimer JA, Riley KP, Greiner PA, Markesbery WR (1997) Brain infarction and the clinical expression of Alzheimer's disease: The nun study. JAMA 277, 10:813-817.

Snyder JS, Hong NS, McDonald RJ, Wojtowicz JM (2005) A role for adult neurogenesis in spatial long-term memory. Neuroscience 130:843-852.

Song IU, Kim JS, Kim YI, Eah KY, Lee KS (2007) Clinical significance of silent cerebral infarctions in patients with Alzheimer's disease. Cog Behav Neurol 20:93-98.

Song L, Che W, Min-wei W, Murakami Y, Matsumoto K (2006) Impairment of the spatial learning and memory induced by learned helplessness and chronic mild stress. Pharmacol Biochem Behav 83:186-193.

Spanswick SC, Epp JR, Keith JR, Sutherland RJ (2007) Adrenalectomy-induced granule cell degeneration in the hippocampus causes spatial memory deficits that are not reversed by chronic treatment with corticosterone or Fluoxetine. Hippocampus 17:137-146.

Sperk G (1994) Kainic acid seizures in the rat. Prog Neurobiol 42:1-32.

Squire LR (2007) Memory and the hippocampus: a synthesis from findings with rats, monkeys, and humans. In Sources: Notable Selections in Psychology, 2nd edition. Ed. T.F. Pettijohn. Dushkin Publishing Group/Brown and Benchmark Publishing, Guilford, CT.pg. 29-34.

Stern Y (2006) Cognitive reserve and Alzheimer's disease. Alzeimer's Dis Assoc Dis 20, 2:112-117.

Stevens CF (1998) A million dollar question: Does LTP = memory? Neuron 20:1-2. 
Stone WS (1989) Sleep and aging in animals: Relationships with circadian rhythms and memory. Clin Ger Med 5:363-379.

Stone WS, Rudd RJ, Parsons MW, Gold PE (1997) Memory scores in middle aged rats predict deficits in memory, paradoxical sleep and blood glucose regulations in old age. Exp Aging Res 23:287-300.

Stubley-Weatherly L, Harding JW, Wright JW (1996) Effects of discrete kainic acidinduced hippocampal lesions on spatial and contextual learning and memory in rats. Brain Res 716, 1-2:29-38.

Sun X, He G, Qing H, Zhou W, Dobie F, Cai F, Staufenbiel M, Huang LE, Song W (2006) Hypoxia facilitates Alzheimer's disease pathogenesis by up-regulating BACE1 gene expression. PNAS 103, 49:18727-18732.

Sutherland RJ, Whishaw IQ, Kolb B (1983) A behavioural analysis of spatial localization following electrolytic, kainite- or colchicines-induced damage to the hippocampal formation in the rat. Behav Brain Res 7:133-153

Sutherland RJ, Leung LS, Weisend MP, Schlife J, McDonald RJ (1997) An evaluation of the effects of partial hippocampal kindling on place navigation in rats on the Morris water task. Psychobiology, 25:126-132.

Sutherland RJ (2005) Cognitive processes. In The Behaviour of the Laboratory Rat. Eds IQ Whishaw and B Kolb. Oxford University Press, pg. 422-435.

Suzuki M, Yamaguchi T, Ozawa Y, Iwai A, Yamamoto M (1995) Effect of YM796, a novel muscarinic agonist, on the impairment of passive avoidance response in senescence-accelerated mice. Pharmacol Biochem Behav 51, 4:623-626.

Suzuki M, Yamaguchi T, Ozawa Y, Ohyama M, Yamamoto M (1995b) Effects of (-)-S2,8-dimethyl-3-methylene-1-oxa-8-azaspiro[4,5]decane L-tartrate monohydrate (YM796), a novel muscarinic agonist, on disturbance of passive avoidance learning behaviour in drug-treated and senescence-accelerated mice. J Pharmacol Exp Ther 275, 2:728-736.

Swaab DF, Fliers E, Partiman TS (1981) The suprachiasmatic nucleus of the human brain in W.N. Tapp, F.A. Holloway, Phase shifting circadian rhythms produces retrograde amnesia. Science 211, 4486:1056-1058.

Takada Y, Yonezawa A, Kume T, Katsuki H, Kaneko S, Sugimoto H, Akaike A (2003) Nicotinic acetylcholine receptor-mediated neuroprotection by donepezil against glutamate neurotoxicity in rat cortical neurons. J Pharmacol Exp Ther 306, 2:772-777. 
Tanaka S, Liu L, Kimura J, Shiojiri S, Takahashi Y, Kitaguchi N, Nakamura S, Ueda K (1992) Age-related changes in the proportion of amyloid precursor protein mRNAs in Alzheimer's disease and other neurological disorders. Mol Brain Res 15:303-310.

Tapp WN, Holloway FA (1981) Phase shifting circadian rhythms produces retrograde amnesia. Science 211, 4486:1056-1058.

Tariot PN, Farlow MR, Grossberg GT, Graham SM, McDonald S, Gergel I (2004) Memantine treatment in patients with moderate to severe Alzheimer's disease already receiving donepezil: a randomized controlled trial. JAMA 291:317-324.

Tariq M, Khan HA, Elfaki I, Al Deeb S, Al Moutaery K (2005) Neuroprotective effect of nicotine against 3-nitropropionic acid (3-NP)-induced experimental Huntington's disease in rats. Brain Res Bull 67, 1-2:161-168.

Thompson PJ, Duncan JS (2005) Cognitive decline in severe intractable epilepsy. Epilepsia 46, 11:1780-1787.

Tiraboschi P, Hansen LA, Thal LJ, Corey-Bloom J (2004) The importance of neuritic plaques and tangles to the development and evolution of $\mathrm{AD}$. Neurology 62, 11:19841989.

Trofimiuk E, Braszko JJ (2008) Alleviation by Hypericum perforatum of the stressinduced impairment of spatial working memory in rats. Naunyn Schmiedebergs Arch Pharmacol 376, 6:463-471.

Tulving E, Markowitsch HJ (1998) Episodic and declarative memory: role of the hippocampus. Hippocampus 8:198-204.

Turek FW, Penev P, Zhang Y, van Reeth O, Zee P (1995) Effects of age on the circadian system. Neurosci Biobehav Rev 19, 1:53-58.

van Praag H, Kempermann G, Gage FH (1999) Running increases cell proliferation and neurogenesis in the adult mouse dentate gyrus. Nat Neurosci 2, 3:266-270.

van Praag H, Kemperman G, Gage FH (2000) Neural consequences of environmental enrichment. Nat Rev Neurosci 1:191-198.

van Someren EJW, Mirmiran M, Swaab DF (1993) Non-pharmacological treatment of sleep and wake disturbances in aging and Alzheimer's disease: chronobiological perspectives. Behav Brain Res 57:235-253.

van Someren EJW, Hagebeuk EEO, Lijzenga C, Scheltens P, de Rooij SEJA, Jonker C, Pot A, Mirmiran M, Swaab DF (1996) Circadian rest-activity rhythm disturbances in Alzheimer's disease. Biol Psychiatry 40:259-270. 
van Someren EJW (2000) Circadian and sleep disturbances in the elderly. Exp Gerontol $35: 1229-1237$.

Vargha-Khadem F, Gadian DG, Watkins KE, Connelly A, Van Paesschen W, Mishkin M (1997) Differential effects of early hippocampal pathology on episodic and semantic memory. Science 277:376-380.

Vaynman S, Ying Z, Gomez-Pinilla F (2004) Hippocampal BDNF mediates the efficacy of exercise on synaptic plasticity and cognition. Eur J Neurosci 20, 10:2580-2590.

Verbois SL, Hopkins DM, Scheff SW, Pauly JR (2003) Chronic intermittent nicotine administration attenuates traumatic brain injury-induced cognitive dysfunction. Neuroscience 119, 4:1199-1208.

Verdile G, Fuller S, Atwood CS, Laws SM, Gandy SE, Martins RN (2004) The role of beta amyloid in Alzheimer's disease: still a cause of everything or the only one who got caught? Pharmacol Res 50, 4:397-409.

Vermeer SE, Longstreth WT, Koudstaal PJ (2007) Silent brain infarcts: a systematic review. Lancet Neurol 6:611-619.

Vloeberghs E, Van Dam D, Engelborghs S, Nagels G, Staufenbiel M, De Deyn PP (2001) Altered circadian locomotor activity in APP23 mice: a model for BPSD disturbances, Eur J Neurosci 20, 10:2757-2766.

Vuckovich JA, Semel ME, Baxter MG (2004) Extensive lesions of cholinergic basal forebrain neurons do not impair spatial working memory. Learn Mem 11, 1:87-94.

Vyas A, Nitra R, Rao BSS, Chattarji S (2002) Chronic stress induces contrasting patterns of dendritic remodeling in hippocampal and amygdaloid neurons. J Neurosci 22, $15: 6810-6818$.

Waite JJ, Chen AD, Wardlow ML, Wiley RG, Lappi DA, Thal LJ (1995) 192 immunoglobulin G-saporin produces graded behavioural and biochemical changes accompanying the loss of cholinergic neurons of the basal forebrain and cerebellar Purkinje cells. Neuroscience 65, 2:463-476.

Waite JJ, Thal LJ (1996) Lesions of the cholinergic nuclei in the rat basal forebrain: excitotoxic vs. an immunotoxin. Life Sci 58, 22:1947-1953.

Waite JJ, Holschneider DP, Scremin OU (1999) Selective immuno-toxin induced cholinergic deafferentation alters blood flow distribution in the cerebral cortex. Brain Res 818:1-11.

Waite JJ, Wardlow ML, Power AE (1999) Deficit in selective and divided attention associated with cholinergic basal forebrain immunotoxic lesion produced by 192-saporin; 
motoric/sensory deficit associated with Purkinje cell immunotoxic lesion produced by OX7-saporin. Neurobiol Learn Mem 71, 3:325-352.

Walh F, Allix M, Plotkine M, Boulu RG (1992) Neurological and behavioural outcomes of focal ischemia in rats. Stroke 23:267-272.

Wanibuchi F, Nishida T, Yamashita H, Hidaka K, Koshiya K, Tsukamoto S, Usuda S (1994) Characterization of a novel muscarinic receptor agonist, YM796: comparison with cholinesterase inhibitors in in vivo pharmacological studies. Eur J Pharmacol 265, 3:151158.

Watanabe Y, Gould E, McEwen BS (1992) Stress induces atrophy of apical dendrites of hippocampal CA3 pyramidal neurons. Brain Res 588:341-345.

Wiley RG, Oeltmann TN, Lappi DA (1991) Immunolesioning: selective destruction of neurons using immunotoxin to rat NGF receptor. Brain Res 562:149-153.

Wilson RS, Arnold SE, Schneider JA, Kelly JF, Tang Y, Bennett DA (2006) Chronic psychological distress and risk of Alzheimer's disease in old age. Neuroepidemiology 27, 3:143-154.

Wiltgen BJ, Sanders MJ, Anagnostaras SG, Sage JR, Fanselow MS (2006) Context fear learning in the absence of the hippocampus. J Neurosci 26, 20:5484-5491.

Winblad B, Engedal K, Soininen H, Verhey F, Waldemar G, Wimo A, Wetterholm AL, Zhang R, Haglund A, Subbiah P, Donepezil Nordic Study Group (2001) A 1-year, randomized, placebo-controlled study of donepezil in patients with mild to moderate $\mathrm{AD}$. Neurology 57, 3:489-495.

Windle V, Szymanska A, Granter-Button S, White C, Buist R, Peeling J, Corbett D (2006) An analysis of four different methods of producing focal cerebral ischemia with endothelin-1 in the rat. Exp Neurol 201:324-334.

Witting W, Kwa IH, Eikelenboom P, Mirmiran M, Swaab DF (1999) Alterations in the circadian rest-activity rhythm in aging and Alzheimer's disease. Biol Psychiatry 27:564572.

Woolf NJ, Butcher LL (1982) Cholinergic projections to the basolateral amygdala: a combined Evan's blue and acetylcholinesterase analysis. Brain Res Bull 8:751-763.

Works SJ, Wilson RE, Wellman CL (2004) Age-dependent effect of cholinergic lesion on dendritic morphology in rat frontal cortex. Neurobiol Aging 25:963-974.

Wu YH, Feenstra MGP, Zhou JN, Liu RY, Torano JS, van Kan HJM, Fischer DF, Ravid $R$, Swaab DF (2003) Molecular changes underlying reduced pineal melatonin levels in 
Alzheimer's disease: Alterations in preclinical and clinical stages. J Clin Endocrinol Metab 88, 12:5898-5906.

Yamaguchi T, Suzuki M, Yamamoto M (1995) YM796, a novel muscarinic agonist, improves the impairment of learning behaviour in a rat model of chronic focal cerebral ischemia. Brain Res 669:107-114.

Yonemori F, Yamada H, Yamaguchi T, Uemura A, Tamura A (1996) Spatial memory disturbance after focal cerebral ischemia in rats. J Cereb Blood Flow Metab 16, 5:973980.

Yu J, Pizzo DP, Hutton LA, Perez-Polo JR (1995) Role of the cholinergic system in the regulation of neurotrophin synthesis. Brain Res 705, 1-2:247-252.

Zamani MR, Allen YS, Owen GP, Gray JA (1997) Nicotine modulates the neurotoxic effect of beta-amyloid protein(25-35)) in hippocampal cultures. Neuroreport 8, 2:513517.

Zatz M, Herkenham MA (1981) Intraventricular carbachol mimics the phase-shifting effect of light on the circadian rhythm of wheel-running activity. Brain Res 212, 1:234238.

Zenardi A, Ferrari R, Leo G, Maskos U, Changeux P, Zoli M (2007) Loss of high-affinity nicotinic recptors increases vulnerability to excitotoxic lesion and decreases positive effects of an enriched environment. FASEB 21:4028-4037.

Zhang Y, Zee PC, Kirby JD, Takahashi JS, Turek FW (1993) A cholinergic antagonist, mecamylamine, blocks light-induced Fos immunoreactivity in specific regions of the hamster suprachiasmatic nucleus. Brain Res 615:107-112.

Zhu XO, Waite PME (1998) Cholinergic depletion reduces plasticity of barrel field cortex. Cereb Cortex 8:63-72. 